Prepared in Cooperation with the Arkansas Natural Resources Commission and the Arkansas Geological Survey

\title{
Water Levels and Selected Water-Quality Conditions in the Mississippi River Valley Alluvial Aquifer in Eastern Arkansas, 2006
}

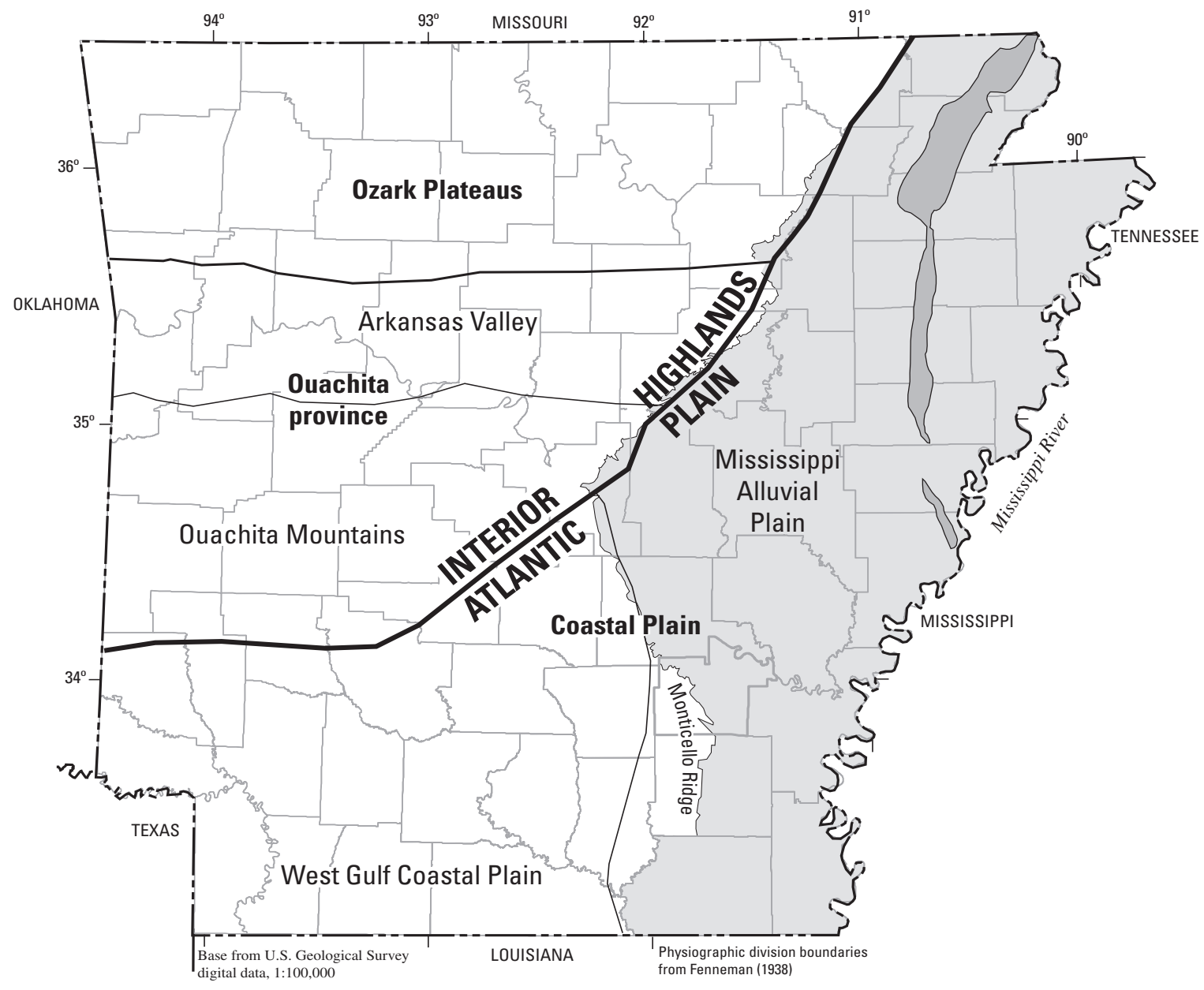

Scientific Investigations Report 2008-5092 


\section{Water Levels and Selected Water-Quality Conditions in the Mississippi River Valley Alluvial Aquifer in Eastern Arkansas, 2006}

By T.P. Schrader

In cooperation with the Arkansas Natural Resources Commission and the Arkansas Geological Survey

Scientific Investigations Report 2008-5092 


\section{U.S. Department of the Interior DIRK KEMPTHORNE, Secretary}

\section{U.S. Geological Survey \\ Mark D. Myers, Director}

\section{U.S. Geological Survey, Reston, Virginia: 2008}

For product and ordering information:

World Wide Web: http://www.usgs.gov/pubprod

Telephone: 1-888-ASK-USGS

For more information on the USGS--the Federal source for science about the Earth, its natural and living resources, natural hazards, and the environment:

World Wide Web: http://www.usgs.gov

Telephone: 1-888-ASK-USGS

Any use of trade, product, or firm names is for descriptive purposes only and does not imply endorsement by the U.S. Government.

Although this report is in the public domain, permission must be secured from the individual copyright owners to reproduce any copyrighted materials contained within this report.

Suggested citation:

Schrader, T.P., 2008, Water levels and selected water-quality conditions in the Mississippi River Valley alluvial aquifer in eastern Arkansas, 2006: U.S. Geological Survey Scientific Investigations Report 2008-5092, 73 p. 


\section{Contents}

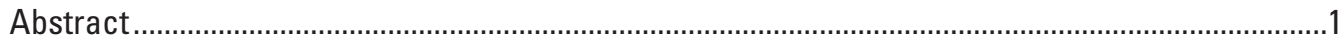

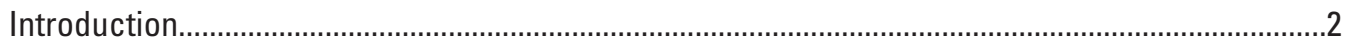

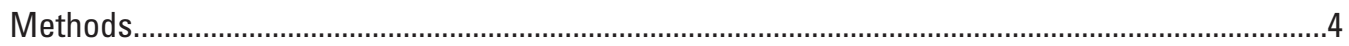

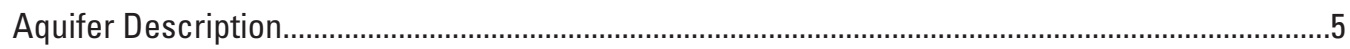

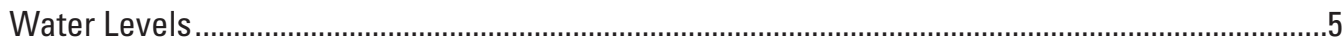

Potentiometric Surface ....................................................................................................

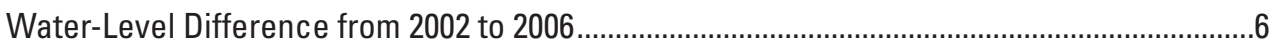

Long-Term Water-Level Changes.......................................................................................

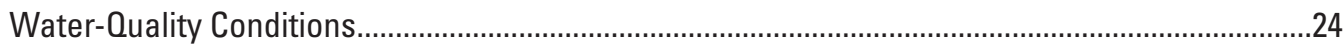

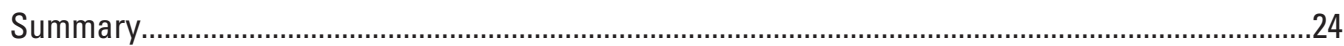

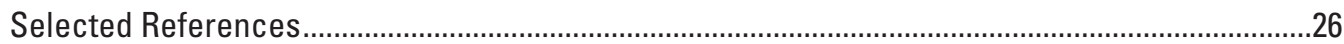

Appendix 1. Information pertaining to water levels measured in wells completed in the Mississippi River Valley alluvial aquifer in eastern Arkansas, spring 2006........................28

Appendix 2. Information pertaining to the difference in water levels measured in 2002 and 2006 in the Mississippi River Valley alluvial aquifer in eastern Arkansas ...........................50

Appendix 3. Specific conductance and temperature data collected from wells completed in the Mississippi River Valley alluvial aquifer in eastern Arkansas, summer 2006 ................70

\section{Plates}

[In pocket]

1. Map showing potentiometric surface of the Mississippi River Valley alluvial aquifer, spring 2006

2. Map showing difference in water level from 2002 to 2006 in the Mississippi River Valley alluvial aquifer in eastern Arkansas

\section{Figures}

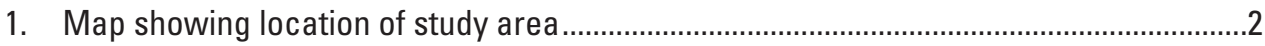

2. Graph showing estimated withdrawals from the Mississippi River Valley alluvial aquifer in Arkansas, 1965 to 2005........................................................................................

3. Diagram showing well-numbering system ................................................................

4. Water-level hydrographs (A to CC) for selected wells completed in the Mississippi River Valley alluvial aquifer ........................................................................................

5. Graph showing distribution of specific conductance in samples from wells completed in the Mississippi River Valley alluvial aquifer, 2006 .......................................................24

\section{Tables}

1. Range, mean, median, and correlation coefficient, $R^{2}$, of annual rise-decline in water level by county for wells in the Mississippi River Valley alluvial aquifer, 1982-2006........8 


\section{Conversion Factors and Datums}

\begin{tabular}{lcl}
\hline \multicolumn{1}{c}{ Multiply } & By & \multicolumn{1}{c}{ To obtain } \\
\hline inch (in.) & Length & \\
foot (ft) & 2.54 & centimeter $(\mathrm{cm})$ \\
mile (mi) & 0.3048 & meter $(\mathrm{m})$ \\
\hline & 1.609 & kilometer $(\mathrm{km})$ \\
\hline square mile $\left(\mathrm{mi}^{2}\right)$ & Area & \\
\hline & 2.590 & square kilometer $\left(\mathrm{km}^{2}\right)$ \\
\hline foot per year $(\mathrm{ft} / \mathrm{yr})$ & Flow rate & \\
cubic foot per second $\left(\mathrm{ft}^{3} / \mathrm{s}\right)$ & 0.3048 & meter per year $(\mathrm{m} / \mathrm{yr})$ \\
million gllons per day $(\mathrm{Mgal} / \mathrm{d})$ & 0.02832 & cubic meter per $\mathrm{second}\left(\mathrm{m}^{3} / \mathrm{s}\right)$ \\
\hline
\end{tabular}

Temperature in degrees Celsius $\left({ }^{\circ} \mathrm{C}\right)$ may be converted to degrees Fahrenheit $\left({ }^{\circ} \mathrm{F}\right)$ as follows: ${ }^{\circ} \mathrm{F}=\left(1.8 \mathrm{x}{ }^{\circ} \mathrm{C}\right)+32$

Temperature in degrees Fahrenheit $\left({ }^{\circ} \mathrm{F}\right)$ may be converted to degrees Celsius $\left({ }^{\circ} \mathrm{C}\right)$ as follows: ${ }^{\circ} \mathrm{C}=\left({ }^{\circ} \mathrm{F}-32\right) / 1.8$

Vertical coordinate information is referenced to the National Geodetic Vertical Datum of 1929 (NGVD of 1929).

Horizontal coordinate information is referenced to the North American Datum of 1983 (NAD of 1983).

Altitude, as used in this report, refers to distance above the vertical datum.

Specific conductance is given in microsiemens per centimeter at 25 degrees Celsius $\left(\mu \mathrm{S} / \mathrm{cm}\right.$ at $\left.25^{\circ} \mathrm{C}\right)$. 


\title{
Water Levels and Selected Water-Quality Conditions in the Mississippi River Valley Alluvial Aquifer in Eastern Arkansas, 2006
}

\author{
By T.P. Schrader
}

\section{Abstract}

During the spring of 2006, the U.S. Geological Survey, in cooperation with the Arkansas Natural Resource Commission and the Arkansas Geological Survey, measured water levels in 707 wells completed in the Mississippi River Valley alluvial aquifer in eastern Arkansas. Ground-water levels are affected by ground-water withdrawals resulting in depressions. In 2006, the lowest water-level altitude was 76 feet above the National Geodetic Vertical Datum of 1929 in the center of Arkansas County. The highest water-level altitude was 289 feet above the National Geodetic Vertical Datum of 1929 in northeastern Clay County on the west side of Crowleys Ridge. Two large depressions in the potentiometric surface are located in Arkansas, Lonoke, and Prairie Counties and west of Crowleys Ridge in Craighead, Cross, Lee, Monroe, Poinsett, St. Francis, and Woodruff Counties.

The elongated depression in Arkansas, Lonoke, and Prairie Counties has changed in areal extent or depth when compared to previous conditions of the aquifer. The area in Arkansas County at the southeastern half of the depression has not expanded horizontally during recent years, although the center of the depression has deepened. The area in Lonoke and Prairie Counties in the northwestern half of the depression has expanded horizontally in the deeper part of the depression. The 90-foot contour has expanded north and east in Lonoke County when compared with the 2004 potentiometric surface. Along the west side of Crowleys Ridge the 2006 potentiometric-surface map shows very little change in the area of this depression, although the deeper areas within the depression have expanded.

A map showing the difference in water level was constructed using 645 differences in water-levels measured in 633 wells during 2002 and 2006. The difference in measured water levels from 2002 to 2006 ranged from -24.0 feet to 25.0 feet, with a mean of -2.0 feet. The largest decline of -24.0 feet occurred in Poinsett County and the largest rise of 25.0 feet occurred in Randolph County. Out of the 645 differences, 481 were declines (74.6 percent), 12 were no difference (values of $0.0 \mathrm{ft}$ ) (1.8 percent), and 152 were rises (23.6 percent).
Long-term water-level trends were evaluated using hydrographs from 152 wells completed in the Mississippi River Valley alluvial aquifer for the period 1982 to 2006 . The mean annual rise or decline in water level for the entire study area was -0.32 feet per year with a range of -1.28 to 0.77 feet per year. Independence and White Counties are the only counties with a mean annual rise from 1982 to 2006. Mean annual declines between -0.50 feet per year and 0.00 feet per year occurred in Arkansas, Ashley, Chicot, Clay, Craighead, Crittenden, Drew, Jefferson, Lee, Mississippi, Monroe, Phillips, Poinsett, Prairie, Pulaski, Randolph, and Woodruff Counties. Mean annual declines between -1.00 feet per year and -0.50 feet per year occurred in Cross, Desha, Greene, Jackson, Lincoln, Lonoke, and St. Francis Counties.

The analysis of long-term water-level changes in Arkansas, Lonoke, and Prairie Counties shows the elongation of the depression in these three counties. Both Arkansas and Prairie Counties have two different rates of annual decline for the two hydrographs shown for each county. Water levels in the two wells near the Arkansas and White Rivers either have risen or declined at a slower rate than in the three wells in the center, northern, and western part of the depression. These rates of water-level change indicate that this depression is expanding in an elongated direction north and west into Lonoke and Prairie Counties. The depression west of Crowleys Ridge has five wells with hydrographs in or near the depression that can be used to characterize the rates of water-level change within the depression.

Water samples were collected from 65 wells completed in the Mississippi River Valley alluvial aquifer and measured onsite for specific conductance and temperature. Specific conductance ranged from 267 microsiemens per centimeter at 25 degrees Celsius at a well in Clay County to 2,960 microsiemens per centimeter at 25 degrees Celsius at a well in Chicot County. Four areas of relatively high specific conductance (greater than or equal to 1,000 microsiemens per centimeter at 25 degrees Celsius) occurred in Arkansas, Chicot, Craighead, and Prairie Counties. 


\section{Introduction}

The Mississippi Alluvial Plain (fig. 1) encompasses an area of approximately 32,000 square miles and includes parts of Arkansas and nearby States. Approximately 54 percent of the Mississippi Alluvial Plain covers the eastern one-third of Arkansas. The Mississippi River Valley alluvial aquifer (herein referred to as the alluvial aquifer) underlies the Mississippi Alluvial Plain in eastern Arkansas. Within Arkansas, the alluvial aquifer extends from the Missouri State line south to the Louisiana State line, and from the Mississippi River west to the Fall Line (the physiographic boundary between the Atlantic Plain and the Interior Highlands) and the Monticello
Ridge (a topographic feature in southeastern Arkansas), and near the western Ashley County line (fig. 1).

Agricultural land use in eastern Arkansas has increased since 1900 with production consisting predominately of rice, soybeans, cotton, and, in recent years, aquaculture, all of which are highly dependent on the availability of water. Eastern Arkansas receives sufficient precipitation to support these crops, receiving an average 46 to 54 inches of precipitation annually (Freiwald, 1984). However, during a critical portion of the growing season from late spring through early summer, most precipitation in eastern Arkansas falls as rain from widely scattered thunderstorms. Increasingly farmers are relying on ground water for agriculture and aquaculture.

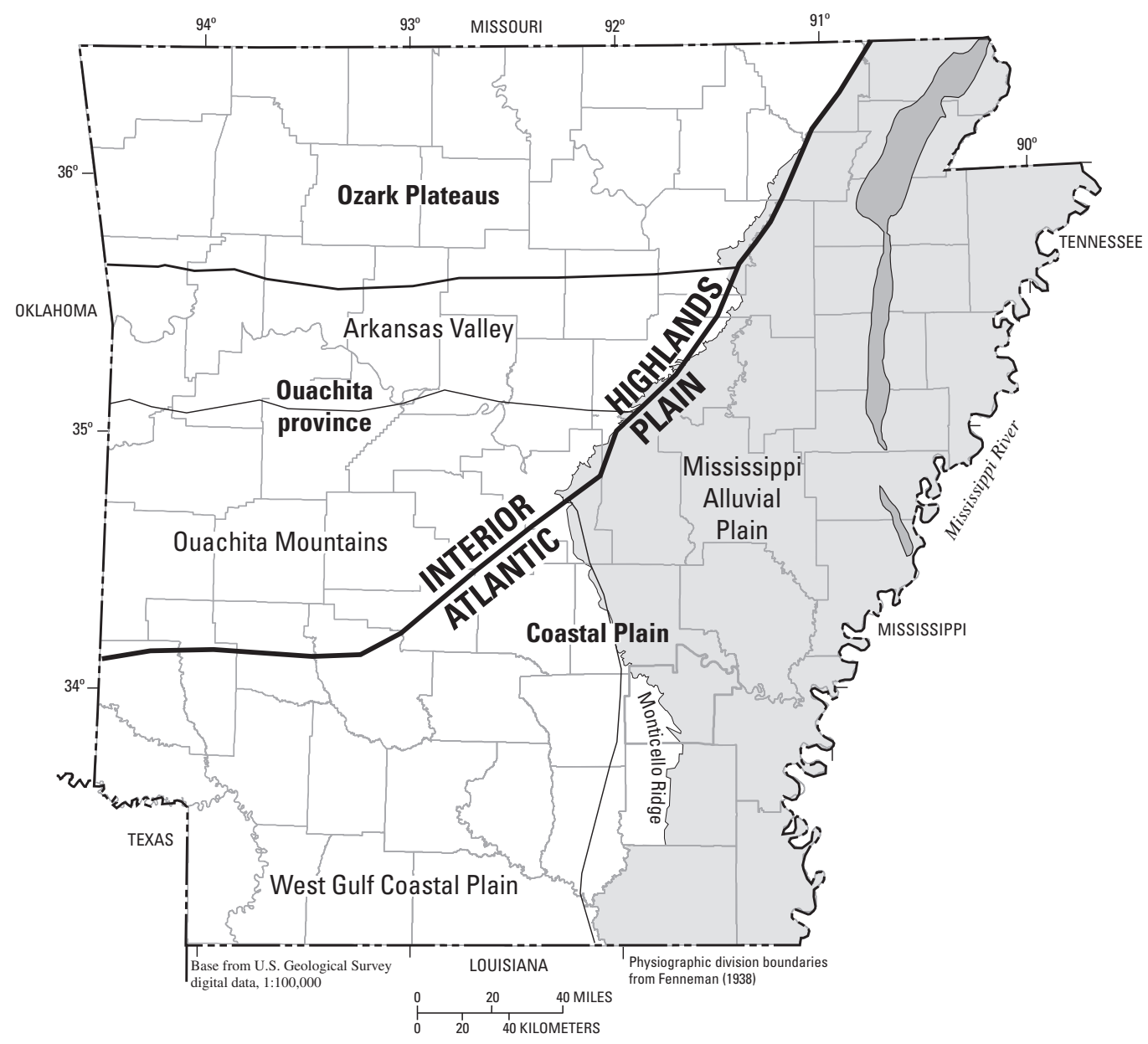

EXPLANATION

Study areas

Areal extent of Mississippi River Valley alluvial aquifer in eastern Arkansas

Crowleys Ridge
Physiographic division boundary

Major Division

- Province

Section

Figure 1. Location of study area. 
In 2005, estimated water withdrawals from the alluvial aquifer in Arkansas totaled about 7,252 million gallons per day (Mgal/d) (Terrance Holland, U.S. Geological Survey, written commun., 2007); estimated withdrawals were about 1,063 Mgal/d in 1965 (fig. 2) (Halberg and Stephens, 1966). Starting in 1970 and continuing in 5-year intervals through 2000, estimated water withdrawals from the alluvial aquifer in Arkansas totaled about 1,287 Mgal/d, 2,211 Mgal/d, 3,697 $\mathrm{Mgal} / \mathrm{d}, 3,534 \mathrm{Mgal} / \mathrm{d}, 4,360 \mathrm{Mgal} / \mathrm{d}, 5,056 \mathrm{Mgal} / \mathrm{d}$, and 6,585 Mgal/d (Halberg, 1972; Halberg, 1977; Holland and Ludwig, 1981; Holland, 1987; Holland, 1993; Holland, 1999; Holland, 2004). Since 1965 withdrawals from the alluvial aquifer have increased from about 1,063 Mgal/d to about 7,252 Mgal/d in 2005 , an increase of about 582 percent. Withdrawals have more than doubled in the last 20 years, about a 105 percent increase since 1985.

During the spring of 2006, the U.S. Geological Survey (USGS), in cooperation with the Arkansas Natural Resource Commission (ANRC) and the Arkansas Geological Survey, measured water levels in 707 wells completed in the alluvial aquifer in eastern Arkansas. The U.S. Department of Agriculture-Natural Resources Conservation Service (NRCS) measured water levels in 358 wells completed in the alluvial aquifer and provided these data to the ANRC. These data were made available to the USGS and were incorporated into the database used to develop a potentiometric-surface map of the alluvial aquifer for the spring of 2006. In the spring of 2006, a total of 720 water-level measurements ( 362 by the USGS and 358 by the NRCS) were collected from 707 wells. Because the USGS and NRCS both measure water levels in 13 wells, a total of 720 measurements were made in 707 wells. During the summer of 2006, water samples from 65 wells completed in the alluvial aquifer were measured for specific conductance and temperature. These measurements provided information for a database of selected water-quality data for the alluvial aquifer.

The purpose of this report is to describe the status and trends of water levels, specific conductance, and temperature in the alluvial aquifer. The report includes maps, long-term hydrographs, and data tables. Scheduled monitoring and evaluation of conditions in the alluvial aquifer provide information necessary for resource management.

The well-numbering system used in this report is based upon the locations of the wells according to the Federal land survey used in Arkansas. The component parts of a well number are the township number; the range number; the section number; three letters which indicate respectively, the quarter section, the quarter-quarter section, and the quarter-quarterquarter section in which the well is located; and a sequence number of the well in the quarter-quarter-quarter section. The letters are assigned counterclockwise, beginning with " $\mathrm{A}$ " in the northeast quarter or quarter-quarter or quarter-quarterquarter section in which the well is located. For example, well 01S03W04BBD16 (fig. 3) is located in Township 1 South,

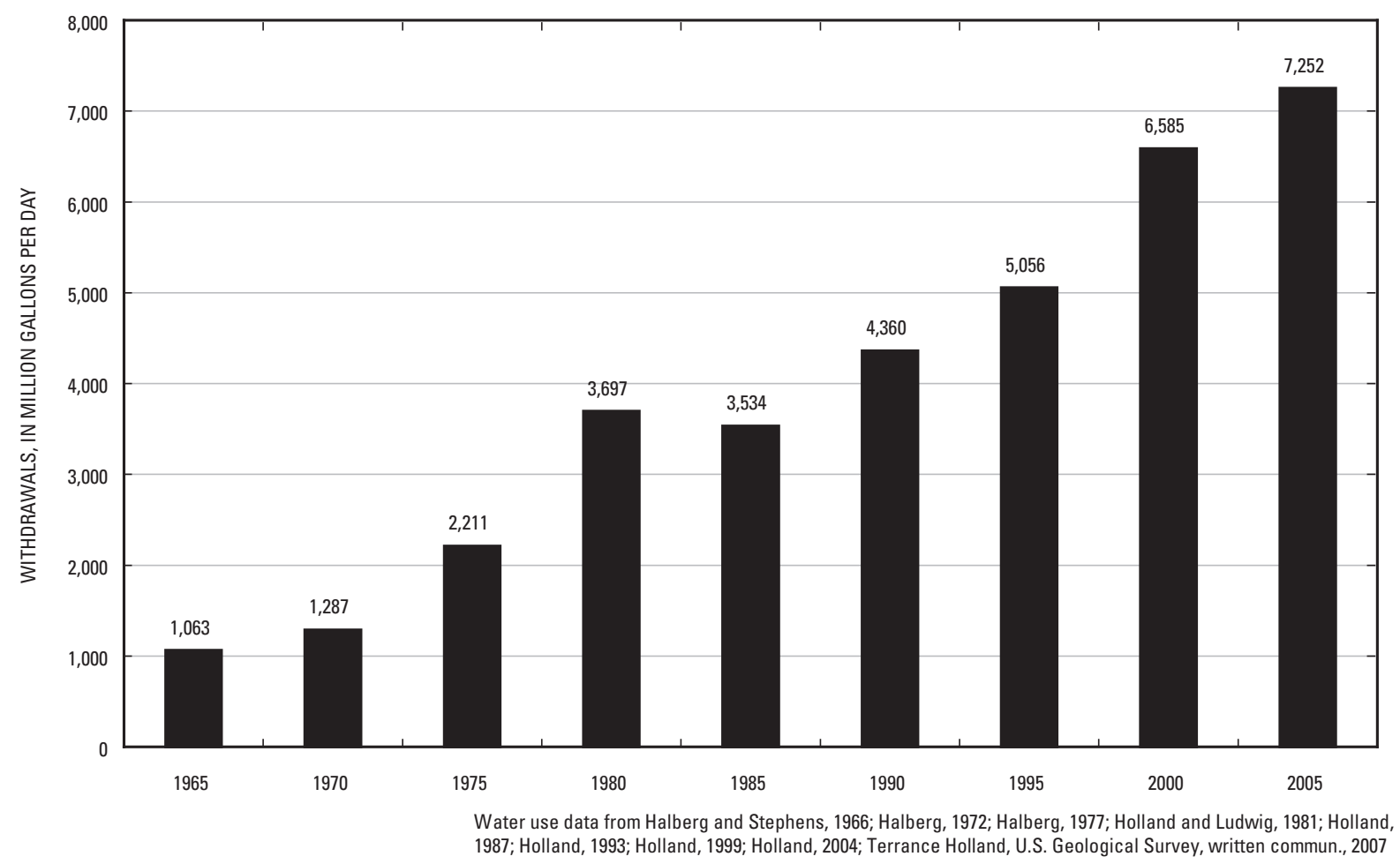

Figure 2. Estimated withdrawals from the Mississippi River Valley alluvial aquifer in Arkansas, 1965 to 2005. 


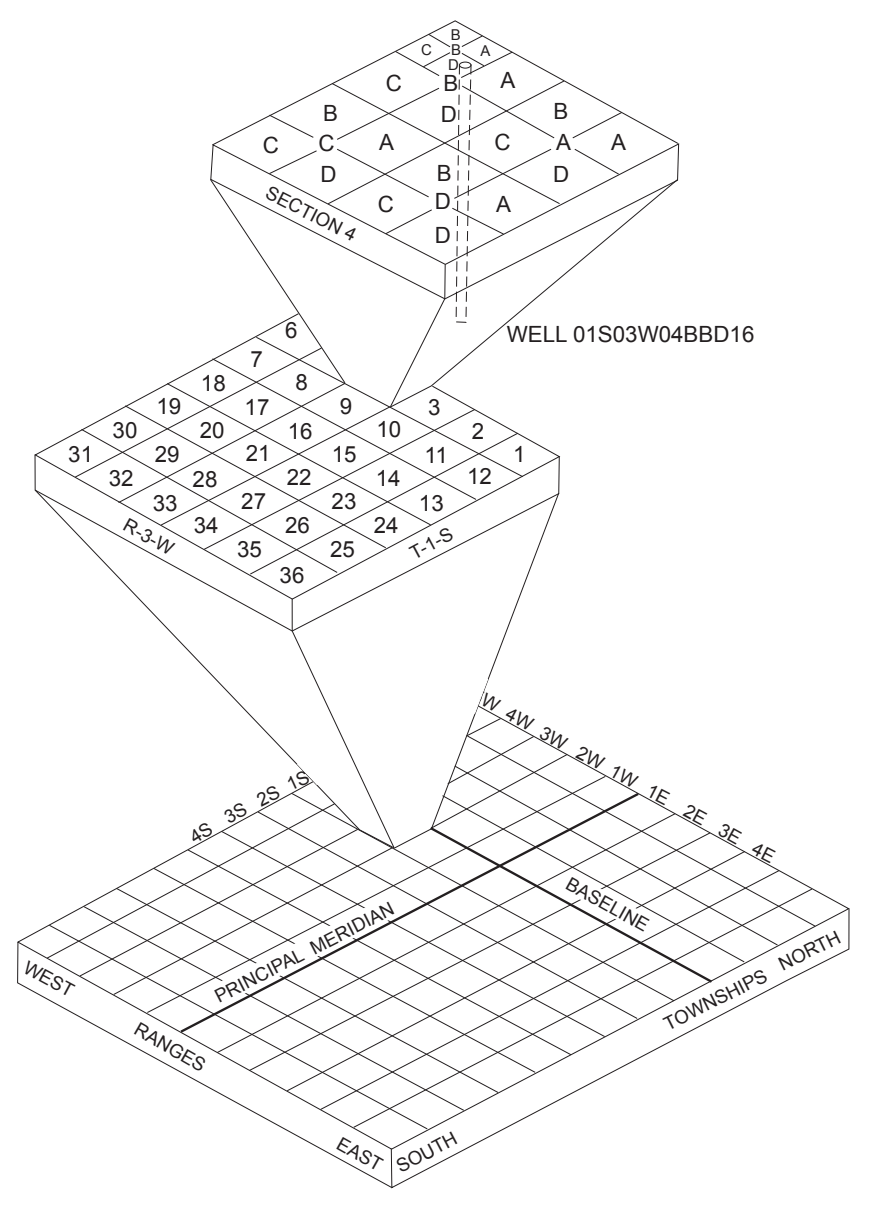

Figure 3. Well-numbering system.

Range 3 West, and in the southeast quarter of the northwest quarter of the northwest quarter of section 4 . This well is the 16 th well in this quarter-quarter-quarter section of section 4 from which data were collected.

\section{Methods}

Personnel from the USGS and the NRCS measured water levels from February 2006 to May 2006 from wells completed in the alluvial aquifer. Measurements by USGS personnel were made with steel or electric tapes graduated in hundredths of a foot, whereas measurements by NRCS personnel were in tenths of a foot or whole feet. The steel and electric tapes used by USGS personnel were calibrated during January 2006 prior to collecting measurements from wells. Calibration of steel and electric tapes was performed by comparing the field steel or electric tape to a standardized steel tape used only for calibration.
Well locations were measured using Global Positioning System receivers to acquire the horizontal coordinate information (latitude and longitude), based on the North American Datum of 1983. Land-surface altitude, feet above National Geodetic Vertical Datum of 1929 (NGVD of 1929), was determined for each well by superposition of the wells latitude and longitude on a topographic map and is accurate to about one-half the topographic contour interval of 5 or 10 feet (ft). Herein, all water-level and land-surface altitudes are referenced to NGVD of 1929.

One method for calculating the annual rise or decline is to take the difference between the final and initial water levels and divide by the period of time. This method is determined from two measurements and calculated values are dependent solely on the final and initial water levels. Linear regression is a second method that can be used with a hydrograph of water level in relation to time to calculate the annual rise or decline in water level. Linear regression is more robust because it includes all the measurements to determine the trend line, resulting in a value that is dependent on all water levels during the period of record. The slope, $\beta_{1}$, of the line is the annual rise or decline in water level. The intercept, $\beta_{0}$, would be the water level in the year 1900, the origin for the graph. This requires the assumption that the pumping rate was constant throughout the period of pumping. This condition is not commonly met or the data are not available to demonstrate that this has occurred. The predevelopment water level will not be discussed as this condition can not be demonstrated. The $\mathrm{R}^{2}$ term is the coefficient of determination, correlation coefficient, or the fraction of variance explained by regression. The $\mathrm{R}^{2}$ value gives the proportion of the total variability that can be accounted by the independent variable (Helsel and Hirsch, 1992). Values of $\mathrm{R}^{2}$ can range from 0.00 to 1.00 . A high value of $R^{2}$ can indicate a linear change in water level. A low value of $\mathrm{R}^{2}$ can indicate sporadic change in water level.

There are five assumptions associated with linear regression: (1) Y is linearly related to $\mathrm{X}$, (2) data used to fit the linear regression are representative of data of interest, (3) variance of the residuals is constant and does not depend on $\mathrm{X}$ or on anything else, (4) the residuals are independent, and (5) the residuals are normally distributed. The assumption of a normal distribution is involved only when testing hypotheses, requiring the residuals from the regression equation to be normally distributed (Helsel and Hirsch, 1992).

Specific conductance was measured in ground water from selected wells using specific-conductance meters with temperature compensation. Specific-conductance meters were calibrated twice daily by comparing the measurement of the specific conductance meter of two specific conductance calibration standards. Most of the wells sampled were irrigation wells sampled during pumping; specific conductance and temperature were monitored for 5 minutes or until the readings stabilized before values were recorded. For public supply and industrial wells, a minimum of three well volumes were purged and then specific conductance and temperature were 
monitored for 5 minutes or until the readings stabilized before values were recorded.

\section{Aquifer Description}

The alluvial aquifer comprises alluvial and terrace deposits of Quaternary age (Ackerman, 1996). Lithologically, the Quaternary alluvial and terrace deposits are similar, consisting of unconsolidated sediments that grade from gravel and coarse sand in the lower sections to silt and clay in the upper sections (Boswell and others, 1968). Because coarse sediments are contained in the lower sections of the alluvial and terrace deposits, the aquifer is capable of sustaining high yielding wells (Ackerman, 1996). Finer sediments in the upper sections of the alluvial and terrace deposits form a confining unit above much of the aquifer. This confining unit is thin or has been completely removed by erosion in some areas, especially near large rivers within the study area (Gonthier and Mahon, 1993). Channel fill, point bar, and backswamp deposits associated with present or former channels of large rivers have produced abrupt changes in lithology and result in large spatial variations in the hydraulic properties of the aquifer (Joseph, 1999).

Sedimentary rocks and unconsolidated sediments of Tertiary age or older underlie the alluvial aquifer and have been modified by geologic processes into an undulating surface (Mahon and Poynter, 1993). In most areas, these rocks and sediments are less permeable than the overlying alluvial and terrace deposits of Quaternary age and form the confining unit below the alluvial aquifer (Boswell and others, 1968).

In the northern half of the study area, the alluvial and terrace deposits of Quaternary age are separated by Crowleys Ridge (fig. 1), an erosional remnant of Tertiary-age deposits trending north-south from the Missouri-Arkansas border to northeastern Phillips County. Crowleys Ridge is a prominent topographic feature on the otherwise low-relief surface of the Mississippi Alluvial Plain and forms a physical barrier to ground-water flow in the alluvial aquifer.

\section{Water Levels}

Water-level measurements collected in wells completed in the alluvial aquifer (appendix 1) were used to produce a regional potentiometric-surface map (plate 1). A difference in water-level map from 2002 to 2006 (plate 2), was produced by subtracting water-level measurements in 2006 from measurements in 2002. Data from wells that have water-level measurements with a minimum 25-year period of record were used to produce hydrographs shown on figure 4 . The water-level changes shown in the hydrographs indicate long-term changes in hydrologic conditions. Long-term water-level changes shown by the hydrographs reflect the development of the cones of depression in the potentiometric surface.

\section{Potentiometric Surface}

The potentiometric-surface map (plate 1) shows the altitude at which water would have stood in tightly cased wells completed in the alluvial aquifer. The map on plate 1 is based on 720 water-level measurements ( 362 by USGS and 358 by NRCS) made in 707 wells during the spring of 2006 (appendix 1). The USGS and NRCS both measure water levels from the same 13 wells. The surface was mapped using the altitude of the water levels measured in the wells and is represented on the map by contours that connect points of equal altitude. The general direction of ground-water flow is perpendicular to the contours in the direction of decreasing potentiometric-surface altitude.

Ground-water levels are affected by ground-water withdrawals within the study area, often resulting in depressions. The depressions or other areas of reduced water level are shaded on plate 1. In 2006, the lowest water-level altitude was $76 \mathrm{ft}$ in the center of Arkansas County. The highest water-level altitude was $289 \mathrm{ft}$ in northeastern Clay County on the west side of Crowleys Ridge.

Previous reports described three large depressions in the alluvial aquifer potentiometric surface (Stanton and others, 1998; Joseph, 1999; Schrader, 2001; Reed, 2004; Schrader, 2006). A large, elongated area of depression extended across Arkansas, Lonoke, and Prairie Counties. Two shallower depressions were documented in Lee, Monroe, St. Francis, and Woodruff Counties, and in Craighead, Cross, and Poinsett Counties.

The elongated depression in Arkansas, Lonoke, and Prairie Counties has two areas that have changed in horizontal area or depth when compared to previous conditions of the aquifer. The area in Arkansas County at the southeastern half of the depression has not expanded horizontally from recent years, although the center of the depression has deepened. The Arkansas and White Rivers that bound Arkansas County on the southwestern and eastern county lines are hydrologically connected and provide recharge to the alluvial aquifer. A comparison of measured water-level altitudes from 1998 to 2006 at the lowest measurement point in the depression in central Arkansas County indicates that water levels rose from 1998 to 2002. The lowest measured water-level altitudes in the alluvial aquifer in Arkansas County in 1998, 2000, and 2002 were 78, 86, and 80 ft, respectively (Joseph, 1999; Schrader, 2001; Reed, 2004). The lowest measured water-level altitude in 2004 showed a decline of $10 \mathrm{ft}$ to $76 \mathrm{ft}$ since 2002 (Schrader, 2006). In 2006, the lowest water-level altitude was $76 \mathrm{ft}$, the same as in 2004 .

The area in Lonoke and Prairie Counties in the northwestern half of the depression has expanded horizontally in the deeper part of the depression. The 90-foot contour has expanded north and east in Lonoke County, when compared to the 2004 potentiometric surface (Schrader, 2006). In 2000, this depression (within the 90-foot contour) was elongated from southwest to northeast from Lonoke County into Prairie County. Between 2000 and 2004, the area decreased to a small 
oval near the center of Lonoke County. The lowest measured water-level altitudes in Lonoke County show little change in 2002, 2004, and 2006 with altitudes of $88 \mathrm{ft}, 89 \mathrm{ft}$, and $90 \mathrm{ft}$, respectively (Reed, 2004; Schrader, 2006).

Along the west side of Crowleys Ridge, two previously documented areas of depression expanded and coalesced into a single depression by 2002 (Reed, 2004). The 2006 potentiometric-surface map shows little change in the area of this depression, although the deeper areas within the depression have expanded. The 2006 potentiometric-surface map shows that the area enclosed by the 150 -foot contour is similar in area to the 2004 potentiometric-surface map. The two areas enclosed by 140 -foot contours have changed when compared with the 2004 potentiometric-surface map. The area in Lee, Monroe, St. Francis, and Woodruff Counties is similar in area to the 2004 potentiometric-surface map. The area in Cross and Poinsett Counties in 2004 has expanded north into Craighead County and east to intersect with Crowleys Ridge in the 2006 potentiometric-surface map. The 130-foot contour is shown in southwestern Poinsett, southwestern St. Francis, eastern Monroe, and northwestern and southwestern Lee Counties. The 130-foot contours in southwestern Poinsett, eastern Monroe, and southwestern Lee Counties have not been shown in previous potentiometric-surface maps. The 130 -foot contour in southwestern St. Francis and northwestern Lee Counties expanded in 2006. The lowest water-level measurement in the depression in Lee, Monroe, St. Francis, and Woodruff Counties has declined to $123 \mathrm{ft}$ (from $125 \mathrm{ft}$ in 2004) in northwestern Lee County.

In central Drew County, near the western boundary of the study area, a cone of depression was first noted in the 2002 potentiometric-surface map (Reed, 2004). The area enclosed by the 130-foot contour expanded in 2004 and the water level in the center of the cone declined by $8 \mathrm{ft}$ to $118 \mathrm{ft}$ (Schrader, 2006). In 2006, the area has decreased to approximately the same area as in the 2002 potentiometric-surface map.

Three areas of reduced water level were noted by Schrader (2001) in southeastern Arkansas-one in eastern Lincoln County, a second that extends from southern Desha County into northern Chicot County, and a third that extends from western Chicot County into eastern Ashley County. The area of reduced water level in southern Desha and northern Chicot Counties was first evident in the 1998 potentiometric surface (Joseph, 1999) and had expanded horizontally and vertically by 2000 (Schrader, 2001). This area expanded southward by 2002 but had not appreciably deepened. The potentiometric surface in this area showed very little change between 2002 and 2004 (Schrader, 2006). In the 2006 potentiometric surface, this area has expanded further north into Desha County and south into Chicot County. The expansion may be partially explained by better definition from additional data extending south into Chicot County. The areas in eastern Lincoln County and in western Chicot and eastern Ashley Counties were not evident in 1996 and 1998. The area in eastern Lincoln County expanded into northwestern Desha County and continued to deepen through 2004, with an altitude of
$118 \mathrm{ft}$ (Schrader, 2006) at its deepest point. In 2006, this area has expanded westward in Lincoln County, with a measured altitude of $119 \mathrm{ft}$ at its deepest point. The area in western Chicot and eastern Ashley Counties showed very little change in the 2004 potentiometric surface from the 2002 potentiometric surface (Schrader, 2006). In 2006, the depth of this area has not increased and is approximately the same as in the 2004 potentiometric surface.

A potentiometric area of reduced water level in Greene County noted in 1998 by Joseph (1999), and in 2000 by Schrader (2001), deepened by 2002 (Reed, 2004). This area contracted in 2004 (Schrader, 2006). In 2006, this area has expanded and deepened since 2004.

Six depressions are shown in the 2006 potentiometricsurface map that are not shown in previous alluvial aquifer potentiometric surfaces. A depression at the Prairie and White County line is enclosed by a 130-foot contour. In the 2004 potentiometric surface, the 130 -foot contour line shows a small lobe extending northeast from the larger depression (Schrader, 2006). In the 2006 potentiometric surface, the 130foot contour line is approximately oval shaped and separate from the larger depression. A second depression at the Craighead and Mississippi County line is enclosed by a 220 -foot contour. Four small depressions are located in northern Desha, southeastern Jefferson, northern Lee, and northeastern St. Francis Counties. Continued monitoring of the potentiometric surface will determine if these depressions are the result of short-term variations or long-term changes in the hydrologic conditions in the alluvial aquifer.

The regional direction of ground-water flow is generally to the south and east except where flow is affected by groundwater withdrawals; however, the flow direction is affected over substantial areas by depressions. Ground-water flow is indicated by the black arrows shown on plate 1 . West of Crowleys Ridge, depressions in Arkansas, Lonoke, and Prairie Counties capture ground-water flow from all directions. The flow along large sections of the Arkansas, Mississippi, and White Rivers is away from the rivers. East of Crowleys Ridge water flows from north to south along Crowleys Ridge and northeast to southwest along the Mississippi River. South of the Arkansas River the flow is towards the southeast, except in northwestern Desha County where flow is towards the area of reduced water level.

\section{Water-Level Difference from 2002 to 2006}

A map showing the difference in water level (plate 2) was constructed using 645 differences in water levels measured in 633 wells during 2006 (appendix 2) and 2002 (Reed, 2004). Differences in water level were calculated by subtracting the 2006 depth-to-water measurement from the 2002 depth-to-water measurement. Positive values indicate a rise and negative values indicate a decline in water level. Rises in water level are indicated on plate 2 with blue triangles pointing upward; declines in water level are indicated with red triangles point- 
ing downward. The triangles are scaled to the value of rise or decline.

The difference in measured water levels from 2002 to 2006 ranged from $-24.0 \mathrm{ft}$ to $25.0 \mathrm{ft}$, with a mean of $-2.0 \mathrm{ft}$. The largest decline of $-24.0 \mathrm{ft}$ occurred in Poinsett County and the largest rise of $25.0 \mathrm{ft}$ occurred in Randolph County. Out of the 645 differences on plate 2, 481 were declines (74.6 percent), 12 were no difference (values of $0.0 \mathrm{ft}$ ) (1.8 percent), and 152 were rises ( 23.6 percent). The three areas that have the greatest density of declines are west of Crowleys Ridge, eastern Craighead and Mississippi Counties, and in eastern Lonoke and Prairie Counties.

Five areas are dominated by rises in measured water levels. The largest area is in western and southern Arkansas, southeastern Jefferson, and northern Desha Counties adjacent to the Arkansas River. Another area is in eastern Cross and northern Crittenden Counties. Three small areas with waterlevel rises are located in western Lonoke County, southeastern White County, and adjacent to the eastern boundary of Crowleys Ridge in Craighead and Greene Counties.

\section{Long-Term Water-Level Changes}

Long-term water-level changes were evaluated using hydrographs from 152 wells in the alluvial aquifer for the period 1982 to 2006. Linear regression was used to calculate the trend in water-level change for each well for this period. The slope of the trend line represents the mean annual rise or decline in water level during the 25 -year period. Negative values denote a decline in water level. The minimum 25-year period is used to show long-term trends not dominated by short-term variations in climate and localized pumping rates on water levels in a single well. The hydrographs were grouped by county. Table 1 shows the number of wells, the range of values for the annual rise or decline in water level, the mean, and the median for each county. The mean annual rise or decline in water level for the entire study area was -0.32 feet per year ( $\mathrm{ft} / \mathrm{yr}$ ) with a range of -1.28 to $0.77 \mathrm{ft} / \mathrm{yr}$. Selected hydrographs are shown in figure 4 (wells A-CC, plate $1)$.

Long-term water-level changes vary substantially across the study area. Independence and White Counties are the only counties with a mean annual rise from 1982 to 2006. The rise in Independence County is determined from the data of one well. Mean annual declines between $-0.50 \mathrm{ft} / \mathrm{yr}$ and $0.00 \mathrm{ft} / \mathrm{yr}$ occurred in Arkansas, Ashley, Chicot, Clay, Craighead, Crittenden, Drew, Jefferson, Lee, Mississippi, Monroe, Phillips, Poinsett, Prairie, Pulaski, Randolph, and Woodruff Counties. Mean annual declines between $-1.00 \mathrm{ft} / \mathrm{yr}$ and $-0.50 \mathrm{ft} / \mathrm{yr}$ occurred in Cross, Desha, Greene, Jackson, Lincoln, Lonoke, and St. Francis Counties.

The analysis of long-term water-level changes (19822006) in Arkansas, Lonoke, and Prairie Counties shows the elongation of the depression in these three counties. Arkansas and Prairie Counties have two different rates of annual decline for the two hydrographs shown for each county. In Arkansas County, well 04S03W32BCB1 (fig. 4A) shows an annual water-level decline of about $0.80 \mathrm{ft} / \mathrm{yr}$ since 1982. Well A is located near the center of the depression in Arkansas County and generally shows a water-level decline during the 70-year period of record. Well 07S04W01DDD1 (fig. 4B) has an annual water-level rise of about $0.11 \mathrm{ft} / \mathrm{yr}$ since 1982. Well B is located near the Arkansas River and shows a relatively stable water level for the 79-year period. The water level in the Arkansas River is maintained by a lock and dam system and can be a source of water for the alluvial aquifer in southern and western Arkansas County. In Prairie County, well 02N04W32CCB1 (fig. 4X) is near the depression and the White River. This well has an annual water-level decline of about $0.29 \mathrm{ft} / \mathrm{yr}$ since 1982. Well 04N05W07CDC1 (fig. $4 \mathrm{~W}$ ) is located in the northern part of Prairie County and has an annual decline of about $0.66 \mathrm{ft} / \mathrm{yr}$ since 1982 . These two hydrographs show that the rate of decline in the northern part of the depression is more than two times greater than the rate of decline near the White River. Well 02S07W10CCB1 (fig. 4Q), near the western edge of the depression in Lonoke County, shows an annual water-level decline of about 0.88 $\mathrm{ft} / \mathrm{yr}$ since 1982 and shows a nearly continuous water-level decline during the 49 years of record. In Arkansas, Lonoke, and Prairie Counties, water levels in the two wells near the Arkansas and White Rivers either have risen or declined at a slower rate than in the three wells in the center, northern, and western part of the depression. These rates of waterlevel change indicate that this depression is expanding in an elongated direction north and west into Lonoke and Prairie Counties.

Water-level declines in neighboring counties are further evidence of the expansion of the depression centered in Arkansas, Lonoke, and Prairie Counties. In Jefferson County, well 03S08W24BBC1 (fig. 4N) has an annual water-level decline of about $0.69 \mathrm{ft} / \mathrm{yr}$ during the period 1982 to 2006. In Monroe County, well 03N01W20ABA1 (fig. 4S) has an annual waterlevel decline of about $0.66 \mathrm{ft} / \mathrm{yr}$ during the period 1982 to 2006. Long-term declines in these outlying wells indicate that the depression is expanding.

The depression west of Crowleys Ridge has five wells with hydrographs in or near the depression that can be used to characterize the rates of water-level change within the depression. Wells 07N01E05CDA1 (fig. 4H) in Cross County, 11N02E26AAB1 (fig. 4U) in Poinsett County, and 04N01W28CDD1 (fig. 4AA) in St. Francis County are in the depression and have annual declines of about $1.02 \mathrm{ft} / \mathrm{yr}, 1.28$ $\mathrm{ft} / \mathrm{yr}$, and $0.62 \mathrm{ft} / \mathrm{yr}$, respectively, since 1982. For the period of record, well $\mathrm{H}$ in Cross County, since 1946, and well U in Poinsett County, since 1958, have the largest declines in the alluvial aquifer, about $48.8 \mathrm{ft}$ and $56.1 \mathrm{ft}$, respectively. Wells 14N02E18BDD1 (fig. 4F) in Craighead County and 02N01E23BAA2 (fig. 4O) in Lee County near the outskirts of the depression have annual water-level declines of about 0.99 $\mathrm{ft} / \mathrm{yr}$ and $0.55 \mathrm{ft} / \mathrm{yr}$, respectively, since 1982. 
Table 1. Range, mean, median, and correlation coefficient, $R^{2}$, of annual rise-decline in water level by county for wells in the Mississippi River Valley alluvial aquifer, 1982-2006.

[Annual rise or decline in water level for each well is calculated using linear regression; negative value indicates decline; positive value indicates rise]

\begin{tabular}{|c|c|c|c|c|c|}
\hline County & $\begin{array}{l}\text { Number } \\
\text { of wells }\end{array}$ & $\begin{array}{c}\text { Range of } \\
\text { annual rise- } \\
\text { decline in } \\
\text { water level } \\
\text { (feet/year) }\end{array}$ & $\begin{array}{l}\text { Mean annual } \\
\text { rise-decline in } \\
\text { water level } \\
\text { (feet/year) }\end{array}$ & $\begin{array}{c}\text { Median annual } \\
\text { rise-decline in } \\
\text { water level } \\
\text { (feet/year) }\end{array}$ & $\begin{array}{l}\text { Range of } R^{2} \\
\text { values } \\
\text { for trend } \\
\text { line }\end{array}$ \\
\hline Arkansas & 27 & -0.80 to 0.77 & -0.12 & -0.15 & 0.00 to 0.80 \\
\hline Ashley & 9 & -0.33 to 0.11 & -0.14 & -0.18 & 0.03 to 0.72 \\
\hline Chicot & 3 & -0.40 to -0.11 & -0.26 & -0.26 & 0.19 to 0.71 \\
\hline Clay & 7 & -0.55 to -0.01 & -0.21 & -0.15 & 0.01 to 0.93 \\
\hline Craighead & 5 & -0.99 to 0.18 & -0.37 & -0.18 & 0.10 to 0.94 \\
\hline Crittenden & 6 & -0.62 to -0.15 & -0.37 & -0.35 & 0.24 to 0.93 \\
\hline Cross & 5 & -1.24 to -0.33 & -0.94 & -1.02 & 0.75 to 0.97 \\
\hline Desha & 5 & -0.80 to -0.07 & -0.50 & -0.62 & 0.19 to 0.87 \\
\hline Drew & 4 & -0.29 to -0.15 & -0.24 & -0.26 & 0.53 to 0.94 \\
\hline Greene & 5 & -0.73 to -0.11 & -0.53 & -0.66 & 0.13 to 0.85 \\
\hline Independence & 1 & 0.07 & 0.07 & 0.07 & 0.01 \\
\hline Jackson & 5 & -0.84 to -0.29 & -0.68 & -0.77 & 0.66 to 0.98 \\
\hline Jefferson & 6 & -0.69 to -0.18 & -0.32 & -0.24 & 0.33 to 0.92 \\
\hline Lee & 5 & -0.58 to -0.26 & -0.49 & -0.55 & 0.26 to 0.92 \\
\hline Lincoln & 3 & -0.84 to -0.33 & -0.57 & -0.55 & 0.67 to 0.85 \\
\hline Lonoke & 5 & -1.06 to -0.51 & -0.80 & -0.88 & 0.34 to 0.98 \\
\hline Mississippi & 8 & -0.22 to 0.00 & -0.09 & -0.09 & 0.00 to 0.47 \\
\hline Monroe & 8 & -0.55 to -0.01 & -0.28 & -0.27 & 0.00 to 0.90 \\
\hline Phillips & 3 & -0.29 to -0.07 & -0.18 & -0.18 & 0.07 to 0.71 \\
\hline Poinsett & 5 & -1.28 to 0.03 & -0.37 & -0.18 & 0.02 to 0.96 \\
\hline Prairie & 10 & -0.66 to 0.00 & -0.31 & -0.29 & 0.00 to 0.89 \\
\hline Pulaski & 1 & -0.33 & -0.33 & -0.33 & 0.58 \\
\hline Randolph & 1 & -0.26 & -0.26 & -0.26 & 0.74 \\
\hline St. Francis & 7 & -0.95 to -0.04 & -0.56 & -0.62 & 0.13 to 0.98 \\
\hline White & 3 & 0.11 to 0.29 & 0.19 & 0.18 & 0.20 to 0.62 \\
\hline Woodruff & 5 & -0.58 to 0.00 & -0.19 & -0.11 & 0.00 to 0.87 \\
\hline
\end{tabular}



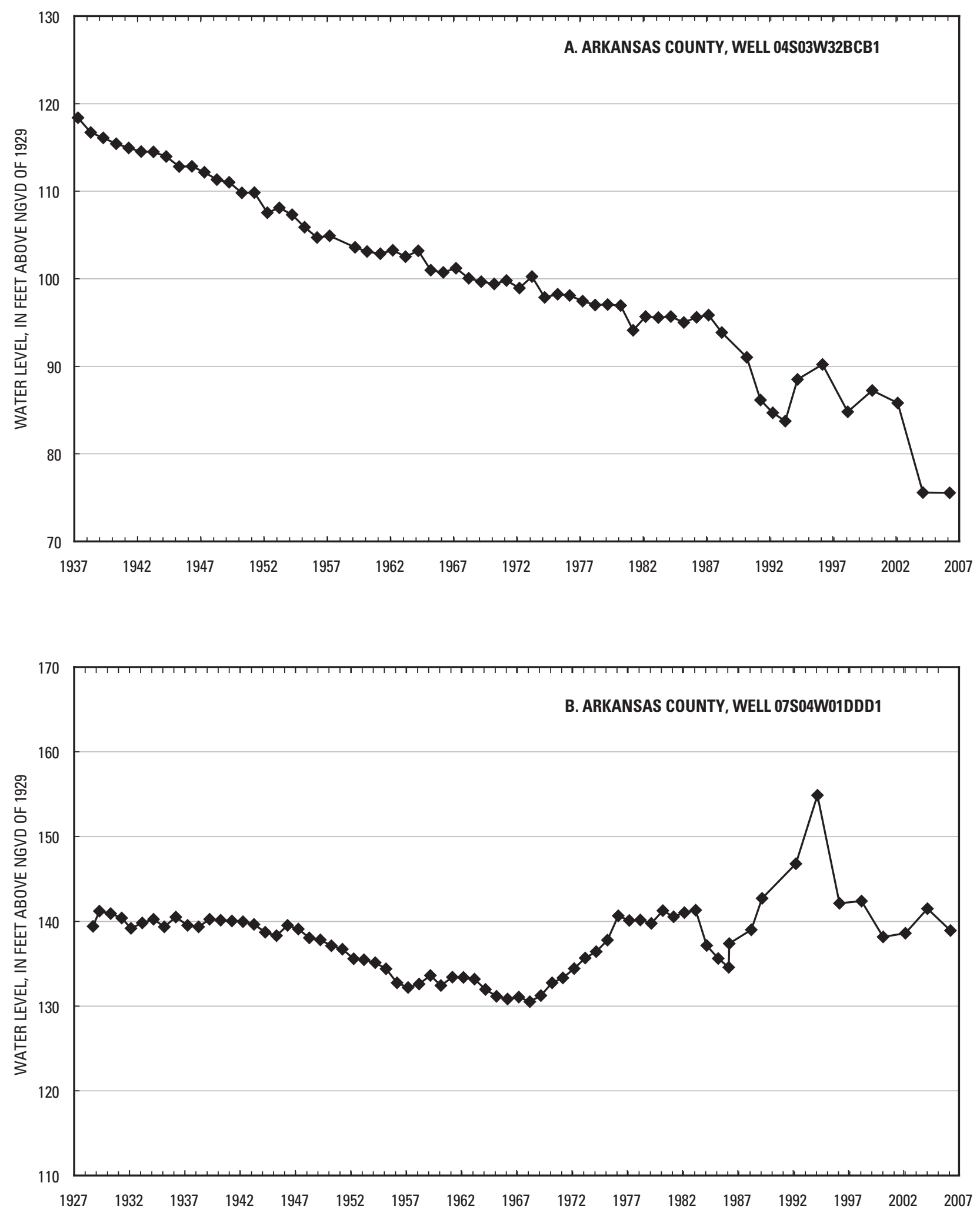

Figure 4. Water-level hydrographs (A to CC) for selected wells in the Mississippi River Valley alluvial aquifer. 

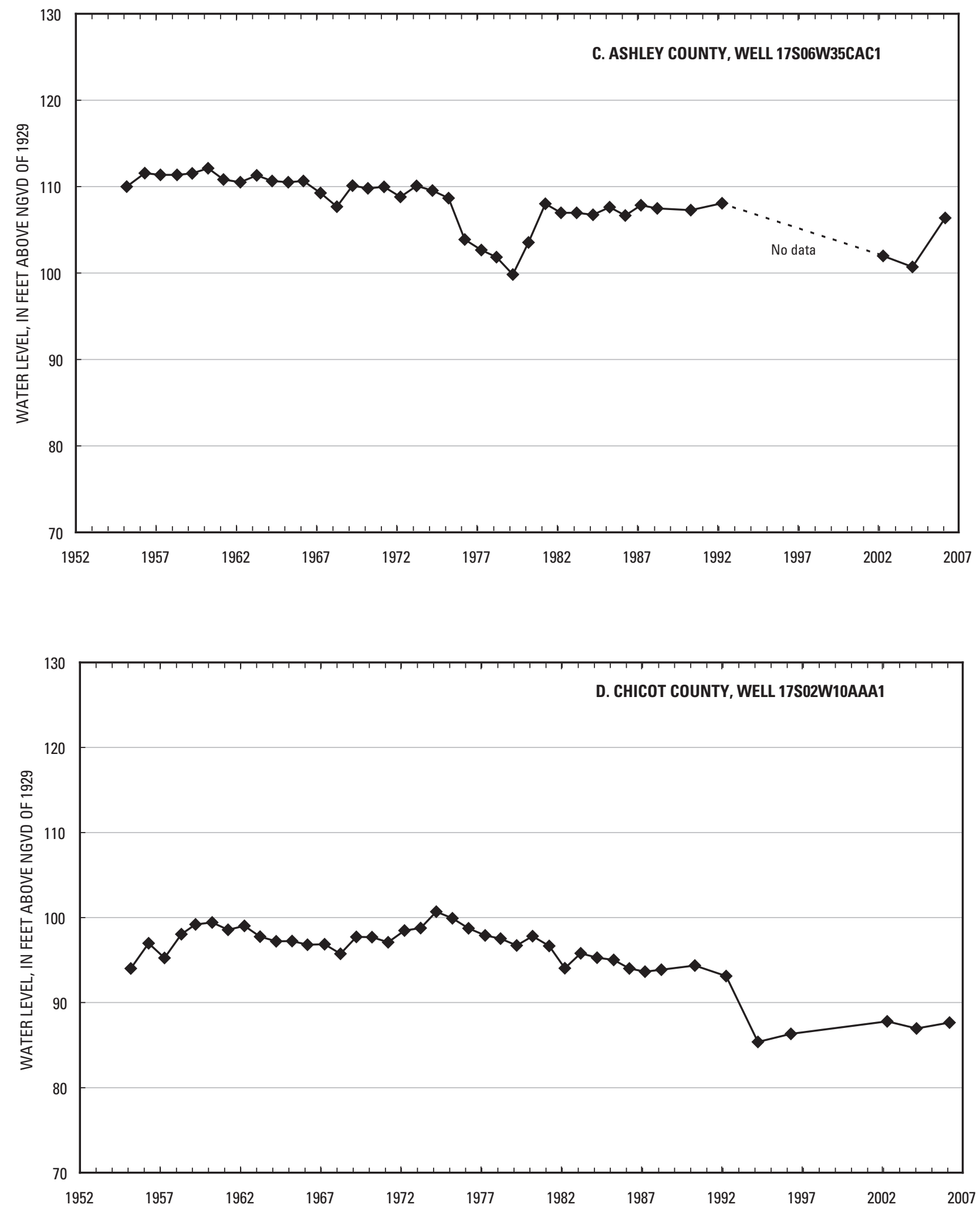

Figure 4. Water-level hydrographs (A to CC) for selected wells in the Mississippi River Valley alluvial aquifer.—Continued 

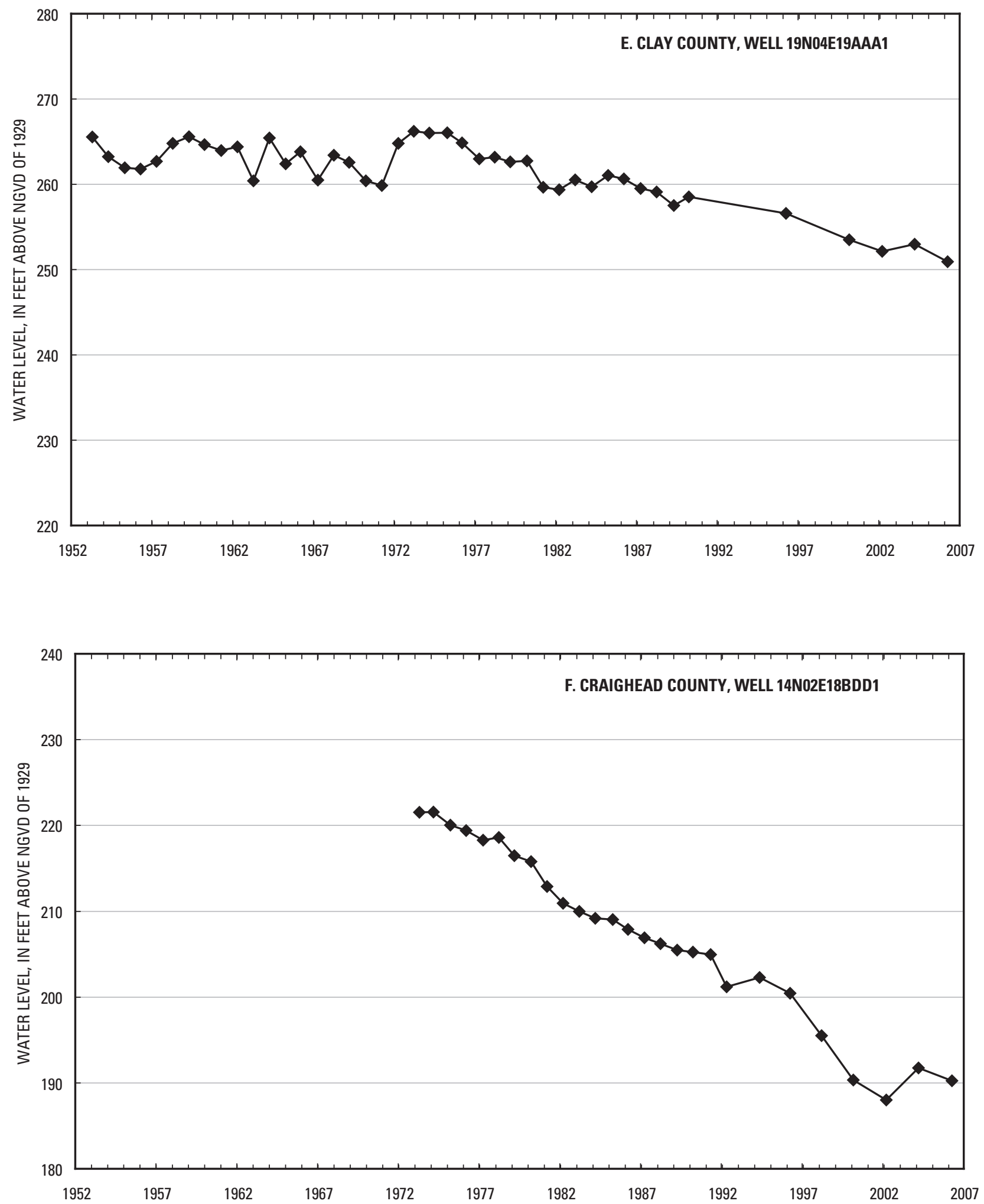

Figure 4. Water-level hydrographs (A to CC) for selected wells in the Mississippi River Valley alluvial aquifer-Continued 

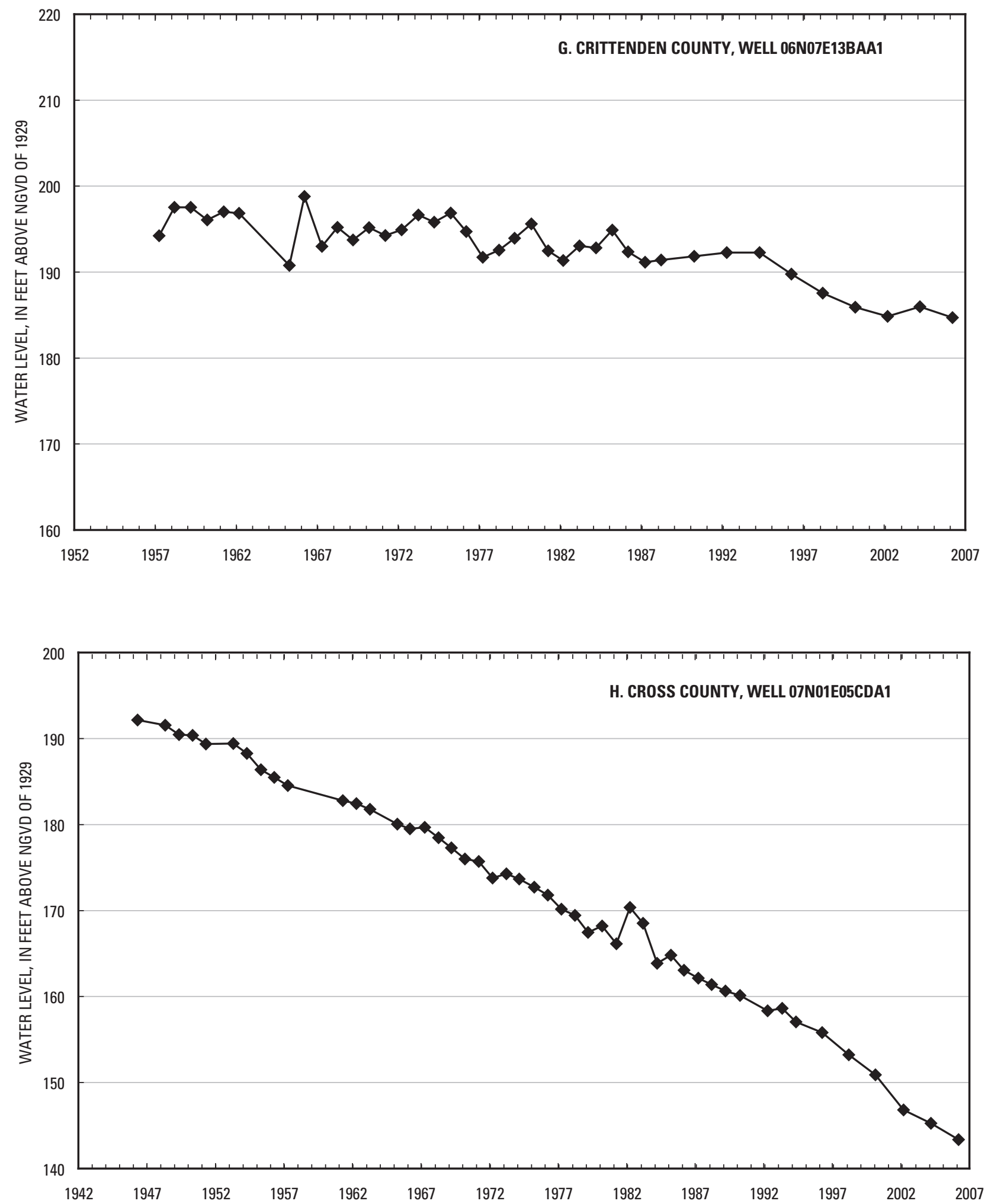

Figure 4. Water-level hydrographs (A to CC) for selected wells in the Mississippi River Valley alluvial aquifer-_Continued 

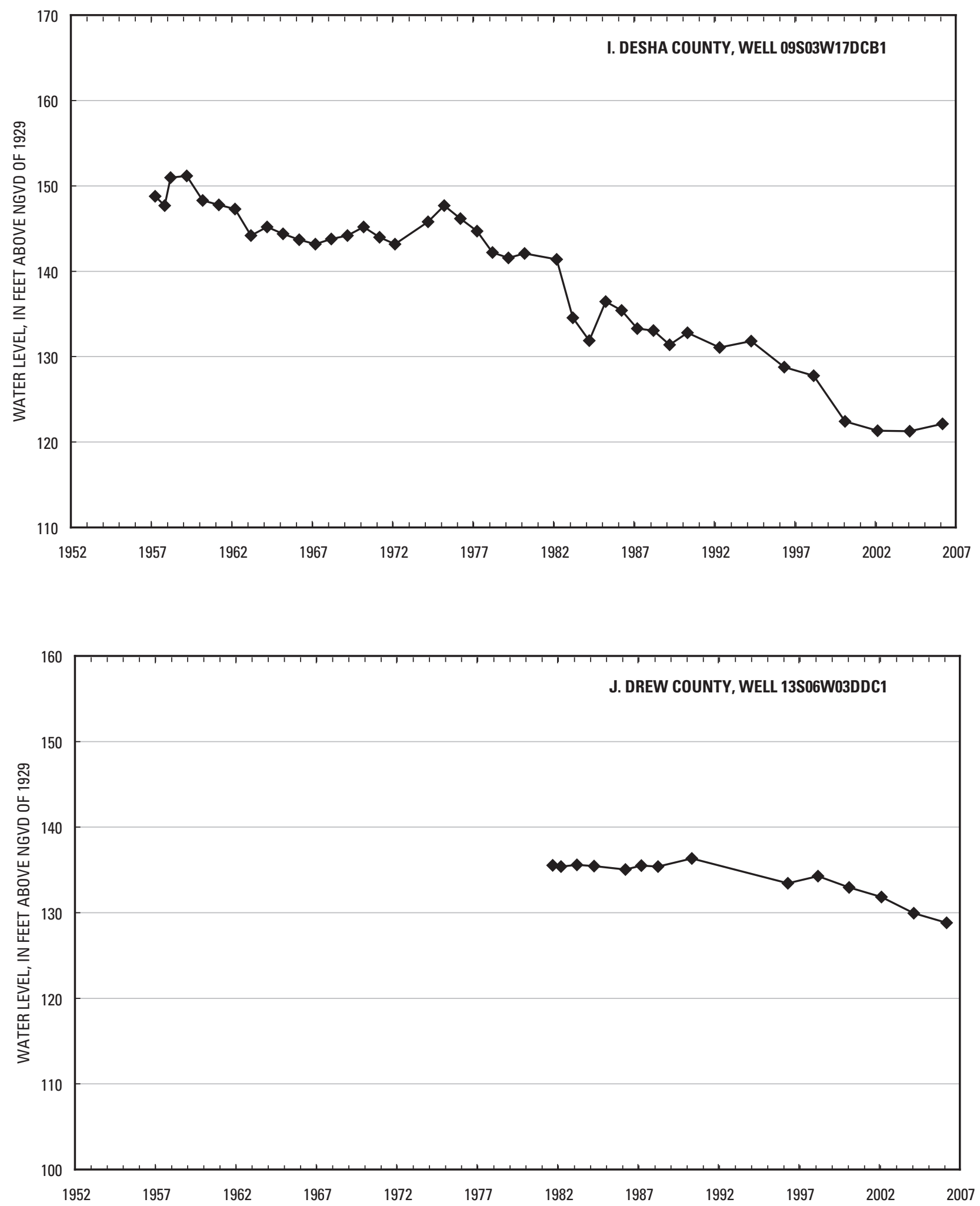

Figure 4. Water-level hydrographs (A to CC) for selected wells in the Mississippi River Valley alluvial aquifer-Continued 

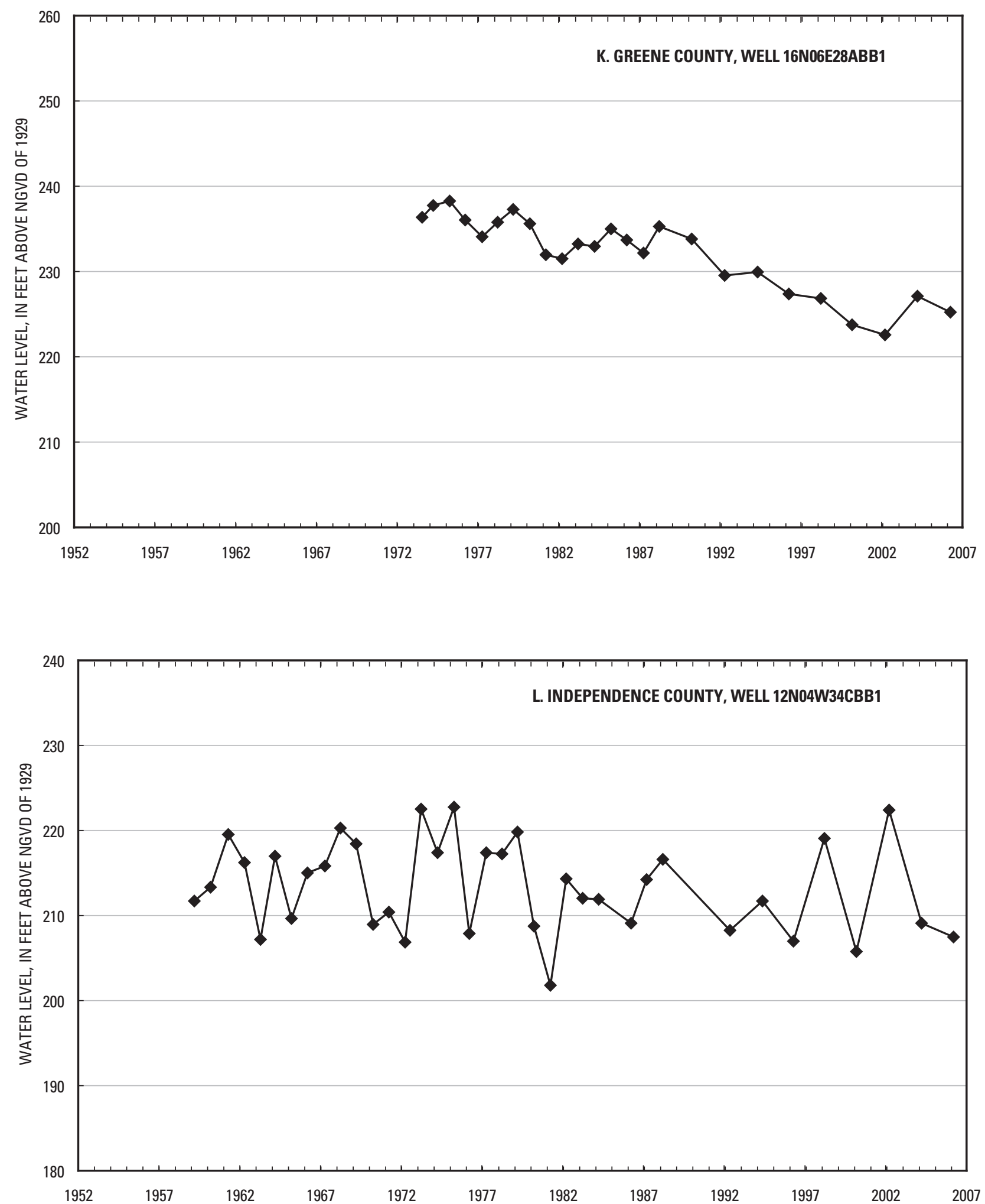

Figure 4. Water-level hydrographs (A to CC) for selected wells in the Mississippi River Valley alluvial aquifer.—Continued 

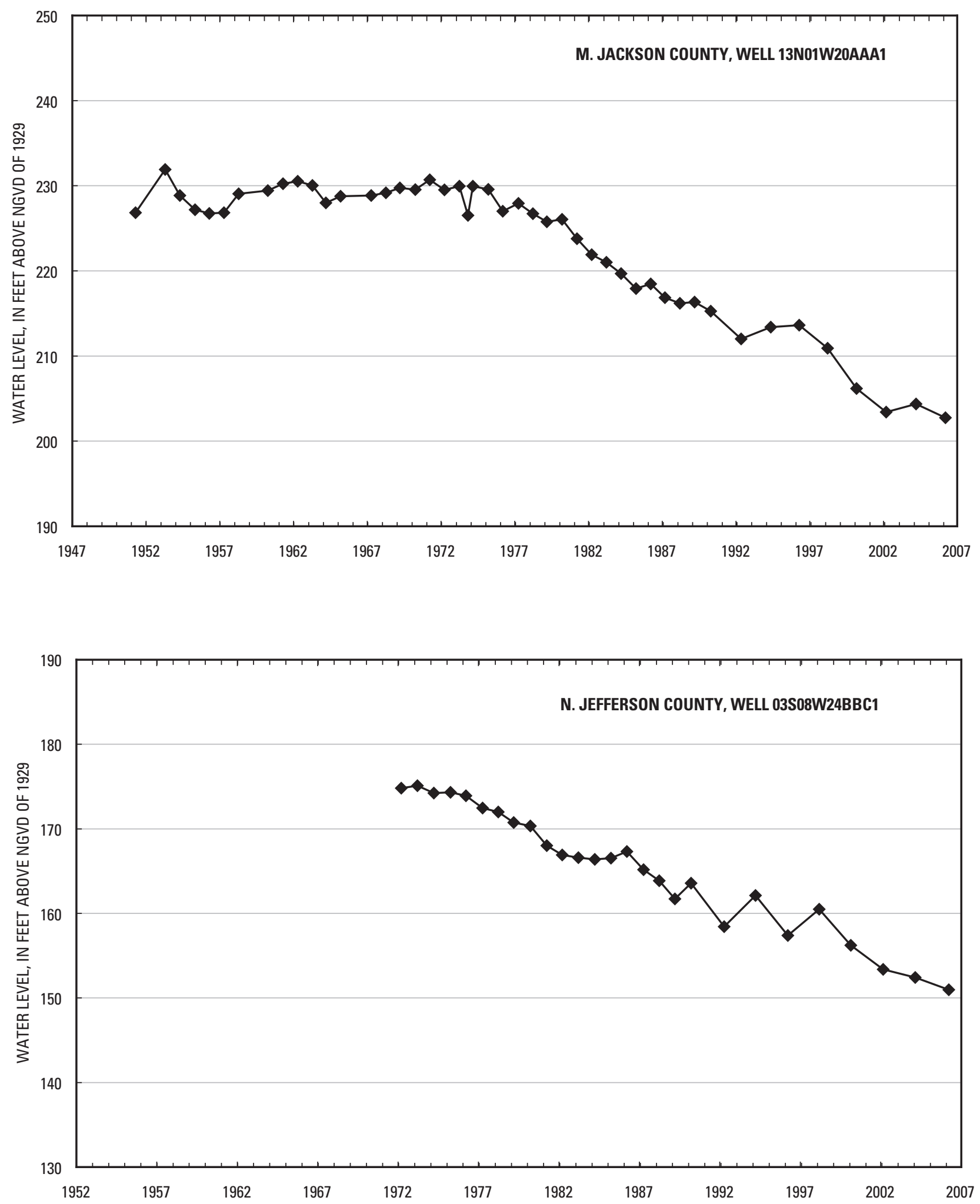

Figure 4. Water-level hydrographs (A to CC) for selected wells in the Mississippi River Valley alluvial aquifer-Continued 

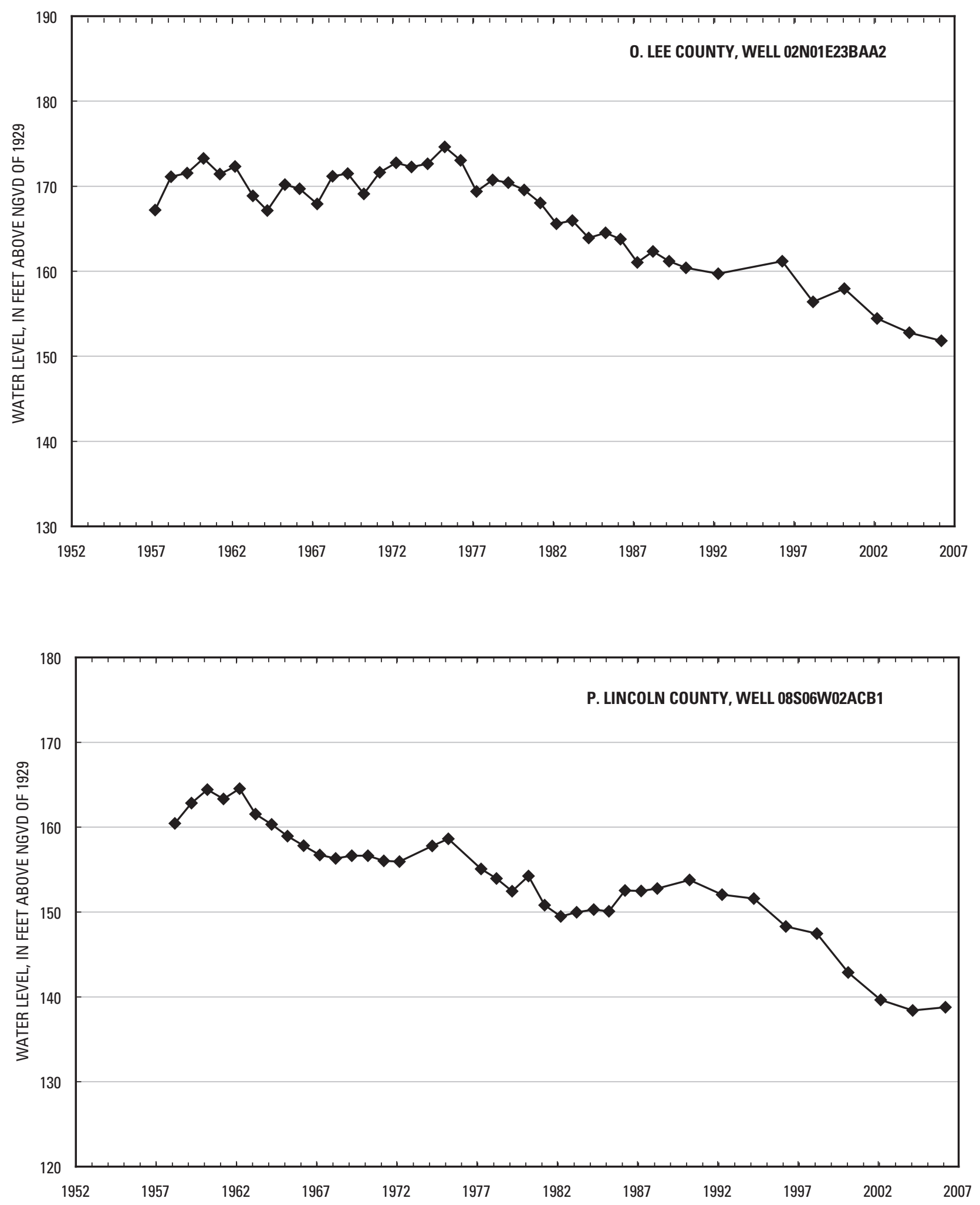

Figure 4. Water-level hydrographs (A to CC) for selected wells in the Mississippi River Valley alluvial aquifer-C Continued 

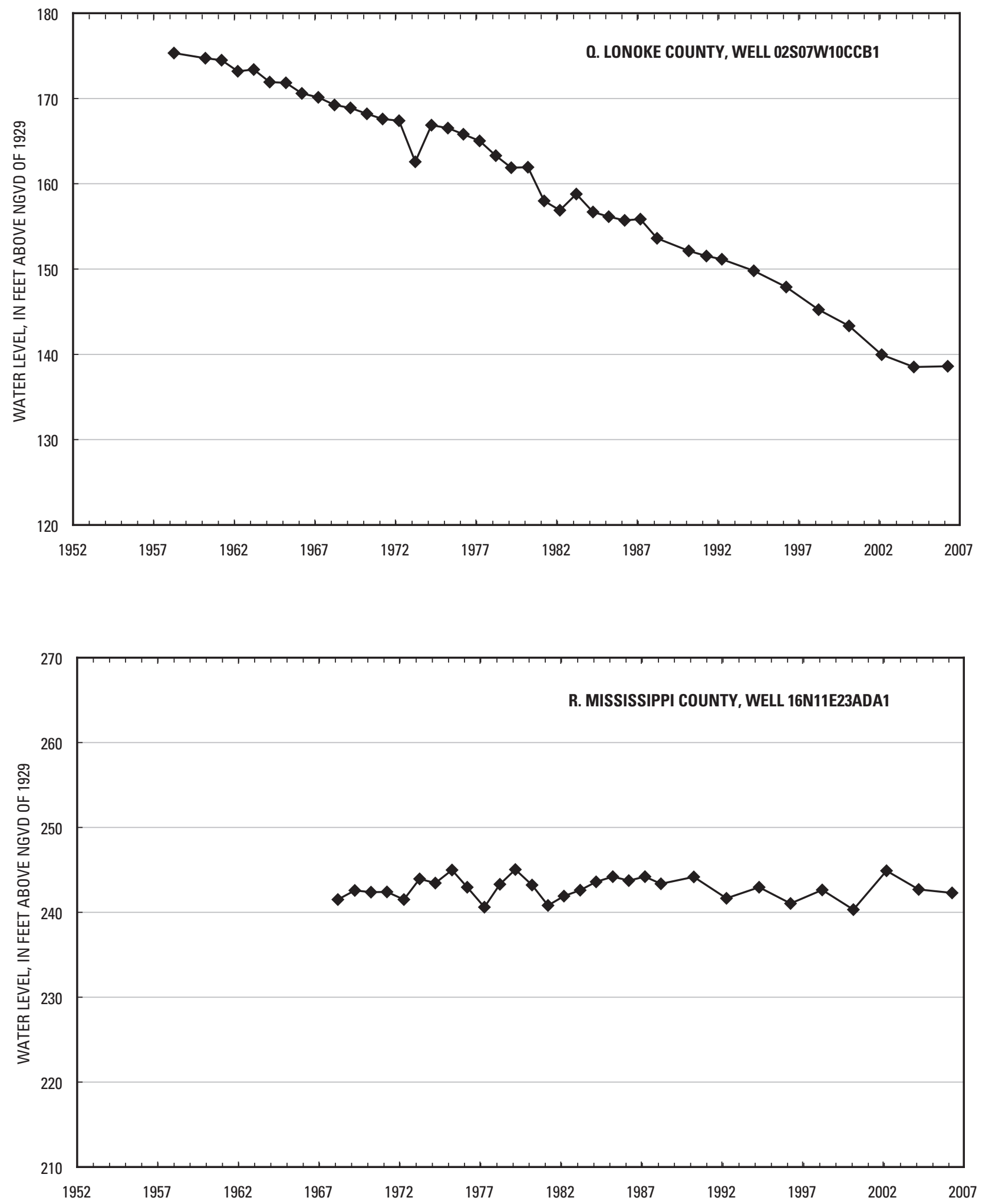

Figure 4. Water-level hydrographs (A to CC) for selected wells in the Mississippi River Valley alluvial aquifer-Continued 

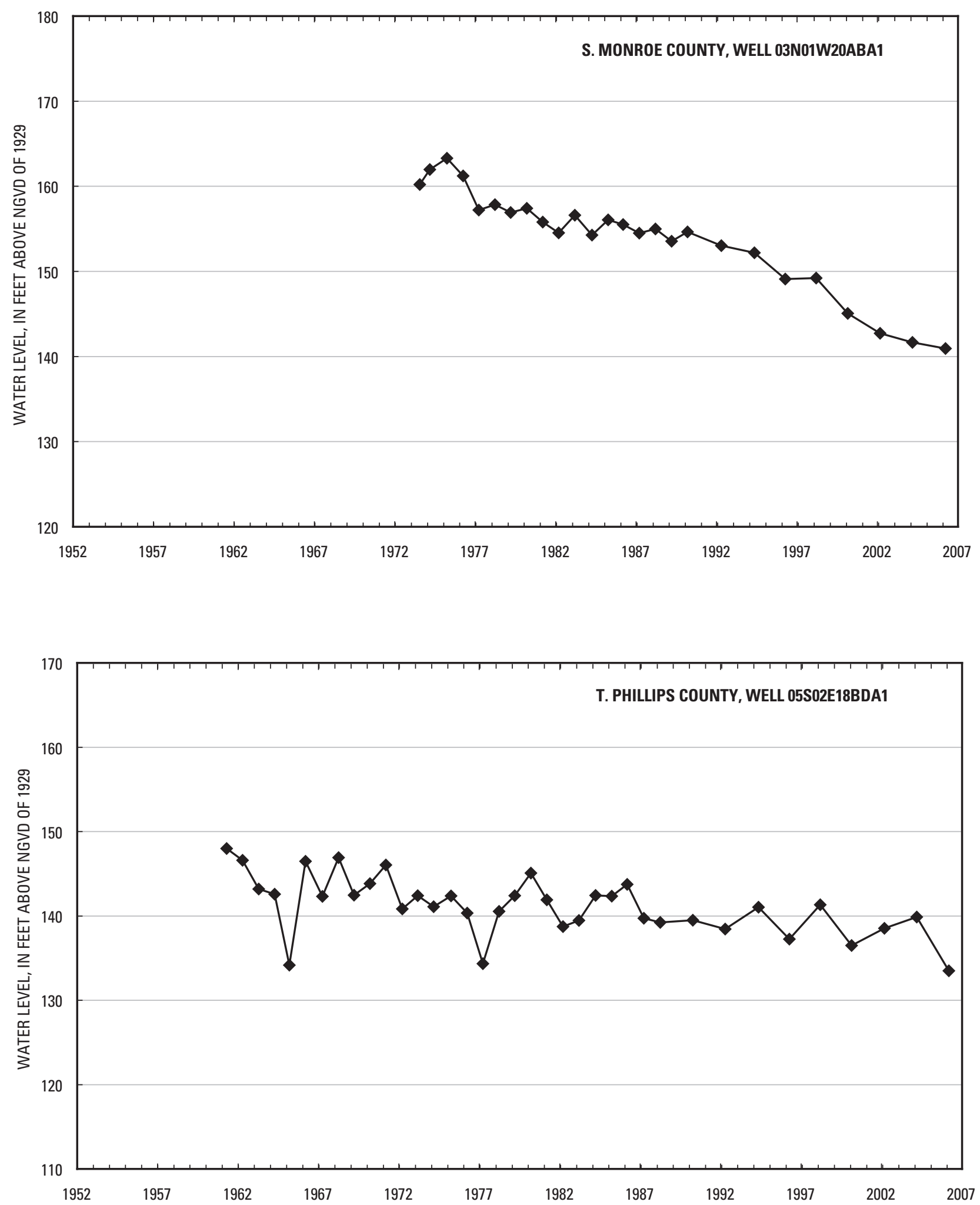

Figure 4. Water-level hydrographs (A to CC) for selected wells in the Mississippi River Valley alluvial aquifer.—Continued 

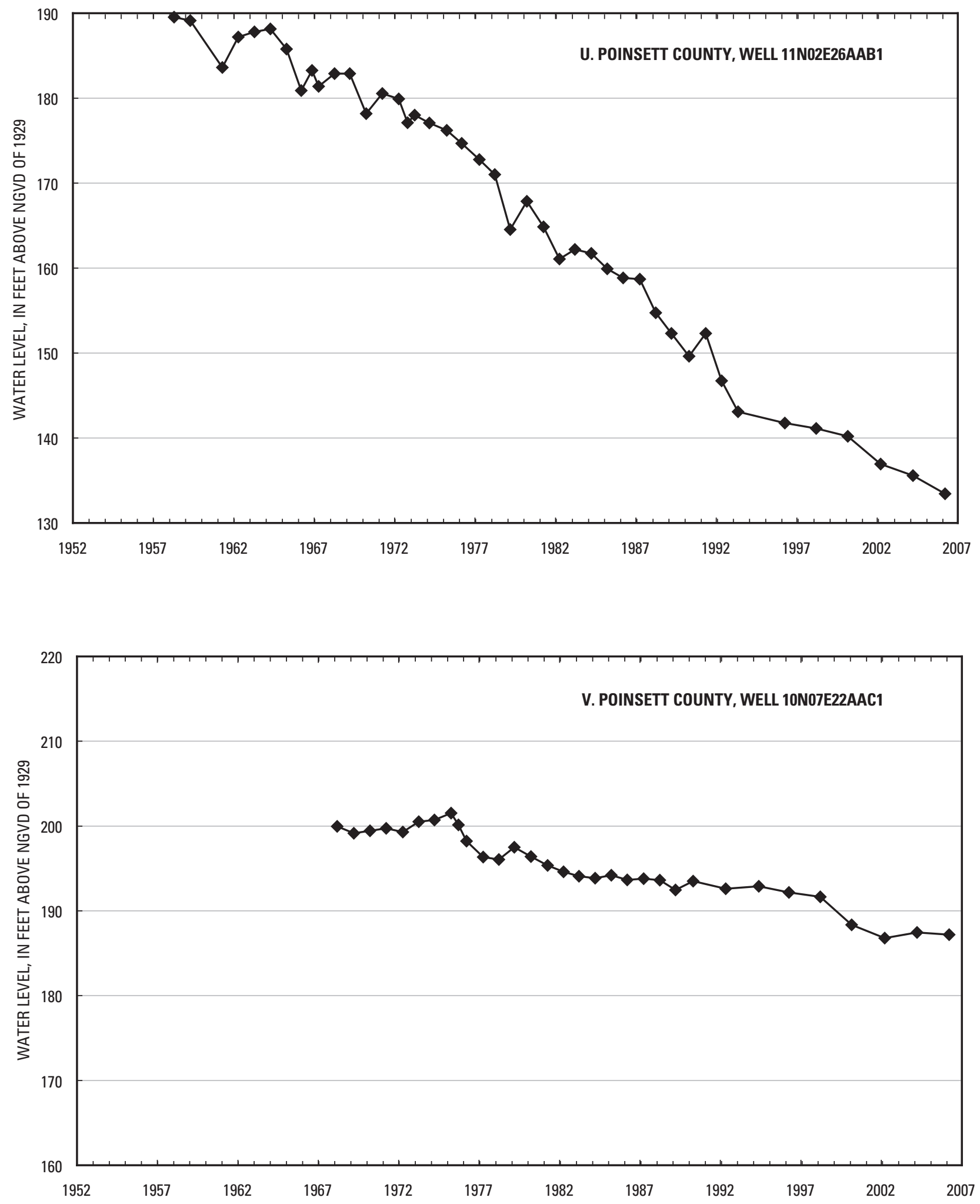

Figure 4. Water-level hydrographs (A to CC) for selected wells in the Mississippi River Valley alluvial aquifer-—Continued 



Figure 4. Water-level hydrographs (A to CC) for selected wells in the Mississippi River Valley alluvial aquifer.—Continued 

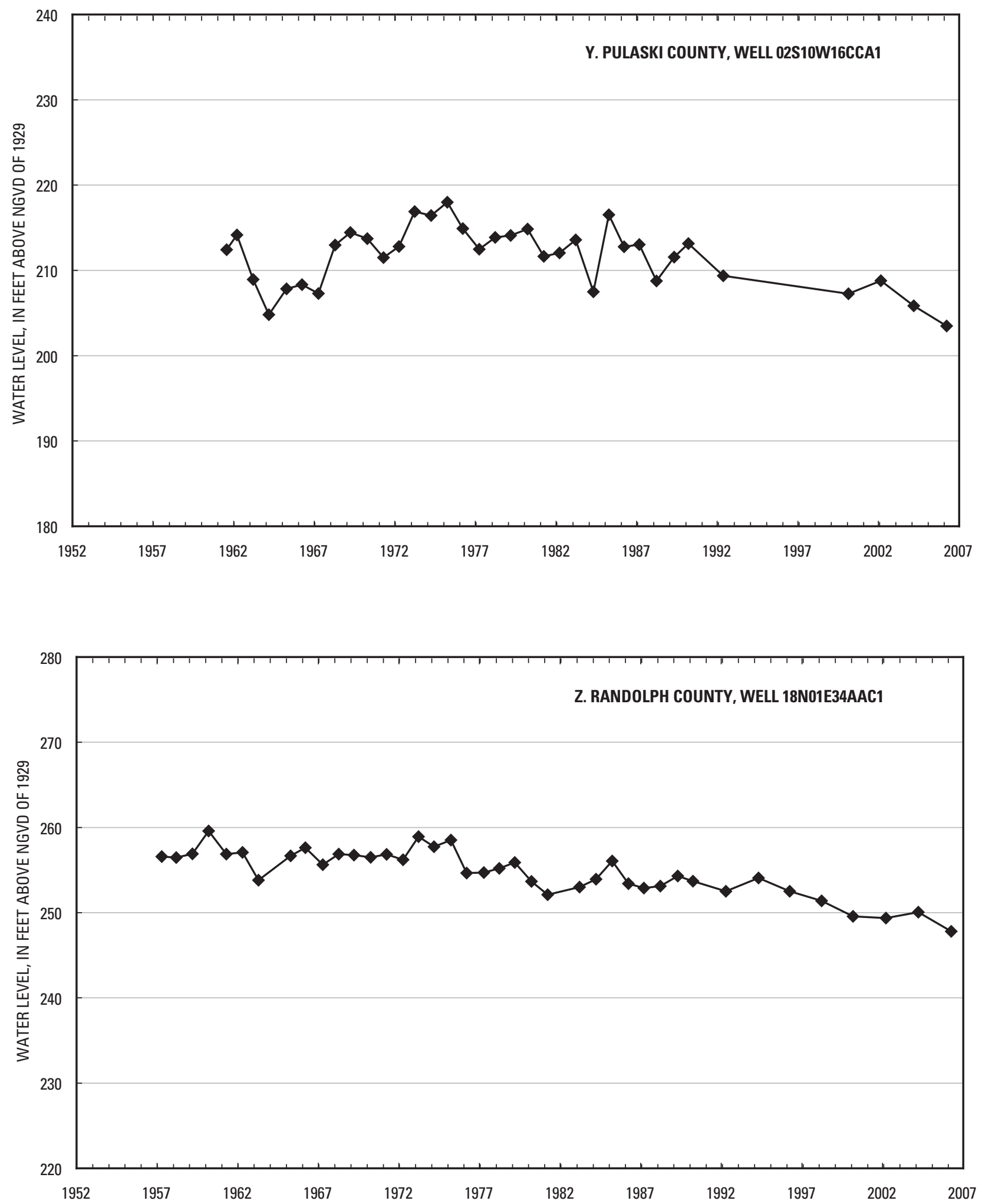

Figure 4. Water-level hydrographs (A to CC) for selected wells in the Mississippi River Valley alluvial aquifer-Continued 

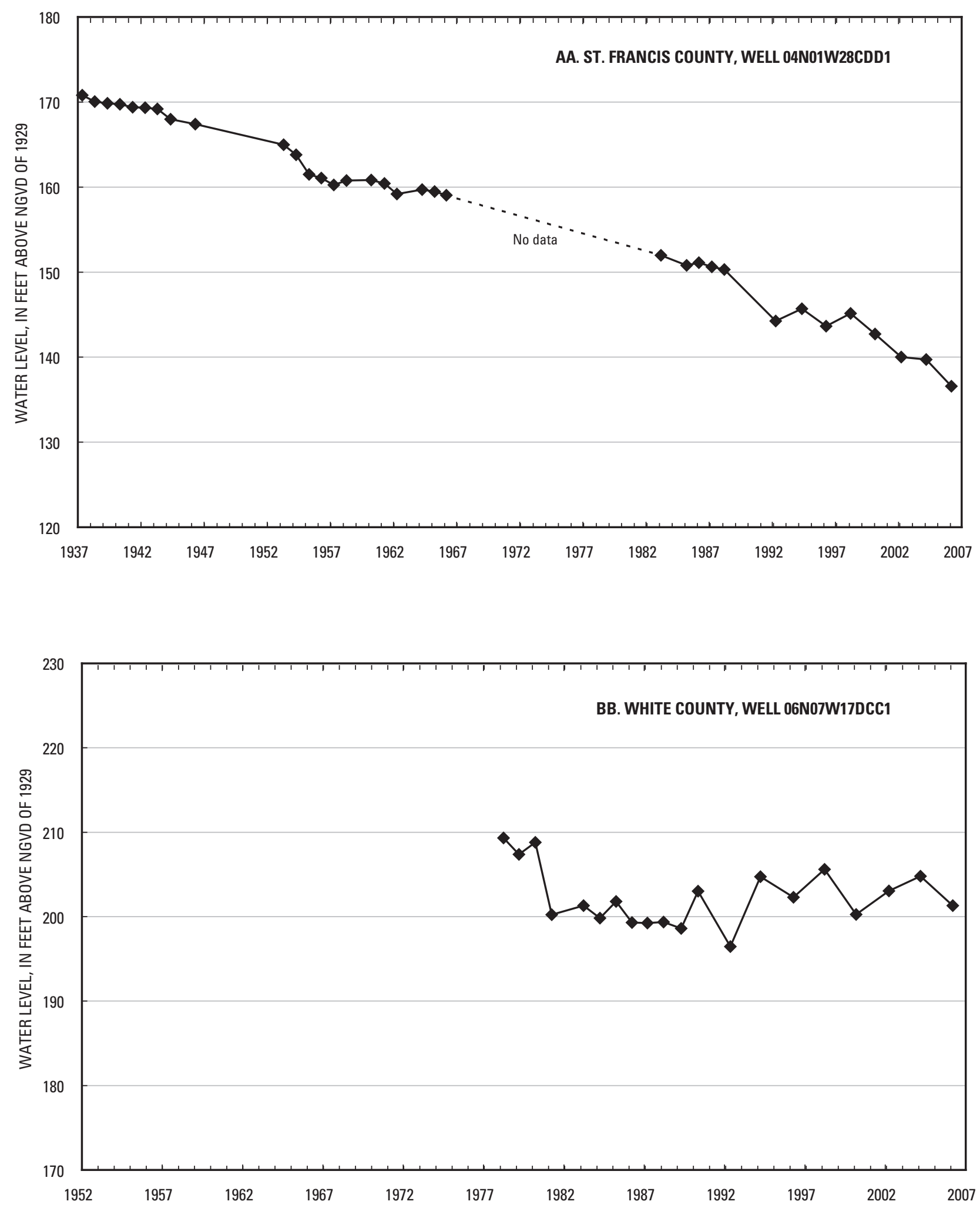

Figure 4. Water-level hydrographs (A to CC) for selected wells in the Mississippi River Valley alluvial aquifer.—Continued 


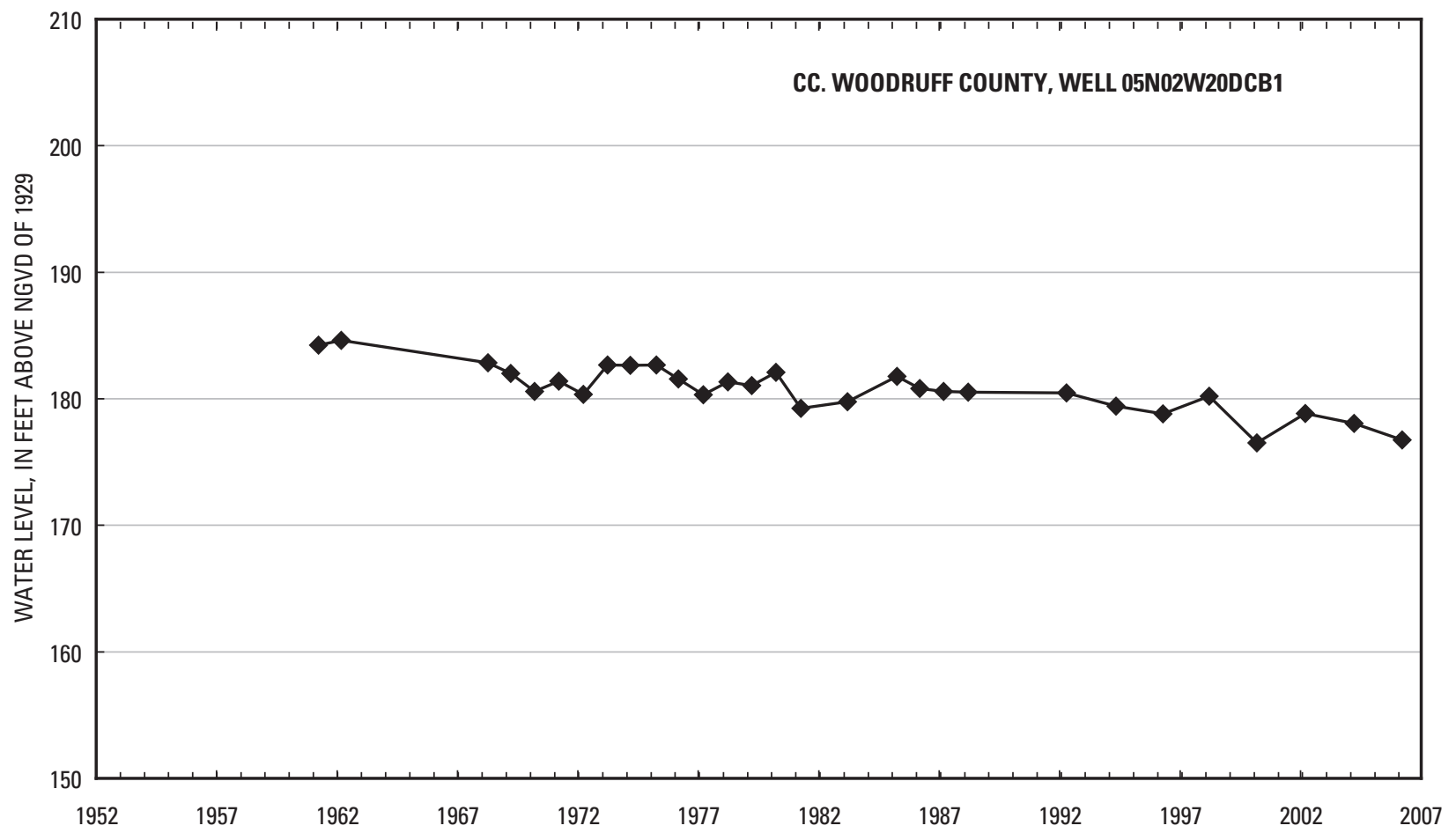

Figure 4. Water-level hydrographs (A to CC) for selected wells in the Mississippi River Valley alluvial aquifer-CContinued 


\section{Water-Quality Conditions}

Water samples were collected from 65 wells completed in the alluvial aquifer and measured onsite for specific conductance and temperature (appendix 3). Specific conductance ranged from 267 microsiemens per centimeter at 25 degrees Celsius $(\mu \mathrm{S} / \mathrm{cm})$ at a well in Clay County to $2,960 \mu \mathrm{S} / \mathrm{cm}$ at a well in Chicot County (appendix 3). The majority of the values are in the 401-600 and 601-800 $\mu \mathrm{S} / \mathrm{cm}$ ranges (fig. 5). Four areas of relatively high specific conductance (greater than or equal to $1,000 \mu \mathrm{S} / \mathrm{cm}$ ) occur in Arkansas, Chicot, Craighead, and Prairie Counties. Other values in Chicot County are as low as $376 \mu \mathrm{S} / \mathrm{cm}$.

Generally, the occurrences of higher specific conductance in the alluvial aquifer probably are caused by movement of water containing elevated concentrations of dissolved solids from sources at depth (Bryant and others, 1985). Water with higher concentrations of dissolved solids may have moved upward where the confining units are thin or absent, along faults, or through unplugged or deteriorated casings of abandoned oil and gas test wells (Fitzpatrick, 1985). Morris and Bush (1986) cite two possible sources of high dissolved-solids concentrations - a zone of ground-water stagnation present in the alluvial aquifer caused by localized restricted horizontal or vertical flow, and upward movement of water with higher dissolved-solids concentration from deeper formations in response to pumping.

\section{Summary}

The Mississippi River Valley alluvial aquifer is increasingly relied upon for agriculture and aquaculture in eastern Arkansas. Since 1965, withdrawals from the alluvial aquifer have increased from about 1,063 Mgal/d to about 7,252 $\mathrm{Mgal} / \mathrm{d}$ in 2005, an increase of about 582 percent. Withdrawals have more than doubled in the last 20 years, about a 105 percent increase since 1985.

Ground-water levels are affected by ground-water withdrawals within the study area, resulting in depressions. In 2006, the lowest water-level altitude was $76 \mathrm{ft}$ in the center of Arkansas County. The highest water-level altitude was 289 ft in northeastern Clay County on the west side of Crowleys Ridge. The elongated depression in Arkansas, Lonoke, and Prairie Counties has two areas that have changed in horizontal extent or depth when compared to previous conditions of the

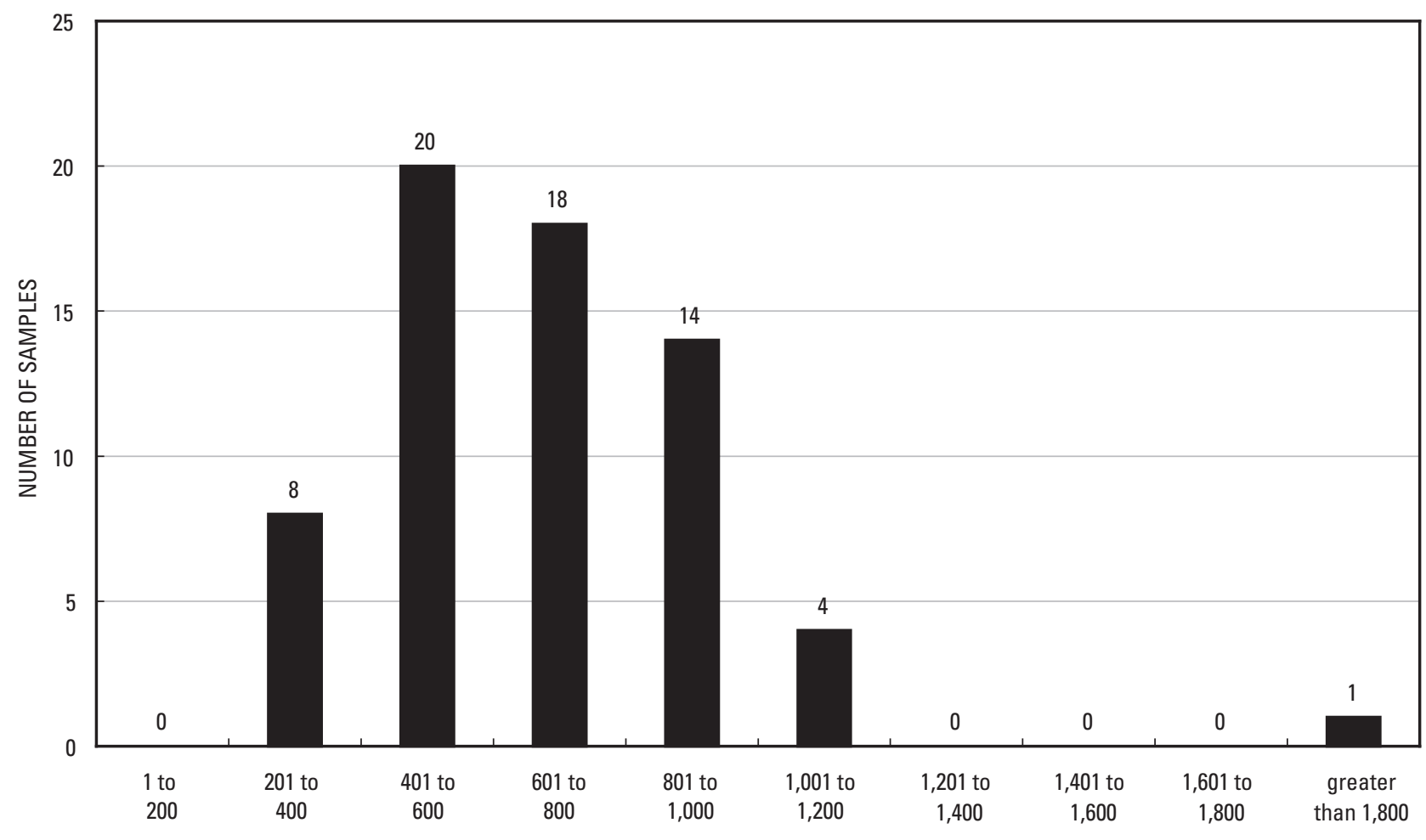

SPECIFIC CONDUCTANCE, IN MICROSIEMENS PER CENTIMETER

Figure 5. Distribution of specific conductance in samples from wells completed in the Mississippi River Valley alluvial aquifer, 2006. 
alluvial aquifer. The area in Arkansas County at the southeastern half of the depression has not expanded horizontally from recent years, although the center of the depression has deepened. The Arkansas and White Rivers, which bound Arkansas County on the southwestern and eastern county lines, are hydrologically connected and provide recharge to the alluvial aquifer. The area in Lonoke and Prairie Counties in the northwestern half of the depression has expanded horizontally in the deeper part of the depression. The 90-foot contour has expanded north and east in Lonoke County when compared with the 2004 potentiometric surface.

Along the west side of Crowleys Ridge, the two previously documented areas of depression expanded and coalesced into a single depression by 2002 . The 2006 potentiometricsurface map shows little change in the area of this depression, although the deeper areas within the depression have expanded. The area in Lee, Monroe, St. Francis, and Woodruff Counties was similar in area to the 2004 potentiometric-surface map. The area in Cross and Poinsett Counties in 2004 has expanded north into Craighead County and east to intersect with Crowleys Ridge in the 2006 potentiometric-surface map.

Three areas of reduced water level were identified in previous work in southeastern Arkansas-one in eastern Lincoln County, a second that extends from southern Desha County into northern Chicot County, and a third that extends from western Chicot County into eastern Ashley County. The area of reduced water level in southern Desha and northern Chicot Counties has expanded further north into Desha County and south into Chicot County in 2006. The area in eastern Lincoln County has expanded into northwestern Desha County and westward in Lincoln County in 2006, with a measured altitude of $119 \mathrm{ft}$ as in 2004. In 2006, the depth of the area in western Chicot and eastern Ashley Counties has not increased and is approximately the same as in the 2004 potentiometric surface. An area of reduced water level in Greene County has expanded and deepened.

Six depressions are shown in the 2006 potentiometricsurface map that are not shown in previous alluvial aquifer potentiometric surfaces. A depression at the Prairie and White County line, a second depression at the Craighead and Mississippi County line, and four small depressions are located in northern Desha, southeastern Jefferson, northern Lee, and northeastern St. Francis Counties. Continued monitoring of the potentiometric surface will determine if these depressions are the result of short-term variations or long-term changes in the hydrologic conditions in the alluvial aquifer.

The regional direction of ground-water flow is generally to the south and east except where flow is affected by groundwater withdrawals; however, the flow direction is affected over substantial areas by depressions. West of Crowleys Ridge, depressions in Arkansas, Lonoke, and Prairie Counties capture ground-water flow from all direction. The flow along large sections of the Arkansas, Mississippi, and White Rivers is away from the rivers.

A map showing the difference in water level was constructed using 645 differences in water levels measured in
633 wells during 2006 and 2002. The difference in measured water levels from 2002 to 2006 ranged from $-24.0 \mathrm{ft}$ to $25.0 \mathrm{ft}$, with a mean of $-2.0 \mathrm{ft}$. The largest decline of $-24.0 \mathrm{ft}$ occurred in Poinsett County and the largest rise of $25.0 \mathrm{ft}$ occurred in Randolph County. Out of the 645 differences, 481 were declines (74.6 percent), 12 were no difference (values of 0.0 ft) (1.8 percent), and 152 were rises ( 23.6 percent). The three areas that have the most declines are west of Crowleys Ridge, eastern Craighead and Mississippi Counties, and in eastern Lonoke and Prairie Counties. Five areas are dominated by rises in measured water levels. The largest area is in western and southern Arkansas, southeastern Jefferson, and northern Desha Counties adjacent to the Arkansas River. Other areas are in eastern Cross and northern Crittenden Counties, western Lonoke County, southeastern White County, and adjacent to the eastern boundary of Crowleys Ridge in Craighead and Greene Counties.

Long-term water-level trends were evaluated using hydrographs from 152 wells in the alluvial aquifer for the period 1982 to 2006 . The mean annual rise or decline in water level for the entire study area was $-0.32 \mathrm{ft} / \mathrm{yr}$ with a range of -1.28 to $0.77 \mathrm{ft} / \mathrm{yr}$. Independence and White Counties are the only counties with a mean annual rise from 1982 to 2006. Mean annual declines between $-0.50 \mathrm{ft} / \mathrm{yr}$ and $0.00 \mathrm{ft} / \mathrm{yr}$ occurred in Arkansas, Ashley, Chicot, Clay, Craighead, Crittenden, Drew, Jefferson, Lee, Mississippi, Monroe, Phillips, Poinsett, Prairie, Pulaski, Randolph, and Woodruff Counties. Mean annual declines between $-1.00 \mathrm{ft} / \mathrm{yr}$ and $-0.50 \mathrm{ft} / \mathrm{yr}$ occurred in Cross, Desha, Greene, Jackson, Lincoln, Lonoke, and St. Francis Counties.

The analysis of long-term water-level changes (1982 to 2006)in Arkansas, Lonoke, and Prairie Counties shows the elongation of the depression in these three counties. Arkansas and Prairie Counties have two different rates of annual decline for the two hydrographs shown for each county. Water levels in the two wells near the Arkansas and White Rivers have risen or declined at a slower rate than in the three wells in the center, northern, and western part of the depression. These rates of water-level change indicate that this depression is expanding in an elongated direction north and west into Lonoke and Prairie Counties. The depression west of Crowleys Ridge has five wells with hydrographs in or near the depression that can be used to characterize the rates of waterlevel change within the depression.

Water samples were collected from 65 wells completed in the alluvial aquifer and measured onsite for specific conductance and temperature. Specific conductance ranged from 267 $\mu \mathrm{S} / \mathrm{cm}$ at a well in Clay County to $2,960 \mu \mathrm{S} / \mathrm{cm}$ at a well in Chicot County. Four areas of relatively high specific conductance (greater than or equal to $1,000 \mu \mathrm{S} / \mathrm{cm}$ ) occur in Arkansas, Chicot, Craighead, and Prairie Counties. 


\section{Selected References}

Ackerman, D.J., 1996, Hydrology of the Mississippi River Valley alluvial aquifer, south-central United States-A preliminary assessment of the regional flow system: U.S. Geological Survey Professional Paper 1416-D, 56 p.

Boswell, E.H., Cushing, E.M., and Hosman, R.L., 1968, Quaternary aquifers in the Mississippi Embayment with a discussion of Quality of the water by H.G. Jeffery: U.S. Geological Survey Open Professional Paper 448-E, 15 p.

Broom, M.E. and Reed, J.E., 1973, Hydrology of the Bayou Bartholomew alluvial aquifer-stream system, Arkansas: U.S. Geological Survey Open-File Report 73-34, 91 p.

Bryant, C.T., Ludwig, A.H., and Morris, E.E., 1985, Ground water problems in Arkansas: U.S. Geological Survey WaterResources Investigations Report 85-4010, 24 p.

Fenneman, N.M., 1938, Physiography of eastern United States; New York, McGraw-Hill Book Co. Inc., 689 p.

Freiwald, D.A., 1984, Average annual precipitation and runoff for Arkansas, 1951-80: U.S. Geological Survey Water-Resources Investigations Report 84-4363, 1 sheet.

Fitzpatrick, D.J., 1985, Occurrence of saltwater in the alluvial aquifer in the Boeuf-Tensas Basin, Arkansas: U.S. Geological Survey Water-Resources Investigation Report 85-4029, 1 sheet.

Gonthier, G.J., and Mahon, G.L., 1993, Thickness of the Mississippi River Valley confining unit, eastern Arkansas: U.S. Geological Survey Water-Resources Investigation Report 92-4121, 4 sheets.

Halberg, H.N., 1972, Use of water in Arkansas, 1970: Arkansas Geological Commission Water Resources Summary Number 7, 17 p.

Halberg, H.N., 1977, Use of water in Arkansas, 1975: Arkansas Geological Commission Water Resources Summary Number 9, 28 p.

Halberg, H.N., and Stephens, J.W., 1966, Use of water in Arkansas, 1965: Arkansas Geological Commission Water Resources Summary Number 5, 12 p.

Helsel, D.R., and Hirsch, R.M., 1992, Statistical methods in water resources: New York, NY, Elsevier Science Publishing Co., 522 p.

Holland, T.W., 1987, Use of water in Arkansas, 1985: Arkansas Geological Commission Water Resources Summary Number 14, $30 \mathrm{p}$.

Holland, T.W., 1993, Use of water in Arkansas, 1990: U.S. Geological Survey Open-File Report 93-48, pamphlet.
Holland, T.W., 1999, Water use in Arkansas, 1995: U.S. Geological Survey Open-File Report 99-188, 1 sheet.

Holland, T.W., 2004, Estimated water use in Arkansas, 2000: U.S. Geological Survey Scientific Investigations Report 2004-5230, $31 \mathrm{p}$.

Holland, T.W., and Ludwig, A.H., 1981, Use of water in Arkansas, 1980: Arkansas Geological Commission Water Resources Summary Number 14, 30 p.

Joseph, R.L., 1999, Status of water levels and selected waterquality conditions in the Mississippi River Valley alluvial aquifer in eastern Arkansas, 1998: U.S. Geological Survey Water-Resources Investigations Report 99-4035, 54 p.

Mahon, G.L., and Poynter, D.T., 1993, Development, calibration, and testing of ground-water flow models for the Mississippi River Valley alluvial aquifer in eastern Arkansas using one-square mile cells: U.S. Geological Survey WaterResources Investigations Report 92-4106, 33 p.

Morris, E.E., and Bush, W.V., 1986, Extent and source of saltwater intrusion into the alluvial aquifer near Brinkley, Arkansas, 1984: U.S. Geological Survey Water-Resources Investigations Report 85-4322, 123 p.

Pugh, A.L., Westerfield, P.W., and Poynter, D.T., 1997, Thickness of the Mississippi River Valley alluvial aquifer in eastern Arkansas: U.S. Geological Survey Water-Resources Investigations Report 97-4049, 1 sheet.

Reed, T.B., 2004, Status of water levels and selected waterquality conditions in the Mississippi River Valley alluvial aquifer in eastern Arkansas, 2002: U.S. Geological Survey Scientific Investigations Report 2004-5129, 53 p.

Schrader, T.P., 2001, Status of water levels and selected waterquality conditions in the Mississippi River Valley alluvial aquifer in eastern Arkansas, 2000: U.S. Geological Survey Water-Resources Investigations Report 01-4124, 52 p.

Schrader, T.P., 2006, Status of water levels and selected waterquality conditions in the Mississippi River Valley alluvial aquifer in eastern Arkansas, 2004: U.S. Geological Survey Scientific Investigations Report 2006-5128, 82 p.

Stanton, G.P., Joseph, R.L., and Pugh, A.L., 1998, Status of water levels and selected water-quality conditions in the Mississippi River Valley alluvial aquifer in eastern Arkansas, 1994-1996: U.S. Geological Survey Water-Resources Investigations Report 98-4131, 72 p. 


\section{Appendixes 1-3}


Appendix 1. Information pertaining to water levels measured in wells completed in the Mississippi River Valley alluvial aquifer in eastern Arkansas, spring 2006.

[USGS, U.S. Geological Survey; NRCS, Natural Resources Conservation Service; --, no data; NGVD of 1929, National Geodetic Vertical Datum of 1929; Horizontal coordinate information is referenced to the North American Datum of 1983 (NAD 83); *, control wells for duplicate measurements by USGS and NRCS]

\begin{tabular}{|c|c|c|c|c|c|c|c|c|}
\hline Station name & $\begin{array}{c}\text { Latitude } \\
\text { (degrees, } \\
\text { minutes, } \\
\text { seconds) }\end{array}$ & $\begin{array}{c}\text { Longitude } \\
\text { (degrees, } \\
\text { minutes, } \\
\text { seconds) }\end{array}$ & $\begin{array}{c}\text { Source } \\
\text { of } \\
\text { data }\end{array}$ & $\begin{array}{c}\text { Depth } \\
\text { of well } \\
\text { (feet) }\end{array}$ & $\begin{array}{l}\text { Land-surface } \\
\text { datum altitude } \\
\text { (feet above } \\
\text { NGVD of } \\
\text { 1929) }\end{array}$ & $\begin{array}{c}\text { Depth to } \\
\text { water } \\
\text { (feet below } \\
\text { land-surface } \\
\text { datum) }\end{array}$ & $\begin{array}{l}\text { Water-level } \\
\text { altitude } \\
\text { (feet above } \\
\text { NGVD of } \\
\text { 1929) }\end{array}$ & $\begin{array}{c}\text { Date of } \\
\text { measure- } \\
\text { ment }\end{array}$ \\
\hline \multicolumn{9}{|c|}{ Arkansas County } \\
\hline 02S04W11DBB1 & 343233 & 912415 & USGS & 152 & 213.04 & 100.70 & 112 & $4 / 10 / 2006$ \\
\hline 02S05W15AAB1 & 343213 & 913127 & USGS & 180 & 213 & 105.10 & 108 & $4 / 10 / 2006$ \\
\hline 03S02W27ABB1 & 342448 & 911251 & USGS & 87 & 197 & 67.53 & 129 & $4 / 06 / 2006$ \\
\hline 03S03W05CCD1 & 342737 & 912132 & USGS & 150 & 201 & 98.18 & 103 & $4 / 10 / 2006$ \\
\hline 03S03W27BBC1 & 342455 & 911944 & USGS & 120 & 195 & 91.65 & 103 & $4 / 10 / 2006$ \\
\hline 03S04W02BBB1 & 342831 & 912454 & USGS & 116 & 197.63 & 92.23 & 105 & $4 / 10 / 2006$ \\
\hline 03S04W03DCA16 & 342753 & 912515 & USGS & 126 & 205 & 100.45 & 105 & $5 / 02 / 2006$ \\
\hline 03S04W03DCA6 & 342753 & 912517 & USGS & 122.3 & 204 & 99.73 & 104 & $4 / 10 / 2006$ \\
\hline 03S04W03DDA1 & 342750 & 912460 & USGS & 127 & 202 & 100.05 & 102 & $4 / 10 / 2006$ \\
\hline 03S05W03CCC1 & 342752 & 913227 & USGS & 110 & 215 & 104.40 & 111 & $4 / 10 / 2006$ \\
\hline 03S06W35ADD1 & 342411 & 913652 & USGS & -- & 190 & 52.89 & 137 & $4 / 07 / 2006$ \\
\hline 04S01W04ACD2 & 342233 & 910733 & USGS & 52.4 & 155 & 5.74 & 149 & $4 / 06 / 2006$ \\
\hline 04S01W31DCB1 & 341753 & 910949 & USGS & 130 & 179 & 52.65 & 126 & $4 / 06 / 2006$ \\
\hline 04S02W11AAA1 & 342209 & 911123 & USGS & -- & 195.08 & 69.10 & 126 & $4 / 06 / 2006$ \\
\hline 04S02W29CCC1 & 341846 & 911539 & USGS & 140 & 191 & 86.52 & 104 & $4 / 06 / 2006$ \\
\hline 04S03W17ADD1 & 342102 & 912058 & USGS & -- & 200 & 107.72 & 92 & $4 / 10 / 2006$ \\
\hline 04S03W32BCB1 & 341820 & 912202 & USGS & -- & 192 & 116.45 & 76 & $4 / 10 / 2006$ \\
\hline 04S04W02ABB1 & 342313 & 912424 & USGS & 155 & 200 & 108.63 & 91 & $4 / 10 / 2006$ \\
\hline 04S04W35ABC1 & 341835 & 912437 & NRCS & -- & 193 & 106 & 87 & $4 / 12 / 2006$ \\
\hline 04S05W16CDC1 & 342045 & 913321 & USGS & 120 & 201 & 69.35 & 132 & 4/07/2006 \\
\hline 04S05W24DAA1 & 342001 & 912930 & USGS & 150 & 198 & 90.01 & 108 & $4 / 10 / 2006$ \\
\hline 04S06W15DBB1 & 342122 & 913827 & USGS & 100 & 190 & 33.06 & 157 & $4 / 07 / 2006$ \\
\hline 05S01W16BAB1 & 341552 & 910729 & USGS & -- & 183 & 51.05 & 132 & 4/06/2006 \\
\hline 05S02W16ABD1 & 341552 & 911358 & USGS & 154 & 190 & 83.30 & 107 & $4 / 06 / 2006$ \\
\hline 05S04W07CCC1 & 341555 & 912932 & USGS & 120 & 194 & 74.27 & 120 & $4 / 06 / 2006$ \\
\hline 05S04W32BBA1 & 341316 & 912822 & USGS & -- & 191 & 57.42 & 134 & $4 / 06 / 2006$ \\
\hline 05S06W02DDD1 & 341724 & 913651 & USGS & 60 & 182.93 & 20.64 & 162 & $4 / 07 / 2006$ \\
\hline 05S06W07DDC1 & 341642 & 914130 & USGS & 32 & 180.48 & 2.39 & 178 & $4 / 07 / 2006$ \\
\hline 06S02W23DCD1 & 340853 & 911206 & USGS & -- & 188 & 69.74 & 118 & 4/06/2006 \\
\hline 06S03W10BBA1 & 341136 & 911954 & USGS & 155 & 184 & 81.98 & 102 & $4 / 06 / 2006$ \\
\hline 06S03W27AAA1 & 340858 & 911913 & USGS & 132 & 183.14 & 66.68 & 116 & $4 / 06 / 2006$ \\
\hline 07S02W04BBB1 & 340707 & 911452 & USGS & -- & 176 & 42.20 & 134 & 4/06/2006 \\
\hline 07S02W17BBA1 & 340530 & 911539 & USGS & 95 & 184 & 54.51 & 129 & $4 / 06 / 2006$ \\
\hline 07S03W18CCD1 & 340435 & 912316 & USGS & -- & 186.18 & 42.41 & 144 & $4 / 06 / 2006$ \\
\hline
\end{tabular}


Appendix 1. Information pertaining to water levels measured in wells completed in the Mississippi River Valley alluvial aquifer in eastern Arkansas, spring 2006.-Continued

[USGS, U.S. Geological Survey; NRCS, Natural Resources Conservation Service; --, no data; NGVD of 1929, National Geodetic Vertical Datum of 1929; Horizontal coordinate information is referenced to the North American Datum of 1983 (NAD 83); *, control wells for duplicate measurements by USGS and NRCS]

\begin{tabular}{|c|c|c|c|c|c|c|c|c|}
\hline Station name & $\begin{array}{c}\text { Latitude } \\
\text { (degrees, } \\
\text { minutes, } \\
\text { seconds) }\end{array}$ & $\begin{array}{c}\text { Longitude } \\
\text { (degrees, } \\
\text { minutes, } \\
\text { seconds) }\end{array}$ & $\begin{array}{c}\text { Source } \\
\text { of } \\
\text { data }\end{array}$ & $\begin{array}{c}\text { Depth } \\
\text { of well } \\
\text { (feet) }\end{array}$ & $\begin{array}{l}\text { Land-surface } \\
\text { datum altitude } \\
\text { (feet above } \\
\text { NGVD of } \\
\text { 1929) }\end{array}$ & $\begin{array}{c}\text { Depth to } \\
\text { water } \\
\text { (feet below } \\
\text { land-surface } \\
\text { datum) }\end{array}$ & $\begin{array}{l}\text { Water-level } \\
\text { altitude } \\
\text { (feet above } \\
\text { NGVD of } \\
\text { 1929) }\end{array}$ & $\begin{array}{c}\text { Date of } \\
\text { measure- } \\
\text { ment }\end{array}$ \\
\hline \multicolumn{9}{|c|}{ Arkansas County-Continued } \\
\hline 07S03W32BBC1 & 340240 & 912216 & USGS & 128 & 176.92 & 24.72 & 152 & $4 / 06 / 2006$ \\
\hline 07S04W01DDD1 & 340625 & 912327 & USGS & 155 & 186 & 47.10 & 139 & $4 / 06 / 2006$ \\
\hline 08S02W08ACA1 & 340041 & 911506 & USGS & -- & 179 & 45.13 & 134 & $4 / 06 / 2006$ \\
\hline 08S03WT2299 & 340147 & 912203 & USGS & 158 & 178 & 21.47 & 157 & $4 / 06 / 2006$ \\
\hline \multicolumn{9}{|c|}{ Ashley County } \\
\hline 15S04W23DBD1 & 332247 & 912852 & USGS & -- & 128 & 32.00 & 96 & $3 / 15 / 2006$ \\
\hline 15S04W26DCC1 & 332232 & 912902 & USGS & 64.1 & 127 & 31.09 & 96 & $3 / 15 / 2006$ \\
\hline 15S07W21CBA1 & 332316 & 915001 & USGS & 27.4 & 210 & 6.60 & 203 & $3 / 15 / 2006$ \\
\hline 16S06W08CAA1 & 331941 & 914438 & USGS & 105 & 185 & 78.28 & 107 & $3 / 15 / 2006$ \\
\hline 16S06W27BAB1 & 331729 & 914240 & USGS & 115 & 182 & 83.84 & 98 & $3 / 15 / 2006$ \\
\hline 17S04W03ABB1 & 331528 & 913010 & USGS & 105 & 124 & 30.22 & 94 & $3 / 15 / 2006$ \\
\hline 17S04W15DDC1 & 331252 & 912954 & USGS & 57 & 116 & 26.50 & 90 & $3 / 15 / 2006$ \\
\hline 17S04W21ABA1 & 331252 & 913108 & USGS & -- & 117 & 23.56 & 93 & $3 / 15 / 2006$ \\
\hline 17S06W01ADD1 & 331518 & 913956 & USGS & 144 & 182 & 83.58 & 98 & $3 / 15 / 2006$ \\
\hline 17S06W35CAC1 & 331049 & 914136 & USGS & 140 & 179 & 72.62 & 106 & $3 / 15 / 2006$ \\
\hline 18S05W11CCD1 & 330841 & 913538 & NRCS & 75 & 118 & 22.8 & 95 & $3 / 25 / 2006$ \\
\hline 18S05W22DDA1 & 330712 & 913555 & NRCS & 100 & 125 & 21.0 & 104 & $3 / 25 / 2006$ \\
\hline 18S08W01AAB1 & 331015 & 915225 & USGS & 128 & 181 & 86.63 & 94 & $3 / 15 / 2006$ \\
\hline 18S08W28DDD2 & 330625 & 915528 & USGS & 156 & 163.26 & 84.96 & 78 & $5 / 09 / 2006$ \\
\hline 19S04W06BAB2 & 330504 & 913329 & USGS & 98 & 110 & 23.67 & 86 & $3 / 15 / 2006$ \\
\hline 19S05W08ACA1 & 330405 & 913815 & NRCS & -- & 111 & 17.3 & 94 & $3 / 25 / 2006$ \\
\hline 19S05W16ABB1 & 330323 & 913718 & NRCS & 100 & 116 & 24.0 & 92 & $3 / 25 / 2006$ \\
\hline 19S05W22DCD1 & 330139 & 913615 & NRCS & -- & 107 & 23.2 & 84 & $3 / 25 / 2006$ \\
\hline 19S06W07BCC1 & 330404 & 914608 & USGS & -- & 134.7 & 31.04 & 104 & $3 / 15 / 2006$ \\
\hline \multicolumn{9}{|c|}{ Chicot County } \\
\hline 13S03W27AAA1 & 333253 & 912310 & NRCS & -- & 138 & 46 & 92 & $3 / 27 / 2006$ \\
\hline 13S03W34BAA1 & 333110 & 912539 & USGS & 100 & 133 & 40.32 & 93 & $3 / 16 / 2006$ \\
\hline 13S03W34CAA1 & 333136 & 912336 & USGS & 75 & 132 & 37.08 & 95 & $3 / 16 / 2006$ \\
\hline 13S03W35BAC1 & 333154 & 912246 & USGS & 90 & 134 & 39.58 & 94 & $3 / 16 / 2006$ \\
\hline 14S02W09BDD1 & 332859 & 911729 & NRCS & -- & 133 & 29 & 104 & $3 / 27 / 2006$ \\
\hline 14S02W18BBDD1 & 332859 & 912038 & NRCS & -- & 129 & 32 & 97 & $4 / 05 / 2006$ \\
\hline 14S03W07BBD1 & 333011 & 912620 & USGS & 77 & 134 & 26.56 & 107 & $3 / 16 / 2006$ \\
\hline 14S03W32CDB2 & 332613 & 912551 & USGS & 90 & 134 & 34.86 & 99 & $3 / 16 / 2006$ \\
\hline 15S02W20DDC $1 *$ & 332227 & 911920 & USGS & 70 & 126 & 27.91 & 98 & $3 / 16 / 2006$ \\
\hline
\end{tabular}


Appendix 1. Information pertaining to water levels measured in wells completed in the Mississippi River Valley alluvial aquifer in eastern Arkansas, spring 2006.-Continued

[USGS, U.S. Geological Survey; NRCS, Natural Resources Conservation Service; --, no data; NGVD of 1929, National Geodetic Vertical Datum of 1929; Horizontal coordinate information is referenced to the North American Datum of 1983 (NAD 83); *, control wells for duplicate measurements by USGS and NRCS]

\begin{tabular}{|c|c|c|c|c|c|c|c|c|}
\hline Station name & $\begin{array}{c}\text { Latitude } \\
\text { (degrees, } \\
\text { minutes, } \\
\text { seconds) }\end{array}$ & $\begin{array}{c}\text { Longitude } \\
\text { (degrees, } \\
\text { minutes, } \\
\text { seconds) }\end{array}$ & $\begin{array}{c}\text { Source } \\
\text { of } \\
\text { data }\end{array}$ & $\begin{array}{c}\text { Depth } \\
\text { of well } \\
\text { (feet) }\end{array}$ & $\begin{array}{l}\text { Land-surface } \\
\text { datum altitude } \\
\text { (feet above } \\
\text { NGVD of } \\
\text { 1929) }\end{array}$ & $\begin{array}{c}\text { Depth to } \\
\text { water } \\
\text { (feet below } \\
\text { land-surface } \\
\text { datum) }\end{array}$ & $\begin{array}{l}\text { Water-level } \\
\text { altitude } \\
\text { (feet above } \\
\text { NGVD of } \\
\text { 1929) }\end{array}$ & $\begin{array}{c}\text { Date of } \\
\text { measure- } \\
\text { ment }\end{array}$ \\
\hline \multicolumn{9}{|c|}{ Chicot County-Continued } \\
\hline 15S02W20DDC $1 *$ & 332227 & 911920 & NRCS & 70 & 126 & 29 & 97 & $3 / 22 / 2006$ \\
\hline 16S03W11ADC1 & 331920 & 912234 & USGS & -- & 118 & 28.93 & 89 & $3 / 16 / 2006$ \\
\hline 17S01E17CDA1 & 331259 & 910716 & USGS & 110 & 118 & 20.25 & 98 & $3 / 16 / 2006$ \\
\hline 17S01E18ADA1 & 331326 & 910758 & USGS & -- & 121 & 11.04 & 110 & $3 / 16 / 2006$ \\
\hline 17S01W06BCC1 & 331501 & 911505 & USGS & 100 & 115 & 21.03 & 94 & $3 / 16 / 2006$ \\
\hline 17S02W10AAA1 & 331429 & 911712 & USGS & 90 & 114 & 26.35 & 88 & $3 / 16 / 2006$ \\
\hline 17S03W18CBC1 & 331257 & 912736 & NRCS & -- & 117 & 33 & 84 & $3 / 22 / 2006$ \\
\hline 17S03W28DBA1 & 331127 & 912441 & USGS & 95 & 110 & 24.33 & 86 & $3 / 16 / 2006$ \\
\hline 18S01W19DAB1 & 330709 & 911423 & USGS & -- & 110 & 13.07 & 97 & $3 / 15 / 2006$ \\
\hline 18S01W33BAD1 & 330543 & 911245 & NRCS & -- & 116 & 18 & 98 & $3 / 22 / 2006$ \\
\hline 18S03W22ABA2 & 330728 & 912341 & USGS & 85.5 & 103 & 10.56 & 92 & $3 / 15 / 2006$ \\
\hline 19S01W17BCC1 & 330250 & 911406 & USGS & 120 & 106 & 19.76 & 86 & $3 / 15 / 2006$ \\
\hline 19S03W14ABB1 & 330304 & 912251 & USGS & 95 & 111 & 23.74 & 87 & $3 / 15 / 2006$ \\
\hline \multicolumn{9}{|c|}{ Clay County } \\
\hline 18N08E03DAB1 & 361323 & 901153 & USGS & 105 & 257 & 7.89 & 249 & $4 / 18 / 2006$ \\
\hline 18N08E11BAA1 & 361253 & 901117 & NRCS & 100 & 259 & 7 & 252 & $4 / 12 / 2006$ \\
\hline 19N03E24AAA1 & 361655 & 904157 & USGS & -- & 278 & 20.12 & 258 & $4 / 18 / 2006$ \\
\hline 19N04E11DAA1 & 361805 & 903621 & NRCS & -- & 280 & 23 & 257 & $4 / 12 / 2006$ \\
\hline 19N04E19AAA1 & 361654 & 904050 & USGS & -- & 282 & 31.07 & 251 & $4 / 18 / 2006$ \\
\hline 19N04E19BAA1 & 361649 & 904125 & NRCS & 100 & 279 & 22 & 257 & $4 / 12 / 2006$ \\
\hline 19N05E15BBD1 & 361716 & 903152 & NRCS & 110 & 289 & 34 & 255 & $4 / 12 / 2006$ \\
\hline 19N06E18DBC1 & 361642 & 902815 & NRCS & -- & 297 & 37 & 260 & $4 / 13 / 2006$ \\
\hline 19N07E25BCB1 & 361519 & 901700 & NRCS & -- & 268 & 18 & 250 & $4 / 12 / 2006$ \\
\hline 19N08E08DCA1 & 361729 & 901402 & NRCS & -- & 270 & 7 & 263 & $4 / 12 / 2006$ \\
\hline 19N09E19CDC1 & 361539 & 900908 & NRCS & -- & 265 & 8 & 257 & $4 / 12 / 2006$ \\
\hline 20N03E25BAA1 & 362112 & 904225 & NRCS & 100 & 288 & 22 & 266 & $4 / 13 / 2006$ \\
\hline 20N04E03ADA1 & 362425 & 903725 & NRCS & -- & 290 & 16 & 274 & $4 / 13 / 2006$ \\
\hline 20N04E06BB1 & 362444 & 904131 & USGS & 110 & 290 & 19.97 & 270 & 4/18/2006 \\
\hline 20N05E22CAD1 & 362118 & 903132 & NRCS & -- & 290 & 31 & 259 & $4 / 12 / 2006$ \\
\hline 20N05E30CAC1 & 362003 & 903454 & NRCS & -- & 283 & 18 & 265 & $4 / 12 / 2006$ \\
\hline 20N05E34DBA1 & 361939 & 903117 & USGS & 110 & 285 & 29.31 & 256 & $4 / 18 / 2006$ \\
\hline 20N06E09BBA1 & 362327 & 902620 & NRCS & -- & 290 & 22 & 268 & $4 / 12 / 2006$ \\
\hline 20N06E28CCD1 & 362005 & 902630 & NRCS & -- & 290 & 29 & 261 & $4 / 12 / 2006$ \\
\hline 20N08E22BDC1 & 362111 & 901220 & NRCS & -- & 275 & 9 & 266 & $4 / 12 / 2006$ \\
\hline
\end{tabular}


Appendix 1. Information pertaining to water levels measured in wells completed in the Mississippi River Valley alluvial aquifer in eastern Arkansas, spring 2006.-Continued

[USGS, U.S. Geological Survey; NRCS, Natural Resources Conservation Service; --, no data; NGVD of 1929, National Geodetic Vertical Datum of 1929; Horizontal coordinate information is referenced to the North American Datum of 1983 (NAD 83); *, control wells for duplicate measurements by USGS and NRCS]

\begin{tabular}{|c|c|c|c|c|c|c|c|c|}
\hline Station name & $\begin{array}{c}\text { Latitude } \\
\text { (degrees, } \\
\text { minutes, } \\
\text { seconds) }\end{array}$ & $\begin{array}{c}\text { Longitude } \\
\text { (degrees, } \\
\text { minutes, } \\
\text { seconds) }\end{array}$ & $\begin{array}{c}\text { Source } \\
\text { of } \\
\text { data }\end{array}$ & $\begin{array}{c}\text { Depth } \\
\text { of well } \\
\text { (feet) }\end{array}$ & $\begin{array}{l}\text { Land-surface } \\
\text { datum altitude } \\
\text { (feet above } \\
\text { NGVD of } \\
\text { 1929) }\end{array}$ & $\begin{array}{c}\text { Depth to } \\
\text { water } \\
\text { (feet below } \\
\text { land-surface } \\
\text { datum) }\end{array}$ & $\begin{array}{l}\text { Water-level } \\
\text { altitude } \\
\text { (feet above } \\
\text { NGVD of } \\
\text { 1929) }\end{array}$ & $\begin{array}{c}\text { Date of } \\
\text { measure- } \\
\text { ment }\end{array}$ \\
\hline \multicolumn{9}{|c|}{ Clay County-Continued } \\
\hline 20N08E24DDA1 & 362057 & 900934 & USGS & 110 & 276 & 9.75 & 266 & $4 / 18 / 2006$ \\
\hline 20N09E09ABC1 & 362306 & 900642 & NRCS & -- & 279 & 8 & 271 & $4 / 12 / 2006$ \\
\hline 20N09E33DDC1 & 361904 & 900628 & NRCS & -- & 270 & 7 & 263 & $4 / 12 / 2006$ \\
\hline 21N03E15CBC1 & 362738 & 904453 & NRCS & 90 & 292 & 11 & 281 & $4 / 13 / 2006$ \\
\hline 21N03E36CDD1 & 362450 & 904214 & NRCS & -- & 290 & 19 & 271 & $4 / 13 / 2006$ \\
\hline 21N04E09DBC1 & 362828 & 903853 & NRCS & -- & 291 & 13 & 278 & $4 / 13 / 2006$ \\
\hline 21N05E17ABB1 & 362755 & 903329 & USGS & 105 & 298 & 23.23 & 275 & $4 / 17 / 2006$ \\
\hline 21N05E22BAB1 & 362704 & 903132 & NRCS & 105 & 288 & 7 & 281 & $4 / 13 / 2006$ \\
\hline 21N06E11BBB1 & 362839 & 902421 & NRCS & 100 & 296 & 15 & 281 & $4 / 13 / 2006$ \\
\hline 21N06E28BB1 & 362605 & 902608 & USGS & 130 & 292.5 & 19.19 & 273 & $4 / 17 / 2006$ \\
\hline 21N07E01DDC1 & 362835 & 901607 & NRCS & 90 & 303 & 26 & 277 & $4 / 13 / 2006$ \\
\hline 21N08E03CDB1 & 362848 & 901217 & NRCS & -- & 308 & 19 & 289 & $4 / 13 / 2006$ \\
\hline 21N08E18CCC1 & 362651 & 901550 & USGS & 110 & 324 & 38.64 & 285 & $4 / 17 / 2006$ \\
\hline 21N08E36ABB1 & 362502 & 900958 & USGS & 90 & 283 & 4.05 & 279 & $4 / 18 / 2006$ \\
\hline 21N09E31BDA1 & 362447 & 900851 & NRCS & 100 & 284 & 7 & 277 & $4 / 12 / 2006$ \\
\hline \multicolumn{9}{|c|}{ Craighead County } \\
\hline 13N01E03AAA1 & 354739 & 905753 & NRCS & 135 & 240 & 54.7 & 185 & $3 / 01 / 2006$ \\
\hline 13N01E21CAB & 354434 & 905945 & NRCS & 120 & 240 & 62.0 & 178 & $3 / 01 / 2006$ \\
\hline 13N01E23CAB1 & 354430 & 905736 & NRCS & 118 & 245 & 68.5 & 177 & $3 / 01 / 2006$ \\
\hline 13N01E23DAA1 & 354435 & 905652 & USGS & 118 & 242 & 71.02 & 171 & $4 / 19 / 2006$ \\
\hline 13N02E02AAB1 & 354731 & 905032 & NRCS & 130 & 251 & 92.2 & 159 & $3 / 01 / 2006$ \\
\hline 13N02E03AAA1 & 354733 & 905129 & NRCS & 105 & 250 & 86.9 & 163 & $3 / 01 / 2006$ \\
\hline 13N03E23CDA1 & 354419 & 904434 & NRCS & 135 & 249 & 79.6 & 169 & $3 / 02 / 2006$ \\
\hline 13N03E28CDB1 & 354322 & 904652 & NRCS & 121 & 250 & 109.0 & 141 & $3 / 02 / 2006$ \\
\hline 13N03E29AAA1 & 354403 & 904713 & USGS & 122 & 251 & 103.76 & 147 & $4 / 19 / 2006$ \\
\hline 13N03E35AAA1 & 354308 & 904401 & NRCS & 150 & 249 & 94.0 & 155 & $3 / 02 / 2006$ \\
\hline 13N04E12ABB1 & 354635 & 903656 & USGS & 110 & 231 & 23.82 & 207 & $4 / 19 / 2006$ \\
\hline 13N04E15DBA1 & 354521 & 903857 & NRCS & 130 & 230 & 26.6 & 203 & $3 / 02 / 2006$ \\
\hline 13N04E26BCC1 & 354340 & 903829 & NRCS & 100 & 225 & 26.5 & 199 & $3 / 02 / 2006$ \\
\hline 13N05E02CCC1 & 354648 & 903202 & NRCS & 120 & 230 & 12.9 & 217 & $3 / 02 / 2006$ \\
\hline 13N05E06DCC1 & 354637 & 903547 & NRCS & 110 & 229 & 20.0 & 209 & $3 / 02 / 2006$ \\
\hline 13N05E22BAD1 & 354449 & 903243 & USGS & -- & 226 & 15.39 & 211 & $4 / 19 / 2006$ \\
\hline 13N05E24BAC1 & 354451 & 903045 & NRCS & 120 & 225 & 12.2 & 213 & $3 / 02 / 2006$ \\
\hline 13N06E21AAA1 & 354450 & 902701 & NRCS & 150 & 222 & 10.0 & 212 & $3 / 02 / 2006$ \\
\hline
\end{tabular}


Appendix 1. Information pertaining to water levels measured in wells completed in the Mississippi River Valley alluvial aquifer in eastern Arkansas, spring 2006.-Continued

[USGS, U.S. Geological Survey; NRCS, Natural Resources Conservation Service; --, no data; NGVD of 1929, National Geodetic Vertical Datum of 1929; Horizontal coordinate information is referenced to the North American Datum of 1983 (NAD 83); *, control wells for duplicate measurements by USGS and NRCS]

\begin{tabular}{|c|c|c|c|c|c|c|c|c|}
\hline Station name & $\begin{array}{c}\text { Latitude } \\
\text { (degrees, } \\
\text { minutes, } \\
\text { seconds) }\end{array}$ & $\begin{array}{l}\text { Longitude } \\
\text { (degrees, } \\
\text { minutes, } \\
\text { seconds) }\end{array}$ & $\begin{array}{c}\text { Source } \\
\text { of } \\
\text { data }\end{array}$ & $\begin{array}{c}\text { Depth } \\
\text { of well } \\
\text { (feet) }\end{array}$ & $\begin{array}{l}\text { Land-surface } \\
\text { datum altitude } \\
\text { (feet above } \\
\text { NGVD of } \\
\text { 1929) }\end{array}$ & $\begin{array}{c}\text { Depth to } \\
\text { water } \\
\text { (feet below } \\
\text { land-surface } \\
\text { datum) }\end{array}$ & $\begin{array}{c}\text { Water-level } \\
\text { altitude } \\
\text { (feet above } \\
\text { NGVD of } \\
\text { 1929) }\end{array}$ & $\begin{array}{c}\text { Date of } \\
\text { measure } \\
\text { ment }\end{array}$ \\
\hline \multicolumn{9}{|c|}{ Craighead County—Continued } \\
\hline 13N07E02CAB1 & 354642 & 901901 & NRCS & 120 & 226 & 5.0 & 221 & $3 / 02 / 2006$ \\
\hline 13N07E05ABB1 & 354716 & 902158 & NRCS & 100 & 225 & 5.5 & 220 & $3 / 02 / 2006$ \\
\hline 13N07E20BBA1 & 354440 & 902216 & USGS & 22.3 & 223.2 & 5.42 & 218 & 4/19/2006 \\
\hline 13N07E35BCD1 & 354233 & 901837 & NRCS & 120 & 221 & 12.3 & 209 & $3 / 02 / 2006$ \\
\hline 14N01E03ACB1 & 355246 & 905816 & NRCS & 96 & 249 & 50.9 & 198 & $3 / 01 / 2006$ \\
\hline 14N01E10BAB1 & 355204 & 905828 & NRCS & 96 & 246 & 51.1 & 195 & $3 / 01 / 2006$ \\
\hline 14N01E31DCA1 & 354817 & 910121 & NRCS & 126 & 251 & 59.1 & 192 & $3 / 01 / 2006$ \\
\hline 14N02E18BDD1 & 355041 & 905419 & USGS & 120 & 242 & 51.76 & 190 & 4/19/2006 \\
\hline 14N02E22AAA1 & 355007 & 905129 & NRCS & 132 & 255 & 74.8 & 180 & $3 / 01 / 2006$ \\
\hline 14N05E25ABB1 & 354921 & 903025 & USGS & -- & 238 & 21.54 & 216 & 4/19/2006 \\
\hline 14N06E06BAA1 & 355234 & 902934 & NRCS & 120 & 240 & 22.4 & 218 & $3 / 02 / 2006$ \\
\hline 14N06E27AAB1 & 354911 & 902559 & USGS & 30.3 & 225.93 & 3.1 & 223 & 4/19/2006 \\
\hline 14N07E14DDC1 & 354956 & 901831 & NRCS & 120 & 230 & 13.5 & 217 & $3 / 02 / 2006$ \\
\hline 14N07E26DBB1 & 354834 & 901843 & USGS & 100 & 228 & 9.76 & 218 & $4 / 19 / 2006$ \\
\hline 15N02E12DCB1 & 355626 & 904930 & NRCS & 120 & 250 & 34.5 & 216 & $3 / 01 / 2006$ \\
\hline 15N03E19ADA1 & 355502 & 904802 & USGS & 116 & 262 & 49.16 & 213 & 4/19/2006 \\
\hline 15N03E31ADA1 & 355314 & 904807 & NRCS & 150 & 270 & 63.9 & 206 & $3 / 01 / 2006$ \\
\hline 15N05E22BAB1 & 355513 & 903241 & NRCS & 197 & 260 & 34.8 & 225 & $3 / 02 / 2006$ \\
\hline 15N06E04BAD1 & 355744 & 902706 & NRCS & 104 & 239 & 15.5 & 224 & $3 / 02 / 2006$ \\
\hline 15N06E20DDD1 & 355426 & 902739 & USGS & -- & 234 & 11.22 & 223 & 4/19/2006 \\
\hline 15N07E10DAB1 & 355622 & 901934 & NRCS & 106 & 235 & 9.8 & 225 & $3 / 02 / 2006$ \\
\hline 15N07E10DBA1 & 355628 & 901944 & USGS & 120 & 236 & 9.44 & 227 & 4/19/2006 \\
\hline 15N07E35DCB1 & 355241 & 901831 & NRCS & 120 & 231 & 14.6 & 216 & $3 / 02 / 2006$ \\
\hline \multicolumn{9}{|c|}{ Crittenden County } \\
\hline 04N07E21AAD1 & 345644 & 902121 & USGS & 82.1 & 202 & 13.49 & 189 & $3 / 30 / 2006$ \\
\hline 05N07E08BDC1 & 350407 & 902234 & NRCS & 110 & 204 & 22.8 & 181 & $4 / 27 / 2006$ \\
\hline 05N07E28CBA1 & 350121 & 902140 & USGS & -- & 201 & 19.43 & 182 & $3 / 30 / 2006$ \\
\hline 05N07E34BAB1 & 350059 & 902030 & USGS & 100 & 203 & 19.46 & 184 & $3 / 30 / 2006$ \\
\hline 05N07E34CDD1 & 350010 & 902028 & NRCS & 110 & 205 & 19.0 & 186 & $4 / 26 / 2006$ \\
\hline 05N08E11CCD2 & 350345 & 901308 & USGS & 63 & 211 & 28.32 & 183 & $3 / 30 / 2006$ \\
\hline 06N07E13BAA1 & 350850 & 901808 & USGS & 130 & 205 & 20.28 & 185 & $3 / 30 / 2006$ \\
\hline 06N07E14ABA1 & 350848 & 901858 & NRCS & 110 & 211 & 20.4 & 191 & $4 / 26 / 2006$ \\
\hline 07N06E29CBC1 & 351152 & 902914 & NRCS & 120 & 210 & 39.1 & 171 & $4 / 26 / 2006$ \\
\hline 07N07E31CCC1 & 351042 & 902359 & USGS & 110 & 207 & 34.92 & 172 & $3 / 30 / 2006$ \\
\hline
\end{tabular}


Appendix 1. Information pertaining to water levels measured in wells completed in the Mississippi River Valley alluvial aquifer in eastern Arkansas, spring 2006.-Continued

[USGS, U.S. Geological Survey; NRCS, Natural Resources Conservation Service; --, no data; NGVD of 1929, National Geodetic Vertical Datum of 1929; Horizontal coordinate information is referenced to the North American Datum of 1983 (NAD 83); *, control wells for duplicate measurements by USGS and NRCS]

\begin{tabular}{|c|c|c|c|c|c|c|c|c|}
\hline Station name & $\begin{array}{c}\text { Latitude } \\
\text { (degrees, } \\
\text { minutes, } \\
\text { seconds) }\end{array}$ & $\begin{array}{c}\text { Longitude } \\
\text { (degrees, } \\
\text { minutes, } \\
\text { seconds) }\end{array}$ & $\begin{array}{c}\text { Source } \\
\text { of } \\
\text { data }\end{array}$ & $\begin{array}{c}\text { Depth } \\
\text { of well } \\
\text { (feet) }\end{array}$ & $\begin{array}{l}\text { Land-surface } \\
\text { datum altitude } \\
\text { (feet above } \\
\text { NGVD of } \\
\text { 1929) }\end{array}$ & $\begin{array}{c}\text { Depth to } \\
\text { water } \\
\text { (feet below } \\
\text { land-surface } \\
\text { datum) }\end{array}$ & $\begin{array}{l}\text { Water-level } \\
\text { altitude } \\
\text { (feet above } \\
\text { NGVD of } \\
\text { 1929) }\end{array}$ & $\begin{array}{c}\text { Date of } \\
\text { measure- } \\
\text { ment }\end{array}$ \\
\hline \multicolumn{9}{|c|}{ Crittenden County-Continued } \\
\hline 07N08E04BBD1 & 351538 & 901505 & NRCS & 120 & 224 & 19.1 & 205 & $4 / 25 / 2006$ \\
\hline 07N09E05CDD1 & 351453 & 900934 & USGS & 120 & 214 & 16.94 & 197 & $3 / 30 / 2006$ \\
\hline 08N06E01DCC1 & 352021 & 902408 & NRCS & 120 & 215 & 32.0 & 183 & $4 / 25 / 2006$ \\
\hline 08N06E06DDB1 & 352030 & 902920 & NRCS & 120 & 214 & 30.2 & 184 & $4 / 25 / 2006$ \\
\hline 08N07E13CCC2 & 351828 & 901812 & USGS & 100 & 221 & 29.2 & 192 & $3 / 30 / 2006$ \\
\hline 08N07E14DAA2 & 351854 & 901833 & USGS & -- & 219 & 29.98 & 189 & $3 / 30 / 2006$ \\
\hline 08N07E32DAA1 & 351618 & 902146 & NRCS & 110 & 215 & 29.0 & 186 & $4 / 25 / 2006$ \\
\hline 08N08E06ABB1 & 352103 & 901644 & NRCS & 110 & 223 & 29.0 & 194 & $4 / 25 / 2006$ \\
\hline 09N07E02CDB1 & 352537 & 901905 & NRCS & 130 & 225 & 31.7 & 193 & $4 / 25 / 2006$ \\
\hline 09N07E10DDA1 & 352448 & 901925 & USGS & 60 & 221 & 27.85 & 193 & $3 / 30 / 2006$ \\
\hline 09N07E31BAB $1 *$ & 352160 & 902327 & USGS & 110 & 221 & 32.40 & 189 & $3 / 30 / 2006$ \\
\hline 09N07E31BAB $1 *$ & 352160 & 902327 & NRCS & 110 & 221 & 31.8 & 189 & $4 / 25 / 2006$ \\
\hline 09N08E04CDC1 & 352527 & 901444 & NRCS & 120 & 225 & 24.1 & 201 & $4 / 25 / 2006$ \\
\hline \multicolumn{9}{|c|}{ Cross County } \\
\hline 06N02E04DCD1 & 350953 & 905322 & NRCS & -- & 217 & 79 & 138 & $4 / 27 / 2006$ \\
\hline 06N02E11BDB1 & 350934 & 905132 & NRCS & -- & 220 & 62 & 158 & $4 / 26 / 2006$ \\
\hline 06N02E12AAA1 & 350934 & 904952 & NRCS & -- & 235 & 78 & 157 & $4 / 26 / 2006$ \\
\hline 06N04E01BBB1 & 351044 & 903739 & NRCS & -- & 205 & 37 & 168 & $5 / 04 / 2006$ \\
\hline 06N05E03AAD1 & 351028 & 903218 & NRCS & 100 & 208 & 39 & 169 & $5 / 04 / 2006$ \\
\hline 06N05E05AAA1 & 351042 & 903432 & NRCS & 130 & 205 & 30 & 175 & $5 / 04 / 2006$ \\
\hline 07N01E05CDA1 & 351518 & 910049 & USGS & 140 & 217 & 73.64 & 143 & $3 / 29 / 2006$ \\
\hline 07N01E05DCA1 & 351514 & 910033 & NRCS & 160 & 215 & 73 & 142 & $4 / 26 / 2006$ \\
\hline 07N01E06AAB1 & 351556 & 910132 & NRCS & -- & 218 & 72 & 146 & $4 / 24 / 2006$ \\
\hline 07N01E06CAA1 & 351530 & 910154 & NRCS & -- & 220 & 73 & 147 & $4 / 24 / 2006$ \\
\hline 07N01E11AAA1 & 351501 & 905705 & USGS & 120 & 217 & 76.58 & 140 & $3 / 29 / 2006$ \\
\hline 07N01E22BBB1 & 351321 & 905913 & NRCS & 100 & 215 & 68 & 147 & $4 / 24 / 2006$ \\
\hline 07N01E33BBA1 & 351134 & 910010 & NRCS & -- & 215 & 68 & 147 & $4 / 24 / 2006$ \\
\hline 07N02E02BBB1 & 351601 & 905144 & NRCS & -- & 227 & 75 & 152 & $4 / 26 / 2006$ \\
\hline 07N02E02DCC1 & 351512 & 905112 & NRCS & -- & 224 & 80 & 144 & $4 / 27 / 2006$ \\
\hline 07N02E10ABB1 & 351504 & 905217 & NRCS & -- & 230 & 84 & 146 & $4 / 27 / 2006$ \\
\hline 07N02E12BBC1 & 351447 & 905040 & NRCS & 100 & 225 & 80 & 145 & $4 / 27 / 2006$ \\
\hline 07N02E15DAA1 & 351330 & 905149 & NRCS & -- & 218 & 79 & 139 & $4 / 27 / 2006$ \\
\hline 07N02E29AAA1 & 351223 & 905404 & NRCS & -- & 220 & 69 & 151 & $4 / 26 / 2006$ \\
\hline 07N02E29CCC1 & 351142 & 905152 & NRCS & -- & 220 & 70 & 150 & $4 / 26 / 2006$ \\
\hline
\end{tabular}


Appendix 1. Information pertaining to water levels measured in wells completed in the Mississippi River Valley alluvial aquifer in eastern Arkansas, spring 2006. - Continued

[USGS, U.S. Geological Survey; NRCS, Natural Resources Conservation Service; --, no data; NGVD of 1929, National Geodetic Vertical Datum of 1929; Horizontal coordinate information is referenced to the North American Datum of 1983 (NAD 83); *, control wells for duplicate measurements by USGS and NRCS]

\begin{tabular}{|c|c|c|c|c|c|c|c|c|}
\hline Station name & $\begin{array}{c}\text { Latitude } \\
\text { (degrees, } \\
\text { minutes, } \\
\text { seconds) }\end{array}$ & $\begin{array}{l}\text { Longitude } \\
\text { (degrees, } \\
\text { minutes, } \\
\text { seconds) }\end{array}$ & $\begin{array}{c}\text { Source } \\
\text { of } \\
\text { data }\end{array}$ & $\begin{array}{l}\text { Depth } \\
\text { of well } \\
\text { (feet) }\end{array}$ & $\begin{array}{l}\text { Land-surface } \\
\text { datum altitude } \\
\text { (feet above } \\
\text { NGVD of } \\
\text { 1929) }\end{array}$ & $\begin{array}{c}\text { Depth to } \\
\text { water } \\
\text { (feet below } \\
\text { land-surface } \\
\text { datum) }\end{array}$ & $\begin{array}{c}\text { Water-level } \\
\text { altitude } \\
\text { (feet above } \\
\text { NGVD of } \\
\text { 1929) }\end{array}$ & $\begin{array}{c}\text { Date of } \\
\text { measure- } \\
\text { ment }\end{array}$ \\
\hline \multicolumn{9}{|c|}{ Cross County—Continued } \\
\hline 07N02E29DDC1* & 351138 & 905409 & USGS & 100 & 220 & 72.26 & 148 & 3/29/2006 \\
\hline 07N02E29DDC1* & 351138 & 905409 & NRCS & 100 & 220 & 70 & 150 & $4 / 24 / 2006$ \\
\hline 07N03E05ADA1 & 351549 & 904739 & USGS & 160 & 254 & 124.05 & 130 & $3 / 29 / 2006$ \\
\hline 07N03E32DCC1 & 351045 & 904810 & USGS & -- & 251 & 96.38 & 155 & $3 / 29 / 2006$ \\
\hline 07N04E03BDA1 & 351546 & 903925 & NRCS & -- & 205 & 29 & 176 & $5 / 01 / 2006$ \\
\hline 07N04E04DBB1 & 351534 & 904021 & NRCS & -- & 201 & 30 & 171 & $5 / 01 / 2006$ \\
\hline 07N04E07AAA1 & 351510 & 904207 & NRCS & -- & 223 & 45 & 178 & $5 / 01 / 2006$ \\
\hline 07N04E27BDA1 & 351220 & 903926 & NRCS & -- & 203 & 27 & 176 & $5 / 03 / 2006$ \\
\hline 07N05E02AAB1 & 351600 & 903103 & NRCS & -- & 210 & 41 & 169 & $5 / 04 / 2006$ \\
\hline 07N05E16ACA1 & 351358 & 903352 & NRCS & -- & 210 & 33 & 177 & $5 / 04 / 2006$ \\
\hline 07N05E19CCC1 & 351238 & 903645 & USGS & -- & 207 & 37.27 & 170 & $3 / 29 / 2006$ \\
\hline 07N05E24CCC1 & 351232 & 903121 & NRCS & 110 & 205 & 34.9 & 170 & $4 / 26 / 2006$ \\
\hline 07N05E25ABA1 & 351229 & 903045 & USGS & 140 & 205 & 36.71 & 168 & $3 / 29 / 2006$ \\
\hline 07N05E32DDC1 & 351053 & 903500 & NRCS & -- & 205 & 38 & 167 & $5 / 04 / 2006$ \\
\hline 08N01E02CDD1 & 352023 & 905736 & NRCS & -- & 226 & 84 & 142 & 4/27/2006 \\
\hline 08N01E05DBB1 & 352044 & 910038 & NRCS & -- & 223 & 76 & 147 & $4 / 24 / 2006$ \\
\hline 08N01E16DBB1 & 351855 & 905933 & NRCS & 140 & 225 & 84 & 141 & 4/27/2006 \\
\hline 08N01E17CAD1 & 351852 & 910046 & NRCS & -- & 220 & 74 & 146 & $4 / 24 / 2006$ \\
\hline 08N01E32CBB1 & 351622 & 910048 & NRCS & -- & 221 & 71 & 150 & $4 / 24 / 2006$ \\
\hline 08N02E12DCC1 & 351938 & 905002 & NRCS & -- & 230 & 88 & 142 & $4 / 27 / 2006$ \\
\hline 08N02E17AAA1 & 351923 & 905354 & NRCS & -- & 225 & 85 & 140 & $4 / 27 / 2006$ \\
\hline 08N02E29CBA1 & 351715 & 905438 & NRCS & -- & 225 & 80 & 145 & $4 / 27 / 2006$ \\
\hline 08N03E15BBB1 & 351942 & 904620 & NRCS & -- & 265 & 112 & 153 & $4 / 27 / 2006$ \\
\hline 08N04E34CCD1 & 351605 & 903945 & NRCS & -- & 205 & 28 & 177 & $5 / 01 / 2006$ \\
\hline 08N05E17CAA1 & 351904 & 903508 & NRCS & -- & 211 & 30 & 181 & $5 / 04 / 2006$ \\
\hline 08N05E32ADD1 & 351632 & 903440 & USGS & -- & 204 & 29.57 & 174 & $3 / 29 / 2006$ \\
\hline 09N01E04ACD1 & 352608 & 905914 & NRCS & 140 & 225 & 88 & 137 & $4 / 24 / 2006$ \\
\hline 09N01E33BBA1 & 352204 & 905959 & NRCS & 120 & 225 & 79 & 146 & $4 / 26 / 2006$ \\
\hline 09N01E33BBA2 & 352203 & 910001 & USGS & -- & 225 & 81.10 & 144 & $3 / 29 / 2006$ \\
\hline 09N01E36AAB1 & 352155 & 905605 & NRCS & 160 & 225 & 85 & 140 & $4 / 27 / 2006$ \\
\hline 09N01E36BBB1 & 352200 & 905650 & NRCS & 90 & 226 & 85 & 141 & $4 / 27 / 2006$ \\
\hline 09N02E17AAB1 & 352438 & 905359 & NRCS & 100 & 232 & 94 & 138 & $4 / 27 / 2006$ \\
\hline 09N02E20AAA1 & 352402 & 905342 & NRCS & 120 & 231 & 94 & 137 & 4/27/2006 \\
\hline 09N02E30CBB1 & 352243 & 905551 & NRCS & -- & 225 & 87 & 138 & $4 / 27 / 2006$ \\
\hline
\end{tabular}


Appendix 1. Information pertaining to water levels measured in wells completed in the Mississippi River Valley alluvial aquifer in eastern Arkansas, spring 2006.-Continued

[USGS, U.S. Geological Survey; NRCS, Natural Resources Conservation Service; --, no data; NGVD of 1929, National Geodetic Vertical Datum of 1929; Horizontal coordinate information is referenced to the North American Datum of 1983 (NAD 83); *, control wells for duplicate measurements by USGS and NRCS]

\begin{tabular}{|c|c|c|c|c|c|c|c|c|}
\hline Station name & $\begin{array}{c}\text { Latitude } \\
\text { (degrees, } \\
\text { minutes, } \\
\text { seconds) }\end{array}$ & $\begin{array}{c}\text { Longitude } \\
\text { (degrees, } \\
\text { minutes, } \\
\text { seconds) }\end{array}$ & $\begin{array}{c}\text { Source } \\
\text { of } \\
\text { data }\end{array}$ & $\begin{array}{c}\text { Depth } \\
\text { of well } \\
\text { (feet) }\end{array}$ & $\begin{array}{l}\text { Land-surface } \\
\text { datum altitude } \\
\text { (feet above } \\
\text { NGVD of } \\
\text { 1929) }\end{array}$ & $\begin{array}{c}\text { Depth to } \\
\text { water } \\
\text { (feet below } \\
\text { land-surface } \\
\text { datum) }\end{array}$ & $\begin{array}{l}\text { Water-level } \\
\text { altitude } \\
\text { (feet above } \\
\text { NGVD of } \\
\text { 1929) }\end{array}$ & $\begin{array}{c}\text { Date of } \\
\text { measure- } \\
\text { ment }\end{array}$ \\
\hline \multicolumn{9}{|c|}{ Cross County-Continued } \\
\hline 09N02E32BBB1 & 352213 & 905444 & NRCS & -- & 226 & 94 & 132 & $4 / 28 / 2006$ \\
\hline 09N03E03ACA1 & 352630 & 904529 & NRCS & -- & 250 & 106 & 144 & $4 / 27 / 2006$ \\
\hline 09N03E17CDD1 & 352422 & 904753 & NRCS & -- & 245 & 99 & 146 & $4 / 27 / 2006$ \\
\hline 09N03E17DDC1 & 352409 & 904726 & USGS & 160 & 251 & 105.65 & 145 & $3 / 29 / 2006$ \\
\hline 09N03E18ABC1 & 352448 & 904851 & NRCS & -- & 235 & 98 & 137 & $4 / 27 / 2006$ \\
\hline 09N04E01AAC1 & 352622 & 903648 & NRCS & -- & 205 & 15 & 190 & $5 / 04 / 2006$ \\
\hline 09N04E03DBB1 & 352614 & 903918 & NRCS & 120 & 215 & 25 & 190 & $5 / 04 / 2006$ \\
\hline 09N04E33ABC1 & 352204 & 904020 & NRCS & -- & 216 & 37 & 179 & $5 / 04 / 2006$ \\
\hline 09N05E10ACC1 & 352511 & 903249 & NRCS & 120 & 210 & 23 & 187 & $5 / 04 / 2006$ \\
\hline 09N05E15CBC1 & 352413 & 903302 & NRCS & -- & 210 & 23 & 187 & $5 / 04 / 2006$ \\
\hline 09N05E32BCB1 & 352151 & 903525 & NRCS & -- & 206 & 30 & 176 & $5 / 04 / 2006$ \\
\hline 09N05E32BDB1 & 352151 & 903512 & USGS & -- & 210 & 30.63 & 179 & $3 / 29 / 2006$ \\
\hline \multicolumn{9}{|c|}{ Desha County } \\
\hline 07S01E19ABA1 & 340428 & 910303 & NRCS & 120 & 154 & 21 & 133 & $4 / 12 / 2006$ \\
\hline 08S03W33ABD1 & 335803 & 912338 & USGS & 60 & 165.04 & 7.24 & 158 & $3 / 17 / 2006$ \\
\hline 09S01W08BDA1 & 335608 & 911234 & NRCS & -- & 156 & 28 & 128 & $4 / 07 / 2006$ \\
\hline 09S01W15CBB1 & 335501 & 911055 & NRCS & -- & 152 & 36 & 116 & $4 / 07 / 2006$ \\
\hline 09S02W26DDC1 & 335257 & 911530 & USGS & 94 & 149.27 & 29.33 & 120 & $5 / 09 / 2006$ \\
\hline 09S03W05BAC1 & 335704 & 912506 & NRCS & -- & 161 & 42 & 119 & $4 / 07 / 2006$ \\
\hline 09S03W13BAB1 & 335500 & 911922 & NRCS & -- & 156 & 32 & 124 & $4 / 07 / 2006$ \\
\hline 09S03W17DCB1 & 335448 & 912457 & USGS & 126 & 155.08 & 32.95 & 122 & $3 / 17 / 2006$ \\
\hline 09S04W06BCA1 & 335756 & 913243 & USGS & -- & 161 & 34.92 & 126 & $3 / 17 / 2006$ \\
\hline 10S01W23CDA1 & 335305 & 911032 & NRCS & -- & 151 & 26 & 125 & $4 / 07 / 2006$ \\
\hline 10S02W11ADD1 & 335045 & 911517 & NRCS & -- & 146 & 28 & 118 & $4 / 07 / 2006$ \\
\hline 10S02W24DBC1 & 334850 & 911453 & USGS & 70 & 143 & 25.53 & 117 & $3 / 17 / 2006$ \\
\hline 10S03W26CAA1 & 334806 & 912145 & USGS & 96 & 155 & 44.80 & 110 & $3 / 17 / 2006$ \\
\hline 10S04W03ABB1 & 335208 & 912931 & USGS & 100 & 165 & 33.71 & 131 & $5 / 18 / 2006$ \\
\hline 10S04W03BAB1 & 335209 & 912948 & USGS & 100 & 166 & 35.09 & 131 & $5 / 18 / 2006$ \\
\hline 10S04W11DDA1 & 335031 & 912802 & USGS & 100 & 155 & 31.50 & 124 & $5 / 18 / 2006$ \\
\hline 10S04W12DBB1 & 335102 & 912729 & USGS & 99 & 152 & 28.95 & 123 & $5 / 18 / 2006$ \\
\hline 11S02W15ADD1 & 334446 & 911635 & NRCS & -- & 144 & 34 & 110 & $4 / 07 / 2006$ \\
\hline 11S03W16CBA1 & 334439 & 912433 & NRCS & -- & 155 & 31 & 124 & $4 / 07 / 2006$ \\
\hline 11S03W31BBA1 & 334228 & 912651 & USGS & -- & 148 & 35.14 & 113 & $3 / 17 / 2006$ \\
\hline 12S01W33BAA1 & 333718 & 911205 & USGS & 95 & 135 & 23.92 & 111 & $3 / 17 / 2006$ \\
\hline
\end{tabular}


Appendix 1. Information pertaining to water levels measured in wells completed in the Mississippi River Valley alluvial aquifer in eastern Arkansas, spring 2006.-Continued

[USGS, U.S. Geological Survey; NRCS, Natural Resources Conservation Service; --, no data; NGVD of 1929, National Geodetic Vertical Datum of 1929; Horizontal coordinate information is referenced to the North American Datum of 1983 (NAD 83); *, control wells for duplicate measurements by USGS and NRCS]

\begin{tabular}{|c|c|c|c|c|c|c|c|c|}
\hline Station name & $\begin{array}{c}\text { Latitude } \\
\text { (degrees, } \\
\text { minutes, } \\
\text { seconds) }\end{array}$ & $\begin{array}{c}\text { Longitude } \\
\text { (degrees, } \\
\text { minutes, } \\
\text { seconds) }\end{array}$ & $\begin{array}{c}\text { Source } \\
\text { of } \\
\text { data }\end{array}$ & $\begin{array}{c}\text { Depth } \\
\text { of well } \\
\text { (feet) }\end{array}$ & $\begin{array}{l}\text { Land-surface } \\
\text { datum altitude } \\
\text { (feet above } \\
\text { NGVD of } \\
\text { 1929) }\end{array}$ & $\begin{array}{c}\text { Depth to } \\
\text { water } \\
\text { (feet below } \\
\text { land-surface } \\
\text { datum) }\end{array}$ & $\begin{array}{l}\text { Water-level } \\
\text { altitude } \\
\text { (feet above } \\
\text { NGVD of } \\
\text { 1929) }\end{array}$ & $\begin{array}{c}\text { Date of } \\
\text { measure- } \\
\text { ment }\end{array}$ \\
\hline \multicolumn{9}{|c|}{ Desha County-Continued } \\
\hline 13S02W17ADA1 & 333421 & 911858 & NRCS & -- & 138 & 45 & 93 & $4 / 07 / 2006$ \\
\hline 13S02W27CAC1 & 333224 & 911735 & USGS & 120 & 133 & 30.83 & 102 & $3 / 17 / 2006$ \\
\hline 13S02W32DBD1 & 333126 & 911917 & NRCS & -- & 135 & 43 & 92 & $4 / 07 / 2006$ \\
\hline 13S03W10DAA1 & 333506 & 912302 & USGS & 86 & 140 & 47.12 & 93 & $3 / 17 / 2006$ \\
\hline 13S03W11CAB1 & 333503 & 912241 & NRCS & -- & 142 & 51 & 91 & $4 / 07 / 2006$ \\
\hline \multicolumn{9}{|c|}{ Drew County } \\
\hline 11S04W08DBA1 & 334532 & 913136 & USGS & 70 & 160 & 25.14 & 135 & $3 / 16 / 2006$ \\
\hline 11S05W08CCC1 & 334546 & 913837 & USGS & 153 & 185 & 36.48 & 149 & $3 / 16 / 2006$ \\
\hline 12S04W03ABB1 & 334134 & 912946 & USGS & -- & 155 & 24.23 & 131 & $3 / 16 / 2006$ \\
\hline 12S04W25DBB1 & 333739 & 912738 & NRCS & 90 & 149 & 34 & 115 & $4 / 13 / 2006$ \\
\hline 13S04W09ACD1 & 333512 & 913034 & NRCS & 90 & 145 & 19 & 126 & $4 / 13 / 2006$ \\
\hline 13S04W28CDD1 & 333206 & 913100 & USGS & 65 & 139 & 17.63 & 121 & $3 / 16 / 2006$ \\
\hline 13S04W33BAA1 & 333206 & 913100 & USGS & 130 & 138 & 18.14 & 120 & $3 / 16 / 2006$ \\
\hline 13S05W29ADA1 & 333248 & 913747 & USGS & -- & 185 & 40.50 & 145 & $3 / 16 / 2006$ \\
\hline 13S06W03DDC1 & 333545 & 914202 & USGS & 110 & 191 & 62.17 & 129 & $3 / 16 / 2006$ \\
\hline 13S06W21DAA1 & 333324 & 914258 & NRCS & 142 & 207 & 74 & 133 & $4 / 13 / 2006$ \\
\hline 14S04W03ADD1 & 333050 & 912929 & NRCS & 92 & 141 & 27 & 114 & $4 / 13 / 2006$ \\
\hline 14S04W05CBA1 & 333047 & 913218 & NRCS & 90 & 131 & 14 & 117 & $4 / 13 / 2006$ \\
\hline 14S04W05CBC1 & 333042 & 913226 & NRCS & 90 & 131 & 15 & 116 & $4 / 13 / 2006$ \\
\hline 14S04W22CAA1 & 332805 & 912957 & NRCS & 100 & 135 & 13 & 122 & $4 / 13 / 2006$ \\
\hline 14S05W23DCB1 & 332802 & 913512 & USGS & 42 & 161 & 29.77 & 131 & $3 / 16 / 2006$ \\
\hline 15S04W13DAD1 & 332338 & 912730 & NRCS & -- & 131 & 38 & 93 & $4 / 06 / 2006$ \\
\hline \multicolumn{9}{|c|}{ Greene County } \\
\hline 16N03E03BA1 & 360316 & 904516 & USGS & 100 & 260 & 30.73 & 229 & $4 / 18 / 2006$ \\
\hline 16N03E05BBB1 & 360316 & 904750 & NRCS & 105 & 257 & 30.5 & 227 & $4 / 11 / 2006$ \\
\hline 16N03E16DDD1 & 360049 & 904547 & NRCS & 100 & 258 & 26.8 & 231 & $4 / 11 / 2006$ \\
\hline 16N03E29ACC1 & 355926 & 904722 & NRCS & 100 & 257 & 31.5 & 226 & $4 / 11 / 2006$ \\
\hline 16N06E09ABB1 & 360215 & 902651 & NRCS & 90 & 261 & 49.9 & 211 & $4 / 11 / 2006$ \\
\hline 16N06E21BAA1 & 360031 & 902705 & NRCS & 130 & 249 & 27.1 & 222 & $4 / 11 / 2006$ \\
\hline 16N06E28ABB1 & 355938 & 902657 & USGS & -- & 251 & 25.76 & 225 & $4 / 18 / 2006$ \\
\hline 17N03E02BDB1 & 360832 & 904413 & USGS & 115 & 266 & 32.60 & 233 & $4 / 18 / 2006$ \\
\hline 17N04E07AD1 & 360718 & 904122 & NRCS & 100 & 273 & 42.6 & 230 & $4 / 11 / 2006$ \\
\hline 17N04E30CDC1 & 360409 & 904218 & USGS & 100 & 265 & 37.39 & 228 & $4 / 18 / 2006$ \\
\hline 17N06E15ABC1 & 360631 & 902546 & NRCS & 168 & 268 & 31.1 & 237 & $4 / 11 / 2006$ \\
\hline
\end{tabular}


Appendix 1. Information pertaining to water levels measured in wells completed in the Mississippi River Valley alluvial aquifer in eastern Arkansas, spring 2006.-Continued

[USGS, U.S. Geological Survey; NRCS, Natural Resources Conservation Service; --, no data; NGVD of 1929, National Geodetic Vertical Datum of 1929; Horizontal coordinate information is referenced to the North American Datum of 1983 (NAD 83); *, control wells for duplicate measurements by USGS and NRCS]

\begin{tabular}{|c|c|c|c|c|c|c|c|c|}
\hline Station name & $\begin{array}{c}\text { Latitude } \\
\text { (degrees, } \\
\text { minutes, } \\
\text { seconds) }\end{array}$ & $\begin{array}{c}\text { Longitude } \\
\text { (degrees, } \\
\text { minutes, } \\
\text { seconds) }\end{array}$ & $\begin{array}{c}\text { Source } \\
\text { of } \\
\text { data }\end{array}$ & $\begin{array}{c}\text { Depth } \\
\text { of well } \\
\text { (feet) }\end{array}$ & $\begin{array}{l}\text { Land-surface } \\
\text { datum altitude } \\
\text { (feet above } \\
\text { NGVD of } \\
\text { 1929) }\end{array}$ & $\begin{array}{c}\text { Depth to } \\
\text { water } \\
\text { (feet below } \\
\text { land-surface } \\
\text { datum) }\end{array}$ & $\begin{array}{l}\text { Water-level } \\
\text { altitude } \\
\text { (feet above } \\
\text { NGVD of } \\
\text { 1929) }\end{array}$ & $\begin{array}{c}\text { Date of } \\
\text { measure- } \\
\text { ment }\end{array}$ \\
\hline \multicolumn{9}{|c|}{ Greene County-Continued } \\
\hline 17N07E03CCC1 & 360744 & 901951 & NRCS & 87 & 246 & 5.8 & 240 & $4 / 11 / 2006$ \\
\hline 17N07E18ABB1 & 360638 & 902235 & USGS & -- & 245 & 8.86 & 236 & $4 / 18 / 2006$ \\
\hline 17N07E29CBC1 & 360419 & 902201 & NRCS & 80 & 245 & 6.3 & 239 & $4 / 11 / 2006$ \\
\hline 18N04E04AAC1 & 361356 & 903854 & NRCS & 127 & 273 & 32.6 & 240 & $4 / 11 / 2006$ \\
\hline 18N04E21CBD1 & 361052 & 903725 & USGS & -- & 294 & 55.63 & 238 & $4 / 18 / 2006$ \\
\hline 18N06E23ABB1 & 361109 & 902402 & NRCS & 145 & 280 & 14.9 & 265 & $4 / 11 / 2006$ \\
\hline 18N07E17BAB1 & 361203 & 902105 & NRCS & 100 & 262 & 11.3 & 251 & $4 / 11 / 2006$ \\
\hline 18N07E20BBA1 & 361110 & 902113 & USGS & -- & 257 & 10.92 & 246 & $4 / 18 / 2006$ \\
\hline 19N03E26AD1 & 361601 & 904258 & USGS & 100 & 281 & 29.50 & 252 & $4 / 18 / 2006$ \\
\hline 19N03E33DDD1 & 361418 & 904516 & NRCS & 100 & 276 & 36.0 & 240 & $4 / 11 / 2006$ \\
\hline 19N05E34AAD1 & 361437 & 903102 & NRCS & 130 & 282 & 34.2 & 248 & $4 / 11 / 2006$ \\
\hline \multicolumn{9}{|c|}{ Independence County } \\
\hline 11N04W02ABB1 & 353650 & 912416 & NRCS & -- & 227 & 10.2 & 217 & $4 / 04 / 2006$ \\
\hline 12N04W14DD1 & 353929 & 912236 & USGS & 60 & 231 & 27.03 & 204 & $3 / 23 / 2006$ \\
\hline 12N04W34CBB1 & 353720 & 912513 & USGS & -- & 231 & 23.51 & 207 & $3 / 23 / 2006$ \\
\hline 12N05W36AAA1 & 353738 & 912827 & USGS & -- & 236 & 25.64 & 210 & $3 / 23 / 2006$ \\
\hline 14N03W12CAB1 & 355152 & 911541 & NRCS & -- & 230 & 2.8 & 227 & $4 / 04 / 2006$ \\
\hline 14N03W14CBB1 & 355101 & 911703 & NRCS & -- & 235 & 2.4 & 233 & $4 / 04 / 2006$ \\
\hline 14N03W14DAA2 & 355107 & 911602 & USGS & -- & 230 & 4.89 & 225 & $3 / 23 / 2006$ \\
\hline 14N03W14DBB1 & 355106 & 911640 & USGS & 65 & 230 & 5.93 & 224 & $3 / 23 / 2006$ \\
\hline \multicolumn{9}{|c|}{ Jackson County } \\
\hline 09N01W15DDD1 & 352357 & 910433 & NRCS & 90 & 220 & 54.6 & 165 & $4 / 20 / 2006$ \\
\hline 09N01W22ADD1 & 352332 & 910433 & USGS & 125 & 215 & 61.72 & 153 & $3 / 27 / 2006$ \\
\hline 09N01W30BAC1 & 352258 & 910813 & NRCS & 120 & 218 & 44.5 & 174 & $4 / 20 / 2006$ \\
\hline 09N02W32BBB1 & 352215 & 911344 & NRCS & 100 & 220 & 34.0 & 186 & $4 / 20 / 2006$ \\
\hline 09N02W32CBB1 & 352152 & 911348 & USGS & 117 & 220 & 30.29 & 190 & $3 / 27 / 2006$ \\
\hline 10N01W05ADD1 & 353132 & 910702 & NRCS & -- & 227 & 47.3 & 180 & $4 / 20 / 2006$ \\
\hline 10N01W10ABA1 & 353055 & 910445 & NRCS & 135 & 223 & 59.2 & 164 & $4 / 20 / 2006$ \\
\hline 10N02W29ABB1 & 352829 & 911312 & USGS & -- & 227 & 27.94 & 199 & $3 / 27 / 2006$ \\
\hline 11N01W26AAD1* & 353330 & 910323 & USGS & 95 & 227 & 67.06 & 160 & $3 / 24 / 2006$ \\
\hline 11N01W26AAD1* & 353330 & 910323 & NRCS & 95 & 227 & 66.2 & 161 & $4 / 20 / 2006$ \\
\hline 11N01W29AAD1 & 353339 & 910635 & USGS & 97 & 225 & 39.07 & 186 & $3 / 24 / 2006$ \\
\hline 11N02W25BBD1 & 353322 & 910855 & NRCS & 100 & 221 & 26.0 & 195 & $4 / 20 / 2006$ \\
\hline 11N03W05CAB1 & 353655 & 912008 & NRCS & 95 & 225 & 21.1 & 204 & $4 / 12 / 2006$ \\
\hline
\end{tabular}


Appendix 1. Information pertaining to water levels measured in wells completed in the Mississippi River Valley alluvial aquifer in eastern Arkansas, spring 2006.-Continued

[USGS, U.S. Geological Survey; NRCS, Natural Resources Conservation Service; --, no data; NGVD of 1929, National Geodetic Vertical Datum of 1929; Horizontal coordinate information is referenced to the North American Datum of 1983 (NAD 83); *, control wells for duplicate measurements by USGS and NRCS]

\begin{tabular}{|c|c|c|c|c|c|c|c|c|}
\hline Station name & $\begin{array}{c}\text { Latitude } \\
\text { (degrees, } \\
\text { minutes, } \\
\text { seconds) }\end{array}$ & $\begin{array}{c}\text { Longitude } \\
\text { (degrees, } \\
\text { minutes, } \\
\text { seconds) }\end{array}$ & $\begin{array}{c}\text { Source } \\
\text { of } \\
\text { data }\end{array}$ & $\begin{array}{c}\text { Depth } \\
\text { of well } \\
\text { (feet) }\end{array}$ & $\begin{array}{l}\text { Land-surface } \\
\text { datum altitude } \\
\text { (feet above } \\
\text { NGVD of } \\
\text { 1929) }\end{array}$ & $\begin{array}{c}\text { Depth to } \\
\text { water } \\
\text { (feet below } \\
\text { land-surface } \\
\text { datum) }\end{array}$ & $\begin{array}{l}\text { Water-level } \\
\text { altitude } \\
\text { (feet above } \\
\text { NGVD of } \\
\text { 1929) }\end{array}$ & $\begin{array}{c}\text { Date of } \\
\text { measure- } \\
\text { ment }\end{array}$ \\
\hline \multicolumn{9}{|c|}{ Jackson County-Continued } \\
\hline 11N03W06DAB1 & 353655 & 912009 & USGS & 100 & 223 & 22.23 & 201 & $3 / 24 / 2006$ \\
\hline 12N01W11BCB1 & 354127 & 910416 & NRCS & 110 & 233 & 38.2 & 195 & $4 / 12 / 2006$ \\
\hline 12N01W30CCC2 & 353812 & 910821 & NRCS & 140 & 227 & 33.0 & 194 & $4 / 12 / 2006$ \\
\hline 12N01W36CBC1 & 353724 & 910317 & NRCS & 120 & 236 & 52.9 & 183 & $4 / 12 / 2006$ \\
\hline $12 \mathrm{~N} 02 \mathrm{~W} 25 \mathrm{ABB} 2$ & 353910 & 910852 & USGS & -- & 234 & 33.24 & 201 & $3 / 24 / 2006$ \\
\hline 13N01W20AAA1 & 354514 & 910627 & USGS & 147 & 242 & 39.26 & 203 & $3 / 27 / 2006$ \\
\hline 13N03W15CDD1 & 354526 & 911749 & USGS & -- & 232 & 16.93 & 215 & $3 / 27 / 2006$ \\
\hline 13N03W15DCB1 & 354540 & 911718 & NRCS & 80 & 238 & 17.9 & 220 & $4 / 12 / 2006$ \\
\hline 13N03W36ABB1 & 354337 & 911532 & NRCS & 110 & 241 & 16.6 & 224 & $4 / 12 / 2006$ \\
\hline 14N01W08AAA1 & 355216 & 910623 & NRCS & 80 & 252 & 36.0 & 216 & $4 / 12 / 2006$ \\
\hline 14N01W09AAA1 & 355220 & 910515 & USGS & -- & 251 & 42.43 & 209 & $3 / 27 / 2006$ \\
\hline 14N01W19BBB1 & 355032 & 910823 & NRCS & 100 & 246 & 32.0 & 214 & $4 / 12 / 2006$ \\
\hline 14N01W26BCB1 & 354922 & 910407 & NRCS & 110 & 247 & 43.3 & 204 & $4 / 12 / 2006$ \\
\hline 14N01W33CCD1 & 354759 & 910610 & NRCS & 100 & 245 & 39.3 & 206 & $4 / 12 / 2006$ \\
\hline 14N02W22BBC1 & 355026 & 911145 & NRCS & 100 & 250 & 28.0 & 222 & $4 / 12 / 2006$ \\
\hline \multicolumn{9}{|c|}{ Jefferson County } \\
\hline 03S08W24BBC1 & 342620 & 914953 & USGS & 135 & 202 & 51.03 & 151 & $4 / 03 / 2006$ \\
\hline 03S09W06DDA1 & 342840 & 920037 & USGS & -- & 225 & 37.94 & 187 & $4 / 03 / 2006$ \\
\hline 03S09W14BCD1 & 342712 & 915713 & NRCS & -- & 220 & 56.0 & 164 & $4 / 06 / 2006$ \\
\hline 03S09W22AAA1 & 342640 & 915728 & NRCS & 100 & 218 & 40.7 & 177 & $4 / 06 / 2006$ \\
\hline 03S09W29CBD1 & 342517 & 920023 & USGS & -- & 216 & 29.11 & 187 & 4/03/2006 \\
\hline 03S09W36ACC1 & 342428 & 915555 & NRCS & -- & 214 & 27.9 & 186 & $4 / 07 / 2006$ \\
\hline 03S10W25BCA2 & 342537 & 920242 & NRCS & -- & 216 & 18.8 & 197 & $4 / 06 / 2006$ \\
\hline 03S10W26BBB2 & 342427 & 920250 & NRCS & -- & 215 & 15.7 & 199 & $4 / 06 / 2006$ \\
\hline 04S07W35DDB1 & 341836 & 914347 & NRCS & -- & 185 & 27.3 & 158 & $4 / 06 / 2006$ \\
\hline 04S08W13DCB1 & 342123 & 914926 & USGS & 110 & 204 & 48.34 & 156 & 4/03/2006 \\
\hline 04S09W02CBD1 & 342325 & 915717 & NRCS & 110 & 212 & 33.3 & 179 & $4 / 06 / 2006$ \\
\hline 04S09W32DDA1 & 341859 & 920009 & NRCS & -- & 212 & 23.0 & 189 & $4 / 06 / 2006$ \\
\hline 05S06W31CAA1 & 341330 & 914206 & USGS & -- & 189.22 & 19.85 & 169 & $4 / 04 / 2006$ \\
\hline 05S07W29DDD1 & 341411 & 914654 & NRCS & 110 & 194 & 14.2 & 180 & $4 / 06 / 2006$ \\
\hline 05S08W12DAA1 & 341712 & 914907 & USGS & 101 & 194.25 & 18.98 & 175 & $4 / 03 / 2006$ \\
\hline 06S05W15BCA1 & 341023 & 913245 & USGS & 120 & 177.14 & 18.58 & 159 & $4 / 04 / 2006$ \\
\hline 06S06W23AAD1 & 341007 & 913712 & USGS & 107 & 189.01 & 21.23 & 168 & $4 / 04 / 2006$ \\
\hline 06S07W14BAA1 & 341125 & 914426 & USGS & 110 & 199 & 16.07 & 183 & $4 / 04 / 2006$ \\
\hline
\end{tabular}


Appendix 1. Information pertaining to water levels measured in wells completed in the Mississippi River Valley alluvial aquifer in eastern Arkansas, spring 2006.-Continued

[USGS, U.S. Geological Survey; NRCS, Natural Resources Conservation Service; --, no data; NGVD of 1929, National Geodetic Vertical Datum of 1929; Horizontal coordinate information is referenced to the North American Datum of 1983 (NAD 83); *, control wells for duplicate measurements by USGS and NRCS]

\begin{tabular}{|c|c|c|c|c|c|c|c|c|}
\hline Station name & $\begin{array}{c}\text { Latitude } \\
\text { (degrees, } \\
\text { minutes, } \\
\text { seconds) }\end{array}$ & $\begin{array}{c}\text { Longitude } \\
\text { (degrees, } \\
\text { minutes, } \\
\text { seconds) }\end{array}$ & $\begin{array}{c}\text { Source } \\
\text { of } \\
\text { data }\end{array}$ & $\begin{array}{c}\text { Depth } \\
\text { of well } \\
\text { (feet) }\end{array}$ & $\begin{array}{l}\text { Land-surface } \\
\text { datum altitude } \\
\text { (feet above } \\
\text { NGVD of } \\
\text { 1929) }\end{array}$ & $\begin{array}{c}\text { Depth to } \\
\text { water } \\
\text { (feet below } \\
\text { land-surface } \\
\text { datum) }\end{array}$ & $\begin{array}{l}\text { Water-level } \\
\text { altitude } \\
\text { (feet above } \\
\text { NGVD of } \\
\text { 1929) }\end{array}$ & $\begin{array}{c}\text { Date of } \\
\text { measure- } \\
\text { ment }\end{array}$ \\
\hline \multicolumn{9}{|c|}{ Jefferson County-Continued } \\
\hline 07S07W16BAA1 & 340722 & 914828 & NRCS & -- & 190 & 28.0 & 162 & $4 / 06 / 2006$ \\
\hline 07S08W06BAA1 & 340859 & 915647 & USGS & 160 & 202.31 & 18.65 & 184 & $4 / 04 / 2006$ \\
\hline \multicolumn{9}{|c|}{ Lawrence County } \\
\hline 15N01E11ADD1 & 355657 & 905638 & NRCS & 100 & 255 & 44.4 & 211 & $4 / 18 / 2006$ \\
\hline 15N01E26DDA1 & 355402 & 905639 & USGS & 100 & 251 & 51.93 & 199 & $4 / 17 / 2006$ \\
\hline 15N01W03BAB1 & 355831 & 910441 & NRCS & 105 & 259 & 36.2 & 223 & $4 / 18 / 2006$ \\
\hline 15N01W35CBB1 & 355336 & 910356 & USGS & -- & 250 & 44.98 & 205 & $4 / 17 / 2006$ \\
\hline 16N01E11DAC2 & 360203 & 905639 & USGS & -- & 262 & 46.76 & 215 & $4 / 17 / 2006$ \\
\hline 16N01E35AAA1 & 355908 & 905632 & NRCS & 105 & 256 & 49.2 & 207 & $4 / 18 / 2006$ \\
\hline 16N01W30DDC1 & 355937 & 910723 & NRCS & 105 & 255 & 21.6 & 233 & $4 / 18 / 2006$ \\
\hline 16N02E09AAD1 & 360219 & 905212 & NRCS & 110 & 261 & 40.3 & 221 & $4 / 18 / 2006$ \\
\hline 16N02E34CBB1 & 355831 & 905208 & NRCS & 100 & 255 & 48.1 & 207 & $4 / 18 / 2006$ \\
\hline 17N01E02BBA1 & 360901 & 905707 & NRCS & 90 & 260 & 15.0 & 245 & $4 / 18 / 2006$ \\
\hline 17N01W36AAB1 & 360435 & 910158 & NRCS & 85 & 257 & 13.1 & 244 & $4 / 18 / 2006$ \\
\hline 17N02E04DCA1 & 360758 & 905224 & NRCS & 110 & 270 & 40.9 & 229 & $4 / 18 / 2006$ \\
\hline 17N02E19CDC1 & 360516 & 905449 & USGS & 105 & 265 & 38.94 & 226 & $4 / 17 / 2006$ \\
\hline 17N02E25CBD1 & 360423 & 904948 & NRCS & 100 & 265 & 38.1 & 227 & $4 / 18 / 2006$ \\
\hline \multicolumn{9}{|c|}{ Lee County } \\
\hline 01N01E04AAB1 & 344358 & 910015 & NRCS & 140 & 175 & 29.3 & 146 & $5 / 02 / 2006$ \\
\hline 01N01E09CCC1 & 344215 & 910054 & NRCS & 140 & 182 & 32.5 & 150 & $5 / 02 / 2006$ \\
\hline 01N01E24CBD1 & 344033 & 905729 & NRCS & 140 & 185 & 16.3 & 169 & $5 / 03 / 2006$ \\
\hline 01N02E01ADD1 & 344330 & 905016 & NRCS & 140 & 207 & 28.0 & 179 & $5 / 02 / 2006$ \\
\hline 01N02E11BAB1 & 344255 & 905208 & NRCS & 140 & 202 & 32.0 & 170 & $4 / 22 / 2006$ \\
\hline 01N02E12ABB1 & 344254 & 905040 & NRCS & 140 & 206 & 27.0 & 179 & $5 / 02 / 2006$ \\
\hline 01N02E22CBA1 & 344056 & 905318 & NRCS & 140 & 200 & 28.5 & 172 & $5 / 02 / 2006$ \\
\hline 01N02E33CBB1 & 343858 & 905434 & NRCS & 140 & 186 & 16.0 & 170 & $5 / 02 / 2006$ \\
\hline 01N02E33CCB1 & 343851 & 905433 & NRCS & 140 & 185 & 14.0 & 171 & $5 / 02 / 2006$ \\
\hline 01N03E02BBC1 & 344339 & 904601 & USGS & 168 & 236.43 & 48.57 & 188 & $3 / 21 / 2006$ \\
\hline 01N03E27ADD1 & 343952 & 904605 & NRCS & 120 & 204 & 16.0 & 188 & $5 / 02 / 2006$ \\
\hline 01N03E35BBA1 & 343923 & 904549 & USGS & 120 & 202 & 10.24 & 192 & $3 / 21 / 2006$ \\
\hline 02N01E21BAA1 & 344633 & 910005 & NRCS & 140 & 185 & 35.3 & 150 & $5 / 02 / 2006$ \\
\hline 02N01E23BAA2 & 344632 & 905820 & USGS & 137 & 202 & 50.18 & 152 & $3 / 21 / 2006$ \\
\hline 02N01W12BAA1 & 344828 & 910330 & USGS & 95 & 185 & 43.52 & 141 & $3 / 21 / 2006$ \\
\hline 02N01W34DDC1 & 344410 & 910520 & NRCS & 140 & 180 & 52.0 & 128 & $5 / 02 / 2006$ \\
\hline
\end{tabular}


Appendix 1. Information pertaining to water levels measured in wells completed in the Mississippi River Valley alluvial aquifer in eastern Arkansas, spring 2006.-Continued

[USGS, U.S. Geological Survey; NRCS, Natural Resources Conservation Service; --, no data; NGVD of 1929, National Geodetic Vertical Datum of 1929; Horizontal coordinate information is referenced to the North American Datum of 1983 (NAD 83); *, control wells for duplicate measurements by USGS and NRCS]

\begin{tabular}{|c|c|c|c|c|c|c|c|c|}
\hline Station name & $\begin{array}{l}\text { Latitude } \\
\text { (degrees, } \\
\text { minutes, } \\
\text { seconds) }\end{array}$ & $\begin{array}{c}\text { Longitude } \\
\text { (degrees, } \\
\text { minutes, } \\
\text { seconds) }\end{array}$ & $\begin{array}{c}\text { Source } \\
\text { of } \\
\text { data }\end{array}$ & $\begin{array}{c}\text { Depth } \\
\text { of well } \\
\text { (feet) }\end{array}$ & $\begin{array}{l}\text { Land-surface } \\
\text { datum altitude } \\
\text { (feet above } \\
\text { NGVD of } \\
\text { 1929) }\end{array}$ & $\begin{array}{c}\text { Depth to } \\
\text { water } \\
\text { (feet below } \\
\text { land-surface } \\
\text { datum) }\end{array}$ & $\begin{array}{l}\text { Water-level } \\
\text { altitude } \\
\text { (feet above } \\
\text { NGVD of } \\
\text { 1929) }\end{array}$ & $\begin{array}{c}\text { Date of } \\
\text { measure- } \\
\text { ment }\end{array}$ \\
\hline \multicolumn{9}{|c|}{ Lee County-Continued } \\
\hline 02N02E08ADC1 & 344807 & 905339 & USGS & 120 & 201 & 43.46 & 158 & $3 / 21 / 2006$ \\
\hline 02N02E21ABC1 & 344622 & 905358 & USGS & 120 & 200 & 37.73 & 162 & $3 / 21 / 2006$ \\
\hline 02N02E22BBB1 & 344628 & 905327 & NRCS & 140 & 200 & 28.0 & 172 & $5 / 02 / 2006$ \\
\hline 02N02E36DDC1 & 344355 & 905020 & NRCS & 140 & 205 & 26.5 & 179 & $5 / 02 / 2006$ \\
\hline 02N03E08AAD1 & 344811 & 904838 & USGS & 100 & 211 & 43.89 & 167 & $3 / 21 / 2006$ \\
\hline 02N03E09DDD1 & 344723 & 904707 & NRCS & 120 & 220 & 51.0 & 169 & $5 / 02 / 2006$ \\
\hline 02N03E29CAD1 & 344500 & 904846 & NRCS & 140 & 220 & 50.0 & 170 & $5 / 02 / 2006$ \\
\hline 02N04E03ABD1 & 344855 & 903954 & NRCS & 140 & 192 & 24.5 & 168 & $5 / 03 / 2006$ \\
\hline 02N04E15DAC1 & 344637 & 903950 & USGS & 60 & 192 & 17.62 & 174 & $3 / 21 / 2006$ \\
\hline 03N01E16CBA1 & 345222 & 910040 & USGS & 110 & 202 & 63.57 & 138 & $3 / 21 / 2006$ \\
\hline 03N01E32BCC1 & 344951 & 910150 & NRCS & 140 & 200 & 62.0 & 138 & $5 / 02 / 2006$ \\
\hline 03N02E12CDC1 & 345239 & 905053 & NRCS & 140 & 210 & 41.0 & 169 & $5 / 02 / 2006$ \\
\hline 03N02E13BBA1 & 345237 & 905107 & USGS & 65 & 212 & 49.98 & 162 & $3 / 22 / 2006$ \\
\hline 03N02E21CBC1 & 345111 & 905428 & NRCS & 140 & 209 & 54.0 & 155 & $5 / 02 / 2006$ \\
\hline 03N02E29DAD1 & 345014 & 905430 & USGS & 135 & 205 & 42.71 & 162 & $3 / 21 / 2006$ \\
\hline 03N03E05CDD1 & 345327 & 904837 & NRCS & 110 & 204 & 49.5 & 155 & $5 / 01 / 2006$ \\
\hline 03N03E18DAB1 & 345206 & 904919 & NRCS & 140 & 196 & 29.0 & 167 & $5 / 02 / 2006$ \\
\hline 03N03E32CAB1 & 344933 & 904926 & USGS & 116 & 204 & 49.92 & 154 & $3 / 22 / 2006$ \\
\hline 03N04E07CBB1 & 345245 & 904312 & NRCS & 140 & 200 & 30.0 & 170 & $5 / 01 / 2006$ \\
\hline 03N05E14DDA1 & 345148 & 903203 & USGS & 120 & 193 & 13.56 & 179 & $3 / 21 / 2006$ \\
\hline 03N05E26ADC1 & 345020 & 903215 & NRCS & 140 & 185 & 7.0 & 178 & $5 / 03 / 2006$ \\
\hline \multicolumn{9}{|c|}{ Lincoln County } \\
\hline 07S06W03CCA2 & 340828 & 914114 & NRCS & 110 & 190 & 19 & 171 & $4 / 12 / 2006$ \\
\hline 07S07W36CBD1 & 340411 & 914529 & NRCS & 123 & 183 & 38 & 145 & $4 / 12 / 2006$ \\
\hline 08S04W06ABD1 & 340341 & 913116 & NRCS & 95 & 171 & 20 & 151 & $4 / 12 / 2006$ \\
\hline 08S04W08BBB2 & 340254 & 913101 & USGS & 65.2 & 171 & 21.87 & 149 & $3 / 20 / 2006$ \\
\hline 08S04W29ABC1 & 340021 & 913044 & NRCS & 100 & 176 & 41 & 135 & $4 / 12 / 2006$ \\
\hline 08S04W31CBA1 & 335901 & 913150 & USGS & 99 & 161.9 & 32.63 & 129 & $3 / 20 / 2006$ \\
\hline 08S05W12AAD1 & 340246 & 913214 & NRCS & 83 & 165 & 22 & 143 & $4 / 12 / 2006$ \\
\hline 08S05W21DCD1 & 340027 & 913533 & NRCS & 120 & 169 & 35 & 134 & $4 / 12 / 2006$ \\
\hline 08S05W32DCC1 & 335840 & 913644 & NRCS & 100 & 172 & 43 & 129 & $4 / 12 / 2006$ \\
\hline 08S06W02ACB1 & 340339 & 913958 & USGS & 68 & 181.03 & 42.25 & 139 & $3 / 20 / 2006$ \\
\hline 08S07W05DDD1 & 340301 & 914903 & USGS & 97 & 190 & 29.96 & 160 & $3 / 20 / 2006$ \\
\hline 09S04W06CBB1 & 335721 & 913252 & NRCS & 110 & 163 & 40 & 123 & $4 / 12 / 2006$ \\
\hline
\end{tabular}


Appendix 1. Information pertaining to water levels measured in wells completed in the Mississippi River Valley alluvial aquifer in eastern Arkansas, spring 2006.-Continued

[USGS, U.S. Geological Survey; NRCS, Natural Resources Conservation Service; --, no data; NGVD of 1929, National Geodetic Vertical Datum of 1929; Horizontal coordinate information is referenced to the North American Datum of 1983 (NAD 83); *, control wells for duplicate measurements by USGS and NRCS]

\begin{tabular}{|c|c|c|c|c|c|c|c|c|}
\hline Station name & $\begin{array}{c}\text { Latitude } \\
\text { (degrees, } \\
\text { minutes, } \\
\text { seconds) }\end{array}$ & $\begin{array}{c}\text { Longitude } \\
\text { (degrees, } \\
\text { minutes, } \\
\text { seconds) }\end{array}$ & $\begin{array}{c}\text { Source } \\
\text { of } \\
\text { data }\end{array}$ & $\begin{array}{c}\text { Depth } \\
\text { of well } \\
\text { (feet) }\end{array}$ & $\begin{array}{l}\text { Land-surface } \\
\text { datum altitude } \\
\text { (feet above } \\
\text { NGVD of } \\
\text { 1929) }\end{array}$ & $\begin{array}{c}\text { Depth to } \\
\text { water } \\
\text { (feet below } \\
\text { land-surface } \\
\text { datum) }\end{array}$ & $\begin{array}{l}\text { Water-level } \\
\text { altitude } \\
\text { (feet above } \\
\text { NGVD of } \\
\text { 1929) }\end{array}$ & $\begin{array}{c}\text { Date of } \\
\text { measure- } \\
\text { ment }\end{array}$ \\
\hline \multicolumn{9}{|c|}{ Lincoln County-Continued } \\
\hline 09S05W14ABC1 & 335553 & 913439 & USGS & 98 & 172.5 & 37.21 & 135 & $3 / 20 / 2006$ \\
\hline 09S05W17BCB1 & 335552 & 913820 & USGS & 97 & 171 & 41.09 & 130 & $3 / 20 / 2006$ \\
\hline 09S05W19CCC1 & 335428 & 913941 & NRCS & 110 & 171 & 31 & 140 & $4 / 12 / 2006$ \\
\hline 09S06W04BCD1 & 335821 & 914346 & USGS & 62.6 & 181 & 38.12 & 143 & $3 / 20 / 2006$ \\
\hline 09S06W04BDD1 & 335759 & 914335 & NRCS & 100 & 178 & 36 & 142 & $4 / 12 / 2006$ \\
\hline 09S06W23CDB1 & 335440 & 914136 & USGS & 70 & 175 & 29.73 & 145 & $3 / 20 / 2006$ \\
\hline 10S05W06DCC1 & 335155 & 913908 & USGS & 65 & 175 & 29.92 & 145 & $3 / 20 / 2006$ \\
\hline \multicolumn{9}{|c|}{ Lonoke County } \\
\hline 01N08W03DDA1 & 344411 & 915050 & NRCS & -- & 229 & 139.2 & 90 & $4 / 17 / 2006$ \\
\hline 01N09W07DAA1 & 344337 & 920030 & NRCS & -- & 240 & 47.0 & 193 & $4 / 17 / 2006$ \\
\hline 01N09W13DAB1 & 344235 & 915517 & USGS & 150 & 226 & 86.74 & 139 & $4 / 13 / 2006$ \\
\hline 01N10W15CDA1 & 344236 & 920415 & NRCS & 100 & 240 & 24.7 & 215 & $4 / 17 / 2006$ \\
\hline 01S06W31ABB1 & 343459 & 914131 & USGS & 120 & 200 & 78.73 & 121 & $4 / 13 / 2006$ \\
\hline 01S06W32BBB1 & 343501 & 914056 & NRCS & -- & 201 & 77.0 & 124 & $4 / 17 / 2006$ \\
\hline 01S07W12ABA1 & 343834 & 914230 & USGS & 140 & 207 & 69.93 & 137 & $4 / 13 / 2006$ \\
\hline 01S08W24CDD1 & 343606 & 914912 & USGS & 127 & 210 & 81.0 & 129 & $4 / 13 / 2006$ \\
\hline 01S09W02DDD1 & 343857 & 915624 & NRCS & -- & 230 & 83.0 & 147 & $4 / 17 / 2006$ \\
\hline 01S09W36CCC $1 *$ & 343435 & 915619 & USGS & 95 & 220 & 62.51 & 157 & $4 / 13 / 2006$ \\
\hline 01S09W36CCC $1 *$ & 343435 & 915619 & NRCS & 95 & 220 & 62.5 & 158 & $4 / 17 / 2006$ \\
\hline 01S10W01ACB1 & 343927 & 920215 & USGS & -- & 236 & 45.92 & 190 & $4 / 13 / 2006$ \\
\hline 02N07W07DAA1 & 344845 & 914707 & NRCS & -- & 232 & 140.2 & 92 & $4 / 17 / 2006$ \\
\hline 02N07W16BAB1 & 344815 & 914540 & USGS & 184 & 240 & 137.64 & 102 & $4 / 13 / 2006$ \\
\hline 02N08W16ABC1 & 344806 & 915114 & USGS & 170 & 230 & 128.39 & 102 & $4 / 13 / 2006$ \\
\hline 02N08W23CAB1 & 344659 & 915118 & NRCS & -- & 229 & 129.7 & 99 & $4 / 17 / 2006$ \\
\hline 02N09W02BDB1 & 344955 & 915841 & USGS & 140 & 251 & 126.04 & 125 & $4 / 13 / 2006$ \\
\hline 02N10W15ACC1 & 344807 & 920353 & NRCS & 135 & 241 & 31.5 & 210 & $4 / 17 / 2006$ \\
\hline 02S07W05CDC1 & 343326 & 914715 & NRCS & -- & 205 & 70.4 & 135 & $4 / 17 / 2006$ \\
\hline 02S07W10CCB1 & 343246 & 914525 & USGS & -- & 201 & 62.39 & 139 & $4 / 13 / 2006$ \\
\hline 02S07W20ACD1 & 343112 & 914655 & NRCS & -- & 201 & 60.6 & 140 & $4 / 17 / 2006$ \\
\hline 02S08W13BBB1 & 343232 & 914935 & USGS & -- & 200 & 59.85 & 140 & $4 / 13 / 2006$ \\
\hline 02S08W34DBB1 & 343003 & 915150 & USGS & -- & 214 & 66.62 & 147 & $4 / 13 / 2006$ \\
\hline 02S09W26DC1 & 343019 & 915643 & NRCS & 100 & 216 & 50.0 & 166 & $4 / 17 / 2006$ \\
\hline 02S09W30CDD1 & 343014 & 920116 & USGS & 80 & 226 & 39.44 & 187 & $4 / 13 / 2006$ \\
\hline 03N07W08BDB1 & 345407 & 914638 & USGS & 125 & 250 & 96.48 & 154 & $2 / 09 / 2006$ \\
\hline
\end{tabular}


Appendix 1. Information pertaining to water levels measured in wells completed in the Mississippi River Valley alluvial aquifer in eastern Arkansas, spring 2006.-Continued

[USGS, U.S. Geological Survey; NRCS, Natural Resources Conservation Service; --, no data; NGVD of 1929, National Geodetic Vertical Datum of 1929; Horizontal coordinate information is referenced to the North American Datum of 1983 (NAD 83); *, control wells for duplicate measurements by USGS and NRCS]

\begin{tabular}{|c|c|c|c|c|c|c|c|c|}
\hline Station name & $\begin{array}{c}\text { Latitude } \\
\text { (degrees, } \\
\text { minutes, } \\
\text { seconds) }\end{array}$ & $\begin{array}{l}\text { Longitude } \\
\text { (degrees, } \\
\text { minutes, } \\
\text { seconds) }\end{array}$ & $\begin{array}{c}\text { Source } \\
\text { of } \\
\text { data }\end{array}$ & $\begin{array}{l}\text { Depth } \\
\text { of well } \\
\text { (feet) }\end{array}$ & $\begin{array}{c}\text { Land-surface } \\
\text { datum altitude } \\
\text { (feet above } \\
\text { NGVD of } \\
\text { 1929) }\end{array}$ & $\begin{array}{c}\text { Depth to } \\
\text { water } \\
\text { (feet below } \\
\text { land-surface } \\
\text { datum) }\end{array}$ & $\begin{array}{c}\text { Water-level } \\
\text { altitude } \\
\text { (feet above } \\
\text { NGVD of } \\
\text { 1929) }\end{array}$ & $\begin{array}{c}\text { Date of } \\
\text { measure- } \\
\text { ment }\end{array}$ \\
\hline \multicolumn{9}{|c|}{ Lonoke County-Continued } \\
\hline 03N07W15DBC2 & 345253 & 914417 & USGS & 144.5 & 227 & 82.43 & 145 & 4/13/2006 \\
\hline 03N07W29ADA1 & 345129 & 914558 & USGS & 120 & 234 & 94.22 & 140 & 2/09/2006 \\
\hline 03N07W29CDD1 & 345057 & 914632 & NRCS & 157 & 232 & 95.0 & 137 & $4 / 17 / 2006$ \\
\hline 03N07W35CDC2 & 344957 & 914332 & USGS & -- & 232 & 116.1 & 116 & $4 / 13 / 2006$ \\
\hline 03N08W03BAA1 & 345519 & 915054 & USGS & 162 & 260 & 94.87 & 165 & 2/09/2006 \\
\hline 03N08W03CCC1 & 345430 & 915123 & USGS & 162 & 260 & 101.86 & 158 & 2/09/2006 \\
\hline 03N08W05CCC1 & 345429 & 915323 & USGS & 130 & 257 & 81.08 & 176 & 2/09/2006 \\
\hline 03N08W08ABA1 & 345427 & 915248 & USGS & 150 & 258 & 93.11 & 165 & 2/09/2006 \\
\hline 03N08W10ACB1 & 345415 & 915053 & USGS & 150 & 250 & 91.04 & 159 & 2/09/2006 \\
\hline 03N08W10ADD1 & 345401 & 915023 & USGS & 165 & 250 & 89.88 & 160 & 2/10/2006 \\
\hline 03N08W11ABD1 & 345419 & 914936 & USGS & 160 & 260 & 103.86 & 156 & $2 / 10 / 2006$ \\
\hline 03N08W11ACA1 & 345413 & 914934 & USGS & 144 & 256 & 101.53 & 154 & $2 / 10 / 2006$ \\
\hline 03N08W21BCC1 & 345220 & 915220 & USGS & 155 & 247 & 81.66 & 165 & 4/13/2006 \\
\hline 03N08W29BBB1 & 345147 & 915333 & USGS & 152.2 & 249 & 112.33 & 137 & 2/09/2006 \\
\hline 03N08W29BCC1 & 345125 & 915333 & USGS & 150 & 250 & 128.81 & 121 & 2/09/2006 \\
\hline 03N08W32ABB1 & 345057 & 915257 & USGS & 154 & 250 & 118.35 & 132 & $5 / 02 / 2006$ \\
\hline 03N08W32ABB2 & 345057 & 915259 & USGS & 154 & 250 & 118.68 & 131 & 4/13/2006 \\
\hline 03N08W34ADD1 & 345035 & 915028 & USGS & 130 & 240 & 122.50 & 118 & $2 / 09 / 2006$ \\
\hline 04N08W05ACA1 & 350020 & 915247 & USGS & 138 & 238 & 45.64 & 192 & 2/10/2006 \\
\hline 04N08W10BDD1 & 345917 & 915055 & USGS & 130 & 218 & 26.15 & 192 & $2 / 10 / 2006$ \\
\hline 04N08W15BCB2 & 345833 & 915121 & USGS & 104 & 225 & 34.86 & 190 & 4/13/2006 \\
\hline 04N08W16DCC1 & 345757 & 915154 & USGS & 155 & 225 & 46.78 & 178 & $2 / 10 / 2006$ \\
\hline 04N08W26AAD1 & 345652 & 914917 & USGS & 130 & 246 & 72.86 & 173 & 2/09/2006 \\
\hline 04N08W28CAC1 & 345620 & 915216 & USGS & 140.5 & 235 & 55.00 & 180 & 2/09/2006 \\
\hline 04N08W28CAD1 & 345626 & 915204 & USGS & 115 & 249 & 70.26 & 179 & 2/09/2006 \\
\hline 04N08W28CCC1 & 345615 & 915225 & USGS & 137 & 240 & 60.39 & 180 & 2/09/2006 \\
\hline 04N08W36DBB1 & 345541 & 914914 & USGS & 130 & 259 & 93.71 & 165 & 2/09/2006 \\
\hline \multicolumn{9}{|c|}{ Mississippi County } \\
\hline 10N08E21ABA1 & 352852 & 901415 & NRCS & 110 & 224 & 25 & 199 & $4 / 18 / 2006$ \\
\hline 10N08E21BDC1 & 352830 & 901407 & NRCS & 100 & 224 & 25 & 199 & $4 / 18 / 2006$ \\
\hline 10N08E22ABA2 & 352851 & 901312 & USGS & 100 & 224 & 24.04 & 200 & $4 / 20 / 2006$ \\
\hline 10N09E08ACC1 & 352949 & 900926 & USGS & 110 & 230 & 15.84 & 214 & 4/20/2006 \\
\hline 11N09E34BBB1 & 353218 & 900715 & USGS & 94 & 235 & 16.70 & 218 & $4 / 20 / 2006$ \\
\hline 11N10E09BCB1 & 353530 & 900202 & NRCS & 110 & 236 & 20 & 216 & $4 / 14 / 2006$ \\
\hline
\end{tabular}


Appendix 1. Information pertaining to water levels measured in wells completed in the Mississippi River Valley alluvial aquifer in eastern Arkansas, spring 2006.-Continued

[USGS, U.S. Geological Survey; NRCS, Natural Resources Conservation Service; --, no data; NGVD of 1929, National Geodetic Vertical Datum of 1929; Horizontal coordinate information is referenced to the North American Datum of 1983 (NAD 83); *, control wells for duplicate measurements by USGS and NRCS]

\begin{tabular}{|c|c|c|c|c|c|c|c|c|}
\hline Station name & $\begin{array}{c}\text { Latitude } \\
\text { (degrees, } \\
\text { minutes, } \\
\text { seconds) }\end{array}$ & $\begin{array}{c}\text { Longitude } \\
\text { (degrees, } \\
\text { minutes, } \\
\text { seconds) }\end{array}$ & $\begin{array}{c}\text { Source } \\
\text { of } \\
\text { data }\end{array}$ & $\begin{array}{c}\text { Depth } \\
\text { of well } \\
\text { (feet) }\end{array}$ & $\begin{array}{l}\text { Land-surface } \\
\text { datum altitude } \\
\text { (feet above } \\
\text { NGVD of } \\
\text { 1929) }\end{array}$ & $\begin{array}{c}\text { Depth to } \\
\text { water } \\
\text { (feet below } \\
\text { land-surface } \\
\text { datum) }\end{array}$ & $\begin{array}{l}\text { Water-level } \\
\text { altitude } \\
\text { (feet above } \\
\text { NGVD of } \\
\text { 1929) }\end{array}$ & $\begin{array}{c}\text { Date of } \\
\text { measure- } \\
\text { ment }\end{array}$ \\
\hline \multicolumn{9}{|c|}{ Mississippi County_Continued } \\
\hline 12N08E08BCB1 & 354047 & 901559 & USGS & 120 & 225 & 10.07 & 215 & $4 / 20 / 2006$ \\
\hline 12N08E28DDB1 & 353707 & 901406 & NRCS & 120 & 225 & 20 & 205 & $4 / 18 / 2006$ \\
\hline $12 \mathrm{~N} 09 \mathrm{E} 12 \mathrm{ABC} 1$ & 354054 & 900449 & NRCS & 120 & 232 & 17 & 215 & $4 / 14 / 2006$ \\
\hline 12N10E04CAA1 & 354124 & 900136 & NRCS & 120 & 235 & 20 & 215 & $4 / 14 / 2006$ \\
\hline 12N10E07BCD1 & 354036 & 900404 & NRCS & 110 & 234 & 22 & 212 & $4 / 14 / 2006$ \\
\hline 12N10E21DBA1 & 353842 & 900122 & NRCS & 110 & 236 & 17 & 219 & $4 / 14 / 2006$ \\
\hline 13N08E24ABB1 & 354428 & 901112 & NRCS & 120 & 230 & 9 & 221 & $4 / 18 / 2006$ \\
\hline 13N09E30CCD1 & 354248 & 901029 & USGS & -- & 230 & 12.88 & 217 & $4 / 20 / 2006$ \\
\hline 13N10E34DBB1 & 354218 & 900024 & USGS & 98 & 235 & 8.58 & 226 & $4 / 20 / 2006$ \\
\hline 14N08E12DAB1 & 355104 & 901052 & USGS & -- & 235 & 8.38 & 227 & $4 / 19 / 2006$ \\
\hline 14N08E20DAA1 & 354921 & 901458 & NRCS & 110 & 225 & 5 & 220 & $4 / 18 / 2006$ \\
\hline 14N08E26CC1 & 354803 & 901235 & NRCS & 100 & 230 & 5 & 225 & $4 / 18 / 2006$ \\
\hline 14N10E18ABC1 & 355022 & 900345 & USGS & 101 & 236 & 13.02 & 223 & $4 / 19 / 2006$ \\
\hline 14N11E03BCB1 & 355158 & 895433 & USGS & 128 & 247 & 5.26 & 242 & $4 / 20 / 2006$ \\
\hline 14N11E17CCB1 & 354955 & 895639 & NRCS & 120 & 240 & 8 & 232 & $4 / 10 / 2006$ \\
\hline 14N11E33CAA1 & 354727 & 895508 & NRCS & 120 & 240 & 15 & 225 & $4 / 10 / 2006$ \\
\hline $15 \mathrm{~N} 08 \mathrm{E} 08 \mathrm{DBC} 2$ & 355605 & 901526 & USGS & 120 & 236 & 11.97 & 224 & $4 / 19 / 2006$ \\
\hline 15N10E21ABC1 & 355447 & 900135 & NRCS & 120 & 240 & 13 & 227 & $4 / 13 / 2006$ \\
\hline 15N12E01BCD1 & 355704 & 894601 & NRCS & 100 & 258 & 11 & 247 & $4 / 13 / 2006$ \\
\hline 16N10E28BBD1* & 355906 & 900156 & NRCS & 120 & 238 & 14.5 & 224 & $4 / 13 / 2006$ \\
\hline 16N10E28BBD1* & 355906 & 900156 & USGS & 120 & 238 & 11.35 & 227 & $4 / 20 / 2006$ \\
\hline 16N11E23ADA1 & 355947 & 895231 & USGS & & 255 & 12.70 & 242 & $4 / 20 / 2006$ \\
\hline \multicolumn{9}{|c|}{ Monroe County } \\
\hline 01N01W21CDC2 & 344037 & 910707 & USGS & 150 & 181 & 36.57 & 144 & $4 / 04 / 2006$ \\
\hline 01N02W12CBC1 & 344242 & 911032 & USGS & 110 & 182 & 39.21 & 143 & $4 / 04 / 2006$ \\
\hline 01N03W23BAC1 & 344124 & 911743 & NRCS & 100 & 170 & 16 & 154 & $4 / 14 / 2006$ \\
\hline 01N03W24BBB1 & 344135 & 911651 & USGS & 125 & 185 & 28.63 & 156 & $4 / 04 / 2006$ \\
\hline 01N04W33BBB2 & 343960 & 912649 & USGS & -- & 218 & 95.15 & 123 & $4 / 04 / 2006$ \\
\hline 01S01W13CDD1 & 343611 & 910341 & USGS & 135 & 178 & 20.37 & 158 & $4 / 04 / 2006$ \\
\hline 01S01W16DB & 343615 & 910632 & NRCS & 100 & 175 & 22 & 153 & $4 / 14 / 2006$ \\
\hline 01S01W18DCD1 & 343618 & 910849 & USGS & 110 & 178 & 23.05 & 155 & $4 / 04 / 2006$ \\
\hline 01S02W20BBB $1 *$ & 343613 & 911456 & USGS & 100 & 170 & 13.35 & 157 & $4 / 04 / 2006$ \\
\hline 01S02W20BBB $1 *$ & 343613 & 911456 & NRCS & 100 & 170 & 12 & 158 & $4 / 14 / 2006$ \\
\hline 01S03W20BBA1* & 343538 & 912118 & USGS & 140 & 210 & 73.73 & 136 & $4 / 04 / 2006$ \\
\hline
\end{tabular}


Appendix 1. Information pertaining to water levels measured in wells completed in the Mississippi River Valley alluvial aquifer in eastern Arkansas, spring 2006.-Continued

[USGS, U.S. Geological Survey; NRCS, Natural Resources Conservation Service; --, no data; NGVD of 1929, National Geodetic Vertical Datum of 1929; Horizontal coordinate information is referenced to the North American Datum of 1983 (NAD 83); *, control wells for duplicate measurements by USGS and NRCS]

\begin{tabular}{|c|c|c|c|c|c|c|c|c|}
\hline Station name & $\begin{array}{c}\text { Latitude } \\
\text { (degrees, } \\
\text { minutes, } \\
\text { seconds) }\end{array}$ & $\begin{array}{l}\text { Longitude } \\
\text { (degrees, } \\
\text { minutes, } \\
\text { seconds) }\end{array}$ & $\begin{array}{c}\text { Source } \\
\text { of } \\
\text { data }\end{array}$ & $\begin{array}{c}\text { Depth } \\
\text { of well } \\
\text { (feet) }\end{array}$ & $\begin{array}{l}\text { Land-surface } \\
\text { datum altitude } \\
\text { (feet above } \\
\text { NGVD of } \\
\text { 1929) }\end{array}$ & $\begin{array}{c}\text { Depth to } \\
\text { water } \\
\text { (feet below } \\
\text { land-surface } \\
\text { datum) }\end{array}$ & $\begin{array}{c}\text { Water-level } \\
\text { altitude } \\
\text { (feet above } \\
\text { NGVD of } \\
\text { 1929) }\end{array}$ & $\begin{array}{c}\text { Date of } \\
\text { measure } \\
\text { ment }\end{array}$ \\
\hline \multicolumn{9}{|c|}{ Monroe County-Continued } \\
\hline 01S03W20BBA1* & 343538 & 912118 & NRCS & 140 & 210 & 79 & 131 & 4/14/2006 \\
\hline 01S04W01BAB1 & 343906 & 912317 & USGS & 160 & 210 & 68.70 & 141 & $4 / 04 / 2006$ \\
\hline 02N01W19ADD1 & 344624 & 910814 & NRCS & 80 & 188 & 53 & 135 & $4 / 14 / 2006$ \\
\hline 02N01W19BBA1 & 344645 & 910912 & USGS & 75 & 191 & 54.27 & 137 & $4 / 05 / 2006$ \\
\hline 02N03W35BCA1 & 344455 & 911745 & NRCS & 100 & 188 & 34 & 154 & $4 / 14 / 2006$ \\
\hline 02S01W01BCD1 & 343305 & 910408 & NRCS & 100 & 176 & 19 & 157 & $4 / 14 / 2006$ \\
\hline 02S02W01BCA1 & 343322 & 911031 & USGS & -- & 171 & 12.13 & 159 & 4/04/2006 \\
\hline 02S02W11DAC1 & 343209 & 911101 & USGS & 110 & 164 & 10.16 & 154 & $4 / 04 / 2006$ \\
\hline 03N01W20ABA1 & 345201 & 910723 & USGS & -- & 189 & 48.07 & 141 & $4 / 05 / 2006$ \\
\hline 03N02W31 ADC1 & 344958 & 911447 & USGS & 95 & 190 & 38.53 & 151 & $4 / 05 / 2006$ \\
\hline 03N03W36AAA1 & 345027 & 911547 & USGS & 120 & 176 & 22.20 & 154 & 4/05/2006 \\
\hline 04N02W01BCC1 & 345929 & 911004 & NRCS & 100 & 175 & 39.5 & 136 & 4/13/2006 \\
\hline 04N02W05BBB1 & 345957 & 911311 & NRCS & 100 & 188 & 15 & 173 & $4 / 13 / 2006$ \\
\hline 04N02W27CDD3 & 345540 & 911150 & USGS & 181 & 200 & 45.50 & 155 & $4 / 05 / 2006$ \\
\hline 04N02W28DDD3 & 345535 & 911221 & USGS & 137 & 192 & 32.19 & 160 & $4 / 05 / 2006$ \\
\hline 04N02W30BBB1 & 345628 & 911525 & USGS & 119 & 185.16 & 14.53 & 171 & 4/05/2006 \\
\hline \multicolumn{9}{|c|}{ Phillips County } \\
\hline 01S01E20DDB1 & 343529 & 910058 & NRCS & 114 & 185 & 26.0 & 159 & $4 / 10 / 2006$ \\
\hline 01S02E09CBB1* & 343719 & 905434 & USGS & 110 & 185 & 14.29 & 171 & $3 / 21 / 2006$ \\
\hline 01S02E09CBB1* & 343719 & 905434 & NRCS & 110 & 185 & 14.8 & 170 & $4 / 10 / 2006$ \\
\hline 01S02E32BCC1 & 343350 & 905526 & NRCS & 120 & 200 & 37.0 & 163 & $4 / 10 / 2006$ \\
\hline 01S03E02ADD1 & 343814 & 904511 & NRCS & 120 & 200 & 16.6 & 183 & $4 / 10 / 2006$ \\
\hline 01S03E10ABB1 & 343741 & 904634 & NRCS & 120 & 205 & 18.0 & 187 & $4 / 10 / 2006$ \\
\hline 01S03E20BDD1 & 343533 & 904846 & NRCS & 120 & 210 & 33.0 & 177 & $4 / 10 / 2006$ \\
\hline 01S04E05DCD1* & 343802 & 904151 & USGS & 120 & 230 & 48.91 & 181 & $3 / 21 / 2006$ \\
\hline 01S04E05DCD1* & 343802 & 904151 & NRCS & 120 & 230 & 49.0 & 181 & $4 / 10 / 2006$ \\
\hline 02S01E28CCB1 & 342916 & 910058 & USGS & 108 & 174 & 17.95 & 156 & $3 / 21 / 2006$ \\
\hline 02S02E29DDD1 & 342901 & 905444 & NRCS & 125 & 180 & 27.6 & 152 & $4 / 10 / 2006$ \\
\hline 02S02E33ACC1 & 342824 & 905412 & NRCS & 120 & 177 & 26.0 & 151 & $4 / 10 / 2006$ \\
\hline 02S03E15ACD1 & 343110 & 904621 & USGS & 112 & 174 & 13.78 & 160 & $5 / 08 / 2006$ \\
\hline 02S03E34BCD1 & 342828 & 904653 & NRCS & 120 & 165 & 18.0 & 147 & $4 / 12 / 2006$ \\
\hline 02S04E27AAC1* & 342932 & 904001 & USGS & 175 & 179 & 8.59 & 170 & $3 / 21 / 2006$ \\
\hline 02S04E27AAC1* & 342932 & 904001 & NRCS & 175 & 179 & 10.0 & 169 & $4 / 10 / 2006$ \\
\hline 03S02E35DDA1 & 342256 & 905130 & USGS & 50 & 163 & 21.23 & 142 & $3 / 21 / 2006$ \\
\hline
\end{tabular}


Appendix 1. Information pertaining to water levels measured in wells completed in the Mississippi River Valley alluvial aquifer in eastern Arkansas, spring 2006.-Continued

[USGS, U.S. Geological Survey; NRCS, Natural Resources Conservation Service; --, no data; NGVD of 1929, National Geodetic Vertical Datum of 1929; Horizontal coordinate information is referenced to the North American Datum of 1983 (NAD 83); *, control wells for duplicate measurements by USGS and NRCS]

\begin{tabular}{|c|c|c|c|c|c|c|c|c|}
\hline Station name & $\begin{array}{c}\text { Latitude } \\
\text { (degrees, } \\
\text { minutes, } \\
\text { seconds) }\end{array}$ & $\begin{array}{c}\text { Longitude } \\
\text { (degrees, } \\
\text { minutes, } \\
\text { seconds) }\end{array}$ & $\begin{array}{c}\text { Source } \\
\text { of } \\
\text { data }\end{array}$ & $\begin{array}{c}\text { Depth } \\
\text { of well } \\
\text { (feet) }\end{array}$ & $\begin{array}{l}\text { Land-surface } \\
\text { datum altitude } \\
\text { (feet above } \\
\text { NGVD of } \\
\text { 1929) }\end{array}$ & $\begin{array}{c}\text { Depth to } \\
\text { water } \\
\text { (feet below } \\
\text { land-surface } \\
\text { datum) }\end{array}$ & $\begin{array}{l}\text { Water-level } \\
\text { altitude } \\
\text { (feet above } \\
\text { NGVD of } \\
\text { 1929) }\end{array}$ & $\begin{array}{c}\text { Date of } \\
\text { measure- } \\
\text { ment }\end{array}$ \\
\hline \multicolumn{9}{|c|}{ Phillips County-Continued } \\
\hline 03S03E04DAA1 & 342735 & 904710 & USGS & 36 & 171 & 19.29 & 152 & $3 / 21 / 2006$ \\
\hline 03S04E02CAA1* & 342732 & 903918 & USGS & 120 & 176 & 16.16 & 160 & $3 / 21 / 2006$ \\
\hline 03S04E02CAA1* & 342732 & 903918 & NRCS & 120 & 176 & 17.0 & 159 & $4 / 10 / 2006$ \\
\hline 04S01E01AAD1 & 342238 & 905700 & NRCS & 120 & 156 & 22.0 & 134 & $4 / 12 / 2006$ \\
\hline 04S01E14CDD1 & 342014 & 905837 & NRCS & 120 & 155 & 15.0 & 140 & $4 / 12 / 2006$ \\
\hline 04S01E23CCA1 & 341931 & 905853 & USGS & -- & 156 & 13.4 & 143 & $3 / 21 / 2006$ \\
\hline 04S02E01DBB1 & 342220 & 905053 & NRCS & -- & 163 & 15.6 & 147 & $4 / 12 / 2006$ \\
\hline 05S02E18BDA1 & 341535 & 905628 & USGS & 130 & 156 & 22.5 & 134 & $3 / 21 / 2006$ \\
\hline \multicolumn{9}{|c|}{ Poinsett County } \\
\hline 10N01E02AAA & 353205 & 905654 & NRCS & 100 & 235 & 98 & 137 & $4 / 13 / 2006$ \\
\hline 10N01E14CC1 & 352910 & 905814 & USGS & 150 & 231 & 92.41 & 139 & $3 / 28 / 2006$ \\
\hline 10N01E16CCB1 & 352922 & 910005 & USGS & 120 & 225 & 74.99 & 150 & $3 / 28 / 2006$ \\
\hline 10N01E32CBB1 & 352657 & 910053 & NRCS & 120 & 222 & 74 & 148 & $4 / 13 / 2006$ \\
\hline 10N01E33ACB1 & 352746 & 905931 & NRCS & 153 & 220 & 77 & 143 & $4 / 13 / 2006$ \\
\hline 10N02E13BCC1 & 352949 & 905026 & USGS & 167 & 237 & 103.24 & 134 & $3 / 28 / 2006$ \\
\hline 10N02E15CAA1 & 352940 & 905209 & NRCS & 160 & 237 & 104.5 & 133 & $4 / 12 / 2006$ \\
\hline 10N02E20BAB1 & 352906 & 905418 & NRCS & 155 & 237 & 112.5 & 125 & $4 / 13 / 2006$ \\
\hline 10N03E13BCB1 & 352958 & 904352 & NRCS & 155 & 275 & 142 & 133 & $4 / 13 / 2006$ \\
\hline 10N03E14DAB1 & 352947 & 904405 & USGS & -- & 263 & 118.63 & 144 & $3 / 28 / 2006$ \\
\hline 10N03E19BCB1 & 352905 & 904907 & NRCS & -- & 239 & 99.5 & 140 & $4 / 12 / 2006$ \\
\hline 10N03E26BBD1 & 352816 & 904449 & NRCS & 140 & 257 & 113.5 & 144 & $4 / 13 / 2006$ \\
\hline 10N03E35CDD1 & 352656 & 904436 & USGS & -- & 275 & 124.43 & 151 & $3 / 28 / 2006$ \\
\hline 10N04E35BBA1 & 352745 & 903831 & NRCS & 100 & 212 & 21 & 191 & $4 / 17 / 2006$ \\
\hline 10N05E15BDD1 & 352937 & 903253 & USGS & -- & 207 & 14.76 & 192 & $3 / 29 / 2006$ \\
\hline 10N06E28ABC1 & 352804 & 902723 & NRCS & 150 & 210 & 20 & 190 & $4 / 17 / 2006$ \\
\hline 10N07E22AAC1 & 352847 & 901935 & USGS & -- & 215 & 27.80 & 187 & $3 / 29 / 2006$ \\
\hline 10N07E28CBB1 & 352733 & 902128 & NRCS & 105 & 217 & 28.5 & 189 & $4 / 17 / 2006$ \\
\hline 11N01E17DDC1 & 353437 & 910015 & NRCS & 100 & 232 & 78 & 154 & $4 / 13 / 2006$ \\
\hline 11N01E17DDD1 & 353437 & 910013 & USGS & 100 & 230 & 78.61 & 151 & $3 / 28 / 2006$ \\
\hline 11N01E26AA1 & 353340 & 905653 & USGS & 140 & 236 & 94.72 & 141 & $3 / 28 / 2006$ \\
\hline 11N01E34AAA & 353256 & 905759 & NRCS & 100 & 229 & 88.5 & 141 & $4 / 13 / 2006$ \\
\hline 11N02E26AAB1 & 353350 & 905034 & USGS & 158 & 241 & 107.55 & 133 & $3 / 28 / 2006$ \\
\hline 11N02E30BBB1 & 353352 & 905540 & NRCS & 140 & 239 & 102.5 & 137 & $4 / 17 / 2006$ \\
\hline 11N02E34CBA1 & 353238 & 905222 & NRCS & 130 & 240 & 109 & 131 & $4 / 13 / 2006$ \\
\hline
\end{tabular}


Appendix 1. Information pertaining to water levels measured in wells completed in the Mississippi River Valley alluvial aquifer in eastern Arkansas, spring 2006.-Continued

[USGS, U.S. Geological Survey; NRCS, Natural Resources Conservation Service; --, no data; NGVD of 1929, National Geodetic Vertical Datum of 1929; Horizontal coordinate information is referenced to the North American Datum of 1983 (NAD 83); *, control wells for duplicate measurements by USGS and NRCS]

\begin{tabular}{|c|c|c|c|c|c|c|c|c|}
\hline Station name & $\begin{array}{l}\text { Latitude } \\
\text { (degrees, } \\
\text { minutes, } \\
\text { seconds) }\end{array}$ & $\begin{array}{c}\text { Longitude } \\
\text { (degrees, } \\
\text { minutes, } \\
\text { seconds) }\end{array}$ & $\begin{array}{c}\text { Source } \\
\text { of } \\
\text { data }\end{array}$ & $\begin{array}{c}\text { Depth } \\
\text { of well } \\
\text { (feet) }\end{array}$ & $\begin{array}{l}\text { Land-surface } \\
\text { datum altitude } \\
\text { (feet above } \\
\text { NGVD of } \\
\text { 1929) }\end{array}$ & $\begin{array}{c}\text { Depth to } \\
\text { water } \\
\text { (feet below } \\
\text { land-surface } \\
\text { datum) }\end{array}$ & $\begin{array}{l}\text { Water-level } \\
\text { altitude } \\
\text { (feet above } \\
\text { NGVD of } \\
\text { 1929) }\end{array}$ & $\begin{array}{c}\text { Date of } \\
\text { measure- } \\
\text { ment }\end{array}$ \\
\hline \multicolumn{9}{|c|}{ Poinsett County-Continued } \\
\hline 11N03E10DDA1 & 353546 & 904457 & USGS & 145 & 243 & 104.78 & 138 & $3 / 28 / 2006$ \\
\hline 11N03E17AAB1 & 353535 & 904714 & NRCS & -- & 242 & 105 & 137 & $4 / 17 / 2006$ \\
\hline 11N03E18BAB1 & 353538 & 904852 & USGS & 157 & 243 & 105.34 & 138 & $3 / 28 / 2006$ \\
\hline 11N04E36ABA1 & 353251 & 903654 & NRCS & 100 & 211 & 18 & 193 & $4 / 17 / 2006$ \\
\hline 11N05E26BDB1 & 353318 & 903213 & NRCS & -- & 213 & 12 & 201 & $4 / 17 / 2006$ \\
\hline 11N07E18CAB1 & 353435 & 902320 & USGS & 100 & 217 & 15.37 & 202 & $3 / 29 / 2006$ \\
\hline 11W04E13DDA1 & 353445 & 903632 & NRCS & -- & 211 & 15 & 196 & $4 / 17 / 2006$ \\
\hline 12N01E07CDA1 & 354054 & 910141 & USGS & 120 & 236 & 53.97 & 182 & $3 / 28 / 2006$ \\
\hline 12N01E22DAB1 & 353922 & 905809 & NRCS & 115 & 235 & 74 & 161 & $4 / 13 / 2006$ \\
\hline 12N02E25DCC1 & 353820 & 904944 & NRCS & 145 & 245 & 112 & 133 & $4 / 13 / 2006$ \\
\hline $12 \mathrm{~N} 02 \mathrm{E} 34 \mathrm{CCC} 1$ & 353724 & 905230 & NRCS & 180 & 245 & 112.5 & 133 & $4 / 13 / 2006$ \\
\hline 12N03E01CBD1 & 354154 & 904329 & NRCS & 190 & 250 & 93 & 157 & $4 / 17 / 2006$ \\
\hline 12N03E04DAD1* & 354158 & 904600 & USGS & 120 & 247 & 103.52 & 143 & $3 / 28 / 2006$ \\
\hline 12N03E04DAD1* & 354158 & 904600 & NRCS & 120 & 247 & 104 & 143 & $4 / 17 / 2006$ \\
\hline 12N03E35ADD1 & 353745 & 904352 & NRCS & 160 & 246 & 103 & 143 & $4 / 17 / 2006$ \\
\hline 12N03E36ACB1 & 353749 & 904319 & USGS & 120 & 250 & 98.57 & 151 & $3 / 28 / 2006$ \\
\hline 12N04E08CDA & 354053 & 904112 & NRCS & 100 & 250 & 88 & 162 & $4 / 17 / 2006$ \\
\hline 12N05E16ABA1 & 354039 & 903333 & NRCS & 140 & 221 & 12 & 209 & $4 / 17 / 2006$ \\
\hline 12N05E34ABA1 & 353805 & 903230 & USGS & 100 & 215 & 10.65 & 204 & $3 / 28 / 2006$ \\
\hline 12N07E04BAA1 & 354202 & 902060 & USGS & 60 & 223 & 6.93 & 216 & $3 / 29 / 2006$ \\
\hline 12N07E10CBB1 & 354042 & 902022 & NRCS & 100 & 220 & 10 & 210 & $4 / 17 / 2006$ \\
\hline \multicolumn{9}{|c|}{ Prairie County } \\
\hline 01N06W05CCB1 & 344353 & 914049 & USGS & 155 & 220 & 117.80 & 102 & $4 / 11 / 2006$ \\
\hline 01N06W29DDD1 & 344018 & 913951 & USGS & 155 & 235 & 116.55 & 118 & $4 / 11 / 2006$ \\
\hline 01S04W28BDB1 & 343523 & 912630 & USGS & 112 & 205 & 97.28 & 108 & $4 / 11 / 2006$ \\
\hline 01S05W14BBC1 & 343722 & 913109 & USGS & 118 & 211 & 108.15 & 103 & $4 / 11 / 2006$ \\
\hline 01S05W31DDA1 & 343417 & 913432 & USGS & 120 & 206 & 103.60 & 102 & $4 / 11 / 2006$ \\
\hline 02N04W02BCB1 & 344916 & 912419 & USGS & 140 & 188 & 20.27 & 168 & $4 / 11 / 2006$ \\
\hline 02N04W32CCB1 & 344436 & 912738 & USGS & -- & 221 & 84.72 & 136 & $4 / 11 / 2006$ \\
\hline 02N05W06BAB1 & 344958 & 913421 & USGS & 145 & 221 & 89.19 & 132 & $4 / 11 / 2006$ \\
\hline 02N05W13AAB1 & 344805 & 912854 & USGS & 130 & 223 & 81.74 & 141 & $4 / 11 / 2006$ \\
\hline 02N05W29DDB2 & 344545 & 913309 & USGS & 135 & 228 & 118.84 & 109 & $4 / 11 / 2006$ \\
\hline 02N06W17ABB1 & 344809 & 913959 & USGS & 180 & 235 & 124.26 & 111 & $4 / 11 / 2006$ \\
\hline 02S06W14BBB1 & 343213 & 913729 & USGS & 105 & 201 & 74.29 & 127 & $4 / 11 / 2006$ \\
\hline
\end{tabular}


Appendix 1. Information pertaining to water levels measured in wells completed in the Mississippi River Valley alluvial aquifer in eastern Arkansas, spring 2006.-Continued

[USGS, U.S. Geological Survey; NRCS, Natural Resources Conservation Service; --, no data; NGVD of 1929, National Geodetic Vertical Datum of 1929; Horizontal coordinate information is referenced to the North American Datum of 1983 (NAD 83); *, control wells for duplicate measurements by USGS and NRCS]

\begin{tabular}{|c|c|c|c|c|c|c|c|c|}
\hline Station name & $\begin{array}{c}\text { Latitude } \\
\text { (degrees, } \\
\text { minutes, } \\
\text { seconds) }\end{array}$ & $\begin{array}{c}\text { Longitude } \\
\text { (degrees, } \\
\text { minutes, } \\
\text { seconds) }\end{array}$ & $\begin{array}{c}\text { Source } \\
\text { of } \\
\text { data }\end{array}$ & $\begin{array}{c}\text { Depth } \\
\text { of well } \\
\text { (feet) }\end{array}$ & $\begin{array}{l}\text { Land-surface } \\
\text { datum altitude } \\
\text { (feet above } \\
\text { NGVD of } \\
\text { 1929) }\end{array}$ & $\begin{array}{c}\text { Depth to } \\
\text { water } \\
\text { (feet below } \\
\text { land-surface } \\
\text { datum) }\end{array}$ & $\begin{array}{l}\text { Water-level } \\
\text { altitude } \\
\text { (feet above } \\
\text { NGVD of } \\
\text { 1929) }\end{array}$ & $\begin{array}{c}\text { Date of } \\
\text { measure- } \\
\text { ment }\end{array}$ \\
\hline \multicolumn{9}{|c|}{ Prairie County-Continued } \\
\hline 03N04W03AAC1 & 345439 & 912424 & USGS & 106 & 187 & 28.30 & 159 & $4 / 11 / 2006$ \\
\hline 03N05W03BDD2 & 345444 & 913115 & USGS & 110 & 207 & 67.70 & 139 & $4 / 11 / 2006$ \\
\hline 03N06W01BCB1 & 345455 & 913601 & USGS & 115 & 216 & 79.08 & 137 & $4 / 11 / 2006$ \\
\hline 03N06W19BDD1 & 345207 & 914110 & USGS & 105 & 221 & 86.73 & 134 & $4 / 12 / 2006$ \\
\hline 04N04W07ADC1 & 345850 & 912733 & USGS & 110 & 195 & 26.73 & 168 & $4 / 11 / 2006$ \\
\hline 04N05W07CDC1 & 345043 & 913441 & USGS & -- & 212 & 76.54 & 135 & $4 / 12 / 2006$ \\
\hline 04N06W05CCC1 & 345934 & 914018 & USGS & 100 & 206 & 78.35 & 128 & $4 / 11 / 2006$ \\
\hline 04N07W03DCB1 & 345942 & 914412 & USGS & 100 & 255 & 87.33 & 168 & $4 / 12 / 2006$ \\
\hline 04N07W28BBA1 & 345701 & 914545 & USGS & 110 & 258 & 95.77 & 162 & $4 / 12 / 2006$ \\
\hline 05N05W14DCD1 & 350252 & 913034 & USGS & -- & 205 & 39.62 & 165 & $4 / 12 / 2006$ \\
\hline 05N05W28DDA1 & 350119 & 913228 & NRCS & 85 & 191 & 63.0 & 128 & $4 / 23 / 2006$ \\
\hline \multicolumn{9}{|c|}{ Pulaski County } \\
\hline 01S10W29CC1 & 343538 & 920708 & USGS & 100 & 239 & 18.02 & 221 & $4 / 03 / 2006$ \\
\hline 02S10W14DC1 & 343205 & 920334 & USGS & 60 & 225 & 24.90 & 200 & $4 / 03 / 2006$ \\
\hline 02S10W16CCA1 & 343217 & 920549 & USGS & & 230.76 & 27.27 & 203 & $4 / 03 / 2006$ \\
\hline \multicolumn{9}{|c|}{ Randolph County } \\
\hline 18N01E28AAD1 & 361040 & 905820 & NRCS & 120 & 265 & 22 & 243 & $4 / 24 / 2006$ \\
\hline 18N01E34AAC1 & 360943 & 905729 & USGS & -- & 266 & 18.18 & 248 & $4 / 17 / 2006$ \\
\hline 18N02E03DAD1 & 361336 & 905043 & NRCS & 120 & 280 & 56 & 224 & $4 / 24 / 2006$ \\
\hline 18N02E17CBB1 & 361204 & 905356 & NRCS & -- & 265 & 23 & 242 & $4 / 24 / 2006$ \\
\hline 18N02E20BDA1 & 361125 & 905332 & NRCS & 110 & 274 & 42.5 & 232 & $4 / 24 / 2006$ \\
\hline 18N02E22DCD1 & 361046 & 905105 & USGS & 110 & 273 & 37.47 & 236 & $4 / 17 / 2006$ \\
\hline 18N02E34BCC1 & 360933 & 905150 & NRCS & 100 & 265 & 31 & 234 & $4 / 24 / 2006$ \\
\hline 19N02E09ABD1* & 361826 & 905157 & USGS & 80 & 266 & 11.23 & 255 & $4 / 17 / 2006$ \\
\hline 19N02E09ABD1* & 361826 & 905157 & NRCS & 80 & 266 & 18 & 248 & $4 / 25 / 2006$ \\
\hline 19N02E22DAB1 & 361622 & 905049 & NRCS & 90 & 266 & 15.5 & 251 & $4 / 24 / 2006$ \\
\hline 20N02E01ADD1 & 362424 & 904811 & USGS & 65 & 280 & 13.93 & 266 & $4 / 17 / 2006$ \\
\hline 20N02E12BAA1 & 362352 & 904848 & NRCS & 60 & 281 & 12 & 269 & $4 / 25 / 2006$ \\
\hline 20N02E14DAB1 & 362232 & 904930 & NRCS & 100 & 274 & 14 & 260 & $4 / 25 / 2006$ \\
\hline 20N02E21CDD1 & 362117 & 905107 & NRCS & 110 & 270 & 12 & 258 & $4 / 25 / 2006$ \\
\hline 20N03E28BA1 & 362114 & 904538 & USGS & -- & 276 & 12.86 & 263 & $4 / 17 / 2006$ \\
\hline 20N03E33CCA1 & 361941 & 904552 & NRCS & -- & 287 & 26 & 261 & $4 / 24 / 2006$ \\
\hline \multicolumn{9}{|c|}{ St. Francis County } \\
\hline 04N01E13ADA1 & 345755 & 905638 & USGS & -- & 206 & 60.34 & 146 & $3 / 22 / 2006$ \\
\hline
\end{tabular}


Appendix 1. Information pertaining to water levels measured in wells completed in the Mississippi River Valley alluvial aquifer in eastern Arkansas, spring 2006.-Continued

[USGS, U.S. Geological Survey; NRCS, Natural Resources Conservation Service; --, no data; NGVD of 1929, National Geodetic Vertical Datum of 1929; Horizontal coordinate information is referenced to the North American Datum of 1983 (NAD 83); *, control wells for duplicate measurements by USGS and NRCS]

\begin{tabular}{|c|c|c|c|c|c|c|c|c|}
\hline Station name & $\begin{array}{c}\text { Latitude } \\
\text { (degrees, } \\
\text { minutes, } \\
\text { seconds) }\end{array}$ & $\begin{array}{c}\text { Longitude } \\
\text { (degrees, } \\
\text { minutes, } \\
\text { seconds) }\end{array}$ & $\begin{array}{c}\text { Source } \\
\text { of } \\
\text { data }\end{array}$ & $\begin{array}{c}\text { Depth } \\
\text { of well } \\
\text { (feet) }\end{array}$ & $\begin{array}{l}\text { Land-surface } \\
\text { datum altitude } \\
\text { (feet above } \\
\text { NGVD of } \\
\text { 1929) }\end{array}$ & $\begin{array}{c}\text { Depth to } \\
\text { water } \\
\text { (feet below } \\
\text { land-surface } \\
\text { datum) }\end{array}$ & $\begin{array}{l}\text { Water-level } \\
\text { altitude } \\
\text { (feet above } \\
\text { NGVD of } \\
\text { 1929) }\end{array}$ & $\begin{array}{c}\text { Date of } \\
\text { measure- } \\
\text { ment }\end{array}$ \\
\hline \multicolumn{9}{|c|}{ St. Francis County-Continued } \\
\hline 04N01W20BBB1 & 345716 & 910759 & NRCS & 140 & 200 & 59 & 141 & $5 / 15 / 2006$ \\
\hline 04N01W25DBD1 & 345549 & 910303 & NRCS & 140 & 199 & 76 & 123 & $5 / 15 / 2006$ \\
\hline 04N01W28CDD1 & 345535 & 910634 & USGS & -- & 208 & 71.42 & 137 & $3 / 22 / 2006$ \\
\hline 04N02E16ACD1 & 345733 & 905341 & NRCS & 140 & 209 & 41 & 168 & $5 / 15 / 2006$ \\
\hline 04N02E19BBB1 & 345701 & 905633 & USGS & 72.2 & 209 & 60.16 & 149 & $3 / 22 / 2006$ \\
\hline 04N02E27AAA1 & 345604 & 905220 & NRCS & 140 & 211 & 48 & 163 & $5 / 15 / 2006$ \\
\hline 04N03E21DAD1 & 345623 & 904655 & USGS & -- & 236 & 58.14 & 178 & $3 / 22 / 2006$ \\
\hline 04N04E15ABA1 & 345752 & 903948 & NRCS & 120 & 201 & 34 & 167 & $5 / 15 / 2006$ \\
\hline 04N05E22BBB1 & 345651 & 903357 & USGS & -- & 200 & 27.02 & 173 & $3 / 22 / 2006$ \\
\hline 05N01E06CDA1 & 350437 & 910218 & NRCS & -- & 211 & 71 & 140 & $5 / 15 / 2006$ \\
\hline 05N01E15BCB1 & 350303 & 905942 & USGS & 94.1 & 209 & 67.19 & 142 & $3 / 22 / 2006$ \\
\hline 05N01E27BBA1 & 350136 & 905929 & USGS & -- & 209 & 66.93 & 142 & $3 / 22 / 2006$ \\
\hline 05N02E20ADC1 & 350157 & 905437 & USGS & 79 & 211 & 54.79 & 156 & $3 / 22 / 2006$ \\
\hline 05N03E20AAA2 & 350214 & 904801 & USGS & 153.45 & 250 & 103.87 & 146 & $3 / 22 / 2006$ \\
\hline 05N05E19DCA1 & 350128 & 903630 & USGS & 110 & 203 & 32.77 & 170 & $3 / 22 / 2006$ \\
\hline 05N05E33BCC1 & 350004 & 903506 & NRCS & 120 & 196 & 29 & 167 & $5 / 15 / 2006$ \\
\hline 05N06E34CAB1 & 350026 & 902657 & USGS & 110 & 200 & 27.69 & 172 & $3 / 22 / 2006$ \\
\hline 06N01E33ACA2 & 350552 & 905942 & USGS & -- & 211 & 67.14 & 144 & $3 / 22 / 2006$ \\
\hline 06N02E13DCA1 & 350813 & 905003 & USGS & -- & 231 & 73.85 & 157 & $3 / 22 / 2006$ \\
\hline 06N02E15BDD1 & 350842 & 905247 & USGS & 75 & 214.64 & 60.15 & 154 & $3 / 22 / 2006$ \\
\hline 06N02E16CCC1 & 350804 & 905403 & NRCS & 120 & 216 & 66.5 & 150 & $5 / 15 / 2006$ \\
\hline 06N02E24AAA1 & 350755 & 905002 & USGS & 147 & 232 & 71.46 & 161 & $3 / 22 / 2006$ \\
\hline 06N05E22ACC1 & 350723 & 903252 & USGS & -- & 200 & 40.63 & 159 & $3 / 22 / 2006$ \\
\hline 06N06E17DDC1 & 350749 & 902830 & NRCS & -- & 202 & 34 & 168 & $5 / 15 / 2006$ \\
\hline 06N06E20ABB2 & 350747 & 902841 & USGS & 150 & 200 & 36.75 & 163 & $3 / 22 / 2006$ \\
\hline \multicolumn{9}{|c|}{ White County } \\
\hline 05N07W09AAA1 & 350447 & 914441 & USGS & 29.5 & 205 & 14.32 & 191 & $3 / 23 / 2006$ \\
\hline 05N07W10CCC1 & 350400 & 914436 & USGS & 80 & 203 & 9.01 & 194 & $3 / 23 / 2006$ \\
\hline 06N06W04BAA1 & 351047 & 913910 & USGS & 70 & 220 & 35.47 & 185 & $3 / 23 / 2006$ \\
\hline 06N06W04BAD1 & 351037 & 913903 & NRCS & -- & 215 & 40.8 & 174 & $4 / 25 / 2006$ \\
\hline 06N06W13DBB1 & 350918 & 913552 & NRCS & -- & 213 & 47.3 & 166 & $4 / 25 / 2006$ \\
\hline 06N06W18BBC1 & 350851 & 914152 & USGS & -- & 210 & 18.13 & 192 & $3 / 23 / 2006$ \\
\hline 06N06W18BCA1 & 350835 & 914150 & NRCS & -- & 210 & 20.3 & 190 & $4 / 25 / 2006$ \\
\hline 06N06W34AAB1 & 350624 & 913754 & USGS & -- & 213 & 60.63 & 152 & $3 / 23 / 2006$ \\
\hline
\end{tabular}


Appendix 1. Information pertaining to water levels measured in wells completed in the Mississippi River Valley alluvial aquifer in eastern Arkansas, spring 2006.-Continued

[USGS, U.S. Geological Survey; NRCS, Natural Resources Conservation Service; --, no data; NGVD of 1929, National Geodetic Vertical Datum of 1929; Horizontal coordinate information is referenced to the North American Datum of 1983 (NAD 83); *, control wells for duplicate measurements by USGS and NRCS]

\begin{tabular}{|c|c|c|c|c|c|c|c|c|}
\hline Station name & $\begin{array}{c}\text { Latitude } \\
\text { (degrees, } \\
\text { minutes, } \\
\text { seconds) }\end{array}$ & $\begin{array}{l}\text { Longitude } \\
\text { (degrees, } \\
\text { minutes, } \\
\text { seconds) }\end{array}$ & $\begin{array}{c}\text { Source } \\
\text { of } \\
\text { data }\end{array}$ & $\begin{array}{l}\text { Depth } \\
\text { of well } \\
\text { (feet) }\end{array}$ & $\begin{array}{l}\text { Land-surface } \\
\text { datum altitude } \\
\text { (feet above } \\
\text { NGVD of } \\
\text { 1929) }\end{array}$ & $\begin{array}{c}\text { Depth to } \\
\text { water } \\
\text { (feet below } \\
\text { land-surface } \\
\text { datum) }\end{array}$ & $\begin{array}{c}\text { Water-level } \\
\text { altitude } \\
\text { (feet above } \\
\text { NGVD of } \\
\text { 1929) }\end{array}$ & $\begin{array}{c}\text { Date of } \\
\text { measure- } \\
\text { ment }\end{array}$ \\
\hline \multicolumn{9}{|c|}{ White County-Continued } \\
\hline 06N07W17DCC1 & 350822 & 914635 & USGS & 90 & 217 & 15.70 & 201 & $3 / 23 / 2006$ \\
\hline 06N08W13ABA1 & 350908 & 914824 & USGS & 60 & 228 & 13.60 & 214 & $3 / 23 / 2006$ \\
\hline 06N08W26DDB1 & 350640 & 914931 & USGS & 89 & 230 & 14.47 & 216 & $3 / 23 / 2006$ \\
\hline 07N05W01AAA1 & 351553 & 912858 & USGS & -- & 205 & 17.52 & 187 & $3 / 23 / 2006$ \\
\hline 07N05W32BAB1 & 351137 & 913406 & USGS & 80 & 213.7 & 32.64 & 181 & $3 / 23 / 2006$ \\
\hline 08N04W06CCB1 & 352028 & 912847 & USGS & 74 & 214 & 20.50 & 194 & $3 / 23 / 2006$ \\
\hline 08N05W32CBC1 & 351616 & 913417 & USGS & -- & 199 & 4.17 & 195 & $3 / 23 / 2006$ \\
\hline \multicolumn{9}{|c|}{ Woodruff County } \\
\hline 04N03W03AB1 & 350021 & 911820 & USGS & 100 & 185 & 14.61 & 170 & $3 / 28 / 2006$ \\
\hline 05N01W13CDC1 & 350244 & 910331 & NRCS & 135 & 210 & 74.6 & 135 & $4 / 05 / 2006$ \\
\hline 05N01W31CCC1 & 350106 & 910900 & NRCS & 140 & 210 & 59.1 & 151 & $4 / 05 / 2006$ \\
\hline 05N02W20DCB1 & 350208 & 911356 & USGS & -- & 192 & 15.25 & 177 & $3 / 28 / 2006$ \\
\hline 05N03W25DDB1 & 350133 & 911531 & NRCS & 120 & 190 & 12.7 & 177 & $4 / 05 / 2006$ \\
\hline 05N04W12DBA1 & 350427 & 912211 & USGS & 92 & 186 & 6.16 & 180 & $3 / 27 / 2006$ \\
\hline 06N01W06BAB1 & 351048 & 910835 & USGS & -- & 202 & 34.53 & 167 & $3 / 28 / 2006$ \\
\hline 06N01W11CBC1 & 350910 & 910542 & NRCS & 80 & 220 & 65.8 & 154 & $4 / 05 / 2006$ \\
\hline 06N02W19AAA1 & 350802 & 911419 & NRCS & 130 & 225 & 45.5 & 180 & $4 / 05 / 2006$ \\
\hline 06N03W15BAB1 & 350903 & 911807 & USGS & 111 & 188.79 & 6.63 & 182 & $3 / 27 / 2006$ \\
\hline 06N03W31BCB1 & 350623 & 912144 & USGS & -- & 185 & 2.86 & 182 & $3 / 27 / 2006$ \\
\hline 06N04W22BDA1 & 350807 & 912428 & NRCS & 120 & 186 & 5.9 & 180 & $4 / 05 / 2006$ \\
\hline 07N01W04ACB1 & 351541 & 910626 & NRCS & 125 & 225 & 60.9 & 164 & $4 / 05 / 2006$ \\
\hline 07N03W06BAC1 & 351607 & 912109 & NRCS & 100 & 211 & 24.4 & 187 & $4 / 05 / 2006$ \\
\hline 07N03W19AAA1 & 351335 & 912025 & USGS & 100 & 202.59 & 12.95 & 190 & $3 / 27 / 2006$ \\
\hline 07N03W31BBA1 & 351152 & 912103 & NRCS & 120 & 195 & 11.9 & 183 & $4 / 05 / 2006$ \\
\hline 08N01W06DDD1 & 352028 & 910747 & USGS & -- & 218 & 44.57 & 173 & $3 / 28 / 2006$ \\
\hline 08N02W27DDB1 & 351711 & 911107 & NRCS & 60 & 213 & 27 & 186 & 4/05/2006 \\
\hline 08N02W31DDD1 & 351611 & 911411 & USGS & 40 & 194.55 & 4.02 & 191 & $3 / 28 / 2006$ \\
\hline 08N03W31AAD1 & 351655 & 912028 & USGS & 110 & 212 & 21.95 & 190 & $3 / 27 / 2006$ \\
\hline 08N04W27AAA1 & 351757 & 912341 & USGS & -- & 200 & 13.10 & 187 & $3 / 27 / 2006$ \\
\hline 09N03W28ABB1 & 352310 & 911845 & NRCS & 120 & 220 & 19.6 & 200 & $4 / 05 / 2006$ \\
\hline 09N03W29AAD1 & 352258 & 911921 & USGS & -- & 220 & 22.53 & 197 & $3 / 27 / 2006$ \\
\hline 09N03W32ACA1 & 352205 & 911936 & NRCS & 120 & 217 & 21.6 & 195 & $4 / 05 / 2006$ \\
\hline
\end{tabular}


Appendix 2. Information pertaining to the difference in water levels measured in 2002 and 2006 in the Mississippi River Valley alluvial aquifer in eastern Arkansas.

[Horizontal coordinate information is referenced to the North American Datum of 1983 (NAD83); *, control wells for duplicate measurements by USGS and NRCS]

\begin{tabular}{|c|c|c|c|c|c|c|c|}
\hline Station name & $\begin{array}{c}\text { Latitude } \\
\text { (degrees, } \\
\text { minutes, } \\
\text { seconds) }\end{array}$ & $\begin{array}{c}\text { Longitude } \\
\text { (degrees, } \\
\text { minutes, } \\
\text { seconds) }\end{array}$ & $\begin{array}{c}2002 \text { water- } \\
\text { level date }\end{array}$ & $\begin{array}{c}2002 \text { depth } \\
\text { to water } \\
\text { (feet below } \\
\text { land-surface } \\
\text { datum) }\end{array}$ & $\begin{array}{c}2006 \text { water- } \\
\text { level date }\end{array}$ & $\begin{array}{c}2006 \text { depth } \\
\text { to water } \\
\text { (feet below } \\
\text { land-surface } \\
\text { datum) }\end{array}$ & $\begin{array}{c}\text { Water-level } \\
\text { difference } \\
2002 \text { to } 2006 \\
\text { (feet) }\end{array}$ \\
\hline \multicolumn{8}{|c|}{ Arkansas County } \\
\hline 02S04W11DBB1 & 343233 & 912415 & $3 / 7 / 2002$ & 100.48 & $4 / 10 / 2006$ & 100.7 & -0.2 \\
\hline 02S05W15AAB1 & 343213 & 913127 & $4 / 2 / 2002$ & 110.72 & $4 / 10 / 2006$ & 105.1 & 5.6 \\
\hline 03S02W27ABB1 & 342448 & 911251 & $3 / 7 / 2002$ & 69.8 & $4 / 06 / 2006$ & 67.53 & 2.3 \\
\hline 03S03W05CCD1 & 342737 & 912132 & $4 / 2 / 2002$ & 96.54 & $4 / 10 / 2006$ & 98.18 & -1.6 \\
\hline 03S03W27BBC1 & 342455 & 911944 & $4 / 2 / 2002$ & 90 & $4 / 10 / 2006$ & 91.65 & -1.7 \\
\hline 03S04W02BBB1 & 342831 & 912454 & $4 / 2 / 2002$ & 91.3 & $4 / 10 / 2006$ & 92.23 & -0.9 \\
\hline 03S04W03DCA16 & 342753 & 912515 & $3 / 6 / 2002$ & 99.1 & $5 / 02 / 2006$ & 100.45 & -1.4 \\
\hline 03S04W03DCA6 & 342753 & 912517 & $4 / 2 / 2002$ & 105.66 & 4/10/2006 & 99.73 & 5.9 \\
\hline 03S06W35ADD1 & 342411 & 913652 & $3 / 7 / 2002$ & 53.29 & $4 / 07 / 2006$ & 52.89 & 0.4 \\
\hline 04S01W04ACD2 & 342233 & 910733 & $3 / 7 / 2002$ & 6.48 & $4 / 06 / 2006$ & 5.74 & 0.7 \\
\hline 04S01W31DCB1 & 341753 & 910949 & $3 / 6 / 2002$ & 54.4 & $4 / 06 / 2006$ & 52.65 & 1.8 \\
\hline 04S02W11AAA1 & 342209 & 911123 & $3 / 7 / 2002$ & 67.37 & $4 / 06 / 2006$ & 69.1 & -1.7 \\
\hline 04S02W29CCC1 & 341846 & 911539 & $3 / 6 / 2002$ & 83.66 & $4 / 06 / 2006$ & 86.52 & -2.9 \\
\hline 04S03W17ADD1 & 342102 & 912058 & $3 / 6 / 2002$ & 106.18 & $4 / 10 / 2006$ & 107.72 & -1.5 \\
\hline 04S03W32BCB1 & 341820 & 912202 & $3 / 6 / 2002$ & 106.18 & $4 / 10 / 2006$ & 116.45 & -10.3 \\
\hline 04S04W02ABB1 & 342313 & 912424 & $3 / 6 / 2002$ & 108.07 & $4 / 10 / 2006$ & 108.63 & -0.6 \\
\hline 04S04W35ABC1 & 341835 & 912437 & $4 / 22 / 2002$ & 107 & $4 / 12 / 2006$ & 106 & 1.0 \\
\hline 04S05W16CDC1 & 342045 & 913321 & $3 / 7 / 2002$ & 71.1 & $4 / 07 / 2006$ & 69.35 & 1.8 \\
\hline 04S05W24DAA1 & 342001 & 912930 & $3 / 7 / 2002$ & 90.43 & $4 / 10 / 2006$ & 90.01 & 0.4 \\
\hline 04S06W15DBB1 & 342122 & 913827 & $3 / 7 / 2002$ & 31.24 & $4 / 07 / 2006$ & 33.06 & -1.8 \\
\hline 05S01W16BAB1 & 341552 & 910729 & $3 / 6 / 2002$ & 48.84 & $4 / 06 / 2006$ & 51.05 & -2.2 \\
\hline 05S02W16ABD1 & 341552 & 911358 & $3 / 6 / 2002$ & 73.91 & $4 / 06 / 2006$ & 83.3 & -9.4 \\
\hline 05S04W07CCC1 & 341555 & 912932 & $3 / 7 / 2002$ & 76.51 & $4 / 06 / 2006$ & 74.27 & 2.2 \\
\hline 05S04W32BBA1 & 341316 & 912822 & $3 / 7 / 2002$ & 59.36 & $4 / 06 / 2006$ & 57.42 & 1.9 \\
\hline 05S06W02DDD1 & 341724 & 913651 & $3 / 7 / 2002$ & 20.12 & $4 / 07 / 2006$ & 20.64 & -0.5 \\
\hline 05S06W07DDC1 & 341642 & 914130 & $3 / 7 / 2002$ & 9.36 & $4 / 07 / 2006$ & 2.39 & 7.0 \\
\hline 06S02W23DCD1 & 340853 & 911206 & $3 / 6 / 2002$ & 73.54 & $4 / 06 / 2006$ & 69.74 & 3.8 \\
\hline 06S03W10BBA1 & 341136 & 911954 & $3 / 6 / 2002$ & 83.12 & $4 / 06 / 2006$ & 81.98 & 1.1 \\
\hline 06S03W27AAA1 & 340858 & 911913 & $3 / 6 / 2002$ & 67.69 & $4 / 06 / 2006$ & 66.68 & 1.0 \\
\hline 07S02W04BBB1 & 340707 & 911452 & $3 / 6 / 2002$ & 31.12 & $4 / 06 / 2006$ & 42.2 & -11.1 \\
\hline 07S02W17BBA1 & 340530 & 911539 & $3 / 6 / 2002$ & 55.04 & $4 / 06 / 2006$ & 54.51 & 0.5 \\
\hline 07S03W18CCD1 & 340435 & 912316 & $3 / 6 / 2002$ & 43.37 & $4 / 06 / 2006$ & 42.41 & 1.0 \\
\hline 07S03W32BBC1 & 340240 & 912216 & $3 / 6 / 2002$ & 25.96 & $4 / 06 / 2006$ & 24.72 & 1.2 \\
\hline 07S04W01DDD1 & 340625 & 912327 & $3 / 6 / 2002$ & 47.41 & $4 / 06 / 2006$ & 47.1 & 0.3 \\
\hline 08S02W08ACA1 & 340041 & 911506 & $3 / 6 / 2002$ & 43.02 & $4 / 06 / 2006$ & 45.13 & -2.1 \\
\hline
\end{tabular}


Appendix 2. Information pertaining to the difference in water levels measured in 2002 and 2006 in the Mississippi River Valley alluvial aquifer in eastern Arkansas. - Continued

[Horizontal coordinate information is referenced to the North American Datum of 1983 (NAD83); *, control wells for duplicate measurements by USGS and NRCS]

\begin{tabular}{|c|c|c|c|c|c|c|c|}
\hline Station name & $\begin{array}{c}\text { Latitude } \\
\text { (degrees, } \\
\text { minutes, } \\
\text { seconds) }\end{array}$ & $\begin{array}{c}\text { Longitude } \\
\text { (degrees, } \\
\text { minutes, } \\
\text { seconds) }\end{array}$ & $\begin{array}{l}2002 \text { water- } \\
\text { level date }\end{array}$ & $\begin{array}{l}2002 \text { depth } \\
\text { to water } \\
\text { (feet below } \\
\text { land-surface } \\
\text { datum) }\end{array}$ & $\begin{array}{l}2006 \text { water- } \\
\text { level date }\end{array}$ & $\begin{array}{c}2006 \text { depth } \\
\text { to water } \\
\text { (feet below } \\
\text { land-surface } \\
\text { datum) }\end{array}$ & $\begin{array}{c}\text { Water-level } \\
\text { difference } \\
2002 \text { to } 2006 \\
\text { (feet) }\end{array}$ \\
\hline \multicolumn{8}{|c|}{ Arkansas County-Continued } \\
\hline 08S03WT2299 & 340147 & 912203 & $3 / 6 / 2002$ & 25.47 & $4 / 06 / 2006$ & 21.47 & 4.0 \\
\hline \multicolumn{8}{|c|}{ Ashley County } \\
\hline 15S04W26DCC1 & 332232 & 912902 & $2 / 26 / 2002$ & 30.68 & $3 / 15 / 2006$ & 31.09 & -0.4 \\
\hline 16S06W08CAA1 & 331941 & 914438 & $2 / 26 / 2002$ & 75.53 & $3 / 15 / 2006$ & 78.28 & -2.8 \\
\hline 16S06W27BAB1 & 331729 & 914240 & $4 / 30 / 2002$ & 81.66 & $3 / 15 / 2006$ & 83.84 & -2.2 \\
\hline 17S04W03ABB1 & 331528 & 913010 & $4 / 30 / 2002$ & 27.02 & $3 / 15 / 2006$ & 30.22 & -3.2 \\
\hline 17S04W15DDC1 & 331252 & 912954 & $2 / 26 / 2002$ & 24.22 & $3 / 15 / 2006$ & 26.5 & -2.3 \\
\hline 17S04W21ABA1 & 331252 & 913108 & $4 / 30 / 2002$ & 19.21 & $3 / 15 / 2006$ & 23.56 & -4.3 \\
\hline 17S06W01ADD1 & 331518 & 913956 & $2 / 26 / 2002$ & 81.25 & $3 / 15 / 2006$ & 83.58 & -2.3 \\
\hline 17S06W35CAC1 & 331049 & 914136 & $4 / 30 / 2002$ & 77.03 & $3 / 15 / 2006$ & 72.62 & 4.4 \\
\hline 18S05W11CCD1 & 330841 & 913538 & $4 / 29 / 2002$ & 20 & $3 / 25 / 2006$ & 22.8 & -2.8 \\
\hline 18S05W22DDA1 & 330712 & 913555 & $4 / 29 / 2002$ & 16 & $3 / 25 / 2006$ & 21 & -5.0 \\
\hline 18S08W01AAB1 & 331015 & 915225 & $2 / 26 / 2002$ & 86.18 & $3 / 15 / 2006$ & 86.63 & -0.5 \\
\hline 18S08W28DDD2 & 330625 & 915528 & $2 / 13 / 2002$ & 85.63 & $5 / 09 / 2006$ & 84.96 & 0.7 \\
\hline 19S04W06BAB2 & 330504 & 913329 & $2 / 26 / 2002$ & 22.23 & $3 / 15 / 2006$ & 23.67 & -1.4 \\
\hline 19S05W08ACA1 & 330405 & 913815 & $4 / 29 / 2002$ & 13 & $3 / 25 / 2006$ & 17.3 & -4.3 \\
\hline 19S05W16ABB1 & 330323 & 913718 & $4 / 29 / 2002$ & 19 & $3 / 25 / 2006$ & 24 & -5.0 \\
\hline 19S05W22DCD1 & 330139 & 913615 & $4 / 29 / 2002$ & 18 & $3 / 25 / 2006$ & 23.2 & -5.2 \\
\hline 19S06W07BCC1 & 330404 & 914608 & $2 / 26 / 2002$ & 31.43 & $3 / 15 / 2006$ & 31.04 & 0.4 \\
\hline \multicolumn{8}{|c|}{ Chicot County } \\
\hline 13S03W27AAA1 & 333253 & 912310 & $3 / 21 / 2002$ & 46 & $3 / 27 / 2006$ & 46 & 0.0 \\
\hline 13S03W34BAA1 & 333110 & 912539 & $2 / 25 / 2002$ & 37.82 & $3 / 16 / 2006$ & 40.32 & -2.5 \\
\hline 13S03W34CAA1 & 333136 & 912336 & $2 / 25 / 2002$ & 34.91 & $3 / 16 / 2006$ & 37.08 & -2.2 \\
\hline 13S03W35BAC1 & 333154 & 912246 & $2 / 25 / 2002$ & 35.71 & $3 / 16 / 2006$ & 39.58 & -3.9 \\
\hline 14S02W09BDD1 & 332859 & 911729 & $3 / 27 / 2002$ & 28 & $3 / 27 / 2006$ & 29 & -1.0 \\
\hline 14S02W18BBDD1 & 332859 & 912038 & $3 / 27 / 2002$ & 29 & $4 / 05 / 2006$ & 32 & -3.0 \\
\hline 14S03W07BBD1 & 333011 & 912620 & $2 / 25 / 2002$ & 24.19 & $3 / 16 / 2006$ & 26.56 & -2.4 \\
\hline 14S03W32CDB2 & 332613 & 912551 & $2 / 25 / 2002$ & 34.69 & $3 / 16 / 2006$ & 34.86 & -0.2 \\
\hline 15S02W20DDC $1 *$ & 332227 & 911920 & $3 / 21 / 2002$ & 29 & $3 / 22 / 2006$ & 29 & 0.0 \\
\hline 15S02W20DDC1* & 332227 & 911920 & $2 / 25 / 2002$ & 28.02 & $3 / 16 / 2006$ & 27.91 & 0.1 \\
\hline 16S03W11ADC1 & 331920 & 912234 & $2 / 26 / 2002$ & 27.34 & $3 / 16 / 2006$ & 28.93 & -1.6 \\
\hline 17S01E17CDA1 & 331259 & 910716 & $2 / 26 / 2002$ & 25.83 & $3 / 16 / 2006$ & 20.25 & 5.6 \\
\hline 17S01E18ADA1 & 331326 & 910758 & $2 / 26 / 2002$ & 17.03 & $3 / 16 / 2006$ & 11.04 & 6.0 \\
\hline 17S01W06BCC1 & 331501 & 911505 & $2 / 25 / 2002$ & 21.79 & $3 / 16 / 2006$ & 21.03 & 0.8 \\
\hline 17S02W10AAA1 & 331429 & 911712 & $4 / 30 / 2002$ & 26.2 & $3 / 16 / 2006$ & 26.35 & -0.2 \\
\hline
\end{tabular}


Appendix 2. Information pertaining to the difference in water levels measured in 2002 and 2006 in the Mississippi River Valley alluvial aquifer in eastern Arkansas.-Continued

[Horizontal coordinate information is referenced to the North American Datum of 1983 (NAD83); *, control wells for duplicate measurements by USGS and NRCS]

\begin{tabular}{|c|c|c|c|c|c|c|c|}
\hline Station name & $\begin{array}{l}\text { Latitude } \\
\text { (degrees, } \\
\text { minutes, } \\
\text { seconds) }\end{array}$ & $\begin{array}{c}\text { Longitude } \\
\text { (degrees, } \\
\text { minutes, } \\
\text { seconds) }\end{array}$ & $\begin{array}{l}2002 \text { water- } \\
\text { level date }\end{array}$ & $\begin{array}{c}2002 \text { depth } \\
\text { to water } \\
\text { (feet below } \\
\text { land-surface } \\
\text { datum) }\end{array}$ & $\begin{array}{l}2006 \text { water- } \\
\text { level date }\end{array}$ & $\begin{array}{l}2006 \text { depth } \\
\text { to water } \\
\text { (feet below } \\
\text { land-surface } \\
\text { datum) }\end{array}$ & $\begin{array}{c}\text { Water-level } \\
\text { difference } \\
2002 \text { to } 2006 \\
\text { (feet) }\end{array}$ \\
\hline \multicolumn{8}{|c|}{ Chicot County-Continued } \\
\hline 17S03W18CBC1 & 331257 & 912736 & $3 / 21 / 2002$ & 33 & $3 / 22 / 2006$ & 33 & 0.0 \\
\hline 17S03W28DBA1 & 331127 & 912441 & $2 / 25 / 2002$ & 23.26 & $3 / 16 / 2006$ & 24.33 & -1.1 \\
\hline 18S01W19DAB1 & 330709 & 911423 & $2 / 25 / 2002$ & 13.35 & $3 / 15 / 2006$ & 13.07 & 0.3 \\
\hline 18S01W33BAD1 & 330543 & 911245 & $3 / 28 / 2002$ & 12 & $3 / 22 / 2006$ & 18 & -6.0 \\
\hline 18S03W22ABA2 & 330728 & 912341 & $4 / 30 / 2002$ & 11.43 & $3 / 15 / 2006$ & 10.56 & 0.9 \\
\hline 19S01W17BCC1 & 330250 & 911406 & $2 / 25 / 2002$ & 18.62 & $3 / 15 / 2006$ & 19.76 & -1.1 \\
\hline 19S03W14ABB1 & 330304 & 912251 & $2 / 25 / 2002$ & 22.1 & $3 / 15 / 2006$ & 23.74 & -1.6 \\
\hline \multicolumn{8}{|c|}{ Clay County } \\
\hline 18N08E03DAB1 & 361323 & 901153 & $3 / 27 / 2002$ & 4.68 & $4 / 18 / 2006$ & 7.89 & -3.2 \\
\hline 18N08E11BAA1 & 361253 & 901117 & $4 / 15 / 2002$ & 7.7 & $4 / 12 / 2006$ & 7 & 0.7 \\
\hline 19N03E24AAA1 & 361655 & 904157 & $3 / 27 / 2002$ & 19.22 & $4 / 18 / 2006$ & 20.12 & -0.9 \\
\hline 19N04E11DAA1 & 361805 & 903621 & $4 / 15 / 2002$ & 22.6 & $4 / 12 / 2006$ & 23 & -0.4 \\
\hline 19N04E19AAA1 & 361654 & 904050 & $3 / 27 / 2002$ & 29.85 & $4 / 18 / 2006$ & 31.07 & -1.2 \\
\hline 19N04E19BAA1 & 361649 & 904125 & $4 / 15 / 2002$ & 21.9 & $4 / 12 / 2006$ & 22 & -0.1 \\
\hline 19N05E15BBD1 & 361716 & 903152 & $4 / 15 / 2002$ & 31.7 & $4 / 12 / 2006$ & 34 & -2.3 \\
\hline 19N06E18DBC1 & 361642 & 902815 & $4 / 15 / 2002$ & 34 & $4 / 13 / 2006$ & 37 & -3.0 \\
\hline 19N07E25BCB1 & 361519 & 901700 & $4 / 15 / 2002$ & 14.4 & $4 / 12 / 2006$ & 18 & -3.6 \\
\hline 19N08E08DCA1 & 361729 & 901402 & $4 / 15 / 2002$ & 26.3 & $4 / 12 / 2006$ & 7 & 19.3 \\
\hline 19N09E19CDC1 & 361539 & 900908 & $4 / 15 / 2002$ & 5.8 & $4 / 12 / 2006$ & 8 & -2.2 \\
\hline 20N03E25BAA1 & 362112 & 904225 & $4 / 15 / 2002$ & 23.3 & $4 / 13 / 2006$ & 22 & 1.3 \\
\hline 20N04E03ADA1 & 362425 & 903725 & $4 / 15 / 2002$ & 18.2 & $4 / 13 / 2006$ & 16 & 2.2 \\
\hline 20N04E06BB1 & 362444 & 904131 & $3 / 27 / 2002$ & 19.75 & $4 / 18 / 2006$ & 19.97 & -0.2 \\
\hline 20N05E22CAD1 & 362118 & 903132 & $4 / 15 / 2002$ & 26.7 & $4 / 12 / 2006$ & 31 & -4.3 \\
\hline 20N05E30CAC1 & 362003 & 903454 & $4 / 15 / 2002$ & 16.1 & $4 / 12 / 2006$ & 18 & -1.9 \\
\hline 20N05E34DBA1 & 361939 & 903117 & $3 / 27 / 2002$ & 27.25 & $4 / 18 / 2006$ & 29.31 & -2.1 \\
\hline 20N06E09BBA1 & 362327 & 902620 & $4 / 15 / 2002$ & 19.8 & $4 / 12 / 2006$ & 22 & -2.2 \\
\hline 20N06E28CCD1 & 362005 & 902630 & $4 / 15 / 2002$ & 26.5 & $4 / 12 / 2006$ & 29 & -2.5 \\
\hline 20N08E22BDC1 & 362111 & 901220 & $4 / 15 / 2002$ & 6.5 & $4 / 12 / 2006$ & 9 & -2.5 \\
\hline 20N09E09ABC1 & 362306 & 900642 & $4 / 15 / 2002$ & 4 & $4 / 12 / 2006$ & 8 & -4.0 \\
\hline 20N09E33DDC1 & 361904 & 900628 & $4 / 15 / 2002$ & 5.5 & $4 / 12 / 2006$ & 7 & -1.5 \\
\hline 21N03E15CBC1 & 362738 & 904453 & $4 / 15 / 2002$ & 8.9 & $4 / 13 / 2006$ & 11 & -2.1 \\
\hline 21N03E36CDD1 & 362450 & 904214 & $4 / 15 / 2002$ & 19.3 & $4 / 13 / 2006$ & 19 & 0.3 \\
\hline 21N04E09DBC1 & 362828 & 903853 & $4 / 15 / 2002$ & 10.1 & $4 / 13 / 2006$ & 13 & -2.9 \\
\hline 21N05E17ABB1 & 362755 & 903329 & $3 / 27 / 2002$ & 23.55 & 4/17/2006 & 23.23 & 0.3 \\
\hline 21N05E22BAB1 & 362704 & 903132 & $4 / 15 / 2002$ & 6.1 & $4 / 13 / 2006$ & 7 & -0.9 \\
\hline
\end{tabular}


Appendix 2. Information pertaining to the difference in water levels measured in 2002 and 2006 in the Mississippi River Valley alluvial aquifer in eastern Arkansas. - Continued

[Horizontal coordinate information is referenced to the North American Datum of 1983 (NAD83); *, control wells for duplicate measurements by USGS and NRCS]

\begin{tabular}{|c|c|c|c|c|c|c|c|}
\hline Station name & $\begin{array}{c}\text { Latitude } \\
\text { (degrees, } \\
\text { minutes, } \\
\text { seconds) }\end{array}$ & $\begin{array}{c}\text { Longitude } \\
\text { (degrees, } \\
\text { minutes, } \\
\text { seconds) }\end{array}$ & $\begin{array}{c}2002 \text { water- } \\
\text { level date }\end{array}$ & $\begin{array}{c}2002 \text { depth } \\
\text { to water } \\
\text { (feet below } \\
\text { land-surface } \\
\text { datum) }\end{array}$ & $\begin{array}{l}2006 \text { water- } \\
\text { level date }\end{array}$ & $\begin{array}{c}2006 \text { depth } \\
\text { to water } \\
\text { (feet below } \\
\text { land-surface } \\
\text { datum) }\end{array}$ & $\begin{array}{c}\text { Water-level } \\
\text { difference } \\
2002 \text { to } 2006 \\
\text { (feet) }\end{array}$ \\
\hline \multicolumn{8}{|c|}{ Clay County-Continued } \\
\hline 21N06E11BBB1 & 362839 & 902421 & $4 / 15 / 2002$ & 14.5 & $4 / 13 / 2006$ & 15 & -0.5 \\
\hline 21N06E28BB1 & 362605 & 902608 & $3 / 27 / 2002$ & 19.05 & $4 / 17 / 2006$ & 19.19 & -0.1 \\
\hline 21N07E01DDC1 & 362835 & 901607 & $4 / 15 / 2002$ & 21.5 & $4 / 13 / 2006$ & 26 & -4.5 \\
\hline 21N08E18CCC1 & 362651 & 901550 & $3 / 27 / 2002$ & 37.45 & $4 / 17 / 2006$ & 38.64 & -1.2 \\
\hline 21N08E36ABB1 & 362502 & 900958 & $5 / 2 / 2002$ & 1 & $4 / 18 / 2006$ & 4.05 & -3.1 \\
\hline 21N09E31BDA1 & 362447 & 900851 & $4 / 15 / 2002$ & 2 & $4 / 12 / 2006$ & 7 & -5.0 \\
\hline \multicolumn{8}{|c|}{ Craighead County } \\
\hline 13N01E03AAA1 & 354739 & 905753 & $4 / 3 / 2002$ & 55.2 & $3 / 01 / 2006$ & 54.7 & 0.5 \\
\hline 13N01E21CAB & 354434 & 905945 & $4 / 3 / 2002$ & 60 & $3 / 01 / 2006$ & 62 & -2.0 \\
\hline 13N01E23CAB1 & 354430 & 905736 & $4 / 3 / 2002$ & 66 & $3 / 01 / 2006$ & 68.5 & -2.5 \\
\hline 13N01E23DAA1 & 354435 & 905652 & $3 / 26 / 2002$ & 68.84 & $4 / 19 / 2006$ & 71.02 & -2.2 \\
\hline 13N02E02AAB1 & 354731 & 905032 & $4 / 3 / 2002$ & 82.4 & $3 / 01 / 2006$ & 92.2 & -9.8 \\
\hline 13N02E03AAA1 & 354733 & 905129 & $4 / 3 / 2002$ & 84.4 & $3 / 01 / 2006$ & 86.9 & -2.5 \\
\hline 13N03E23CDA1 & 354419 & 904434 & $4 / 3 / 2002$ & 78.3 & $3 / 02 / 2006$ & 79.6 & -1.3 \\
\hline 13N03E28CDB1 & 354322 & 904652 & $4 / 3 / 2002$ & 102.5 & $3 / 02 / 2006$ & 109 & -6.5 \\
\hline 13N03E29AAA1 & 354403 & 904713 & $3 / 26 / 2002$ & 101.45 & $4 / 19 / 2006$ & 103.76 & -2.3 \\
\hline 13N03E35AAA1 & 354308 & 904401 & $4 / 3 / 2002$ & 89 & $3 / 02 / 2006$ & 94 & -5.0 \\
\hline 13N04E12ABB1 & 354635 & 903656 & $3 / 26 / 2002$ & 25.48 & $4 / 19 / 2006$ & 23.82 & 1.7 \\
\hline 13N04E15DBA1 & 354521 & 903857 & $3 / 28 / 2002$ & 27.7 & $3 / 02 / 2006$ & 26.6 & 1.1 \\
\hline 13N04E26BCC1 & 354340 & 903829 & $3 / 28 / 2002$ & 29 & $3 / 02 / 2006$ & 26.5 & 2.5 \\
\hline 13N05E02CCC1 & 354648 & 903202 & $3 / 25 / 2002$ & 13 & $3 / 02 / 2006$ & 12.9 & 0.1 \\
\hline 13N05E06DCC1 & 354637 & 903547 & $3 / 25 / 2002$ & 20.9 & $3 / 02 / 2006$ & 20 & 0.9 \\
\hline 13N05E22BAD1 & 354449 & 903243 & $3 / 26 / 2002$ & 13.58 & $4 / 19 / 2006$ & 15.39 & -1.8 \\
\hline 13N05E24BAC1 & 354451 & 903045 & $3 / 25 / 2002$ & 7.8 & $3 / 02 / 2006$ & 12.2 & -4.4 \\
\hline 13N07E02CAB1 & 354642 & 901901 & $3 / 28 / 2002$ & 4 & $3 / 02 / 2006$ & 5 & -1.0 \\
\hline 13N07E05ABB1 & 354716 & 902158 & $3 / 28 / 2002$ & 6.5 & $3 / 02 / 2006$ & 5.5 & 1.0 \\
\hline 13N07E20BBA1 & 354440 & 902216 & $3 / 26 / 2002$ & 1.95 & $4 / 19 / 2006$ & 5.42 & -3.5 \\
\hline 13N07E35BCD1 & 354233 & 901837 & $3 / 28 / 2002$ & 7.8 & $3 / 02 / 2006$ & 12.3 & -4.5 \\
\hline 14N01E03ACB1 & 355246 & 905816 & $4 / 1 / 2002$ & 49.8 & $3 / 01 / 2006$ & 50.9 & -1.1 \\
\hline 14N01E10BAB1 & 355204 & 905828 & $4 / 1 / 2002$ & 48 & $3 / 01 / 2006$ & 51.1 & -3.1 \\
\hline 14N01E31DCA1 & 354817 & 910121 & 4/1/2002 & 56.5 & $3 / 01 / 2006$ & 59.1 & -2.6 \\
\hline 14N02E18BDD1 & 355041 & 905419 & $3 / 26 / 2002$ & 53.98 & $4 / 19 / 2006$ & 51.76 & 2.2 \\
\hline 14N02E22AAA1 & 355007 & 905129 & $4 / 1 / 2002$ & 71.4 & $3 / 01 / 2006$ & 74.8 & -3.4 \\
\hline 14N05E25ABB1 & 354921 & 903025 & $3 / 26 / 2002$ & 19.53 & $4 / 19 / 2006$ & 21.54 & -2.0 \\
\hline 14N06E06BAA1 & 355234 & 902934 & $3 / 25 / 2002$ & 22.5 & $3 / 02 / 2006$ & 22.4 & 0.1 \\
\hline
\end{tabular}


Appendix 2. Information pertaining to the difference in water levels measured in 2002 and 2006 in the Mississippi River Valley alluvial aquifer in eastern Arkansas.-Continued

[Horizontal coordinate information is referenced to the North American Datum of 1983 (NAD83); *, control wells for duplicate measurements by USGS and NRCS]

\begin{tabular}{|c|c|c|c|c|c|c|c|}
\hline Station name & $\begin{array}{c}\text { Latitude } \\
\text { (degrees, } \\
\text { minutes, } \\
\text { seconds) }\end{array}$ & $\begin{array}{c}\text { Longitude } \\
\text { (degrees, } \\
\text { minutes, } \\
\text { seconds) }\end{array}$ & $\begin{array}{c}2002 \text { water- } \\
\text { level date }\end{array}$ & $\begin{array}{c}2002 \text { depth } \\
\text { to water } \\
\text { (feet below } \\
\text { land-surface } \\
\text { datum) }\end{array}$ & $\begin{array}{c}2006 \text { water- } \\
\text { level date }\end{array}$ & $\begin{array}{c}2006 \text { depth } \\
\text { to water } \\
\text { (feet below } \\
\text { land-surface } \\
\text { datum) }\end{array}$ & $\begin{array}{c}\text { Water-level } \\
\text { difference } \\
2002 \text { to } 2006 \\
\text { (feet) }\end{array}$ \\
\hline \multicolumn{8}{|c|}{ Craighead -Continued } \\
\hline 14N06E27AAB1 & 354911 & 902559 & $3 / 26 / 2002$ & 0.65 & $4 / 19 / 2006$ & 3.1 & -2.5 \\
\hline 14N07E14DDC1 & 354956 & 901831 & $3 / 28 / 2002$ & 5.5 & $3 / 02 / 2006$ & 13.5 & -8.0 \\
\hline 14N07E26DBB1 & 354834 & 901843 & $3 / 26 / 2002$ & 3.1 & $4 / 19 / 2006$ & 9.76 & -6.7 \\
\hline 15N02E12DCB1 & 355626 & 904930 & $4 / 3 / 2002$ & 30.2 & $3 / 01 / 2006$ & 34.5 & -4.3 \\
\hline 15N03E19ADA1 & 355502 & 904802 & $3 / 26 / 2002$ & 43.43 & $4 / 19 / 2006$ & 49.16 & -5.7 \\
\hline 15N05E22BAB1 & 355513 & 903241 & $3 / 25 / 2002$ & 35.8 & $3 / 02 / 2006$ & 34.8 & 1.0 \\
\hline 15N06E04BAD1 & 355744 & 902706 & $3 / 25 / 2002$ & 14.6 & $3 / 02 / 2006$ & 15.5 & -0.9 \\
\hline 15N06E20DDD1 & 355426 & 902739 & $3 / 26 / 2002$ & 9.28 & 4/19/2006 & 11.22 & -1.9 \\
\hline 15N07E10DAB1 & 355622 & 901934 & $3 / 28 / 2002$ & 6 & $3 / 02 / 2006$ & 9.8 & -3.8 \\
\hline 15N07E10DBA1 & 355628 & 901944 & $3 / 26 / 2002$ & 6 & $4 / 19 / 2006$ & 9.44 & -3.4 \\
\hline 15N07E35DCB1 & 355241 & 901831 & $3 / 28 / 2002$ & 8 & $3 / 02 / 2006$ & 14.6 & -6.6 \\
\hline \multicolumn{8}{|c|}{ Crittenden County } \\
\hline 04N07E21AAD1 & 345644 & 902121 & $3 / 26 / 2002$ & 10.06 & $3 / 30 / 2006$ & 13.49 & -3.4 \\
\hline 05N07E08BDC1 & 350407 & 902234 & $4 / 18 / 2002$ & 21.7 & $4 / 27 / 2006$ & 22.8 & -1.1 \\
\hline 05N07E28CBA1 & 350121 & 902140 & $3 / 26 / 2002$ & 18.32 & $3 / 30 / 2006$ & 19.43 & -1.1 \\
\hline 05N07E34BAB1 & 350059 & 902030 & $3 / 26 / 2002$ & 15.09 & $3 / 30 / 2006$ & 19.46 & -4.4 \\
\hline 05N07E34CDD1 & 350010 & 902028 & $4 / 17 / 2002$ & 9.6 & $4 / 26 / 2006$ & 19 & -9.4 \\
\hline 05N08E11CCD2 & 350345 & 901308 & $3 / 21 / 2002$ & 27 & $3 / 30 / 2006$ & 28.32 & -1.3 \\
\hline 06N07E13BAA1 & 350850 & 901808 & $3 / 26 / 2002$ & 20.15 & $3 / 30 / 2006$ & 20.28 & -0.1 \\
\hline 06N07E14ABA1 & 350848 & 901858 & $4 / 12 / 2002$ & 20.8 & $4 / 26 / 2006$ & 20.4 & 0.4 \\
\hline 07N06E29CBC1 & 351152 & 902914 & $4 / 18 / 2002$ & 38.6 & $4 / 26 / 2006$ & 39.1 & -0.5 \\
\hline 07N07E31CCC1 & 351042 & 902359 & $3 / 26 / 2002$ & 32.46 & $3 / 30 / 2006$ & 34.92 & -2.5 \\
\hline 07N08E04BBD1 & 351538 & 901505 & $4 / 18 / 2002$ & 19.5 & $4 / 25 / 2006$ & 19.1 & 0.4 \\
\hline 07N09E05CDD1 & 351453 & 900934 & $3 / 26 / 2002$ & 14.47 & $3 / 30 / 2006$ & 16.94 & -2.5 \\
\hline 08N06E01DCC1 & 352021 & 902408 & $4 / 18 / 2002$ & 33 & $4 / 25 / 2006$ & 32 & 1.0 \\
\hline 08N06E06DDB1 & 352030 & 902920 & $4 / 19 / 2002$ & 32.3 & $4 / 25 / 2006$ & 30.2 & 2.1 \\
\hline 08N07E13CCC2 & 351828 & 901812 & $3 / 26 / 2002$ & 29.47 & $3 / 30 / 2006$ & 29.2 & 0.3 \\
\hline 08N07E14DAA2 & 351854 & 901833 & $3 / 26 / 2002$ & 30.08 & $3 / 30 / 2006$ & 29.98 & 0.1 \\
\hline 08N07E32DAA1 & 351618 & 902146 & $4 / 18 / 2002$ & 21.9 & $4 / 25 / 2006$ & 29 & -7.1 \\
\hline 08N08E06ABB1 & 352103 & 901644 & $4 / 19 / 2002$ & 28.8 & $4 / 25 / 2006$ & 29 & -0.2 \\
\hline 09N07E02CDB1 & 352537 & 901905 & $4 / 17 / 2002$ & 34.6 & $4 / 25 / 2006$ & 31.7 & 2.9 \\
\hline 09N07E10DDA1 & 352448 & 901925 & $3 / 26 / 2002$ & 28.15 & 3/30/2006 & 27.85 & 0.3 \\
\hline 09N07E31BAB1* & 352160 & 902327 & $4 / 18 / 2002$ & 33.6 & $4 / 25 / 2006$ & 31.8 & 1.8 \\
\hline 09N07E31BAB $1 *$ & 352160 & 902327 & $3 / 26 / 2002$ & 32.32 & $3 / 30 / 2006$ & 32.4 & -0.1 \\
\hline 09N08E04CDC1 & 352527 & 901444 & $4 / 19 / 2002$ & 28 & $4 / 25 / 2006$ & 24.1 & 3.9 \\
\hline
\end{tabular}


Appendix 2. Information pertaining to the difference in water levels measured in 2002 and 2006 in the Mississippi River Valley alluvial aquifer in eastern Arkansas. - Continued

[Horizontal coordinate information is referenced to the North American Datum of 1983 (NAD83); *, control wells for duplicate measurements by USGS and NRCS]

\begin{tabular}{|c|c|c|c|c|c|c|c|}
\hline Station name & $\begin{array}{c}\text { Latitude } \\
\text { (degrees, } \\
\text { minutes, } \\
\text { seconds) }\end{array}$ & $\begin{array}{l}\text { Longitude } \\
\text { (degrees, } \\
\text { minutes, } \\
\text { seconds) }\end{array}$ & $\begin{array}{l}2002 \text { water- } \\
\text { level date }\end{array}$ & $\begin{array}{c}2002 \text { depth } \\
\text { to water } \\
\text { (feet below } \\
\text { land-surface } \\
\text { datum) }\end{array}$ & $\begin{array}{l}2006 \text { water- } \\
\text { level date }\end{array}$ & $\begin{array}{c}2006 \text { depth } \\
\text { to water } \\
\text { (feet below } \\
\text { land-surface } \\
\text { datum) }\end{array}$ & $\begin{array}{c}\text { Water-level } \\
\text { difference } \\
2002 \text { to } 2006 \\
\text { (feet) }\end{array}$ \\
\hline \multicolumn{8}{|c|}{ Cross County } \\
\hline 06N02E11BDB1 & 350934 & 905132 & $4 / 18 / 2002$ & 61 & $4 / 26 / 2006$ & 62 & -1.0 \\
\hline 06N02E12AAA1 & 350934 & 904952 & $4 / 18 / 2002$ & 80 & $4 / 26 / 2006$ & 78 & 2.0 \\
\hline 07N01E05CDA1 & 351518 & 910049 & $3 / 21 / 2002$ & 70.18 & $3 / 29 / 2006$ & 73.64 & -3.5 \\
\hline 07N01E05DCA1 & 351514 & 910033 & $4 / 18 / 2002$ & 72 & $4 / 26 / 2006$ & 73 & -1.0 \\
\hline 07N01E06CAA1 & 351530 & 910154 & $4 / 18 / 2002$ & 69 & $4 / 24 / 2006$ & 73 & -4.0 \\
\hline 07N01E11AAA1 & 351501 & 905705 & $3 / 21 / 2002$ & 73.35 & $3 / 29 / 2006$ & 76.58 & -3.2 \\
\hline 07N01E33BBA1 & 351134 & 910010 & $4 / 18 / 2002$ & 70 & $4 / 24 / 2006$ & 68 & 2.0 \\
\hline 07N02E29DDC1 & 351138 & 905409 & $3 / 21 / 2002$ & 68.42 & $3 / 29 / 2006$ & 72.26 & -3.8 \\
\hline 07N03E05ADA1 & 351549 & 904739 & $3 / 21 / 2002$ & 110.71 & $3 / 29 / 2006$ & 124.05 & -13.3 \\
\hline 07N03E32DCC1 & 351045 & 904810 & $3 / 2 / 2002$ & 95.95 & $3 / 29 / 2006$ & 96.38 & -0.4 \\
\hline 07N04E04DBB1 & 351534 & 904021 & $4 / 18 / 2002$ & 30 & $5 / 01 / 2006$ & 30 & 0.0 \\
\hline 07N05E19CCC1 & 351238 & 903645 & $3 / 21 / 2002$ & 36.48 & $3 / 29 / 2006$ & 37.27 & -0.8 \\
\hline 07N05E24CCC1 & 351232 & 903121 & $4 / 18 / 2002$ & 36.6 & $4 / 26 / 2006$ & 34.9 & 1.7 \\
\hline 07N05E25ABA1 & 351229 & 903045 & $3 / 21 / 2002$ & 35.16 & $3 / 29 / 2006$ & 36.71 & -1.6 \\
\hline 08N01E16DBB1 & 351855 & 905933 & $4 / 18 / 2002$ & 84 & $4 / 27 / 2006$ & 84 & 0.0 \\
\hline 08N02E12DCC1 & 351938 & 905002 & $4 / 18 / 2002$ & 89 & $4 / 27 / 2006$ & 88 & 1.0 \\
\hline 08N02E17AAA1 & 351923 & 905354 & $4 / 18 / 2002$ & 83 & $4 / 27 / 2006$ & 85 & -2.0 \\
\hline 08N04E34CCD1 & 351605 & 903945 & $4 / 18 / 2002$ & 31 & $5 / 01 / 2006$ & 28 & 3.0 \\
\hline 08N05E32ADD1 & 351632 & 903440 & $3 / 21 / 2002$ & 31.98 & $3 / 29 / 2006$ & 29.57 & 2.4 \\
\hline 09N01E04ACD1 & 352608 & 905914 & $4 / 18 / 2002$ & 85 & $4 / 24 / 2006$ & 88 & -3.0 \\
\hline 09N01E33BBA2 & 352203 & 910001 & $3 / 21 / 2002$ & 77 & $3 / 29 / 2006$ & 81.1 & -4.1 \\
\hline 09N01E36AAB1 & 352155 & 905605 & $4 / 18 / 2002$ & 83 & $4 / 27 / 2006$ & 85 & -2.0 \\
\hline 09N02E20AAA1 & 352402 & 905342 & $4 / 18 / 2002$ & 91 & $4 / 27 / 2006$ & 94 & -3.0 \\
\hline 09N02E30CBB1 & 352243 & 905551 & $4 / 18 / 2002$ & 86 & $4 / 27 / 2006$ & 87 & -1.0 \\
\hline 09N03E17CDD1 & 352422 & 904753 & $4 / 18 / 2002$ & 102 & $4 / 27 / 2006$ & 99 & 3.0 \\
\hline 09N03E17DDC1 & 352409 & 904726 & $3 / 21 / 2002$ & 103.32 & $3 / 29 / 2006$ & 105.65 & -2.3 \\
\hline 09N05E32BCB1 & 352151 & 903525 & $4 / 18 / 2002$ & 37 & $5 / 04 / 2006$ & 30 & 7.0 \\
\hline 09N05E32BDB1 & 352151 & 903512 & $3 / 21 / 2002$ & 31.95 & $3 / 29 / 2006$ & 30.63 & 1.3 \\
\hline \multicolumn{8}{|c|}{ Desha County } \\
\hline 07S01E19ABA1 & 340428 & 910303 & $4 / 18 / 2002$ & 13 & $4 / 12 / 2006$ & 21 & -8.0 \\
\hline 08S03W33ABD1 & 335803 & 912338 & $3 / 4 / 2002$ & 6.14 & $3 / 17 / 2006$ & 7.24 & -1.1 \\
\hline 09S01W08BDA1 & 335608 & 911234 & $4 / 11 / 2002$ & 23 & $4 / 07 / 2006$ & 28 & -5.0 \\
\hline 09S01W15CBB1 & 335501 & 911055 & $4 / 11 / 2002$ & 37 & $4 / 07 / 2006$ & 36 & 1.0 \\
\hline 09S02W26DDC1 & 335257 & 911530 & $2 / 27 / 2002$ & 30.35 & $5 / 09 / 2006$ & 29.33 & 1.0 \\
\hline 09S03W05BAC1 & 335704 & 912506 & $4 / 11 / 2002$ & 43 & $4 / 07 / 2006$ & 42 & 1.0 \\
\hline
\end{tabular}


Appendix 2. Information pertaining to the difference in water levels measured in 2002 and 2006 in the Mississippi River Valley alluvial aquifer in eastern Arkansas.-Continued

[Horizontal coordinate information is referenced to the North American Datum of 1983 (NAD83); *, control wells for duplicate measurements by USGS and NRCS]

\begin{tabular}{|c|c|c|c|c|c|c|c|}
\hline Station name & $\begin{array}{c}\text { Latitude } \\
\text { (degrees, } \\
\text { minutes, } \\
\text { seconds) }\end{array}$ & $\begin{array}{c}\text { Longitude } \\
\text { (degrees, } \\
\text { minutes, } \\
\text { seconds) }\end{array}$ & $\begin{array}{l}2002 \text { water- } \\
\text { level date }\end{array}$ & $\begin{array}{c}2002 \text { depth } \\
\text { to water } \\
\text { (feet below } \\
\text { land-surface } \\
\text { datum) }\end{array}$ & $\begin{array}{c}2006 \text { water- } \\
\text { level date }\end{array}$ & $\begin{array}{c}2006 \text { depth } \\
\text { to water } \\
\text { (feet below } \\
\text { land-surface } \\
\text { datum) }\end{array}$ & $\begin{array}{c}\text { Water-level } \\
\text { difference } \\
2002 \text { to } 2006 \\
\text { (feet) }\end{array}$ \\
\hline \multicolumn{8}{|c|}{ Desha County -Continued } \\
\hline 09S03W13BAB1 & 335500 & 911922 & $4 / 11 / 2002$ & 31 & $4 / 07 / 2006$ & 32 & -1.0 \\
\hline 09S03W17DCB1 & 335448 & 912457 & $3 / 4 / 2002$ & 33.74 & $3 / 17 / 2006$ & 32.95 & 0.8 \\
\hline 09S04W06BCA1 & 335756 & 913243 & $3 / 4 / 2002$ & 32.78 & $3 / 17 / 2006$ & 34.92 & -2.1 \\
\hline 10S01W23CDA1 & 335305 & 911032 & $4 / 11 / 2002$ & 27 & $4 / 07 / 2006$ & 26 & 1.0 \\
\hline 10S02W11ADD1 & 335045 & 911517 & $4 / 11 / 2002$ & 27 & $4 / 07 / 2006$ & 28 & -1.0 \\
\hline 10S02W24DBC1 & 334850 & 911453 & $2 / 27 / 2002$ & 25.62 & $3 / 17 / 2006$ & 25.53 & 0.1 \\
\hline 10S03W26CAA1 & 334806 & 912145 & $2 / 27 / 2002$ & 42.94 & $3 / 17 / 2006$ & 44.8 & -1.9 \\
\hline 11S02W15ADD1 & 334446 & 911635 & $4 / 11 / 2002$ & 32 & $4 / 07 / 2006$ & 34 & -2.0 \\
\hline 11S03W16CBA1 & 334439 & 912433 & $4 / 11 / 2002$ & 32 & $4 / 07 / 2006$ & 31 & 1.0 \\
\hline 11S03W31BBA1 & 334228 & 912651 & $2 / 27 / 2002$ & 31.47 & $3 / 17 / 2006$ & 35.14 & -3.7 \\
\hline 12S01W33BAA1 & 333718 & 911205 & $2 / 27 / 2002$ & 25.7 & $3 / 17 / 2006$ & 23.92 & 1.8 \\
\hline 13S02W17ADA1 & 333421 & 911858 & $4 / 11 / 2002$ & 43 & $4 / 07 / 2006$ & 45 & -2.0 \\
\hline 13S02W27CAC1 & 333224 & 911735 & $2 / 27 / 2002$ & 30.38 & $3 / 17 / 2006$ & 30.83 & -0.5 \\
\hline 13S02W32DBD1 & 333126 & 911917 & $4 / 11 / 2002$ & 38 & $4 / 07 / 2006$ & 43 & -5.0 \\
\hline 13S03W10DAA1 & 333506 & 912302 & $2 / 27 / 2002$ & 44.2 & $3 / 17 / 2006$ & 47.12 & -2.9 \\
\hline 13S03W11CAB1 & 333503 & 912241 & $4 / 11 / 2002$ & 46 & $4 / 07 / 2006$ & 51 & -5.0 \\
\hline \multicolumn{8}{|c|}{ Drew County } \\
\hline 11S04W08DBA1 & 334532 & 913136 & $2 / 27 / 2002$ & 23.98 & $3 / 16 / 2006$ & 25.14 & -1.2 \\
\hline 11S05W08CCC1 & 334546 & 913837 & $2 / 27 / 2002$ & 35.05 & $3 / 16 / 2006$ & 36.48 & -1.4 \\
\hline 12S04W03ABB1 & 334134 & 912946 & $2 / 27 / 2002$ & 22.92 & $3 / 16 / 2006$ & 24.23 & -1.3 \\
\hline 12S04W25DBB1 & 333739 & 912738 & 4/9/2002 & 28 & $4 / 13 / 2006$ & 34 & -6.0 \\
\hline 13S04W09ACD1 & 333512 & 913034 & 4/9/2002 & 16.4 & $4 / 13 / 2006$ & 19 & -2.6 \\
\hline 13S04W28CDD1 & 333206 & 913100 & $2 / 26 / 2002$ & 18.09 & $3 / 16 / 2006$ & 17.63 & 0.5 \\
\hline 13S04W33BAA1 & 333206 & 913100 & $2 / 26 / 2002$ & 18.09 & $3 / 16 / 2006$ & 18.14 & -0.1 \\
\hline 13S05W29ADA1 & 333248 & 913747 & $5 / 1 / 2002$ & 46.04 & $3 / 16 / 2006$ & 40.5 & 5.5 \\
\hline 13S06W03DDC1 & 333545 & 914202 & $2 / 26 / 2002$ & 59.16 & $3 / 16 / 2006$ & 62.17 & -3.0 \\
\hline 13S06W21DAA1 & 333324 & 914258 & $4 / 1 / 2002$ & 81 & $4 / 13 / 2006$ & 74 & 7.0 \\
\hline 14S04W03ADD1 & 333050 & 912929 & 4/9/2002 & 24 & $4 / 13 / 2006$ & 27 & -3.0 \\
\hline 14S04W05CBA1 & 333047 & 913218 & $4 / 10 / 2002$ & 13 & $4 / 13 / 2006$ & 14 & -1.0 \\
\hline 14S04W05CBC1 & 333042 & 913226 & $4 / 10 / 2002$ & 14 & $4 / 13 / 2006$ & 15 & -1.0 \\
\hline 14S04W22CAA1 & 332805 & 912957 & 4/9/2002 & 16 & $4 / 13 / 2006$ & 13 & 3.0 \\
\hline 14S05W23DCB1 & 332802 & 913512 & $2 / 27 / 2002$ & 22.7 & $3 / 16 / 2006$ & 29.77 & -7.1 \\
\hline \multicolumn{8}{|c|}{ Greene County } \\
\hline 16N03E03BA1 & 360316 & 904516 & $3 / 28 / 2002$ & 28.81 & $4 / 18 / 2006$ & 30.73 & -1.9 \\
\hline 16N03E05BBB1 & 360316 & 904750 & $4 / 11 / 2002$ & 26.9 & $4 / 11 / 2006$ & 30.5 & -3.6 \\
\hline
\end{tabular}


Appendix 2. Information pertaining to the difference in water levels measured in 2002 and 2006 in the Mississippi River Valley alluvial aquifer in eastern Arkansas.-Continued

[Horizontal coordinate information is referenced to the North American Datum of 1983 (NAD83); *, control wells for duplicate measurements by USGS and NRCS]

\begin{tabular}{|c|c|c|c|c|c|c|c|}
\hline Station name & $\begin{array}{c}\text { Latitude } \\
\text { (degrees, } \\
\text { minutes, } \\
\text { seconds) }\end{array}$ & $\begin{array}{c}\text { Longitude } \\
\text { (degrees, } \\
\text { minutes, } \\
\text { seconds) }\end{array}$ & $\begin{array}{l}2002 \text { water- } \\
\text { level date }\end{array}$ & $\begin{array}{l}2002 \text { depth } \\
\text { to water } \\
\text { (feet below } \\
\text { land-surface } \\
\text { datum) }\end{array}$ & $\begin{array}{l}2006 \text { water- } \\
\text { level date }\end{array}$ & $\begin{array}{l}2006 \text { depth } \\
\text { to water } \\
\text { (feet below } \\
\text { land-surface } \\
\text { datum) }\end{array}$ & $\begin{array}{c}\text { Water-level } \\
\text { difference } \\
2002 \text { to } 2006 \\
\text { (feet) }\end{array}$ \\
\hline \multicolumn{8}{|c|}{ Greene County-Continued } \\
\hline 16N03E16DDD1 & 360049 & 904547 & $4 / 11 / 2002$ & 26 & $4 / 11 / 2006$ & 26.8 & -0.8 \\
\hline 16N03E29ACC1 & 355926 & 904722 & $4 / 11 / 2002$ & 29.8 & $4 / 11 / 2006$ & 31.5 & -1.7 \\
\hline 16N06E09ABB1 & 360215 & 902651 & $4 / 5 / 2002$ & 52.1 & $4 / 11 / 2006$ & 49.9 & 2.2 \\
\hline 16N06E21BAA1 & 360031 & 902705 & $4 / 5 / 2002$ & 29.8 & $4 / 11 / 2006$ & 27.1 & 2.7 \\
\hline 16N06E28ABB1 & 355938 & 902657 & $3 / 27 / 2002$ & 28.42 & $4 / 18 / 2006$ & 25.76 & 2.7 \\
\hline 17N03E02DCC1 & 360806 & 904352 & $4 / 11 / 2002$ & 29.6 & $4 / 11 / 2006$ & 20.2 & 9.4 \\
\hline 17N04E07AD1 & 360718 & 904122 & $4 / 11 / 2002$ & 38.8 & $4 / 11 / 2006$ & 42.6 & -3.8 \\
\hline 17N04E30CDC1 & 360409 & 904218 & $3 / 28 / 2002$ & 34.96 & $4 / 18 / 2006$ & 37.39 & -2.4 \\
\hline 17N06E15ABC1 & 360631 & 902546 & 4/5/2002 & 35.2 & $4 / 11 / 2006$ & 31.1 & 4.1 \\
\hline 17N07E03CCC1 & 360744 & 901951 & $4 / 5 / 2002$ & 4.1 & $4 / 11 / 2006$ & 5.8 & -1.7 \\
\hline 17N07E18ABB1 & 360638 & 902235 & $3 / 27 / 2002$ & 5.48 & $4 / 18 / 2006$ & 8.86 & -3.4 \\
\hline 17N07E29CBC1 & 360419 & 902201 & $4 / 5 / 2002$ & 1.7 & $4 / 11 / 2006$ & 6.3 & -4.6 \\
\hline 18N04E04AAC1 & 361356 & 903854 & $4 / 11 / 2002$ & 28.6 & $4 / 11 / 2006$ & 32.6 & -4.0 \\
\hline 18N04E21CBD1 & 361052 & 903725 & $3 / 27 / 2002$ & 53.04 & $4 / 18 / 2006$ & 55.63 & -2.6 \\
\hline 18N07E17BAB1 & 361203 & 902105 & $4 / 5 / 2002$ & 6.4 & $4 / 11 / 2006$ & 11.3 & -4.9 \\
\hline 18N07E20BBA1 & 361110 & 902113 & $3 / 27 / 2002$ & 3.77 & $4 / 18 / 2006$ & 10.92 & -7.2 \\
\hline 19N03E26AD1 & 361601 & 904258 & $3 / 27 / 2002$ & 27.7 & $4 / 18 / 2006$ & 29.5 & -1.8 \\
\hline 19N03E33DDD1 & 361418 & 904516 & $4 / 11 / 2002$ & 33.3 & $4 / 11 / 2006$ & 36 & -2.7 \\
\hline 19N05E34AAD1 & 361437 & 903102 & $4 / 11 / 2002$ & 36.8 & $4 / 11 / 2006$ & 34.2 & 2.6 \\
\hline \multicolumn{8}{|c|}{ Independence County } \\
\hline 11N04W02ABB1 & 353650 & 912416 & $4 / 10 / 2002$ & 9.1 & $4 / 04 / 2006$ & 10.2 & -1.1 \\
\hline 12N04W14DD1 & 353929 & 912236 & $3 / 27 / 2002$ & 15.74 & $3 / 23 / 2006$ & 27.03 & -11.3 \\
\hline 12N04W34CBB1 & 353720 & 912513 & $3 / 27 / 2002$ & 8.6 & $3 / 23 / 2006$ & 23.51 & -14.9 \\
\hline 12N05W36AAA1 & 353738 & 912827 & $3 / 27 / 2002$ & 12.62 & $3 / 23 / 2006$ & 25.64 & -13.0 \\
\hline 14N03W12CAB1 & 355152 & 911541 & $4 / 10 / 2002$ & 1 & $4 / 04 / 2006$ & 2.8 & -1.8 \\
\hline 14N03W14CBB1 & 355101 & 911703 & $4 / 10 / 2002$ & 13.5 & $4 / 04 / 2006$ & 2.4 & 11.1 \\
\hline 14N03W14DAA2 & 355107 & 911602 & $3 / 27 / 2002$ & 0.7 & $3 / 23 / 2006$ & 4.89 & -4.2 \\
\hline 14N03W14DBB1 & 355106 & 911640 & $3 / 27 / 2002$ & 1.5 & $3 / 23 / 2006$ & 5.93 & -4.4 \\
\hline \multicolumn{8}{|c|}{ Jackson County } \\
\hline 09N01W15DDD1 & 352357 & 910433 & $4 / 8 / 2002$ & 58 & $4 / 20 / 2006$ & 54.6 & 3.4 \\
\hline 09N01W22ADD1 & 352332 & 910433 & $3 / 21 / 2002$ & 59.07 & $3 / 27 / 2006$ & 61.72 & -2.6 \\
\hline 09N01W30BAC1 & 352258 & 910813 & $4 / 3 / 2002$ & 42.6 & $4 / 20 / 2006$ & 44.5 & -1.9 \\
\hline 09N02W32BBB1 & 352215 & 911344 & $4 / 3 / 2002$ & 31.5 & $4 / 20 / 2006$ & 34 & -2.5 \\
\hline 09N02W32CBB1 & 352152 & 911348 & $3 / 21 / 2002$ & 29.92 & $3 / 27 / 2006$ & 30.29 & -0.4 \\
\hline 10N01W05ADD1 & 353132 & 910702 & $3 / 28 / 2002$ & 44.8 & $4 / 20 / 2006$ & 47.3 & -2.5 \\
\hline
\end{tabular}


Appendix 2. Information pertaining to the difference in water levels measured in 2002 and 2006 in the Mississippi River Valley alluvial aquifer in eastern Arkansas.-Continued

[Horizontal coordinate information is referenced to the North American Datum of 1983 (NAD83); *, control wells for duplicate measurements by USGS and NRCS

\begin{tabular}{|c|c|c|c|c|c|c|c|}
\hline Station name & $\begin{array}{c}\text { Latitude } \\
\text { (degrees, } \\
\text { minutes, } \\
\text { seconds) }\end{array}$ & $\begin{array}{c}\text { Longitude } \\
\text { (degrees, } \\
\text { minutes, } \\
\text { seconds) }\end{array}$ & $\begin{array}{c}2002 \text { water- } \\
\text { level date }\end{array}$ & $\begin{array}{c}2002 \text { depth } \\
\text { to water } \\
\text { (feet below } \\
\text { land-surface } \\
\text { datum) }\end{array}$ & $\begin{array}{c}2006 \text { water- } \\
\text { level date }\end{array}$ & $\begin{array}{c}2006 \text { depth } \\
\text { to water } \\
\text { (feet below } \\
\text { land-surface } \\
\text { datum) }\end{array}$ & $\begin{array}{c}\text { Water-level } \\
\text { difference } \\
2002 \text { to } 2006 \\
\text { (feet) }\end{array}$ \\
\hline \multicolumn{8}{|c|}{ Jackson County-Continued } \\
\hline 10N01W10ABA1 & 353055 & 910445 & $3 / 28 / 2002$ & 55.7 & $4 / 20 / 2006$ & 59.2 & -3.5 \\
\hline 10N02W29ABB1 & 352829 & 911312 & $3 / 21 / 2002$ & 27.93 & $3 / 27 / 2006$ & 27.94 & -0.0 \\
\hline 11N01W26AAD1* & 353330 & 910323 & $3 / 28 / 2002$ & 63.7 & $4 / 20 / 2006$ & 66.2 & -2.5 \\
\hline 11N01W26AAD1* & 353330 & 910323 & $3 / 21 / 2002$ & 64.77 & $3 / 24 / 2006$ & 67.06 & -2.3 \\
\hline 11N01W29AAD1 & 353339 & 910635 & $3 / 27 / 2002$ & 39.52 & $3 / 24 / 2006$ & 39.07 & 0.5 \\
\hline 11N02W25BBD1 & 353322 & 910855 & $3 / 28 / 2002$ & 25.1 & $4 / 20 / 2006$ & 26 & -0.9 \\
\hline 11N03W06DAB1 & 353655 & 912009 & $3 / 21 / 2002$ & 10.15 & $3 / 24 / 2006$ & 22.23 & -12.1 \\
\hline 12N01W11BCB1 & 354127 & 910416 & $3 / 28 / 2002$ & 37.3 & $4 / 12 / 2006$ & 38.2 & -0.9 \\
\hline 12N01W30CCC2 & 353812 & 910821 & $3 / 28 / 2002$ & 32.9 & $4 / 12 / 2006$ & 33 & -0.1 \\
\hline 12N01W36CBC1 & 353724 & 910317 & $3 / 28 / 2002$ & 48.9 & $4 / 12 / 2006$ & 52.9 & -4.0 \\
\hline $12 \mathrm{~N} 02 \mathrm{~W} 25 \mathrm{ABB} 2$ & 353910 & 910852 & $3 / 21 / 2002$ & 36.86 & $3 / 24 / 2006$ & 33.24 & 3.6 \\
\hline 13N01W20AAA1 & 354514 & 910627 & $3 / 21 / 2002$ & 38.59 & $3 / 27 / 2006$ & 39.26 & -0.7 \\
\hline 13N03W15CDD1 & 354526 & 911749 & $3 / 21 / 2002$ & 14.77 & $3 / 27 / 2006$ & 16.93 & -2.2 \\
\hline 13N03W15DCB1 & 354540 & 911718 & $4 / 2 / 2002$ & 11.3 & $4 / 12 / 2006$ & 17.9 & -6.6 \\
\hline 13N03W36ABB1 & 354337 & 911532 & $4 / 3 / 2002$ & 16.2 & $4 / 12 / 2006$ & 16.6 & -0.4 \\
\hline 14N01W08AAA1 & 355216 & 910623 & $4 / 3 / 2002$ & 34.5 & $4 / 12 / 2006$ & 36 & -1.5 \\
\hline 14N01W09AAA1 & 355220 & 910515 & $3 / 27 / 2002$ & 40.36 & $3 / 27 / 2006$ & 42.43 & -2.1 \\
\hline 14N01W19BBB1 & 355032 & 910823 & $4 / 3 / 2002$ & 31.2 & $4 / 12 / 2006$ & 32 & -0.8 \\
\hline 14N01W26BCB1 & 354922 & 910407 & $4 / 3 / 2002$ & 43.1 & $4 / 12 / 2006$ & 43.3 & -0.2 \\
\hline 14N01W33CCD1 & 354759 & 910610 & $3 / 28 / 2002$ & 38.2 & $4 / 12 / 2006$ & 39.3 & -1.1 \\
\hline 14N02W22BBC1 & 355026 & 911145 & $4 / 3 / 2002$ & 24.5 & $4 / 12 / 2006$ & 28 & -3.5 \\
\hline \multicolumn{8}{|c|}{ Jefferson County } \\
\hline 03S08W24BBC1 & 342620 & 914953 & $3 / 5 / 2002$ & 48.62 & $4 / 03 / 2006$ & 51.03 & -2.4 \\
\hline 03S09W06DDA1 & 342840 & 920037 & $3 / 5 / 2002$ & 36.79 & $4 / 03 / 2006$ & 37.94 & -1.1 \\
\hline 03S09W22AAA1 & 342640 & 915728 & $5 / 2 / 2002$ & 38.5 & $4 / 06 / 2006$ & 40.7 & -2.2 \\
\hline 03S09W29CBD1 & 342517 & 920023 & $3 / 5 / 2002$ & 25.9 & $4 / 03 / 2006$ & 29.11 & -3.2 \\
\hline 03S09W36ACC1 & 342428 & 915555 & $5 / 2 / 2002$ & 38 & $4 / 07 / 2006$ & 27.9 & 10.1 \\
\hline 03S10W25BCA2 & 342537 & 920242 & $5 / 3 / 2002$ & 18 & $4 / 06 / 2006$ & 18.8 & -0.8 \\
\hline 03S10W26BBB2 & 342427 & 920250 & $5 / 3 / 2002$ & 17.5 & $4 / 06 / 2006$ & 15.7 & 1.8 \\
\hline 04S07W35DDB1 & 341836 & 914347 & $4 / 26 / 2002$ & 26.5 & $4 / 06 / 2006$ & 27.3 & -0.8 \\
\hline 04S08W13DCB1 & 342123 & 914926 & $3 / 5 / 2002$ & 43.7 & $4 / 03 / 2006$ & 48.34 & -4.6 \\
\hline 04S09W02CBD1 & 342325 & 915717 & $5 / 2 / 2002$ & 32 & $4 / 06 / 2006$ & 33.3 & -1.3 \\
\hline 04S09W32DDA1 & 341859 & 920009 & $5 / 3 / 2002$ & 18 & $4 / 06 / 2006$ & 23 & -5.0 \\
\hline 05S06W31CAA1 & 341330 & 914206 & $3 / 5 / 2002$ & 15.41 & $4 / 04 / 2006$ & 19.85 & -4.4 \\
\hline 05S07W29DDD1 & 341411 & 914654 & $4 / 26 / 2002$ & 13.5 & $4 / 06 / 2006$ & 14.2 & -0.7 \\
\hline
\end{tabular}


Appendix 2. Information pertaining to the difference in water levels measured in 2002 and 2006 in the Mississippi River Valley alluvial aquifer in eastern Arkansas.-Continued

[Horizontal coordinate information is referenced to the North American Datum of 1983 (NAD83); *, control wells for duplicate measurements by USGS and NRCS]

\begin{tabular}{|c|c|c|c|c|c|c|c|}
\hline Station name & $\begin{array}{c}\text { Latitude } \\
\text { (degrees, } \\
\text { minutes, } \\
\text { seconds) }\end{array}$ & $\begin{array}{l}\text { Longitude } \\
\text { (degrees, } \\
\text { minutes, } \\
\text { seconds) }\end{array}$ & $\begin{array}{l}2002 \text { water- } \\
\text { level date }\end{array}$ & $\begin{array}{c}2002 \text { depth } \\
\text { to water } \\
\text { (feet below } \\
\text { land-surface } \\
\text { datum) }\end{array}$ & $\begin{array}{l}2006 \text { water- } \\
\text { level date }\end{array}$ & $\begin{array}{l}2006 \text { depth } \\
\text { to water } \\
\text { (feet below } \\
\text { land-surface } \\
\text { datum) }\end{array}$ & $\begin{array}{c}\text { Water-level } \\
\text { difference } \\
2002 \text { to } 2006 \\
\text { (feet) }\end{array}$ \\
\hline \multicolumn{8}{|c|}{ Jefferson County-Continued } \\
\hline 05S08W12DAA1 & 341712 & 914907 & $3 / 5 / 2002$ & 16.71 & $4 / 03 / 2006$ & 18.98 & -2.3 \\
\hline 06S05W15BCA1 & 341023 & 913245 & $3 / 5 / 2002$ & 20.2 & $4 / 04 / 2006$ & 18.58 & 1.6 \\
\hline 06S06W23AAD1 & 341007 & 913712 & $3 / 5 / 2002$ & 19.26 & $4 / 04 / 2006$ & 21.23 & -2.0 \\
\hline 06S07W14BAA1 & 341125 & 914426 & $3 / 5 / 2002$ & 15.14 & $4 / 04 / 2006$ & 16.07 & -0.9 \\
\hline 07S07W16BAA1 & 340722 & 914828 & $4 / 26 / 2002$ & 29 & $4 / 06 / 2006$ & 28 & 1.0 \\
\hline 07S08W06BAA1 & 340859 & 915647 & $3 / 5 / 2002$ & 19.64 & $4 / 04 / 2006$ & 18.65 & 1.0 \\
\hline \multicolumn{8}{|c|}{ Lawrence County } \\
\hline 15N01E11ADD1 & 355657 & 905638 & $4 / 11 / 2002$ & 40.8 & $4 / 18 / 2006$ & 44.4 & -3.6 \\
\hline 15N01E26DDA1 & 355402 & 905639 & $3 / 27 / 2002$ & 49.55 & $4 / 17 / 2006$ & 51.93 & -2.4 \\
\hline 15N01W03BAB1 & 355831 & 910441 & $4 / 15 / 2002$ & 37.5 & $4 / 18 / 2006$ & 36.2 & 1.3 \\
\hline 15N01W35CBB1 & 355336 & 910356 & $3 / 27 / 2002$ & 43.05 & $4 / 17 / 2006$ & 44.98 & -1.9 \\
\hline 16N01E11DAC2 & 360203 & 905639 & $3 / 27 / 2002$ & 44.16 & $4 / 17 / 2006$ & 46.76 & -2.6 \\
\hline 16N01E35AAA1 & 355908 & 905632 & $4 / 10 / 2002$ & 41.8 & $4 / 18 / 2006$ & 49.2 & -7.4 \\
\hline 16N01W30DDC1 & 355937 & 910723 & $4 / 15 / 2002$ & 22 & $4 / 18 / 2006$ & 21.6 & 0.4 \\
\hline 16N02E09AAD1 & 360219 & 905212 & $4 / 12 / 2002$ & 38 & $4 / 18 / 2006$ & 40.3 & -2.3 \\
\hline 16N02E34CBB1 & 355831 & 905208 & $4 / 10 / 2002$ & 42 & $4 / 18 / 2006$ & 48.1 & -6.1 \\
\hline 17N01E02BBA1 & 360901 & 905707 & $4 / 15 / 2002$ & 11.4 & $4 / 18 / 2006$ & 15 & -3.6 \\
\hline 17N01W36AAB1 & 360435 & 910158 & 4/9/2002 & 12.3 & $4 / 18 / 2006$ & 13.1 & -0.8 \\
\hline 17N02E04DCA1 & 360758 & 905224 & $4 / 12 / 2002$ & 37.5 & $4 / 18 / 2006$ & 40.9 & -3.4 \\
\hline 17N02E19CDC1 & 360516 & 905449 & $3 / 27 / 2002$ & 38.44 & $4 / 17 / 2006$ & 38.94 & -0.5 \\
\hline 17N02E25CBD1 & 360423 & 904948 & $4 / 12 / 2002$ & 34.1 & $4 / 18 / 2006$ & 38.1 & -4.0 \\
\hline \multicolumn{8}{|c|}{ Lee County } \\
\hline 01N01E04AAB1 & 344358 & 910015 & $4 / 10 / 2002$ & 25 & $5 / 02 / 2006$ & 29.3 & -4.3 \\
\hline 01N01E09CCC1 & 344215 & 910054 & $4 / 12 / 2002$ & 28 & $5 / 02 / 2006$ & 32.5 & -4.5 \\
\hline 01N01E24CBD1 & 344033 & 905729 & $4 / 12 / 2002$ & 13.3 & $5 / 03 / 2006$ & 16.3 & -3.0 \\
\hline 01N02E01ADD1 & 344330 & 905016 & $4 / 17 / 2002$ & 25 & $5 / 02 / 2006$ & 28 & -3.0 \\
\hline 01N02E11BAB1 & 344255 & 905208 & $4 / 17 / 2002$ & 22 & $4 / 22 / 2006$ & 32 & -10.0 \\
\hline 01N02E12ABB1 & 344254 & 905040 & $4 / 10 / 2002$ & 27 & $5 / 02 / 2006$ & 27 & 0.0 \\
\hline 01N02E22CBA1 & 344056 & 905318 & $4 / 17 / 2002$ & 25 & $5 / 02 / 2006$ & 28.5 & -3.5 \\
\hline 01N02E33CBB1 & 343858 & 905434 & $4 / 12 / 2002$ & 11.5 & $5 / 02 / 2006$ & 16 & -4.5 \\
\hline 01N02E33CCB1 & 343851 & 905433 & $4 / 12 / 2002$ & 10 & $5 / 02 / 2006$ & 14 & -4.0 \\
\hline 01N03E02BBC1 & 344339 & 904601 & $3 / 14 / 2002$ & 53.46 & $3 / 21 / 2006$ & 48.57 & 4.9 \\
\hline 01N03E27ADD1 & 343952 & 904605 & $4 / 17 / 2002$ & 10.5 & $5 / 02 / 2006$ & 16 & -5.5 \\
\hline 01N03E35BBA1 & 343923 & 904549 & $3 / 14 / 2002$ & 12.87 & $3 / 21 / 2006$ & 10.24 & 2.6 \\
\hline 02N01E21BAA1 & 344633 & 910005 & $4 / 12 / 2002$ & 30.8 & $5 / 02 / 2006$ & 35.3 & -4.5 \\
\hline
\end{tabular}


Appendix 2. Information pertaining to the difference in water levels measured in 2002 and 2006 in the Mississippi River Valley alluvial aquifer in eastern Arkansas.-Continued

[Horizontal coordinate information is referenced to the North American Datum of 1983 (NAD83); *, control wells for duplicate measurements by USGS and NRCS]

\begin{tabular}{|c|c|c|c|c|c|c|c|}
\hline Station name & $\begin{array}{c}\text { Latitude } \\
\text { (degrees, } \\
\text { minutes, } \\
\text { seconds) }\end{array}$ & $\begin{array}{c}\text { Longitude } \\
\text { (degrees, } \\
\text { minutes, } \\
\text { seconds) }\end{array}$ & $\begin{array}{c}2002 \text { water- } \\
\text { level date }\end{array}$ & $\begin{array}{c}2002 \text { depth } \\
\text { to water } \\
\text { (feet below } \\
\text { land-surface } \\
\text { datum) }\end{array}$ & $\begin{array}{c}2006 \text { water- } \\
\text { level date }\end{array}$ & $\begin{array}{c}2006 \text { depth } \\
\text { to water } \\
\text { (feet below } \\
\text { land-surface } \\
\text { datum) }\end{array}$ & $\begin{array}{c}\text { Water-level } \\
\text { difference } \\
2002 \text { to } 2006 \\
\text { (feet) }\end{array}$ \\
\hline \multicolumn{8}{|c|}{ Lee County-Continued } \\
\hline 02N01E23BAA2 & 344632 & 905820 & $3 / 14 / 2002$ & 47.57 & $3 / 21 / 2006$ & 50.18 & -2.6 \\
\hline 02N01W12BAA1 & 344828 & 910330 & $3 / 20 / 2002$ & 40.55 & $3 / 21 / 2006$ & 43.52 & -3.0 \\
\hline 02N01W34DDC1 & 344410 & 910520 & $4 / 10 / 2002$ & 44.5 & $5 / 02 / 2006$ & 52 & -7.5 \\
\hline 02N02E08ADC1 & 344807 & 905339 & $3 / 14 / 2002$ & 40.01 & $3 / 21 / 2006$ & 43.46 & -3.5 \\
\hline 02N02E21ABC1 & 344622 & 905358 & $3 / 14 / 2002$ & 38.2 & $3 / 21 / 2006$ & 37.73 & 0.5 \\
\hline 02N02E22BBB1 & 344628 & 905327 & $4 / 10 / 2002$ & 32 & $5 / 02 / 2006$ & 28 & 4.0 \\
\hline 02N02E36DDC1 & 344355 & 905020 & $4 / 17 / 2002$ & 25 & $5 / 02 / 2006$ & 26.5 & -1.5 \\
\hline 02N03E08AAD1 & 344811 & 904838 & $3 / 20 / 2002$ & 44.45 & $3 / 21 / 2006$ & 43.89 & 0.6 \\
\hline 02N03E09DDD1 & 344723 & 904707 & $4 / 18 / 2002$ & 48.5 & $5 / 02 / 2006$ & 51 & -2.5 \\
\hline 02N03E29CAD1 & 344500 & 904846 & $4 / 10 / 2002$ & 44 & $5 / 02 / 2006$ & 50 & -6.0 \\
\hline 02N04E03ABD1 & 344855 & 903954 & $4 / 16 / 2002$ & 23 & $5 / 03 / 2006$ & 24.5 & -1.5 \\
\hline 02N04E15DAC1 & 344637 & 903950 & $3 / 20 / 2002$ & 18.57 & $3 / 21 / 2006$ & 17.62 & 1.0 \\
\hline 03N01E16CBA1 & 345222 & 910040 & $3 / 20 / 2002$ & 60.66 & $3 / 21 / 2006$ & 63.57 & -2.9 \\
\hline 03N01E32BCC1 & 344951 & 910150 & $4 / 10 / 2002$ & 59 & $5 / 02 / 2006$ & 62 & -3.0 \\
\hline 03N02E12CDC1 & 345239 & 905053 & $4 / 16 / 2002$ & 45 & $5 / 02 / 2006$ & 41 & 4.0 \\
\hline 03N02E13BBA1 & 345237 & 905107 & $3 / 20 / 2002$ & 48.76 & $3 / 22 / 2006$ & 49.98 & -1.2 \\
\hline 03N02E21CBC1 & 345111 & 905428 & $4 / 16 / 2002$ & 52.5 & $5 / 02 / 2006$ & 54 & -1.5 \\
\hline 03N02E29DAD1 & 345014 & 905430 & $3 / 14 / 2002$ & 43.13 & $3 / 21 / 2006$ & 42.71 & 0.4 \\
\hline 03N03E05CDD1 & 345327 & 904837 & $4 / 9 / 2002$ & 44 & $5 / 01 / 2006$ & 49.5 & -5.5 \\
\hline 03N03E18DAB1 & 345206 & 904919 & $4 / 16 / 2002$ & 30 & $5 / 02 / 2006$ & 29 & 1.0 \\
\hline 03N03E32CAB1 & 344933 & 904926 & $3 / 14 / 2002$ & 48.8 & $3 / 22 / 2006$ & 49.92 & -1.1 \\
\hline 03N04E07CBB1 & 345245 & 904312 & $4 / 16 / 2002$ & 31.5 & $5 / 01 / 2006$ & 30 & 1.5 \\
\hline 03N05E14DDA1 & 345148 & 903203 & $3 / 20 / 2002$ & 13.96 & $3 / 21 / 2006$ & 13.56 & 0.4 \\
\hline 03N05E26ADC1 & 345020 & 903215 & $4 / 16 / 2002$ & 6.5 & $5 / 03 / 2006$ & 7 & -0.5 \\
\hline \multicolumn{8}{|c|}{ Lincoln County } \\
\hline 07S06W03CCA2 & 340828 & 914114 & $4 / 19 / 2002$ & 13 & $4 / 12 / 2006$ & 19 & -6.0 \\
\hline 07S07W36CBD1 & 340411 & 914529 & $4 / 19 / 2002$ & 41 & $4 / 12 / 2006$ & 38 & 3.0 \\
\hline 08S04W06ABD1 & 340341 & 913116 & $4 / 19 / 2002$ & 17 & $4 / 12 / 2006$ & 20 & -3.0 \\
\hline 08S04W08BBB2 & 340254 & 913101 & $3 / 4 / 2002$ & 19.76 & $3 / 20 / 2006$ & 21.87 & -2.1 \\
\hline 08S04W29ABC1 & 340021 & 913044 & $4 / 19 / 2002$ & 42 & $4 / 12 / 2006$ & 41 & 1.0 \\
\hline 08S04W31CBA1 & 335901 & 913150 & $3 / 4 / 2002$ & 31.48 & $3 / 20 / 2006$ & 32.63 & -1.2 \\
\hline 08S05W12AAD1 & 340246 & 913214 & $4 / 19 / 2002$ & 21 & $4 / 12 / 2006$ & 22 & -1.0 \\
\hline 08S05W21DCD1 & 340027 & 913533 & $4 / 19 / 2002$ & 36 & $4 / 12 / 2006$ & 35 & 1.0 \\
\hline 08S05W32DCC1 & 335840 & 913644 & $4 / 19 / 2002$ & 44 & $4 / 12 / 2006$ & 43 & 1.0 \\
\hline 08S06W02ACB1 & 340339 & 913958 & $3 / 4 / 2002$ & 41.39 & $3 / 20 / 2006$ & 42.25 & -0.9 \\
\hline
\end{tabular}


Appendix 2. Information pertaining to the difference in water levels measured in 2002 and 2006 in the Mississippi River Valley alluvial aquifer in eastern Arkansas.-Continued

[Horizontal coordinate information is referenced to the North American Datum of 1983 (NAD83); *, control wells for duplicate measurements by USGS and NRCS]

\begin{tabular}{|c|c|c|c|c|c|c|c|}
\hline Station name & $\begin{array}{c}\text { Latitude } \\
\text { (degrees, } \\
\text { minutes, } \\
\text { seconds) }\end{array}$ & $\begin{array}{c}\text { Longitude } \\
\text { (degrees, } \\
\text { minutes, } \\
\text { seconds) }\end{array}$ & $\begin{array}{l}2002 \text { water- } \\
\text { level date }\end{array}$ & $\begin{array}{l}2002 \text { depth } \\
\text { to water } \\
\text { (feet below } \\
\text { land-surface } \\
\text { datum) }\end{array}$ & $\begin{array}{l}2006 \text { water- } \\
\text { level date }\end{array}$ & $\begin{array}{l}2006 \text { depth } \\
\text { to water } \\
\text { (feet below } \\
\text { land-surface } \\
\text { datum) }\end{array}$ & $\begin{array}{c}\text { Water-level } \\
\text { difference } \\
2002 \text { to } 2006 \\
\text { (feet) }\end{array}$ \\
\hline \multicolumn{8}{|c|}{ Lincoln County-Continued } \\
\hline 08S07W05DDD1 & 340301 & 914903 & $3 / 2 / 2002$ & 28.68 & $3 / 20 / 2006$ & 29.96 & -1.3 \\
\hline 09S04W06CBB1 & 335721 & 913252 & $4 / 19 / 2002$ & 32 & $4 / 12 / 2006$ & 40 & -8.0 \\
\hline 09S05W14ABC1 & 335553 & 913439 & $3 / 4 / 2002$ & 36.62 & $3 / 20 / 2006$ & 37.21 & -0.6 \\
\hline 09S05W17BCB1 & 335552 & 913820 & $3 / 4 / 2002$ & 39.69 & $3 / 20 / 2006$ & 41.09 & -1.4 \\
\hline 09S05W19CCC1 & 335428 & 913941 & $4 / 19 / 2002$ & 34 & $4 / 12 / 2006$ & 31 & 3.0 \\
\hline 09S06W04BCD1 & 335821 & 914346 & $3 / 4 / 2002$ & 38.85 & $3 / 20 / 2006$ & 38.12 & 0.7 \\
\hline 09S06W04BDD1 & 335759 & 914335 & $4 / 19 / 2002$ & 38 & $4 / 12 / 2006$ & 36 & 2.0 \\
\hline 09S06W23CDB1 & 335440 & 914136 & $3 / 4 / 2002$ & 29.27 & $3 / 20 / 2006$ & 29.73 & -0.5 \\
\hline 10S05W06DCC1 & 335155 & 913908 & $3 / 4 / 2002$ & 28.92 & $3 / 20 / 2006$ & 29.92 & -1.0 \\
\hline \multicolumn{8}{|c|}{ Lonoke County } \\
\hline 01N08W03DDA1 & 344411 & 915050 & $4 / 19 / 2002$ & 129.5 & $4 / 17 / 2006$ & 139.2 & -9.7 \\
\hline 01N09W07DAA1 & 344337 & 920030 & $4 / 17 / 2002$ & 49.2 & $4 / 17 / 2006$ & 47 & 2.2 \\
\hline 01N09W13DAB1 & 344235 & 915517 & $4 / 2 / 2002$ & 85.75 & $4 / 13 / 2006$ & 86.74 & -1.0 \\
\hline 01N10W15CDA1 & 344236 & 920415 & $4 / 17 / 2002$ & 30.8 & $4 / 17 / 2006$ & 24.7 & 6.1 \\
\hline 01S06W31ABB1 & 343459 & 914131 & $3 / 18 / 2002$ & 78.37 & $4 / 13 / 2006$ & 78.73 & -0.4 \\
\hline 01S06W32BBB1 & 343501 & 914056 & $4 / 17 / 2002$ & 79.3 & $4 / 17 / 2006$ & 77 & 2.3 \\
\hline 01S07W12ABA1 & 343834 & 914230 & $3 / 18 / 2002$ & 67.3 & $4 / 13 / 2006$ & 69.93 & -2.6 \\
\hline 01S08W24CDD1 & 343606 & 914912 & $3 / 18 / 2002$ & 79.35 & $4 / 13 / 2006$ & 81 & -1.7 \\
\hline 01S09W02DDD1 & 343857 & 915624 & $4 / 17 / 2002$ & 88.5 & $4 / 17 / 2006$ & 83 & 5.5 \\
\hline 01S09W36CCC1 & 343435 & 915619 & $3 / 18 / 2002$ & 60.76 & $4 / 13 / 2006$ & 62.51 & -1.8 \\
\hline 01S10W01ACB1 & 343927 & 920215 & $3 / 18 / 2002$ & 46 & 4/13/2006 & 45.92 & 0.1 \\
\hline 02N07W07DAA1 & 344845 & 914707 & $4 / 17 / 2002$ & 132.6 & $4 / 17 / 2006$ & 140.2 & -7.6 \\
\hline 02N07W16BAB1 & 344815 & 914540 & $3 / 22 / 2002$ & 135.09 & $4 / 13 / 2006$ & 137.64 & -2.5 \\
\hline 02N08W16ABC1 & 344806 & 915114 & $3 / 22 / 2002$ & 118.4 & $4 / 13 / 2006$ & 128.39 & -10.0 \\
\hline 02N08W23CAB1 & 344659 & 915118 & $4 / 19 / 2002$ & 133.5 & $4 / 17 / 2006$ & 129.7 & 3.8 \\
\hline 02N09W02BDB1 & 344955 & 915841 & $4 / 2 / 2002$ & 118.84 & $4 / 13 / 2006$ & 126.04 & -7.2 \\
\hline 02S07W05CDC1 & 343326 & 914715 & $4 / 19 / 2002$ & 66.9 & $4 / 17 / 2006$ & 70.4 & -3.5 \\
\hline 02S07W10CCB1 & 343246 & 914525 & $3 / 8 / 2002$ & 61.03 & $4 / 13 / 2006$ & 62.39 & -1.4 \\
\hline 02S07W20ACD1 & 343112 & 914655 & $4 / 19 / 2002$ & 58.7 & $4 / 17 / 2006$ & 60.6 & -1.9 \\
\hline 02S08W13BBB1 & 343232 & 914935 & $3 / 8 / 2002$ & 57.08 & $4 / 13 / 2006$ & 59.85 & -2.8 \\
\hline 02S08W34DBB1 & 343003 & 915150 & $3 / 8 / 2002$ & 60.67 & $4 / 13 / 2006$ & 66.62 & -6.0 \\
\hline 02S09W30CDD1 & 343014 & 920116 & $3 / 8 / 2002$ & 36.56 & $4 / 13 / 2006$ & 39.44 & -2.9 \\
\hline 03N07W08BDB1 & 345407 & 914638 & $4 / 16 / 2002$ & 92.71 & 2/09/2006 & 96.48 & -3.8 \\
\hline 03N07W15DBC2 & 345253 & 914417 & $3 / 22 / 2002$ & 79.22 & $4 / 13 / 2006$ & 82.43 & -3.2 \\
\hline 03N07W29ADA1 & 345129 & 914558 & $4 / 16 / 2002$ & 87.3 & $2 / 09 / 2006$ & 94.22 & -6.9 \\
\hline
\end{tabular}


Appendix 2. Information pertaining to the difference in water levels measured in 2002 and 2006 in the Mississippi River Valley alluvial aquifer in eastern Arkansas.-Continued

[Horizontal coordinate information is referenced to the North American Datum of 1983 (NAD83); *, control wells for duplicate measurements by USGS and NRCS]

\begin{tabular}{|c|c|c|c|c|c|c|c|}
\hline Station name & $\begin{array}{l}\text { Latitude } \\
\text { (degrees, } \\
\text { minutes, } \\
\text { seconds) }\end{array}$ & $\begin{array}{c}\text { Longitude } \\
\text { (degrees, } \\
\text { minutes, } \\
\text { seconds) }\end{array}$ & $\begin{array}{l}2002 \text { water- } \\
\text { level date }\end{array}$ & $\begin{array}{c}2002 \text { depth } \\
\text { to water } \\
\text { (feet below } \\
\text { land-surface } \\
\text { datum) }\end{array}$ & $\begin{array}{l}2006 \text { water- } \\
\text { level date }\end{array}$ & $\begin{array}{l}2006 \text { depth } \\
\text { to water } \\
\text { (feet below } \\
\text { land-surface } \\
\text { datum) }\end{array}$ & $\begin{array}{c}\text { Water-level } \\
\text { difference } \\
2002 \text { to } 2006 \\
\text { (feet) }\end{array}$ \\
\hline \multicolumn{8}{|c|}{ Lonoke County-Continued } \\
\hline 03N07W35CDC2 & 344957 & 914332 & $3 / 22 / 2002$ & 113.25 & $4 / 13 / 2006$ & 116.1 & -2.8 \\
\hline 03N08W03BAA1 & 345519 & 915054 & $4 / 15 / 2002$ & 88.18 & $2 / 09 / 2006$ & 94.87 & -6.7 \\
\hline 03N08W03CCC1 & 345430 & 915123 & $4 / 15 / 2002$ & 96.38 & $2 / 09 / 2006$ & 101.86 & -5.5 \\
\hline 03N08W05CCC1 & 345429 & 915323 & $4 / 15 / 2002$ & 77.23 & 2/09/2006 & 81.08 & -3.8 \\
\hline 03N08W08ABA1 & 345427 & 915248 & $4 / 15 / 2002$ & 90.43 & $2 / 09 / 2006$ & 93.11 & -2.7 \\
\hline 03N08W10ACB1 & 345415 & 915053 & $4 / 15 / 2002$ & 84.12 & 2/09/2006 & 91.04 & -6.9 \\
\hline 03N08W10ADD1 & 345401 & 915023 & $4 / 15 / 2002$ & 82.29 & $2 / 10 / 2006$ & 89.88 & -7.6 \\
\hline 03N08W11ABD1 & 345419 & 914936 & $4 / 16 / 2002$ & 97.5 & $2 / 10 / 2006$ & 103.86 & -6.4 \\
\hline 03N08W11ACA1 & 345413 & 914934 & $4 / 17 / 2002$ & 94.92 & $2 / 10 / 2006$ & 101.53 & -6.6 \\
\hline 03N08W21BCC1 & 345220 & 915220 & $3 / 22 / 2002$ & 79.35 & $4 / 13 / 2006$ & 81.66 & -2.3 \\
\hline 03N08W29BBB1 & 345147 & 915333 & $4 / 15 / 2002$ & 108.95 & 2/09/2006 & 112.33 & -3.4 \\
\hline 03N08W29BCC1 & 345125 & 915333 & $4 / 15 / 2002$ & 124.06 & 2/09/2006 & 128.81 & -4.8 \\
\hline 03N08W32ABB2 & 345057 & 915259 & $3 / 19 / 2002$ & 115.68 & $4 / 13 / 2006$ & 118.68 & -3.0 \\
\hline 03N08W34ADD1 & 345035 & 915028 & $4 / 15 / 2002$ & 113.3 & 2/09/2006 & 122.5 & -9.2 \\
\hline 04N08W05ACA1 & 350020 & 915247 & $4 / 16 / 2002$ & 44.08 & $2 / 10 / 2006$ & 45.64 & -1.6 \\
\hline 04N08W10BDD1 & 345917 & 915055 & $4 / 15 / 2002$ & 24.56 & $2 / 10 / 2006$ & 26.15 & -1.6 \\
\hline 04N08W16DCC1 & 345757 & 915154 & $4 / 15 / 2002$ & 43.18 & $2 / 10 / 2006$ & 46.78 & -3.6 \\
\hline 04N08W19BBB1 & 345753 & 915432 & $4 / 15 / 2002$ & 5.27 & $2 / 10 / 2006$ & 12.47 & -7.2 \\
\hline 04N08W26AAD1 & 345652 & 914917 & $4 / 15 / 2002$ & 66.89 & 2/09/2006 & 72.86 & -6.0 \\
\hline 04N08W28CAC1 & 345620 & 915216 & $4 / 15 / 2002$ & 50.88 & 2/09/2006 & 55 & -4.1 \\
\hline 04N08W28CAD1 & 345626 & 915204 & $4 / 15 / 2002$ & 66.36 & $2 / 09 / 2006$ & 70.26 & -3.9 \\
\hline 04N08W28CCC1 & 345615 & 915225 & $4 / 15 / 2002$ & 56.53 & 2/09/2006 & 60.39 & -3.9 \\
\hline 04N08W31CBB2 & 345547 & 915439 & $4 / 15 / 2002$ & 30.42 & 2/09/2006 & 28.68 & 1.7 \\
\hline 04N08W36DBB1 & 345541 & 914914 & $4 / 15 / 2002$ & 87.5 & 2/09/2006 & 93.71 & -6.2 \\
\hline \multicolumn{8}{|c|}{ Mississippi County } \\
\hline 10N08E21ABA1 & 352852 & 901415 & $4 / 10 / 2002$ & 26.4 & $4 / 18 / 2006$ & 25 & 1.4 \\
\hline 10N08E21BDC1 & 352830 & 901407 & $4 / 10 / 2002$ & 25 & $4 / 18 / 2006$ & 25 & 0.0 \\
\hline 10N08E22ABA2 & 352851 & 901312 & $3 / 26 / 2002$ & 23.2 & $4 / 20 / 2006$ & 24.04 & -0.8 \\
\hline 10N09E08ACC1 & 352949 & 900926 & $3 / 26 / 2002$ & 14.64 & $4 / 20 / 2006$ & 15.84 & -1.2 \\
\hline 11N09E34BBB1 & 353218 & 900715 & $3 / 26 / 2002$ & 17.12 & $4 / 20 / 2006$ & 16.7 & 0.4 \\
\hline 11N10E09BCB1 & 353530 & 900202 & $4 / 10 / 2002$ & 14.8 & $4 / 14 / 2006$ & 20 & -5.2 \\
\hline 12N08E08BCB1 & 354047 & 901559 & $3 / 26 / 2002$ & 6.44 & $4 / 20 / 2006$ & 10.07 & -3.6 \\
\hline 12N08E28DDB1 & 353707 & 901406 & $4 / 10 / 2002$ & 12 & $4 / 18 / 2006$ & 20 & -8.0 \\
\hline $12 \mathrm{~N} 09 \mathrm{E} 12 \mathrm{ABC} 1$ & 354054 & 900449 & $4 / 11 / 2002$ & 8.7 & $4 / 14 / 2006$ & 17 & -8.3 \\
\hline 12N10E04CAA1 & 354124 & 900136 & $4 / 11 / 2002$ & 9.4 & $4 / 14 / 2006$ & 20 & -10.6 \\
\hline
\end{tabular}


Appendix 2. Information pertaining to the difference in water levels measured in 2002 and 2006 in the Mississippi River Valley alluvial aquifer in eastern Arkansas. - Continued

[Horizontal coordinate information is referenced to the North American Datum of 1983 (NAD83); *, control wells for duplicate measurements by USGS and NRCS]

\begin{tabular}{|c|c|c|c|c|c|c|c|}
\hline Station name & $\begin{array}{c}\text { Latitude } \\
\text { (degrees, } \\
\text { minutes, } \\
\text { seconds) }\end{array}$ & $\begin{array}{c}\text { Longitude } \\
\text { (degrees, } \\
\text { minutes, } \\
\text { seconds) }\end{array}$ & $\begin{array}{c}2002 \text { water- } \\
\text { level date }\end{array}$ & $\begin{array}{c}2002 \text { depth } \\
\text { to water } \\
\text { (feet below } \\
\text { land-surface } \\
\text { datum) }\end{array}$ & $\begin{array}{l}2006 \text { water- } \\
\text { level date }\end{array}$ & $\begin{array}{c}2006 \text { depth } \\
\text { to water } \\
\text { (feet below } \\
\text { land-surface } \\
\text { datum) }\end{array}$ & $\begin{array}{c}\text { Water-level } \\
\text { difference } \\
2002 \text { to } 2006 \\
\text { (feet) }\end{array}$ \\
\hline \multicolumn{8}{|c|}{ Mississippi County_Continued } \\
\hline 12N10E07BCD1 & 354036 & 900404 & $4 / 11 / 2002$ & 11.5 & $4 / 14 / 2006$ & 22 & -10.5 \\
\hline 12N10E21DBA1 & 353842 & 900122 & $4 / 10 / 2002$ & 14.9 & $4 / 14 / 2006$ & 17 & -2.1 \\
\hline 13N08E24ABB1 & 354428 & 901112 & $4 / 10 / 2002$ & 11 & $4 / 18 / 2006$ & 9 & 2.0 \\
\hline 13N09E30CCD1 & 354248 & 901029 & $3 / 25 / 2002$ & 6.91 & $4 / 20 / 2006$ & 12.88 & -6.0 \\
\hline 13N10E34DBB1 & 354218 & 900024 & $3 / 25 / 2002$ & 6.1 & $4 / 20 / 2006$ & 8.58 & -2.5 \\
\hline 14N08E12DAB1 & 355104 & 901052 & $3 / 25 / 2002$ & 3.85 & $4 / 19 / 2006$ & 8.38 & -4.5 \\
\hline 14N08E20DAA1 & 354921 & 901458 & $4 / 10 / 2002$ & 4 & $4 / 18 / 2006$ & 5 & -1.0 \\
\hline 14N08E26CC1 & 354803 & 901235 & $4 / 10 / 2002$ & 4 & $4 / 18 / 2006$ & 5 & -1.0 \\
\hline 14N10E18ABC1 & 355022 & 900345 & $3 / 25 / 2002$ & 9.25 & $4 / 19 / 2006$ & 13.02 & -3.8 \\
\hline 14N11E03BCB1 & 355158 & 895433 & $3 / 25 / 2002$ & 3.87 & $4 / 20 / 2006$ & 5.26 & -1.4 \\
\hline 14N11E17CCB1 & 354955 & 895639 & $4 / 11 / 2002$ & 7.9 & $4 / 10 / 2006$ & 8 & -0.1 \\
\hline 14N11E33CAA1 & 354727 & 895508 & $4 / 11 / 2002$ & 7.8 & $4 / 10 / 2006$ & 15 & -7.2 \\
\hline 15N08E08DBC2 & 355605 & 901526 & $3 / 26 / 2002$ & 7.87 & $4 / 19 / 2006$ & 11.97 & -4.1 \\
\hline 15N10E21ABC1 & 355447 & 900135 & $4 / 10 / 2002$ & 9 & $4 / 13 / 2006$ & 13 & -4.0 \\
\hline 15N12E01BCD1 & 355704 & 894601 & $4 / 11 / 2002$ & 12.6 & $4 / 13 / 2006$ & 11 & 1.6 \\
\hline 16N10E28BBD1* & 355906 & 900156 & $4 / 10 / 2002$ & 8 & $4 / 13 / 2006$ & 14.5 & -6.5 \\
\hline 16N10E28BBD1* & 355906 & 900156 & $3 / 25 / 2002$ & 5.28 & $4 / 20 / 2006$ & 11.35 & -6.1 \\
\hline 16N11E23ADA1 & 355947 & 895231 & $3 / 25 / 2002$ & 10.1 & $4 / 20 / 2006$ & 12.7 & -2.6 \\
\hline \multicolumn{8}{|c|}{ Monroe County } \\
\hline 01N01W21CDC2 & 344037 & 910707 & $3 / 13 / 2002$ & 33.87 & $4 / 04 / 2006$ & 36.57 & -2.7 \\
\hline 01N02W12CBC1 & 344242 & 911032 & $5 / 1 / 2002$ & 36.21 & $4 / 04 / 2006$ & 39.21 & -3.0 \\
\hline 01N03W23BAC1 & 344124 & 911743 & $4 / 10 / 2002$ & 13 & $4 / 14 / 2006$ & 16 & -3.0 \\
\hline 01N03W24BBB1 & 344135 & 911651 & $3 / 13 / 2002$ & 31.11 & $4 / 04 / 2006$ & 28.63 & 2.5 \\
\hline 01N04W33BBB2 & 343960 & 912649 & $3 / 13 / 2002$ & 93.82 & $4 / 04 / 2006$ & 95.15 & -1.3 \\
\hline 01S01W13CDD1 & 343611 & 910341 & $3 / 12 / 2002$ & 20.08 & $4 / 04 / 2006$ & 20.37 & -0.3 \\
\hline 01S01W16DB & 343615 & 910632 & $4 / 10 / 2002$ & 16.5 & $4 / 14 / 2006$ & 22 & -5.5 \\
\hline 01S01W18DCD1 & 343618 & 910849 & $3 / 13 / 2002$ & 23.25 & $4 / 04 / 2006$ & 23.05 & 0.2 \\
\hline 01S02W20BBB $1 *$ & 343613 & 911456 & $4 / 10 / 2002$ & 11 & $4 / 14 / 2006$ & 12 & -1.0 \\
\hline 01S02W20BBB $1 *$ & 343613 & 911456 & $3 / 12 / 2002$ & 11.57 & $4 / 04 / 2006$ & 13.35 & -1.8 \\
\hline 01S03W20BBA1* & 343538 & 912118 & $4 / 10 / 2002$ & 78.5 & $4 / 14 / 2006$ & 79 & -0.5 \\
\hline 01S03W20BBA1* & 343538 & 912118 & $3 / 13 / 2002$ & 73.16 & $4 / 04 / 2006$ & 73.73 & -0.6 \\
\hline 01S04W01BAB1 & 343906 & 912317 & $3 / 13 / 2002$ & 76.91 & $4 / 04 / 2006$ & 68.7 & 8.2 \\
\hline 02N01W19ADD1 & 344624 & 910814 & $4 / 17 / 2002$ & 48 & $4 / 14 / 2006$ & 53 & -5.0 \\
\hline 02N01W19BBA1 & 344645 & 910912 & $3 / 13 / 2002$ & 51.15 & $4 / 05 / 2006$ & 54.27 & -3.1 \\
\hline 02N03W35BCA1 & 344455 & 911745 & $4 / 10 / 2002$ & 29 & $4 / 14 / 2006$ & 34 & -5.0 \\
\hline
\end{tabular}


Appendix 2. Information pertaining to the difference in water levels measured in 2002 and 2006 in the Mississippi River Valley alluvial aquifer in eastern Arkansas.-Continued

[Horizontal coordinate information is referenced to the North American Datum of 1983 (NAD83); *, control wells for duplicate measurements by USGS and NRCS]

\begin{tabular}{|c|c|c|c|c|c|c|c|}
\hline Station name & $\begin{array}{c}\text { Latitude } \\
\text { (degrees, } \\
\text { minutes, } \\
\text { seconds) }\end{array}$ & $\begin{array}{c}\text { Longitude } \\
\text { (degrees, } \\
\text { minutes, } \\
\text { seconds) }\end{array}$ & $\begin{array}{c}2002 \text { water- } \\
\text { level date }\end{array}$ & $\begin{array}{c}2002 \text { depth } \\
\text { to water } \\
\text { (feet below } \\
\text { land-surface } \\
\text { datum) }\end{array}$ & $\begin{array}{c}2006 \text { water- } \\
\text { level date }\end{array}$ & $\begin{array}{c}2006 \text { depth } \\
\text { to water } \\
\text { (feet below } \\
\text { land-surface } \\
\text { datum) }\end{array}$ & $\begin{array}{c}\text { Water-level } \\
\text { difference } \\
2002 \text { to } 2006 \\
\text { (feet) }\end{array}$ \\
\hline \multicolumn{8}{|c|}{ Monroe County-Continued } \\
\hline 02S01W01BCD1 & 343305 & 910408 & $4 / 10 / 2002$ & 20 & $4 / 14 / 2006$ & 19 & 1.0 \\
\hline 02S02W01BCA1 & 343322 & 911031 & $3 / 13 / 2002$ & 11.54 & $4 / 04 / 2006$ & 12.13 & -0.6 \\
\hline 02S02W11DAC1 & 343209 & 911101 & $5 / 1 / 2002$ & 6.53 & $4 / 04 / 2006$ & 10.16 & -3.6 \\
\hline 03N01W20ABA1 & 345201 & 910723 & $3 / 13 / 2002$ & 46.28 & $4 / 05 / 2006$ & 48.07 & -1.8 \\
\hline 03N02W31ADC1 & 344958 & 911447 & $3 / 13 / 2002$ & 37.9 & $4 / 05 / 2006$ & 38.53 & -0.6 \\
\hline 03N03W36AAA1 & 345027 & 911547 & $3 / 13 / 2002$ & 18.91 & $4 / 05 / 2006$ & 22.2 & -3.3 \\
\hline 04N02W01BCC1 & 345929 & 911004 & $4 / 17 / 2002$ & 37 & $4 / 13 / 2006$ & 39.5 & -2.5 \\
\hline 04N02W05BBB 1 & 345957 & 911311 & $4 / 17 / 2002$ & 13 & $4 / 13 / 2006$ & 15 & -2.0 \\
\hline 04N02W27CDD3 & 345540 & 911150 & $3 / 13 / 2002$ & 45.15 & $4 / 05 / 2006$ & 45.5 & -0.4 \\
\hline 04N02W28DDD3 & 345535 & 911221 & $3 / 13 / 2002$ & 32.82 & $4 / 05 / 2006$ & 32.19 & 0.6 \\
\hline 04N02W30BBB 1 & 345628 & 911525 & $3 / 13 / 2002$ & 14.9 & $4 / 05 / 2006$ & 14.53 & 0.4 \\
\hline \multicolumn{8}{|c|}{ Phillips County } \\
\hline 01S01E20DDB1 & 343529 & 910058 & $4 / 16 / 2002$ & 16.8 & $4 / 10 / 2006$ & 26 & -9.2 \\
\hline 01S02E09CBB1* & 343719 & 905434 & $4 / 16 / 2002$ & 9.5 & $4 / 10 / 2006$ & 14.8 & -5.3 \\
\hline 01S02E09CBB1* & 343719 & 905434 & $3 / 14 / 2002$ & 9.49 & $3 / 21 / 2006$ & 14.29 & -4.8 \\
\hline 01S02E32BCC1 & 343350 & 905526 & $4 / 16 / 2002$ & 31.6 & $4 / 10 / 2006$ & 37 & -5.4 \\
\hline 01S03E02ADD1 & 343814 & 904511 & $4 / 16 / 2002$ & 14.7 & $4 / 10 / 2006$ & 16.6 & -1.9 \\
\hline 01S03E10ABB1 & 343741 & 904634 & $4 / 16 / 2002$ & 13 & $4 / 10 / 2006$ & 18 & -5.0 \\
\hline 01S03E20BDD1 & 343533 & 904846 & $4 / 16 / 2002$ & 29 & $4 / 10 / 2006$ & 33 & -4.0 \\
\hline 01S04E05DCD1* & 343802 & 904151 & $4 / 16 / 2002$ & 43 & $3 / 21 / 2006$ & 48.91 & -5.9 \\
\hline 01S04E05DCD1* & 343802 & 904151 & $5 / 1 / 2002$ & 47.2 & $4 / 10 / 2006$ & 49 & -1.8 \\
\hline 02S01E28CCB1 & 342916 & 910058 & $3 / 14 / 2002$ & 18.37 & $3 / 21 / 2006$ & 17.95 & 0.4 \\
\hline 02S02E29DDD1 & 342901 & 905444 & $4 / 16 / 2002$ & 23.5 & $4 / 10 / 2006$ & 27.6 & -4.1 \\
\hline 02S02E33ACC1 & 342824 & 905412 & $4 / 16 / 2002$ & 22.7 & $4 / 10 / 2006$ & 26 & -3.3 \\
\hline 02S03E15ACD1 & 343110 & 904621 & $3 / 14 / 2002$ & 11.49 & $5 / 08 / 2006$ & 13.78 & -2.3 \\
\hline 02S03E34BCD1 & 342828 & 904653 & $4 / 18 / 2002$ & 18.4 & $4 / 12 / 2006$ & 18 & 0.4 \\
\hline 02S04E27AAC1* & 342932 & 904001 & $4 / 18 / 2002$ & 8.5 & $4 / 10 / 2006$ & 10 & -1.5 \\
\hline 02S04E27AAC1* & 342932 & 904001 & $3 / 14 / 2002$ & 8 & $3 / 21 / 2006$ & 8.59 & -0.6 \\
\hline 03S02E35DDA1 & 342256 & 905130 & $3 / 14 / 2002$ & 20.71 & $3 / 21 / 2006$ & 21.23 & -0.5 \\
\hline 03S03E04DAA1 & 342735 & 904710 & $5 / 1 / 2002$ & 19.72 & $3 / 21 / 2006$ & 19.29 & 0.4 \\
\hline 03S04E02CAA1* & 342732 & 903918 & $4 / 18 / 2002$ & 11 & $4 / 10 / 2006$ & 17 & -6.0 \\
\hline 03S04E02CAA1* & 342732 & 903918 & $5 / 1 / 2002$ & 12 & $3 / 21 / 2006$ & 16.16 & -4.2 \\
\hline 04S01E01AAD1 & 342238 & 905700 & $4 / 18 / 2002$ & 17 & $4 / 12 / 2006$ & 22 & -5.0 \\
\hline 04S01E14CDD1 & 342014 & 905837 & $4 / 18 / 2002$ & 12.1 & $4 / 12 / 2006$ & 15 & -2.9 \\
\hline 04S01E23CCA1 & 341931 & 905853 & $3 / 14 / 2002$ & 13.45 & $3 / 21 / 2006$ & 13.4 & 0.0 \\
\hline
\end{tabular}


Appendix 2. Information pertaining to the difference in water levels measured in 2002 and 2006 in the Mississippi River Valley alluvial aquifer in eastern Arkansas.-Continued

[Horizontal coordinate information is referenced to the North American Datum of 1983 (NAD83); *, control wells for duplicate measurements by USGS and NRCS]

\begin{tabular}{|c|c|c|c|c|c|c|c|}
\hline Station name & $\begin{array}{l}\text { Latitude } \\
\text { (degrees, } \\
\text { minutes, } \\
\text { seconds) }\end{array}$ & $\begin{array}{c}\text { Longitude } \\
\text { (degrees, } \\
\text { minutes, } \\
\text { seconds) }\end{array}$ & $\begin{array}{l}2002 \text { water- } \\
\text { level date }\end{array}$ & $\begin{array}{l}2002 \text { depth } \\
\text { to water } \\
\text { (feet below } \\
\text { land-surface } \\
\text { datum) }\end{array}$ & $\begin{array}{l}2006 \text { water- } \\
\text { level date }\end{array}$ & $\begin{array}{l}2006 \text { depth } \\
\text { to water } \\
\text { (feet below } \\
\text { land-surface } \\
\text { datum) }\end{array}$ & $\begin{array}{l}\text { Water-level } \\
\text { difference } \\
2002 \text { to } 2006 \\
\text { (feet) }\end{array}$ \\
\hline \multicolumn{8}{|c|}{ Phillips County-Continued } \\
\hline 04S02E01DBB1 & 342220 & 905053 & $4 / 18 / 2002$ & 12.2 & $4 / 12 / 2006$ & 15.6 & -3.4 \\
\hline 05S02E18BDA1 & 341535 & 905628 & $3 / 14 / 2002$ & 17.47 & $3 / 21 / 2006$ & 22.5 & -5.0 \\
\hline \multicolumn{8}{|c|}{ Poinsett County } \\
\hline 10N01E02AAA & 353205 & 905654 & $4 / 4 / 2002$ & 93 & $4 / 13 / 2006$ & 98 & -5.0 \\
\hline 10N01E14CC1 & 352910 & 905814 & $3 / 25 / 2002$ & 87.48 & $3 / 28 / 2006$ & 92.41 & -4.9 \\
\hline 10N01E16CCB1 & 352922 & 910005 & $3 / 25 / 2002$ & 70.82 & $3 / 28 / 2006$ & 74.99 & -4.2 \\
\hline 10N01E32CBB1 & 352657 & 910053 & $4 / 4 / 2002$ & 50 & $4 / 13 / 2006$ & 74 & -24.0 \\
\hline 10N01E33ACB1 & 352746 & 905931 & $4 / 4 / 2002$ & 74 & $4 / 13 / 2006$ & 77 & -3.0 \\
\hline 10N02E13BCC1 & 352949 & 905026 & $3 / 25 / 2002$ & 99.21 & $3 / 28 / 2006$ & 103.24 & -4.0 \\
\hline 10N02E20BAB1 & 352906 & 905418 & $4 / 4 / 2002$ & 99 & $4 / 13 / 2006$ & 112.5 & -13.5 \\
\hline 10N03E14DAB1 & 352947 & 904405 & $3 / 25 / 2002$ & 114.62 & $3 / 28 / 2006$ & 118.63 & -4.0 \\
\hline 10N03E35CDD1 & 352656 & 904436 & $3 / 25 / 2002$ & 121.67 & $3 / 28 / 2006$ & 124.43 & -2.8 \\
\hline 10N04E35BBA1 & 352745 & 903831 & $4 / 3 / 2002$ & 20 & $4 / 17 / 2006$ & 21 & -1.0 \\
\hline 10N05E15BDD1 & 352937 & 903253 & $3 / 26 / 2002$ & 11.45 & $3 / 29 / 2006$ & 14.76 & -3.3 \\
\hline 10N07E22AAC1 & 352847 & 901935 & $3 / 26 / 2002$ & 28.2 & $3 / 29 / 2006$ & 27.8 & 0.4 \\
\hline 11N01E17DDC1 & 353437 & 910015 & 4/4/2002 & 76 & $4 / 13 / 2006$ & 78 & -2.0 \\
\hline 11N01E17DDD1 & 353437 & 910013 & $3 / 25 / 2002$ & 74.6 & $3 / 28 / 2006$ & 78.61 & -4.0 \\
\hline 11N01E26AA1 & 353340 & 905653 & $3 / 25 / 2002$ & 92.03 & $3 / 28 / 2006$ & 94.72 & -2.7 \\
\hline 11N01E34AAA & 353256 & 905759 & $4 / 4 / 2002$ & 84 & $4 / 13 / 2006$ & 88.5 & -4.5 \\
\hline 11N02E26AAB1 & 353350 & 905034 & $3 / 25 / 2002$ & 104.07 & $3 / 28 / 2006$ & 107.55 & -3.5 \\
\hline 11N02E30BBB1 & 353352 & 905540 & $4 / 4 / 2002$ & 98 & 4/17/2006 & 102.5 & -4.5 \\
\hline 11N02E34CBA1 & 353238 & 905222 & $4 / 4 / 2002$ & 94 & $4 / 13 / 2006$ & 109 & -15.0 \\
\hline 11N03E10DDA1 & 353546 & 904457 & $3 / 25 / 2002$ & 102 & $3 / 28 / 2006$ & 104.78 & -2.8 \\
\hline 11N03E18BAB1 & 353538 & 904852 & $3 / 25 / 2002$ & 101.5 & $3 / 28 / 2006$ & 105.34 & -3.8 \\
\hline 11N04E36ABA1 & 353251 & 903654 & $4 / 3 / 2002$ & 19 & $4 / 17 / 2006$ & 18 & 1.0 \\
\hline 11N07E18CAB1 & 353435 & 902320 & $3 / 26 / 2002$ & 14.34 & $3 / 29 / 2006$ & 15.37 & -1.0 \\
\hline 12N01E07CDA1 & 354054 & 910141 & $3 / 25 / 2002$ & 52.5 & $3 / 28 / 2006$ & 53.97 & -1.5 \\
\hline 12N01E22DAB1 & 353922 & 905809 & 4/4/2002 & 72 & $4 / 13 / 2006$ & 74 & -2.0 \\
\hline 12N02E25DCC1 & 353820 & 904944 & $4 / 4 / 2002$ & 108 & 4/13/2006 & 112 & -4.0 \\
\hline 12N02E34CCC1 & 353724 & 905230 & $4 / 4 / 2002$ & 109 & $4 / 13 / 2006$ & 112.5 & -3.5 \\
\hline 12N03E01CBD1 & 354154 & 904329 & 4/4/2002 & 91 & 4/17/2006 & 93 & -2.0 \\
\hline 12N03E04DAD1* & 354158 & 904600 & $4 / 4 / 2002$ & 102 & $4 / 17 / 2006$ & 104 & -2.0 \\
\hline 12N03E04DAD1* & 354158 & 904600 & $3 / 25 / 2002$ & 100.81 & $3 / 28 / 2006$ & 103.52 & -2.7 \\
\hline 12N03E36ACB1 & 353749 & 904319 & $3 / 25 / 2002$ & 95.66 & $3 / 28 / 2006$ & 98.57 & -2.9 \\
\hline 12N04E08CDA & 354053 & 904112 & $4 / 4 / 2002$ & 86 & 4/17/2006 & 88 & -2.0 \\
\hline
\end{tabular}


Appendix 2. Information pertaining to the difference in water levels measured in 2002 and 2006 in the Mississippi River Valley alluvial aquifer in eastern Arkansas.-Continued

[Horizontal coordinate information is referenced to the North American Datum of 1983 (NAD83); *, control wells for duplicate measurements by USGS and NRCS]

\begin{tabular}{|c|c|c|c|c|c|c|c|}
\hline Station name & $\begin{array}{c}\text { Latitude } \\
\text { (degrees, } \\
\text { minutes, } \\
\text { seconds) }\end{array}$ & $\begin{array}{c}\text { Longitude } \\
\text { (degrees, } \\
\text { minutes, } \\
\text { seconds) }\end{array}$ & $\begin{array}{l}2002 \text { water- } \\
\text { level date }\end{array}$ & $\begin{array}{c}2002 \text { depth } \\
\text { to water } \\
\text { (feet below } \\
\text { land-surface } \\
\text { datum) }\end{array}$ & $\begin{array}{l}2006 \text { water- } \\
\text { level date }\end{array}$ & $\begin{array}{c}2006 \text { depth } \\
\text { to water } \\
\text { (feet below } \\
\text { land-surface } \\
\text { datum) }\end{array}$ & $\begin{array}{c}\text { Water-level } \\
\text { difference } \\
2002 \text { to } 2006 \\
\text { (feet) }\end{array}$ \\
\hline \multicolumn{8}{|c|}{ Poinsett County-Continued } \\
\hline 12N05E16ABA1 & 354039 & 903333 & $4 / 3 / 2002$ & 10 & $4 / 17 / 2006$ & 12 & -2.0 \\
\hline 12N05E34ABA1 & 353805 & 903230 & $3 / 26 / 2002$ & 9.5 & $3 / 28 / 2006$ & 10.65 & -1.2 \\
\hline 12N07E04BAA1 & 354202 & 902060 & $3 / 26 / 2002$ & 4.24 & $3 / 29 / 2006$ & 6.93 & -2.7 \\
\hline \multicolumn{8}{|c|}{ Prairie County } \\
\hline 01N06W05CCB1 & 344353 & 914049 & $3 / 12 / 2002$ & 116.15 & $4 / 11 / 2006$ & 117.8 & -1.6 \\
\hline 01S04W28BDB1 & 343523 & 912630 & $3 / 12 / 2002$ & 83.17 & $4 / 11 / 2006$ & 97.28 & -14.1 \\
\hline 01S05W14BBC1 & 343722 & 913109 & $3 / 12 / 2002$ & 108.14 & $4 / 11 / 2006$ & 108.15 & 0.0 \\
\hline 01S05W31DDA1 & 343417 & 913432 & $3 / 12 / 2002$ & 95.34 & $4 / 11 / 2006$ & 103.6 & -8.3 \\
\hline 02N04W02BCB1 & 344916 & 912419 & $3 / 11 / 2002$ & 20.65 & $4 / 11 / 2006$ & 20.27 & 0.4 \\
\hline 02N04W32CCB1 & 344436 & 912738 & $3 / 12 / 2002$ & 84.08 & $4 / 11 / 2006$ & 84.72 & -0.6 \\
\hline 02N05W06BAB 1 & 344958 & 913421 & $3 / 11 / 2002$ & 88.4 & $4 / 11 / 2006$ & 89.19 & -0.8 \\
\hline 02N05W13AAB1 & 344805 & 912854 & $3 / 12 / 2002$ & 76.2 & $4 / 11 / 2006$ & 81.74 & -5.5 \\
\hline 02N05W29DDB2 & 344545 & 913309 & $3 / 12 / 2002$ & 116.89 & $4 / 11 / 2006$ & 118.84 & -2.0 \\
\hline 02N06W17ABB1 & 344809 & 913959 & $3 / 12 / 2002$ & 122.18 & $4 / 11 / 2006$ & 124.26 & -2.1 \\
\hline 02S06W14BBB1 & 343213 & 913729 & $3 / 12 / 2002$ & 75.65 & $4 / 11 / 2006$ & 74.29 & 1.4 \\
\hline 03N04W03AAC1 & 345439 & 912424 & $3 / 11 / 2002$ & 25.92 & $4 / 11 / 2006$ & 28.3 & -2.4 \\
\hline 03N05W03BDD2 & 345444 & 913115 & $3 / 11 / 2002$ & 64.12 & $4 / 11 / 2006$ & 67.7 & -3.6 \\
\hline 03N06W01BCB1 & 345455 & 913601 & $3 / 11 / 2002$ & 77.39 & $4 / 11 / 2006$ & 79.08 & -1.7 \\
\hline 03N06W19BDD1 & 345207 & 914110 & $3 / 11 / 2002$ & 83.73 & $4 / 12 / 2006$ & 86.73 & -3.0 \\
\hline 04N04W07ADC1 & 345850 & 912733 & $3 / 11 / 2002$ & 24.52 & $4 / 11 / 2006$ & 26.73 & -2.2 \\
\hline 04N05W07CDC1 & 345043 & 913441 & $3 / 11 / 2002$ & 74.33 & $4 / 12 / 2006$ & 76.54 & -2.2 \\
\hline 04N06W05CCC1 & 345934 & 914018 & $3 / 11 / 2002$ & 61.02 & $4 / 11 / 2006$ & 78.35 & -17.3 \\
\hline 04N07W03DCB1 & 345942 & 914412 & $3 / 11 / 2002$ & 85.93 & $4 / 12 / 2006$ & 87.33 & -1.4 \\
\hline 04N07W28BBA1 & 345701 & 914545 & $3 / 11 / 2002$ & 93.08 & $4 / 12 / 2006$ & 95.77 & -2.7 \\
\hline 05N05W14DCD1 & 350252 & 913034 & $3 / 11 / 2002$ & 36 & $4 / 12 / 2006$ & 39.62 & -3.6 \\
\hline \multicolumn{8}{|c|}{ Pulaski County } \\
\hline 01S10W29CC1 & 343538 & 920708 & $3 / 5 / 2002$ & 17.13 & $4 / 03 / 2006$ & 18.02 & -0.9 \\
\hline 02S10W14DC1 & 343205 & 920334 & $3 / 5 / 2002$ & 25.97 & $4 / 03 / 2006$ & 24.9 & 1.1 \\
\hline 02S10W16CCA1 & 343217 & 920549 & $3 / 5 / 2002$ & 21.98 & $4 / 03 / 2006$ & 27.27 & -5.3 \\
\hline \multicolumn{8}{|c|}{ Randolph County } \\
\hline 18N01E28AAD1 & 361040 & 905820 & $4 / 18 / 2002$ & 15.7 & $4 / 24 / 2006$ & 22 & -6.3 \\
\hline 18N01E34AAC1 & 360943 & 905729 & $3 / 28 / 2002$ & 16.63 & $4 / 17 / 2006$ & 18.18 & -1.6 \\
\hline 18N02E03DAD1 & 361336 & 905043 & $4 / 18 / 2002$ & 32.4 & $4 / 24 / 2006$ & 56 & -23.6 \\
\hline 18N02E17CBB1 & 361204 & 905356 & $4 / 18 / 2002$ & 16.1 & $4 / 24 / 2006$ & 23 & -6.9 \\
\hline 18N02E20BDA1 & 361125 & 905332 & $4 / 18 / 2002$ & 32.5 & $4 / 24 / 2006$ & 42.5 & -10.0 \\
\hline
\end{tabular}


Appendix 2. Information pertaining to the difference in water levels measured in 2002 and 2006 in the Mississippi River Valley alluvial aquifer in eastern Arkansas. - Continued

[Horizontal coordinate information is referenced to the North American Datum of 1983 (NAD83); *, control wells for duplicate measurements by USGS and NRCS]

\begin{tabular}{|c|c|c|c|c|c|c|c|}
\hline Station name & $\begin{array}{c}\text { Latitude } \\
\text { (degrees, } \\
\text { minutes, } \\
\text { seconds) }\end{array}$ & $\begin{array}{c}\text { Longitude } \\
\text { (degrees, } \\
\text { minutes, } \\
\text { seconds) }\end{array}$ & $\begin{array}{c}2002 \text { water- } \\
\text { level date }\end{array}$ & $\begin{array}{c}2002 \text { depth } \\
\text { to water } \\
\text { (feet below } \\
\text { land-surface } \\
\text { datum) }\end{array}$ & $\begin{array}{l}2006 \text { water- } \\
\text { level date }\end{array}$ & $\begin{array}{c}2006 \text { depth } \\
\text { to water } \\
\text { (feet below } \\
\text { land-surface } \\
\text { datum) }\end{array}$ & $\begin{array}{c}\text { Water-level } \\
\text { difference } \\
2002 \text { to } 2006 \\
\text { (feet) }\end{array}$ \\
\hline \multicolumn{8}{|c|}{ Randolph County-Continued } \\
\hline 18N02E22DCD1 & 361046 & 905105 & $3 / 28 / 2002$ & 36.34 & $4 / 17 / 2006$ & 37.47 & -1.1 \\
\hline $18 \mathrm{~N} 02 \mathrm{E} 34 \mathrm{BCC} 1$ & 360933 & 905150 & $4 / 18 / 2002$ & 56 & $4 / 24 / 2006$ & 31 & 25.0 \\
\hline 19N02E09ABD1 & 361826 & 905157 & $4 / 17 / 2002$ & 1.4 & $4 / 25 / 2006$ & 18 & -16.6 \\
\hline 19N02E22DAB1 & 361622 & 905049 & $4 / 17 / 2002$ & 0.3 & $4 / 24 / 2006$ & 15.5 & -15.2 \\
\hline 20N02E01ADD1 & 362424 & 904811 & $3 / 28 / 2002$ & 9.44 & $4 / 17 / 2006$ & 13.93 & -4.5 \\
\hline 20N02E12BAA1 & 362352 & 904848 & $4 / 17 / 2002$ & 4.3 & $4 / 25 / 2006$ & 12 & -7.7 \\
\hline 20N02E14DAB1 & 362232 & 904930 & $4 / 17 / 2002$ & 8.9 & $4 / 25 / 2006$ & 14 & -5.1 \\
\hline 20N02E21CDD1 & 362117 & 905107 & $4 / 17 / 2002$ & 6.2 & $4 / 25 / 2006$ & 12 & -5.8 \\
\hline 20N03E28BA1 & 362114 & 904538 & $3 / 28 / 2002$ & 11.75 & $4 / 17 / 2006$ & 12.86 & -1.1 \\
\hline 20N03E33CCA1 & 361941 & 904552 & $4 / 17 / 2002$ & 21.7 & $4 / 24 / 2006$ & 26 & -4.3 \\
\hline \multicolumn{8}{|c|}{ St. Francis County } \\
\hline 04N01E13ADA1 & 345755 & 905638 & $3 / 21 / 2002$ & 56.87 & $3 / 22 / 2006$ & 60.34 & -3.5 \\
\hline 04N01W20BBB1 & 345716 & 910759 & $4 / 16 / 2002$ & 57 & $5 / 15 / 2006$ & 59 & -2.0 \\
\hline 04N01W25DBD1 & 345549 & 910303 & $4 / 16 / 2002$ & 68 & $5 / 15 / 2006$ & 76 & -8.0 \\
\hline 04N01W28CDD1 & 345535 & 910634 & 4/1/2002 & 68 & $3 / 22 / 2006$ & 71.42 & -3.4 \\
\hline 04N02E16ACD1 & 345733 & 905341 & $4 / 16 / 2002$ & 49 & $5 / 15 / 2006$ & 41 & 8.0 \\
\hline 04N02E19BBB1 & 345701 & 905633 & $4 / 1 / 2002$ & 56.08 & $3 / 22 / 2006$ & 60.16 & -4.1 \\
\hline 04N02E27AAA1 & 345604 & 905220 & $4 / 16 / 2002$ & 46 & $5 / 15 / 2006$ & 48 & -2.0 \\
\hline 04N03E21DAD1 & 345623 & 904655 & 4/1/2002 & 59.23 & $3 / 22 / 2006$ & 58.14 & 1.1 \\
\hline 04N04E15ABA1 & 345752 & 903948 & $4 / 18 / 2002$ & 32 & $5 / 15 / 2006$ & 34 & -2.0 \\
\hline 04N05E22BBB1 & 345651 & 903357 & $3 / 22 / 2002$ & 28.48 & $3 / 22 / 2006$ & 27.02 & 1.5 \\
\hline 05N01E06CDA1 & 350437 & 910218 & $4 / 16 / 2002$ & 67 & $5 / 15 / 2006$ & 71 & -4.0 \\
\hline 05N01E15BCB1 & 350303 & 905942 & $4 / 1 / 2002$ & 61.34 & $3 / 22 / 2006$ & 67.19 & -5.8 \\
\hline 05N01E27BBA1 & 350136 & 905929 & $3 / 21 / 2002$ & 63.63 & $3 / 22 / 2006$ & 66.93 & -3.3 \\
\hline 05N02E20ADC1 & 350157 & 905437 & 4/1/2002 & 53.57 & $3 / 22 / 2006$ & 54.79 & -1.2 \\
\hline 05N03E20AAA2 & 350214 & 904801 & $3 / 21 / 2002$ & 104.55 & $3 / 22 / 2006$ & 103.87 & 0.7 \\
\hline 05N05E19DCA1 & 350128 & 903630 & $3 / 22 / 2002$ & 34.23 & $3 / 22 / 2006$ & 32.77 & 1.5 \\
\hline 05N05E33BCC1 & 350004 & 903506 & $4 / 18 / 2002$ & 28 & $5 / 15 / 2006$ & 29 & -1.0 \\
\hline 05N06E34CAB1 & 350026 & 902657 & $3 / 22 / 2002$ & 28.05 & $3 / 22 / 2006$ & 27.69 & 0.4 \\
\hline 06N01E33ACA2 & 350552 & 905942 & $4 / 1 / 2002$ & 64.42 & $3 / 22 / 2006$ & 67.14 & -2.7 \\
\hline 06N02E13DCA1 & 350813 & 905003 & $4 / 1 / 2002$ & 73.11 & $3 / 22 / 2006$ & 73.85 & -0.7 \\
\hline 06N02E15BDD1 & 350842 & 905247 & $4 / 1 / 2002$ & 52.23 & $3 / 22 / 2006$ & 60.15 & -7.9 \\
\hline 06N02E16CCC1 & 350804 & 905403 & $4 / 16 / 2002$ & 63 & $5 / 15 / 2006$ & 66.5 & -3.5 \\
\hline 06N02E24AAA1 & 350755 & 905002 & $4 / 1 / 2002$ & 70.26 & $3 / 22 / 2006$ & 71.46 & -1.2 \\
\hline 06N05E22ACC1 & 350723 & 903252 & $4 / 1 / 2002$ & 46.7 & $3 / 22 / 2006$ & 40.63 & 6.1 \\
\hline
\end{tabular}


Appendix 2. Information pertaining to the difference in water levels measured in 2002 and 2006 in the Mississippi River Valley alluvial aquifer in eastern Arkansas.-Continued

[Horizontal coordinate information is referenced to the North American Datum of 1983 (NAD83); *, control wells for duplicate measurements by USGS and NRCS]

\begin{tabular}{|c|c|c|c|c|c|c|c|}
\hline Station name & $\begin{array}{c}\text { Latitude } \\
\text { (degrees, } \\
\text { minutes, } \\
\text { seconds) }\end{array}$ & $\begin{array}{c}\text { Longitude } \\
\text { (degrees, } \\
\text { minutes, } \\
\text { seconds) }\end{array}$ & $\begin{array}{c}2002 \text { water- } \\
\text { level date }\end{array}$ & $\begin{array}{c}2002 \text { depth } \\
\text { to water } \\
\text { (feet below } \\
\text { land-surface } \\
\text { datum) }\end{array}$ & $\begin{array}{c}2006 \text { water- } \\
\text { level date }\end{array}$ & $\begin{array}{c}2006 \text { depth } \\
\text { to water } \\
\text { (feet below } \\
\text { land-surface } \\
\text { datum) }\end{array}$ & $\begin{array}{c}\text { Water-level } \\
\text { difference } \\
2002 \text { to } 2006 \\
\text { (feet) }\end{array}$ \\
\hline \multicolumn{8}{|c|}{ St. Francis County-Continued } \\
\hline 06N06E20ABB2 & 350747 & 902841 & $3 / 21 / 2002$ & 34 & $3 / 22 / 2006$ & 36.75 & -2.8 \\
\hline \multicolumn{8}{|c|}{ White County } \\
\hline 05N07W09AAA1 & 350447 & 914441 & $4 / 1 / 2002$ & 19.1 & $3 / 23 / 2006$ & 14.32 & 4.8 \\
\hline 05N07W10CCC1 & 350400 & 914436 & $4 / 1 / 2002$ & 7.68 & $3 / 23 / 2006$ & 9.01 & -1.3 \\
\hline 06N06W04BAA1 & 351047 & 913910 & $4 / 1 / 2002$ & 37.97 & $3 / 23 / 2006$ & 35.47 & 2.5 \\
\hline 06N06W04BAD1 & 351037 & 913903 & $4 / 16 / 2002$ & 41 & $4 / 25 / 2006$ & 40.8 & 0.2 \\
\hline 06N06W13DBB1 & 350918 & 913552 & $4 / 16 / 2002$ & 48.5 & $4 / 25 / 2006$ & 47.3 & 1.2 \\
\hline 06N06W18BBC1 & 350851 & 914152 & $4 / 1 / 2002$ & 19 & $3 / 23 / 2006$ & 18.13 & 0.9 \\
\hline 06N06W18BCA1 & 350835 & 914150 & $4 / 16 / 2002$ & 21.5 & $4 / 25 / 2006$ & 20.3 & 1.2 \\
\hline 06N06W34AAB1 & 350624 & 913754 & $4 / 1 / 2002$ & 59.86 & $3 / 23 / 2006$ & 60.63 & -0.8 \\
\hline 06N07W17DCC1 & 350822 & 914635 & $4 / 1 / 2002$ & 13.94 & $3 / 23 / 2006$ & 15.7 & -1.8 \\
\hline 06N08W13ABA1 & 350908 & 914824 & $4 / 1 / 2002$ & 8.2 & $3 / 23 / 2006$ & 13.6 & -5.4 \\
\hline 06N08W26DDB1 & 350640 & 914931 & $4 / 1 / 2002$ & 12.62 & $3 / 23 / 2006$ & 14.47 & -1.9 \\
\hline 07N05W01AAA1 & 351553 & 912858 & $3 / 29 / 2002$ & 15 & $3 / 23 / 2006$ & 17.52 & -2.5 \\
\hline 07N05W32BAB 1 & 351137 & 913406 & $4 / 1 / 2002$ & 27.76 & $3 / 23 / 2006$ & 32.64 & -4.9 \\
\hline 08N04W06CCB1 & 352028 & 912847 & $3 / 29 / 2002$ & 16.02 & $3 / 23 / 2006$ & 20.5 & -4.5 \\
\hline 08N05W32CBC1 & 351616 & 913417 & $3 / 29 / 2002$ & 0.6 & $3 / 23 / 2006$ & 4.17 & -3.6 \\
\hline \multicolumn{8}{|c|}{ Woodruff County } \\
\hline 04N03W03AB1 & 350021 & 911820 & $3 / 20 / 2002$ & 12.13 & $3 / 28 / 2006$ & 14.61 & -2.5 \\
\hline 05N01W13CDC1 & 350244 & 910331 & $3 / 25 / 2002$ & 71.6 & $4 / 05 / 2006$ & 74.6 & -3.0 \\
\hline 05N01W31CCC1 & 350106 & 910900 & $3 / 25 / 2002$ & 57.3 & $4 / 05 / 2006$ & 59.1 & -1.8 \\
\hline 05N02W20DCB 1 & 350208 & 911356 & $3 / 20 / 2002$ & 13.17 & $3 / 28 / 2006$ & 15.25 & -2.1 \\
\hline 05N03W25DDB1 & 350133 & 911531 & $3 / 25 / 2002$ & 12.7 & $4 / 05 / 2006$ & 12.7 & 0.0 \\
\hline 05N04W12DBA1 & 350427 & 912211 & $3 / 20 / 2002$ & 3.11 & $3 / 27 / 2006$ & 6.16 & -3.1 \\
\hline 06N01W06BAB1 & 351048 & 910835 & $3 / 20 / 2002$ & 32.8 & $3 / 28 / 2006$ & 34.53 & -1.7 \\
\hline 06N02W19AAA1 & 350802 & 911419 & $3 / 25 / 2002$ & 46.5 & $4 / 05 / 2006$ & 45.5 & 1.0 \\
\hline 06N03W15BAB1 & 350903 & 911807 & $3 / 20 / 2002$ & 4.38 & $3 / 27 / 2006$ & 6.63 & -2.3 \\
\hline 06N03W31BCB1 & 350623 & 912144 & $3 / 20 / 2002$ & 0.98 & $3 / 27 / 2006$ & 2.86 & -1.9 \\
\hline 07N01W04ACB1 & 351541 & 910626 & $3 / 26 / 2002$ & 60 & $4 / 05 / 2006$ & 60.9 & -0.9 \\
\hline 07N03W06BAC1 & 351607 & 912109 & $3 / 27 / 2002$ & 25.15 & $4 / 05 / 2006$ & 24.4 & 0.8 \\
\hline 07N03W19AAA1 & 351335 & 912025 & $3 / 20 / 2002$ & 11.75 & $3 / 27 / 2006$ & 12.95 & -1.2 \\
\hline 07N03W31BBA1 & 351152 & 912103 & $3 / 27 / 2002$ & 9.4 & $4 / 05 / 2006$ & 11.9 & -2.5 \\
\hline 08N01W06DDD1 & 352028 & 910747 & $3 / 20 / 2002$ & 41.9 & $3 / 28 / 2006$ & 44.57 & -2.7 \\
\hline 08N02W27DDB1 & 351711 & 911107 & $3 / 26 / 2002$ & 27 & $4 / 05 / 2006$ & 27 & 0.0 \\
\hline 08N02W31DDD1 & 351611 & 911411 & $3 / 20 / 2002$ & 2.51 & $3 / 28 / 2006$ & 4.02 & -1.5 \\
\hline
\end{tabular}


Appendix 2. Information pertaining to the difference in water levels measured in 2002 and 2006 in the Mississippi River Valley alluvial aquifer in eastern Arkansas.-Continued

[Horizontal coordinate information is referenced to the North American Datum of 1983 (NAD83); *, control wells for duplicate measurements by USGS and NRCS]

\begin{tabular}{|c|c|c|c|c|c|c|c|}
\hline Station name & $\begin{array}{c}\text { Latitude } \\
\text { (degrees, } \\
\text { minutes, } \\
\text { seconds) }\end{array}$ & $\begin{array}{l}\text { Longitude } \\
\text { (degrees, } \\
\text { minutes, } \\
\text { seconds) }\end{array}$ & $\begin{array}{l}2002 \text { water- } \\
\text { level date }\end{array}$ & $\begin{array}{l}2002 \text { depth } \\
\text { to water } \\
\text { (feet below } \\
\text { land-surface } \\
\text { datum) }\end{array}$ & $\begin{array}{c}2006 \text { water- } \\
\text { level date }\end{array}$ & $\begin{array}{l}2006 \text { depth } \\
\text { to water } \\
\text { (feet below } \\
\text { land-surface } \\
\text { datum) }\end{array}$ & $\begin{array}{c}\text { Water-level } \\
\text { difference } \\
2002 \text { to } 2006 \\
\text { (feet) }\end{array}$ \\
\hline \multicolumn{8}{|c|}{ Woodruff County_Continued } \\
\hline 08N03W31AAD1 & 351655 & 912028 & $3 / 20 / 2002$ & 24.29 & $3 / 27 / 2006$ & 21.95 & 2.3 \\
\hline 08N04W27AAA1 & 351757 & 912341 & $3 / 20 / 2002$ & 3.21 & $3 / 27 / 2006$ & 13.1 & -9.9 \\
\hline 09N03W28ABB1 & 352310 & 911845 & $3 / 26 / 2002$ & 19.9 & $4 / 05 / 2006$ & 19.6 & 0.3 \\
\hline 09N03W29AAD1 & 352258 & 911921 & $3 / 20 / 2002$ & 21.42 & $3 / 27 / 2006$ & 22.53 & -1.1 \\
\hline 09N03W32ACA1 & 352205 & 911936 & $3 / 26 / 2002$ & 19 & $4 / 05 / 2006$ & 21.6 & -2.6 \\
\hline
\end{tabular}


Appendix 3. Specific conductance and temperature data collected from wells completed in the Mississippi River Valley alluvial aquifer in eastern Arkansas, summer 2006.

[Horizontal coordinate information is referenced to the North American Datum of 1983 (NAD83); uS/cm, microsiemens per centimeter at 25 degrees Celsius]

\begin{tabular}{|c|c|c|c|c|c|}
\hline Station name & $\begin{array}{c}\text { Latitude } \\
\text { (degrees, } \\
\text { minutes, } \\
\text { seconds) }\end{array}$ & $\begin{array}{c}\text { Longitude } \\
\text { (degrees, } \\
\text { minutes, } \\
\text { seconds) }\end{array}$ & Date & 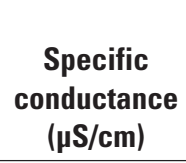 & $\begin{array}{c}\text { Temperature } \\
\text { (degrees } \\
\text { Celsius) }\end{array}$ \\
\hline \multicolumn{6}{|c|}{ Arkansas County } \\
\hline 02S04W14CD1 & 343100 & 912445 & $6 / 21 / 2006$ & 887 & 19.5 \\
\hline 04S03W17ADD1 & 342102 & 912058 & $6 / 21 / 2006$ & 1,080 & 19.5 \\
\hline 04S06W16BD1 & 342130 & 914000 & $6 / 21 / 2006$ & 720 & 20.0 \\
\hline 05S04W07CCC1 & 341555 & 912932 & $6 / 21 / 2006$ & 1,010 & 19.6 \\
\hline \multicolumn{6}{|c|}{ Ashley County } \\
\hline 15S04W23DBD1 & 332247 & 912852 & $6 / 19 / 2006$ & 612 & 19.2 \\
\hline 17S07W05CDD1 & 331502 & 915050 & $6 / 19 / 2006$ & 689 & 19.7 \\
\hline 18S08W01AAB1 & 331015 & 915225 & $6 / 19 / 2006$ & 583 & 19.8 \\
\hline \multicolumn{6}{|c|}{ Chicot County } \\
\hline 13S03W35BAC1 & 333154 & 912246 & $6 / 20 / 2006$ & 376 & 23.6 \\
\hline 17S01W06BCC1 & 331501 & 911505 & $6 / 20 / 2006$ & 797 & 19.2 \\
\hline 17S03W09ADA1 & 331415 & 912426 & $6 / 20 / 2006$ & 2,960 & 20.3 \\
\hline \multicolumn{6}{|c|}{ Clay County } \\
\hline 19N08E02ABB1 & 361859 & 901104 & $6 / 28 / 2006$ & 371 & 16.8 \\
\hline 19N08E28BB1 & 361519 & 901318 & $6 / 28 / 2006$ & 356 & 16.7 \\
\hline 20N08E24DDA1 & 362057 & 900934 & $6 / 28 / 2006$ & 362 & 17.0 \\
\hline 21N04E34DDC1 & 362445 & 903729 & $6 / 28 / 2006$ & 267 & 17.9 \\
\hline \multicolumn{6}{|c|}{ Craighead County } \\
\hline 13N03E29AAA1 & 354403 & 904713 & $6 / 28 / 2006$ & 1,140 & 18.2 \\
\hline 15N06E19AAB1 & 355517 & 902857 & $6 / 27 / 2006$ & 513 & 19.2 \\
\hline 16N07E32ADD1 & 355813 & 902138 & $6 / 27 / 2006$ & 407 & 18.9 \\
\hline \multicolumn{6}{|c|}{ Crittenden County } \\
\hline 06N07E13BAA1 & 350850 & 901808 & $6 / 27 / 2006$ & 516 & 18.5 \\
\hline 07N07E31CCC1 & 351042 & 902359 & $6 / 27 / 2006$ & 496 & 18.0 \\
\hline \multicolumn{6}{|c|}{ Cross County } \\
\hline 07N01E05CDA1 & 351518 & 910049 & $6 / 27 / 2006$ & 961 & 19.0 \\
\hline 09N01E33BBA1 & 352204 & 905959 & $6 / 27 / 2006$ & 566 & 18.7 \\
\hline 09N05E32BDB1 & 352151 & 903512 & $6 / 27 / 2006$ & 569 & 18.5 \\
\hline \multicolumn{6}{|c|}{ Desha County } \\
\hline 09S04W06BCA1 & 335756 & 913243 & $6 / 20 / 2006$ & 877 & 20.6 \\
\hline 10S03W26CAA1 & 334806 & 912145 & $6 / 20 / 2006$ & 826 & 19.4 \\
\hline \multicolumn{6}{|c|}{ Drew County } \\
\hline 11S04W08DBA1 & 334532 & 913136 & $6 / 19 / 2006$ & 380 & 21.1 \\
\hline 14S04W27AA1 & 332734 & 912925 & $6 / 19 / 2006$ & 637 & 19.8 \\
\hline \multicolumn{6}{|c|}{ Greene County } \\
\hline 16N06E28ABB1 & 355938 & 902657 & $6 / 28 / 2006$ & 819 & 17.8 \\
\hline
\end{tabular}


Appendix 3. Specific conductance and temperature data collected from wells completed in the Mississippi River Valley alluvial aquifer in eastern Arkansas, summer 2006.-Continued

[Horizontal coordinate information is referenced to the North American Datum of 1983 (NAD83); uS/cm, microsiemens per centimeter at 25 degrees Celsius]

\begin{tabular}{|c|c|c|c|c|c|}
\hline Station name & $\begin{array}{c}\text { Latitude } \\
\text { (degrees, } \\
\text { minutes, } \\
\text { seconds) }\end{array}$ & $\begin{array}{c}\text { Longitude } \\
\text { (degrees, } \\
\text { minutes, } \\
\text { seconds) }\end{array}$ & Date & $\begin{array}{c}\text { Specific } \\
\text { conductance } \\
\text { ( } \mathrm{S} / \mathrm{cm})\end{array}$ & $\begin{array}{c}\text { Temperature } \\
\text { (degrees } \\
\text { Celsius) }\end{array}$ \\
\hline \multicolumn{6}{|c|}{ Jackson County } \\
\hline 09N02W32CBB1 & 352152 & 911348 & $6 / 28 / 2006$ & 426 & 18.4 \\
\hline 10N02W29ABB1 & 352829 & 911312 & $6 / 28 / 2006$ & 299 & 18.7 \\
\hline 14N01W09AAA1 & 355220 & 910515 & $6 / 28 / 2006$ & 460 & 18.1 \\
\hline \multicolumn{6}{|c|}{ Jefferson County } \\
\hline 03S09W18CC2 & 342656 & 920139 & $6 / 20 / 2006$ & 642 & 20.2 \\
\hline 03S09W31DDA1 & 342415 & 920049 & $6 / 20 / 2006$ & 608 & 24.6 \\
\hline 04S08W13DCB1 & 342123 & 914926 & $6 / 20 / 2006$ & 527 & 20.0 \\
\hline 06S06W23AAD1 & 341007 & 913712 & $6 / 20 / 2006$ & 675 & 19.7 \\
\hline \multicolumn{6}{|c|}{ Lawrence County } \\
\hline 16N02E05BA1 & 360326 & 905352 & $6 / 28 / 2006$ & 599 & 18.1 \\
\hline \multicolumn{6}{|c|}{ Lee County } \\
\hline 01N03E23CCC1 & 344025 & 904604 & $6 / 22 / 2006$ & 692 & 19.2 \\
\hline 03N03E32CAB1 & 344933 & 904926 & $6 / 22 / 2006$ & 540 & 18.8 \\
\hline \multicolumn{6}{|c|}{ Lincoln County } \\
\hline 08S04W19CC1 & 340021 & 913205 & $6 / 19 / 2006$ & 843 & 19.4 \\
\hline 09S06W04BCD1 & 335821 & 914346 & $6 / 19 / 2006$ & 372 & 19.1 \\
\hline 09S07W01DC1 & 335714 & 914637 & $6 / 19 / 2006$ & 548 & 19.9 \\
\hline \multicolumn{6}{|c|}{ Lonoke County } \\
\hline 01N07W29BBB1 & 344114 & 914720 & $6 / 21 / 2006$ & 458 & 20.7 \\
\hline 02N07W02BBA1 & 344957 & 914338 & $6 / 21 / 2006$ & 415 & 20.9 \\
\hline 02S08W13BBB1 & 343232 & 914935 & $6 / 21 / 2006$ & 712 & 20.0 \\
\hline \multicolumn{6}{|c|}{ Mississippi County } \\
\hline 12N08E20DAD1 & 353842 & 901458 & $6 / 27 / 2006$ & 411 & 19.4 \\
\hline \multicolumn{6}{|c|}{ Monroe County } \\
\hline 01N02W12CBC1 & 344242 & 911032 & $6 / 22 / 2006$ & 985 & 19.2 \\
\hline 01N04W33BB2 & 343958 & 912646 & $6 / 22 / 2006$ & 743 & 19.4 \\
\hline 01S04W01BAB1 & 343906 & 912317 & $6 / 22 / 2006$ & 712 & 19.4 \\
\hline 03N03W36AAA1 & 345027 & 911547 & $6 / 22 / 2006$ & 806 & 18.9 \\
\hline \multicolumn{6}{|c|}{ Phillips County } \\
\hline 02S01E28CCB1 & 342916 & 910058 & $6 / 22 / 2006$ & 606 & 19.5 \\
\hline \multicolumn{6}{|c|}{ Poinsett County } \\
\hline 10N03E14DAB1 & 352947 & 904405 & $6 / 27 / 2006$ & 558 & 19.9 \\
\hline 10N03E35CDD1 & 352656 & 904436 & $6 / 27 / 2006$ & 532 & 19.4 \\
\hline 11N02E26AAB1 & 353350 & 905034 & $6 / 27 / 2006$ & 787 & 19.2 \\
\hline 11N07E18CAB1 & 353435 & 902320 & $6 / 27 / 2006$ & 537 & 19.1 \\
\hline
\end{tabular}


Appendix 3. Specific conductance and temperature data collected from wells completed in the Mississippi River Valley alluvial aquifer in eastern Arkansas, summer 2006.-Continued

[Horizontal coordinate information is referenced to the North American Datum of 1983 (NAD83); uS/cm, microsiemens per centimeter at 25 degrees Celsius]

\begin{tabular}{|c|c|c|c|c|c|}
\hline Station name & $\begin{array}{c}\text { Latitude } \\
\text { (degrees, } \\
\text { minutes, } \\
\text { seconds) }\end{array}$ & $\begin{array}{c}\text { Longitude } \\
\text { (degrees, } \\
\text { minutes, } \\
\text { seconds) }\end{array}$ & Date & $\begin{array}{c}\text { Specific } \\
\text { conductance } \\
(\mu \mathrm{S} / \mathrm{cm})\end{array}$ & $\begin{array}{c}\text { Temperature } \\
\text { (degrees } \\
\text { Celsius) }\end{array}$ \\
\hline \multicolumn{6}{|c|}{ Prairie County } \\
\hline 01S04W28BD1 & 343521 & 912624 & $6 / 22 / 2006$ & 952 & 19.6 \\
\hline 02N05W06BAB1 & 344958 & 913421 & $6 / 22 / 2006$ & 1,000 & 19.7 \\
\hline 02N05W29DDB2 & 344545 & 913309 & $6 / 22 / 2006$ & 886 & 19.1 \\
\hline \multicolumn{6}{|c|}{ Pulaski County } \\
\hline 02S10W14DC1 & 343205 & 920334 & $6 / 21 / 2006$ & 830 & 20.3 \\
\hline \multicolumn{6}{|c|}{ Randolph County } \\
\hline 18N01E34AAC1 & 360943 & 905729 & $6 / 28 / 2006$ & 761 & 17.7 \\
\hline \multicolumn{6}{|c|}{ St. Francis County } \\
\hline 04N01E13DDA1 & 345708 & 905638 & $6 / 22 / 2006$ & 714 & 19.5 \\
\hline 04N01W24DA1 & 345649 & 910247 & $6 / 22 / 2006$ & 892 & 19.5 \\
\hline 06N02E13DCA1 & 350813 & 905003 & $6 / 23 / 2006$ & 755 & 19.1 \\
\hline \multicolumn{6}{|c|}{ White County } \\
\hline 06N06W34AAB1 & 350624 & 913754 & $6 / 23 / 2006$ & 843 & 18.0 \\
\hline \multicolumn{6}{|c|}{ Woodruff County } \\
\hline 06N01W10AB1 & 350945 & 910513 & $6 / 23 / 2006$ & 925 & 19.1 \\
\hline 07N01W32CCD1 & 351046 & 910741 & $6 / 23 / 2006$ & 589 & 18.3 \\
\hline 08N03W31AAD1 & 351655 & 912028 & $6 / 23 / 2006$ & 668 & 18.5 \\
\hline
\end{tabular}


Publishing support provided by:

Lafayette and Rolla Publishing Service Centers

For more information concerning the research described in the report:

U.S. Geological Survey

Arkansas Water Science Center

401 Hardin Road

Little Rock, AR 72211-3528

(501) 228-3600

http://ar.water.usgs.gov 


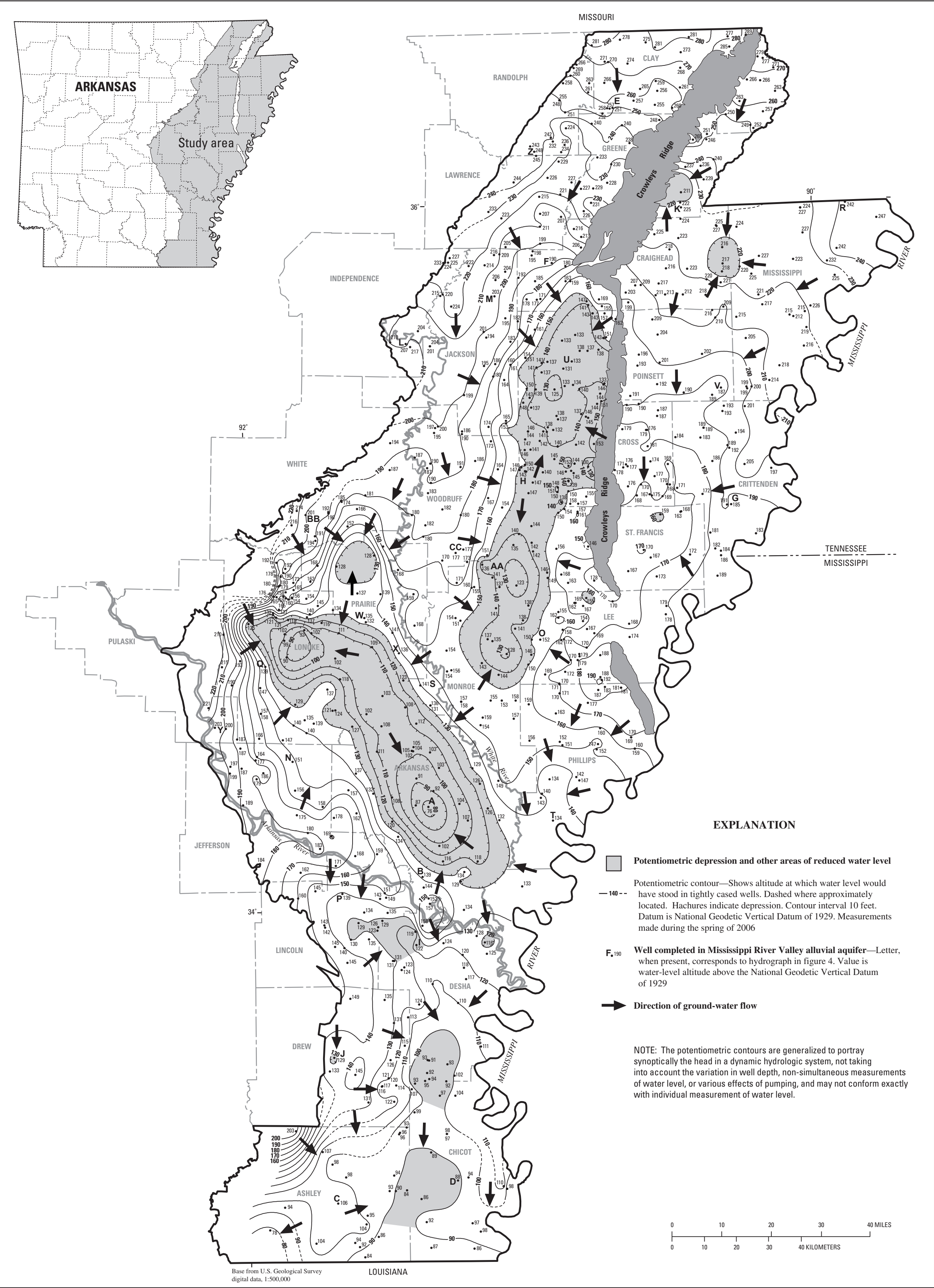

Potentiometric Surface of the Mississippi River Valley Alluvial Aquifer, Spring 2006 

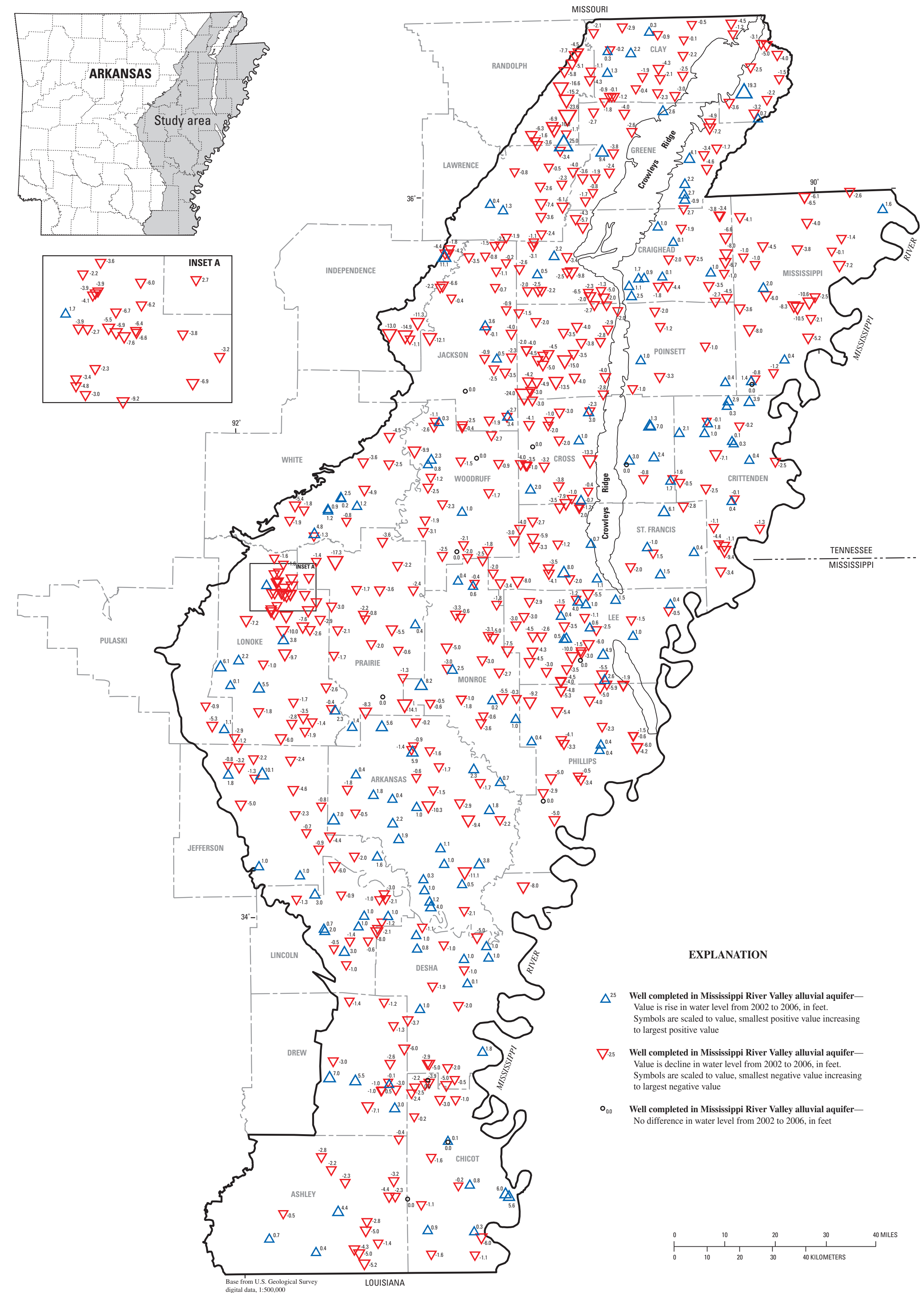

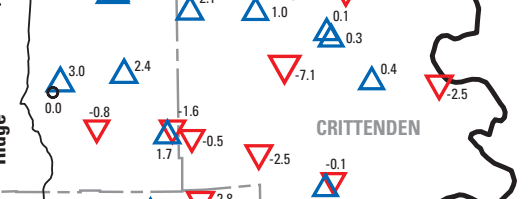

Difference in Water Level from 2002 to 2006 in the Mississippi River Valley Alluvial Aquifer in Eastern Arkansas 
